\title{
Multisensory processing in full-body illusions
}

Citation for published version (APA):

Maselli, A. (2019). Multisensory processing in full-body illusions. [Doctoral Thesis, Maastricht University]. Maastricht University. https://doi.org/10.26481/dis.20190501am

Document status and date:

Published: 01/01/2019

DOI:

10.26481/dis.20190501am

Document Version:

Publisher's PDF, also known as Version of record

\section{Please check the document version of this publication:}

- A submitted manuscript is the version of the article upon submission and before peer-review. There can be important differences between the submitted version and the official published version of record.

People interested in the research are advised to contact the author for the final version of the publication, or visit the DOI to the publisher's website.

- The final author version and the galley proof are versions of the publication after peer review.

- The final published version features the final layout of the paper including the volume, issue and page numbers.

Link to publication

\footnotetext{
General rights rights.

- You may freely distribute the URL identifying the publication in the public portal. please follow below link for the End User Agreement:

www.umlib.nl/taverne-license

Take down policy

If you believe that this document breaches copyright please contact us at:

repository@maastrichtuniversity.nl

providing details and we will investigate your claim.
}

Copyright and moral rights for the publications made accessible in the public portal are retained by the authors and/or other copyright owners and it is a condition of accessing publications that users recognise and abide by the legal requirements associated with these

- Users may download and print one copy of any publication from the public portal for the purpose of private study or research.

- You may not further distribute the material or use it for any profit-making activity or commercial gain

If the publication is distributed under the terms of Article $25 \mathrm{fa}$ of the Dutch Copyright Act, indicated by the "Taverne" license above, 


\title{
MULTISENSORY PROCESSING IN
}

\author{
FULL-BODY ILLUSIONS
}





\section{MULTISENSORY PROCESSING IN}

\section{FULL-BODY ILLUSIONS}

\section{DISSERTATION}

to obtain the degree of Doctor at Maastricht University

Department of Cognitive Neuroscience

Faculty of Psychology and Neuroscience

Maastricht University

$1^{\text {st }}$ of May 2019 at 14:00 hours

by

Antonella Maselli 


\title{
SUPERVISORS:
}

Prof. Dr. Beatrice de Gelder

Prof. Dr. Mel Slater, Universitat de Barcelona

\author{
Assessment Committee: \\ Prof. Dr. Sonja Kotz, Chair \\ Prof. Dr. Jan van den Stock, KU Leuven \\ Dr.Aline de Borst, University College London \\ Dr. Maarten Vaessen
}

INSTITUTIONS AND/OR ORGANISATIONS THAT CONTRIBUTED FINANCIALLY TO THE CREATION OF THE THESIS:

European Commission through the ERC Project TRAVERSE (Contract 227985), awarded to Prof. Mel Slater 
A Ivano e Irene 



\section{Table of Content}

Chapter 1

Introduction

Chapter 2

The building blocks of the full body ownership illusion

[Maselli, A., and Slater, M., Front. Hum. Neurosci. 7, 83, 2013]

Chapter 3

The sense of body ownership relaxes temporal constraints for multisensory integration [Maselli,A., Kilteni, K., López-Moliner, J., and Slater, M., Sci. Rep. 6, 30628, 2016]

Chapter 4

The body fades away: investigating the effects of transparency of an embodied virtual body on pain threshold and body

[Martini, M., Kilteni, K., Maselli,A., and Sanchez-vives, M.V., Sci. Rep. 5, 13948, 2015]

ChAPTER 5

Over my fake body: body ownership illusions for studying the multisensory basis of own-body perception

[Kilteni*, K., Maselli*,A., Kording, K.P., and Slater, M. Front. Hum. Neurosci. 9:141, 2015]

* Equal contribution

CHAPTER 6

Sliding perspectives: dissociating ownership from self-location during full body illusions in virtual reality

[Maselli,A., and Slater, M. Front. Hum. Neurosci. 8:693, 2014]

CHAPTER 7

Allocentric and egocentric manipulations of the sense of self-location in full-body illusions and their relation with the sense of body ownership

[Maselli,A. , Cognitive Processes. 16, 2, 2015] 



\section{CHAPTER 1}

\section{INTRODUCTION}



Our own bodies are unique objects of perception. This uniqueness is rooted in two main aspects. First, the perception of our body pervades our all existence: sensations from our bodies are ubiquitous and omnipresent, as they cannot be switched off at will. Second, the perception of our own bodies exceeds in complexity other forms of perceptual experiences in what concerns the related multisensory dimension. In fact, beside the sensory channels typically available for exploring the external world, when it comes to building up a representation of our own bodies, we have access to additional information from proprioception, interoception and vestibular sensations.

The ubiquity and complexity that characterize the own body as an object of perception, when considered one beside the other, pose an interesting conundrum. The ubiquitous perception of our own bodies needs to be extremely effortless and almost transparent to our consciousness so to not interfere with our daily activities of whatever sort. At the same time, it needs to be informative enough so to allow us to optimally explore and act within the environment. The non-trivial question arising is therefore "How comes that the massive sensory processing involved in creating a stable and down-to-earth representation of our bodies does happen so effortlessly that we are only occasionally conscious about it?". The answer to this question is far from being obvious and in the last decades a large community of philosophers, neurologists and cognitive neuroscientists devoted their work to shed some light on this problem. This collective effort has highlighted the multisensory and dynamic nature of the body percept, and established evidences for its neural correlates to be spread in a network of cortical and subcortical brain areas. The work presented in this thesis aimed at providing a contribution to this collective effort.

Bringing own body perception under scientific scrutiny is arduous. As a form of self-perception, it can be inspected either through descriptions of subjective experiences, or indirectly through behavioural and/or neurophysiological assessments, which should be nevertheless correlated to some extent with subjective accounts. In addition, the classical experimental approaches adopted in multisensory integration research are not easily applicable in the context of own body perception because the temporal and spatial dissociation of bodily signals is not trivial to achieve. In fact, the visual, somatosensory, motor and haptic sensations related to whatever bodily experience are locked in an intrinsic spatiotemporal "synchrony" dictated by physical laws. These difficulties have confined for long time the study of the multisensory mechanisms involved in own body perception to the domain of neuropsychology, in which the study of conditions characterized by impaired self-body perception (for example, the misattribution of one's own arm to someone else), provided the first important insights. Only recently, the scientific inquire about the multisensory basis of self-perception could be extended to the normotypical population thanks to the introductions of novel experimental approaches, mainly relying on bodily illusions and prompted by new technological advances such as immersive virtual reality devices (de Gelder et al., 2018; Sanchez-Vives and Slater, 2005).

Various experimental paradigms have been established in the last decades that can alter the perception of one's own body in a predictable and systematic manner. These paradigms are based 
on the manipulation of sensory stimuli so that, by delivering conflicting information about the body, illusory experiences arise as the brain "looks for" an explanatory solution to the conflict. In this way, it is possible to let people experience their noses elongating, or experience a fake rubber as one's own to the point of engaging in gross defensive reactions when the fake hand is threatened. The Pinocchio and the Rubber Hand Illusions are just two examples from a battery of body illusions that, because of their reproducibility and the concurrent possibility to monitor their behavioural and physiological correlates in controlled experimental environment, have been proven powerful tools for the investigation of the neural and cognitive mechanisms underlying self-body perception and corporeal awareness.

The works presented in this dissertation capitalize on the established use of bodily illusions as research tools, with the aim of advancing our understanding of the multisensory mechanisms underlying the perception of our bodies. Making use of Immersive Virtual Reality (IVR) technology, four experimental studies have been carried out to tackle issues related with the multisensory processing underlying the sense of body ownership, namely the feeling that the our body and its parts belong to the self, and the sense of self-location, i.e., the feeling of where the self is located in space. More specifically, the first study was tailored for identifying the "building blocks" of the sense of body ownership in terms of the major multisensory components involved and of their interaction. Two other studies focused on the impact of illusory ownership experience in the subsequent processing of sensory stimuli, i.e. visuotactile integration and pain. The fourth experimental study aimed at disentangling the multisensory mechanisms that can selectively affect body-ownership and self-location. In the other two review studies presented in this dissertation, results from the experiments performed are placed and discussed in the context of the relevant literature. Concurrently, proposals for common frameworks have been advanced that could account for the plethora of results, sometimes controversial, presented and discussed in the literature. 


\section{BACKGROUD}

\subsection{Altered body perceptions of neurological origin}

Bizarre conditions in which people experience abnormal perceptions of their own bodies have been the first inquiring tool for scientifically approaching the problem of how the information conveyed into the brain through different sensory channels is merged and integrated into the own-body percept. Because of their recurrence, phantom limbs have been the most documented and studied of these phenomena.Virtually all amputees report having phantom limbs experiences, which consist in vivid sensations that closely resemble somatosensory perceptions of the removed real limb, and that in some case are extremely painful (Ramachandran and Hirstein, 1998). Based on the vast phenomenology of phantom limbs sensations, it was early suggested that explanations for these phenomena must be sought in the "same brain processes that underlie the experience of the body when it is intact" (Melzack, 1990). Melzack further proposed that these brain processes should occur in a neural network encompassing several brain areas (the "neuromatrix"), which is at first genetically wired and later shaped through experience. In his proposal, Melzack also advanced the idea that "the feeling that all the parts of the body are uniquely one's own" - a.k.a. the sense of body ownership - is generated by activity in the neuromatrix, as all the perceptual experiences "that are felt to originate from the body".

Several other neurological disorders that involve the impairment of different aspects of own-body perception have contributed in gathering knowledge about the complex brain processes underlying own-body perception (Berlucchi and Aglioti, 1997; Head and Holmes, 1911). Particularly relevant for the work presented in this dissertation, are the cases of disturbed sensations of body ownership (DSO), in which patients do not recognize one of their limbs as part of their body, and the case of out-of-body experiences (OBEs), involving the feeling of floating outside one's own body and seeing it from an elevated perspective. The contribution that these conditions have given to the current understanding of own-body perception is briefly reviewed in the following sections.

\subsubsection{Disturbed Sensations of limb OWnership (DSO)}

Neurological conditions characterized by abnormal experiences of the body or of a body part, are typically associated with damage in a rich set of cortical and subcortical structures in the right hemisphere (Berlucchi and Aglioti, 1997, 2010). Among numerous interesting conditions, a prime example is the cases of asomatognosia and somatoparaphrenia (Feinberg, 2010). Asomatognosia literally means "lack of knowledge of the body". It was first described as a condition in which patients are not aware of their disease "in parts or side of the body, with forgetting or not recognition of their possession" (Gerstmann, 1942). In somatoparaphrenia, these symptoms are associated with some forms of delusion and confabulation, often consisting in the misattribution of one of the limb contralateral to the lesion to someone else (Critchley, 1955).

Group studies on patients affected by these "disturbed sensations of limb ownership" (DSO) (Baier and Karnath, 2008) have revealed consistent damages in the temporoparietal cortex, the posterior insula, and in several subcortical areas (Feinberg et al., 2010; Gandola et al., 2012; Romano et 
al., 2014). Interestingly, a consistent reduction in the insula cortical volume was also shown in individuals suffering from xenomelia, the obsessing desire of amputating one's own limb (Hilti et al., 2013). Instead, lesions in the right frontal cortex have been consistently observed only in somatoparaphrenic patients (Feinberg and Roane, 2005; Gandola et al., 2012; Invernizzi et al., 2013). Crucially, these DSO syndromes are rare and most often associated with a number of other conditions, from hemiplegia to spatial neglect (Gerstmann 1942;Vallar \& Ronchi 2009), which makes it practically unfeasible to pin-point specific areas that are potentially responsible for shaping the experience of the body as part of the self. Despite this, the fact that the implicated brain areas are involved with multisensory integration and high-order cognitive processes provided the first evidence for the tight link between the sense of body ownership and multisensory integration (Bisiach et al., 1991; Vallar and Ronchi, 2009). Furthermore, this link pushed forward the idea that the sense of body ownership emerges as the result of distributed activity processing of massive amount of information in an extensive fronto-temporo-parietal network (Feinberg and Venneri, 2014; Vallar and Ronchi, 2009).

\subsubsection{OUT-OF-BODY EXPERIENCES (OBE)}

OBEs are another type of altered body perception that provided interesting insights into the brain processes underlying corporeal awareness. OBEs consist in transitory episodes in which the self, intended as "the centre of awareness", is experienced as dislocated from the physical body, which is typically seen from an elevated perspective (Blackmore, 1982; Brugger et al., 1997). OBEs are therefore associated with abnormal perception of self-location, in which the spatial unity between the self and the body is broken.

These illusory experiences have been associated with neurological conditions, such as migraine (Lippman, 1953), traumatic and vascular brain damage (Todd and Dewhurst, 1955), focal epilepsy (Devinsky et al.,1989) and, more recently, to dizziness (Lopez and Elzière, 2017). The first neurological accounts of OBEs reported about associated brain damages mainly in the temporal, parietal and occipital areas, with a predominance of the right hemisphere (see Blanke \& Mohr 2005 for a review). Later group lesion studies showed a consistent implication of the right temporo-parietal junction, with particular implication of the anterior angular gyrus and the posterior part of the superior temporal gyrus (Blanke and Arzy, 2005). It was further shown that focal electrical stimulation of the temporo-parietal junction (TPJ) could systematically trigger OBEs (Blanke et al., 2002). Interestingly, a positron-emission tomographic (PET) scanning recorded during experiences of disembodiment, without autoscopy, showed specific brain activation in the TPJ and interconnected subcortical regions (De Ridder et al., 2007).

Because of the associated sensation of flying and floating, most of the early authors attributed OBEs to a dysfunction of the vestibular system and its interaction with other senses. This view has been confirmed in later studies, which identified disrupted activity in the TPJ to be associated with OBEs (Arzy et al., 2006; Blanke et al., 2002). The TPJ incorporates information from subcortical areas, like the thalamus and the limbic system, receives afferent from the visual, auditory and somatosensory system, and hosts the angular gyrus, a core region for the processing of vestibular signals and the coding of body orientation in space (Bottini et al., 1994; Brandt and Dieterich, 1999; Lopez et al., 2012). This is consistent with the established view that OBEs results from a failure to 
integrate somatosensory, visual and vestibular information into a coherent body representation (Blanke, 2012; Blanke and Mohr, 2005).

\subsection{BODY ILLUSIONS: EXPERIMENTAL TOOLS TO STUDY SELF-BODY PERCEPTION}

During bodily illusions healthy subjects are induced to experience altered body perceptions, which significantly deviates from the configuration of the real body and which could be very similar to the abnormal perceptions experienced in some of the neurological conditions involving dysfunctions of own-body perception. Several experimental paradigms have been developed and established in the last decades, which allow eliciting these illusions in controlled and reproducible experimental setups through the systematic manipulations of bodily stimuli. The consistency and reproducibility of the induced perceptual experiences, unachievable in neuropsychology, leveraged bodily illusions to be among the most powerful research tools in the study of own-body perception and its neural correlates.

In the following sections, a brief overview is given on the main experimental paradigms used in the scientific community for eliciting body illusions, and on the contribution that experimental studies utilizing body illusions have provided to the current understanding of how different bodily signals are processed and integrated to generate a unitary and stable own-body percept.

\subsubsection{Experimental Paradigms to Alter OWn-Body Perceptions}

Body illusions can be systematically induced in healthy subjects by manipulating multisensory bodily stimuli. The underlying principle is that, when presented with conflicting information about the body the brain generates abnormal body perceptions in the attempt to "make sense" of all incoming information. Depending on the nature of the conflict, different illusory experiences may arise, including illusory displacements of body parts or entire bodies, limb distortions, and ownership felt over fake objects resembling bodies or body parts.

Illusory body part displacements can be induced by dissociating visual and proprioceptive information about the location of a limb, by using prisms or mirrors to present a non veridical limb position (Hay et al., 1965). In these cases, vision dominates on proprioception as subjects typically misperceive their limb to be misplaced in the direction of the visual stimulus, an effect knows as visual capture of proprioception (van Beers et al., 1999). In absence of vision, the illusory movement of a static limb can be elicited by applying tendon vibration and results in a misperceived position of the limb (Goodwin et al., 1972). Illusory displacement of body parts, when combined with self-touch could elicit body distortion illusions. Touching one's own nose while experiencing an illusory extension of the elbow results in the sensation of an elongating Pinocchio-like nose (Lackner, 1988). In a similar fashion, touching one's own wrist while experiencing an illusory flexion of the wrist results in the striking sensation of a shrinking wrist (Ehrsson et al., 2005b).

Body ownership illusions pertain to a different class of bodily illusions and consist in the temporary attribution of external objects to the own-body. Sixty year after Tastevin (1937) described how an object protruding by one's clothes could be misperceived as one's own finger, Botvinick and Cohen (1998) introduced the Rubber Hand Illusion (RHI), highlighting its tight link with the multisensory 
processing of bodily stimuli and its great potential as an experimental tool. In the classic RHI, a rubber hand is placed on a table in front of the subject, while the real corresponding hand is hidden from view, and two paintbrushes are used to stroke simultaneously the real and the rubber hands. Under this simple, yet ingenious combination of conflictive sensory stimuli, most subjects report the feeling that the rubber hand is their own and that they feel touch as if generated by the paintbrush stroking it. Later studies introduced alternative setups to induce ownership illusions of hand shaped objects. The visual input from the non-corporeal hand (the rubber hand surrogate) could be given via mirrors (Longo et al., 2008), videos (Tsakiris et al., 2006) or virtual reality screens (Sanchez-Vives et al., 2010). These setups further allowed replacing the visuotactile stimulation, used in the classic RHI as the illusion trigger, with congruent visuomotor stimuli. Videos and virtual reality based setups allow in fact to display the non-corporeal hand replicating in real-rime the movements performed by the real hand, which can be filmed or tracked. It has been extensively shown that, besides these bottom-up multisensory components, other factors can modulate the illusory experiences in a top-down fashion. The visual appearance of the surrogate body with its level of realism (Maselli and Slater, 2013; Tsakiris et al., 2010), and the exposure to emotional or threatening stimuli during the illusion (Engelen et al., 2017) are representative examples. All these different setups, and many others, have been developed and adopted by a large number of research groups to selectively manipulate and explore the different factors potentially contributing to the experience of an external object as being part of one's own body and their impact on self-perception. An extensive review is given in Chapter 5 .

The introduction of immersive head mounted displays (HMD) has been decisive for extending the research work based on bodily illusions to encompass full body illusions and therefore to address issues related to self-consciousness (Blanke and Metzinger, 2009). In fact, the use of HMD entails the possibility - unworkable otherwise- to gather visual information from virtually all and every perspectives. These include disembodied perspectives, in which when looking down the space normally occupied by the own body is seen empty, and first-person perspectives on different bodies. These particular configurations of the visual perspective, in combination with specific multisensory stimulations, have been adopted for eliciting Full Body Ownership Illusions (FBOI) as well as illusory OBEs in healthy subjects.

The first FBOI study reported how healthy subjects could be induced to feel the body of a plastic mannequin as being their own body (Petkova and Ehrsson, 2008). Subjects were fitted with a HMD, completely occluding their own body, while streaming in real-time the video recorded from two cameras arranged at the eyes position of a plastic mannequin, pointing downward. The setup resulted in subjects having a static stereo view on the mannequin body, which visually replaced the real one. In analogy with the classic $\mathrm{RHI}$ protocol, experimenters were then able to induce a FBOI by stroking the real and the mannequin's chests simultaneously and with similar spatial patterns, while asynchronous stimulations resulted in no illusory experience. Few years later, a study based on immersive virtual reality technology showed how a similar FBOI could be induced over a virtual body, which could differ importantly from the participants body in both gender and age (Slater et al., 2010). In this study, participants could perform head movements that were tracked and used to accordingly render the scene in real time. Later studies showed how FBOls towards virtual bodies seen from a 1PP could be induced through visuomotor congruent stimuli, which is when participants are allowed to move and see the virtual body moving accordingly (González-Franco et al., 2010; 
Kokkinara and Slater, 2014; Spanlang et al., 2014). In the study presented in Chapter 2, it was further shown that, when the appearance of the virtual body is realistic, static visuoproprioceptive congruent stimuli (i.e., seeing the virtual body collocated with the real body) are sufficient to elicit FBOI (Maselli and Slater, 2013).

Illusory OBEs are another important class of full body illusions, in which illusory changes in selflocation are experienced. In this case, a HMD is used to occlude from view the real body and display it -or alternatively a virtual body-from a third person perspective (3PP) in the extra-personal space. The illusion is then triggered by applying visuotactile stimulation, analogously to the classic RHI and FBOI. Two different experimental manipulations have been introduced that can temporary altered the perceived self-location. Remarkably, the first reports of both types of experimentally induced OBE were published in same issue of the prestigious journal Science. In both cases, participants were fitted with a HMD streaming in stereo-view a video of their own body, captured by a videocamera placed two meters behind. In one case, a rod was used to stroke the real chest, while a similar rod was moved in the empty space right in front of the cameras, so that through the HMD the rod was seen moving in a congruent spatial location with respect to the felt touch, but from a disembodied 1PP perspective (Ehrsson, 2007b). In the second study, a rod was used to touch the back of the participant, who therefore felt it on the back while seeing moving on the back seen at in the extra-personal space from a 3PP (Lenggenhager et al., 2007). In the following, I will refer to these two variants as front-stroking and back-stroking experimentally induced OBE. The illusory experiences elicited with the two protocols differ from each other. While they both entail an illusory shift of the perceived self-location, they are characterized by different modulations of the sense of ownership. In front-stroking OBEs, an explicit sense of disownership with respect to the body seen in front is consistently reported (Ehrsson, 2007a; Guterstam and Ehrsson, 2012), while in back-stroking OBEs subjects tend to report a sense of self identification with the seen body (Aspell et al., 2009; lonta et al., 2011; Lenggenhager, 2007). However, it should be stressed that self-identification with a seen body is not necessarily equivalent to experiencing a sense of ownership of the same body (Maselli, 2015; Petkova and Ehrsson, 2008). The differences among the two types of illusory OBEs are extensively discussed in Chapters 6 and 7. Later studies have introduced alternative protocols to elicit illusory experiences similar to back-stroking OBEs. For example, it was recently shown that illusory OBEs can be induced experimentally with synchronous visuotactile stimuli delivered below detection threshold and therefore processed outside of awareness (Salomon et al., 2017).

\subsubsection{EXPERIMENTAL ASSESSMENTS AND MEASURES}

Measuring and quantifying subjective illusory experiences is not trivial. This is particularly true when dealing with illusory experiences like the feeling of ownership towards an external object, or the feeling of drifting in space away from the physical body. At first, characteristics of the qualitative experience of the illusion can be gathered through subjective reports. The use of questionnaires is the established practice to collect this kind of qualitative information in the form of ordinal data suited for comparison tests. However, explicit questionnaire items, such as "I felt as if the rubber hand were my hand", at interplay with the common tendency for task compliance, could result in biased and not reliable responses (Holtgraves, 2004; Slater, 2004). For this reason, questionnaire assessments should be complemented with other quantitative, although implicit, measurements. In fact, most of the research work on bodily illusions includes implicit assessments based on the monitoring of 
behavioural, physiological and/or neural correlates of the illusion.

Behavioural assessments of bodily illusions include implicit measurements of the spatial coding of body parts or of the whole body, responses to threats addressed to the fake body (part) and performances in psychophysics tasks, specifically designed to test the perceived spatiotemporal alignment of multisensory stimuli. In the case of the classic RHI, a blindfolded self-pointing task was used to show how subjects experiencing the illusion typically recode the position of their "substituted" hand towards the position of the rubber hand (Botvinick and Cohen, 1998). This effect, known as "proprioceptive drift", has been measured alternatively by asking participants to verbally report the perceived position of the hand with respect to hallmarks in the environment (Tsakiris and Haggard, 2005), and could alternatively be tested through reaching tasks (Holmes et al., 2004; Rossetti et al., 1995; Zopf et al., 2011b). Analogously, several methods have been introduced to measure illusory displacements of the whole body during OBE. These include the "walking task" in which participants had to reach their initial position after a random displacement guided by the experimenter (Lenggenhager, 2007), and a "ball dropping" task in which participants had to estimate the time for a ball held in their hand to reach the floor (lonta et al., 2011). These tasks were designed for measuring changes in the perceived self-location in a participant-centred egocentric reference frame. Other tasks were instead designed for assessing allocentric recodings of self-location, as for example asking participants to indicate their perceived location in a map of the experimental room (Guterstam and Ehrsson, 2012).

Alternative assessments of illusory changes in self-location, for both body parts and whole bodies, are based on experimental evaluations of the spatial structure of the peripersonal space (PPS). The PPS is classically defined as the "reachable" portion of space surrounding the body, in which object can be grasped and manipulated (Rizzolatti et al., 1981a, 1981b). This portion of space is represented by frontoparietal brain structures that integrate visual and auditory stimuli presented near the body with somatosensory information (di Pellegrino and Làdavas, 2015; Serino et al., 2015). The visual (or auditory) receptive fields of neurons in these areas are anchored to the corresponding part of the body encoded in the tactile domain. For example, a hand-encoding neuron of this type selectively responds to visual stimuli presented at different locations, depending on whether the hand is nearby (Graziano and Cooke, 2006).A standard tool to estimate the spatial extent of the PPS and its dynamic representation is the cross-modal congruency task (CCT), a psychophysics task designed to measure the effects of multisensory integration of independent stimuli presented through different sensory channels (Spence et al., 2008). In brief, the CCT efficacy as a tool for estimating the PPS extent in space in the visuotactile domain, is based on the evidence that reactions times to tactile target stimuli are modulated by the distance at which task-irrelevant visual stimuli are presented. In this way, it was shown how the felt position of the "replaced" hand(s) in the RHI shifts towards the location of the fake hand(s) (Pavani et al., 2000; Zopf et al., 2010). Similarly, CCT have been adopted to assess shifts in the perceived location of the full-body during illusory OBEs (Aspell et al., 2009; Maselli and Slater, 2014). A complementary audio-tactile paradigms, developed to infer the PPS extent through audio-tactile interactions (Canzoneri et al., 2012), was further adopted to reveal changes in the perceived self-location during OBEs illusions (Noel et al., 2015). 
Another quantitative measure of the illusory sense of ownership toward a rubber hand or a full fake body, consists in presenting an event threatening the fake body and in monitoring how the illusion modulates the evoked behavioural and physiological responses. During an ownership illusion, threatening events (e.g., suddenly bending back the rubber fingers in a painful posture or stabbing the rubber hand with a knife) elicit enhanced responses in skin conductance (Armel and Ramachandran, 2003) and enhanced activations of brain areas associated with anxiety (Ehrsson et al., 2007), which are not observed when the illusion is not experienced. Analogously, stabbing the chest of a mannequin triggers higher SCRs when a full body ownership illusion is experienced toward the mannequin, but not otherwise (Petkova and Ehrsson, 2008). Similarly, during the "frontstroking" induced OBEs illusions enhanced SCRs were recorded when hitting with a hammer the space of the illusory disembodied self (Ehrsson, 2007b).

A novel alternative measure for the sense of ownership towards a virtual body is based on behavioral assessments of the visuo-proprioceptive coupling that is established during a FBOI, and was introduced in the study presented in Chapter 6 (Maselli and Slater, 2014). The assessment consists in a behavioral measurement of the perceived body posture performed right after the posture of the surrogate body is virtually manipulated while the physical body stays still.The posture (visual) manipulation induces changes in the perceived posture of the own body when ownership is experience toward the virtual body, but not otherwise.

Physiological and neural correlates of bodily illusions have been explored in a large number of studies. Beside addressing the main goal of characterizing the origin and the neural mechanisms underlying bodily illusions, these works further served the function of providing objective and quantitative measures of illusory experiences. The illusory feeling of ownership over a rubber hand has several physiological effects measurable on the corresponding real hand, including cooling (Moseley et al., 2008a), reduced tactile (Zeller et al., 2015; Zopf et al., 2011a) and temperature (Llobera and Slater, 2013) sensitivity, and increased histamine reactivity (Barnsley et al., 2011). Brain imaging studies further revealed selective activation of distributed brain areas corresponding to specific aspects of bodily illusory experiences (see Section 1.2.3 for a detailed review). These selective patterns of activation could in turn, be regarded as objective markers of the specific illusory experience.

Importantly, all the objective measures reviewed above were shown to significantly correlate with the subjective ratings assessing the vividness of the illusory experience (Barnsley et al., 2011; Ehrsson et al., 2004; lonta et al., 2011; Limanowski and Blankenburg, 2015; Llobera and Slater, 2013; Moseley et al., 2008a). This quantitative link between the subjective quantification of illusory experiences, reflected in questionnaire ratings, and objective behavioural, physiological and neural measurements provides a strong evidence of the validity and robustness of bodily illusions as tools for quantitative research.

\subsubsection{Neural Correlates of Bodily Illusions}

Neuroimaging studies have shown how the patterns of brain activity engaged during bodily illusions depend on the specific aspects of the ongoing illusory experience. In all cases, imaging studies from different groups highlighted how bodily illusory experiences involve spread networks of brain activity, always including high-order multisensory areas known to encode the peripersonal space. The involvement of additional areas have been shown instead to reflect the specificity of the ongoing 
illusion, differentiating for example the illusory self-attribution of a body-part with respect to that of an entire artificial body, or illusory changes in body ownership (as in FBOI) from illusory changes in self-location (as in OBE illusions).

\section{Neural correlates of peripersonal space (PPS)}

The consistent engagement of multisensory areas associated with the encoding of peripersonal space (PPS) during bodily illusions is not surprising. In fact, the standard experimental paradigms used to trigger bodily illusions are based on the exposure to ambiguous multisensory bodily stimuli, which are eventually integrated giving rise to the illusory experience (Blanke, 2012; Ehrsson, 2011; Hohwy and Paton, 2010). Imaging fMRI studies in humans have revealed a set of multimodal areas integrating somatosensory information with external visual and auditory stimuli, homologous to those revealed by neurophysiological recordings in non-human primates (di Pellegrino and Làdavas, 2015). A set of interconnected areas encompassing the posterior parietal and premotor cortex was shown to encode the location of visual stimuli near the body in hand-centered coordinates (Brozzoli et al., 2011; Makin et al., 2007). Multisensory integration of touch on the hand with visual stimuli presented close to the hand were found to occur in a set of parietal (inferior posterior), frontal (dorsal and ventral premotor) and subcortical (cerebellum) regions (Gentile et al., 2011, 2013). Multisensory areas encoding different body parts in the visuotactile domain have been further detailed in a "higher-level" homunculus map within the posterior parietal cortex (Huang et al., 2012). So, while it is not surprising that activity in these areas is consistently implicated across different types of bodily illusions, specific combinations of co-activations and the involvement of additional areas were found to be specific of the nature of the illusory experience. In the follow, the neural correlates that have been identified for different types of bodily illusions are summarized.

\section{Self-attribution of body parts (RHI)}

The illusory self-attribution of a body part, as in the RHI, has been initially associated with enhanced activity in the ventral premotor cortex (vPM), which was found to correlate positively with the subjective ratings of the illusion (Ehrsson et al., 2004). In their seminal work, Ehrsson and colleagues further showed how other multisensory cortical (intraparietal sulcus and dorsal premotor - IPS and $\mathrm{dPM}$ ) and subcortical (cerebellum and putamen) regions show enhanced activity in experimental conditions leading to the illusion, but only in the early phase of the stimulation, i.e., before the illusion onset. Because of its specific temporal course, this activity -spread in various regions known to encode the PPS- was suggested to reflect the integration of (truly independent) sensory information and the related recalibration of the hand position. These conclusions were supported by later studies. Activity in the the vPM cortex was found to correlate with the rated strength of the illusion also in the somatic RHI (Ehrsson et al., 2005a), ruling out the possible confound that such activity was associated with the view of an object moving near the hand (Botvinick, 2004). This view of distinctive activities reflecting the recalibration of the hand location and the illusory sense of ownership of the rubber hand, is consistent with other following studies (Brozzoli et al., 2012; Tsakiris et al., 2007). By introducing an automatized device to deliver tactile stimuli that allows removing the view of another human (the experimenter), Limanowski and colleagues found that also the extrastriate body area (EBA) reflects the strength of the illusory experience (Limanowski et al., 2013; Limanowski and Blankenburg, 2015). Interestingly, these later studies further explored how brain activity is modulate at a network level.An increase in connectivity reflecting the illusory 
experience was observed between the EBA and multisensory parietal regions (IPC) as well as between EBA and unisensory regions (primary and secondary somatosensory cortex - SI and SII). The same group has next shown that activity in a similar set of brain areas (vPM, PPC and EBA) is enhanced also during the self-attribution of a rubber hand resulting by visuo-proprioceptive congruent information only, this time with an increased strength in the connection between EBA and the primary visual cortex (V1) (Limanowski and Blankenburg, 2016).

\section{Self attribution of entire bodies (FBOI)}

The self-attribution of entire bodies, as in FBOls, has been shown to involve the same multisensory areas implicated in the self-attribution of body parts, but with some specificity. Petkova and colleagues first reported enhanced activity in bilateral vPM, left IPC and left putamen during the illusion of owning an entire mannequin body (Petkova et al., 2011). Crucially, it was shown that activity in vPM cortex was independent on the specific body part exposed to the visuotactile stimulation and was correlated to the strength of the subjective illusory experience. Furthermore, activity in vPM was stronger if the body part receiving the visuotactile stimulation was seen attached to the body, a result the author interpreted as an evidence for the role of vPM in integrating multisensory information across body segments. However, it must be noticed that in the detached condition, the "detached" hand was shown isolated, with the rest of the body absent, so it is possible that the illusion was inhibited by the strong visuo-proprioceptive incongruence of seeing empty the space occupied by the physical body. Few years later, the same group showed how activity in the left vPM reflected the illusion of owning the whole body irrespectively of the site of the visuotactile stimulation (legs, hand or abomen), while other implicated multisensory areas (putamen, IPC, PM and lateral occipital cortex) were selectively implicated depending on the site of the stimulation (Gentile et al., 2015). These results support the established view that the feeling of owning an entire body, the whole-body percept, is mediated by neuronal populations that are hosted in the vPM and are characterized by visuo-somatic receptive fields encompassing different body segments.

\section{Illusory out-of-body experiences (OBEs)}

The neural correlates of experimentally induced OBEs have been investigated in a series of studies, carried out independently mainly by two groups. These studies have revealed how different brain networks underlie the illusory experiences elicited during front-stroking and back-stroking OBEs.

In the back-stroking paradigm, the illusory shifts in the perceived self-location towards the virtual body have been mainly associated with modulations in the TPJ and middle-inferior cortex activity, while the sense of self-identification with the virtual body have been associated with activity in the EBA (lonta et al., 2011). Interestingly levels of TPJ's activities were found to correlate with the perceived elevation of the body, when the back-stroking paradigm was applied to participants laying in the scanner and resulted in illusory shifts of self-location downward or upwards (lonta et al., 2011). Functional connectivity analysis revealed that illusory changes in the perceived self-location corresponded to enhanced connectivity patterns of the TPJ with a right-hemispheric networks including the insula and supplementary motor areas (lonta et al., 2014). EEGs recordings during standing back-stroking induced OBEs showed instead no major involvement of the TPJ, but revealed a positive correlation of the strength illusory shifts in self-location with the power of alpha-band oscillations in the medio-dorsal prefrontal cortex (Lenggenhager et al., 2011). 
The neural correlates of front-stroking induced OBEs includes different sets of brain areas. fMRI recordings revealed indeed the major involvement of hippocampus, posterior cingulate cortex (PCC), retrosplenial cortex (RSC) and intraparietal sulcus (IPS) (Guterstam et al., 2015). These regions are known to be relevant for spatial cognition, navigation (Vann et al., 2009) and mnemonic allocentric representations (Hassabis et al., 2009). The radical differences found in the brain activity monitored during front-stroking and back-stroking OBEs corroborates the differences emerging from subjective reports and behavioural correlates. The different nature of the two illusory experiences is discuss extensively in Chapter 7.

In summary, a consistent number of studies have demonstrated how extended network of multisensory brain areas underlie various aspects of own-body perception, with different subsets of brain areas selectively implicated in different types of bodily illusions. The fronto-partietal network corresponding to the multisensory representation of the space close to the body, i.e. the PPS, is always engaged, independently on the specific illusion under analysis. This is not surprising as the experimental protocols used to elicit bodily illusions rely on presenting multisensory bodily related stimuli as triggers of the illusory experience. Activations of different sets of brain areas specific to the type of illusion elicited, point instead to the multidimensional nature of the own-body percept.

\subsubsection{Impact of Body Illusions on Perception and Cognition}

Body illusions, even when transiently induced in the laboratory, are associated with a wide spectrum of perceptual and cognitive correlates. Profound implications of body change illusions have been demonstrated in a plethora of experimental studies, and encompass altered states that range from the processing of low-level sensory stimuli to cognitive processing and social behavior.

The illusory self-attribution of a body part, as experienced during a RHI, has an important impact on the ongoing processing of perceptual stimuli. It engenders a reduction of tactile sensitivity, demonstrated by increased reaction times (Folegatti et al., 2009), increased detection thresholds (Zopf et al., 2011a), and reduced amplitudes of somatosensory evoked potentials (Zeller et al., 2014), observed when tactile stimuli are delivered to participants undergoing a RHI, with respect to a non-illusory control condition. These effects are in line with complementary observations showing that the "substituted" hand's cools down during RHIs (Hohwy and Paton, 2010; Moseley et al., 2008a). This altered processing is compatible with the view that, during the illusion, the real hand is no longer processed as a part of the self. These observations are in line with the predictive coding models of multisensory integration, in that they may be explained as a way of minimizing sensory conflict (Seth et al., 2011; Zeller et al., 2015). In the study presented in Chapter 3, we showed how the impact of ownership illusions on perceptual processing extends to the domain of multisensory integration. The study demonstrated how, while undergoing a FBOI over a virtual body, the temporal constraints for multisensory integration of visuotactile stimuli are relaxed. This was interpreted as the result of the causal binding effect driven by the assumption that the visual and the tactile stimuli (seen on the virtual and felt on the physical body) have the same origin, i.e. the own-body (Maselli et al., 2016). Recently, it was shown how the sense of body ownership over a fake arm further involves the updating of the own-body representation used to plan actions and to predict their sensory consequences (Kilteni and Ehrsson, 2017).

The effects of body ownership illusions on the processing of afferent sensory signals extends to 
the perception of nociceptive stimuli. The studies that investigated the way ownership illusion could modulate pain perception mostly relied on the possibility to alter the physical properties of the fake body (part) that substitutes its real counterparts. These studies were in fact inspired by previous evidences showing that having view of one's own body when receiving a painful stimulus is analgesic (Longo et al., 2009), and that the pain sensitivity is further modulated by the perceived size of the body part affected (Mancini et al., 2011; Moseley et al., 2008b; Romano and Maravita, 2014). Along these lines, it was shown that analgesic effects (similar to those associated with viewing the real body part) are induced when viewing the surrogate hand during a RHI (Hegedüs et al., 2014; Martini et al., 2014), but only if the surrogate and the real hands are collocated (Nierula et al., 2017). It was further shown that pain thresholds could be modulated by manipulating the appearance of the fake arm. In particular, negative body image cues in the surrogate arm appearance - e.g., a reddened (Martini et al., 2013) or an injured arm (Osumi et al., 2014b) - were found to enhance pain sensitivity. An experimental study presented in Chapter 4, explored the possibility to modulate pain perception by varying the degree of transparency of a virtual body during FBOls, showing how pain thresholds increased with the level of ownership experienced toward semi-transparent virtual bodies (Martini et al., 2015). Beside paving the way to interesting clinical applications for pain treatments, these results provide further evidence for the complex interplay among the multisensory representation of the self-body, pain perception, and higher-order cognitive and emotional aspects associated with body appearance (Martini, 2016; Moseley et al., 2012; Romano and Maravita, 2014).

The use of immersive virtual reality has been crucial for exploring the impact of owning an artificial body, differing from one's own in terms of age, sex and race, on cognition and social aspects of behaviour. Several experiments have shown, for example, how white people undergoing the illusory experience of owning a black body, tend to reduce their implicit racial bias (Farmer et al., 2012; Maister et al., 2013; Peck et al., 2013). Experiencing embodiment over a child body has been shown to have important effects on both perception and behaviour. After embodying a child body, adults typically overestimate object sizes, enhance the implicit association of themselves with child-like attributes, and report positive changes in their emotional state (Banakou et al., 2013; TajaduraJiménez et al., 2017). Other studies have further demonstrated that body ownership illusions could further modulate the engagement and the actual performance on a given task, depending on the perceived appropriateness of the surrogate body for the task (Kilteni et al., 2013; Osimo et al., 2015). Finally, a recent work showed how being embodied in a female body while receiving a verbal domestic abuse by a male avatar could improve the ability of male offenders to properly process fearful faces (Seinfeld et al., 2018). Interestingly, it was further showed that the experience of full body ownership illusion can have significant after-effects on the way threatening stimuli are processed. In particular, a recent study showed that experiencing an ownership illusion over a female character modulates the activation of defensive and emotional processing, in fronto-parietal networks encoding human peripersonal space, during the subsequent exposure to a verbal aggression by a virtual male offender (de Borst et al., 2018).

The results reviewed in this section provide just a rough overview of the profound impact that illusory experience of body ownership over fake bodies can have on perception, behaviour and cognition, over time scales of few tens of seconds. Nevertheless, it appears evident from this overview that, beside providing a powerful tool for scientific research, bodily illusions proved to have a great 
potential for application in the clinical, therapeutic and educational applications.

\section{StRucture AND CONTRIBUtion OF THESIS}

The works presented in this thesis focus on the study of the multisensory mechanisms underlying the perception of our own bodies. By making use of Immersive Virtual Reality and established experimental paradigms for eliciting bodily illusions, the multisensory basis of two main components of self-perception, the sense of body ownership and self-location, have been identified. In addition, some implications of manipulating ownership under controlled experimental settings have been explored.The first part of the thesis (Chapters 2-5) deals with sense of ownership experienced in full body ownership illusions (FBOls). The second part (Chapters 6 and 7) explores how the sense of ownership and self-location differ from each other, how they can be selectively altered during bodily illusions, and how they are differently characterized in terms of subjective perceptual experience and multisensory mechanisms involved.

The aim of the study presented in Chapter $\mathbf{2}$ was to identify the perceptual "building blocks" of the sense of body ownership in terms of the major multisensory components involved and of their mutual interaction. The study was initially planned for disambiguating earlier reports about whether or not congruent visuotactile stimulation is a necessary trigger for eliciting a FBOI (Petkova and Ehrsson 2007 vs Slater et al. 2010), but eventually it was designed to more generally pinpoint the minimal combinations of multisensory stimuli that could elicit a FBOI. More specifically, we explored the role and mutual interaction of different perceptual components that have been manipulated in previous experimental studies on FBOls. These were: (i) the degree of realism of the surrogate body (mannequin vs realistic gender-matched human body), (ii) the visual perspective over the surrogate body (1PP vs 3PP), (iii) the congruency of visuotactile stimuli (synchronous vs asynchronous) and (iv) the availability of visuo-vestibular signals associated to head movements (present or absent). By manipulating these components while monitoring the level of the induced FBOI in healthy participants via subjective reports and physiological correlates, we could achieve several novel insights into the mechanisms that allow to swap the sense of body ownership from our physical one to a virtual one.These results, presented and discussed in Chapter 2, provided the bases for the Bayesian inference formulation for describing the mechanisms that determine whether or not an external object is experienced as belonging to the own-body, which was formulated and presented in a following study (Chapter 5).

The study presented in Chapter 3 tested the following hypothesis: when undergoing an ownership illusion the temporal constraints for visuotactile integration are relaxed as a result of the causal binding between the real and the fake bodies established by the illusion. This hypothesis stemmed from a serendipitous finding from our previous study, in which we found that when the FBOI was established, asynchronous visuotactile stimuli were not perceived as "wrong", which is otherwise the case (presented in Chapter 2). Indeed this seemed to suggest that during ownership illusions participants are more tolerant to visuotactile incongruences. To test this idea, we designed an experimental protocol combining the triggering of a FBOI and a temporal order judgment (TOJ) 
task. The latter allowed psychophysical quantification of the temporal window for visuotactile integration to occur (Vroomen and Keetels, 2010). Participants in this experiment performed the same TOJ task in three different conditions in which we manipulated the sense of ownership and the visual information providing, or not, hints for the visual and tactile stimuli in the TOJ to be causally related. We found that the temporal window for visuotactile integration was significantly larger when participants experienced an ownership illusion and the visual stimuli were consistent with an object touching the virtual body, with respect to other conditions. Crucially, the temporal window for visuotactile integration was positively correlated with the strength of the ownership illusions measured through questionnaire ratings.

In Chapter 4 a study is presented that, taking advantage of the possibility to arbitrarily manipulate the appearance of a virtual body, investigated how the level of transparency of a virtual body affects the sense of body ownership and pain perception in healthy participants. The study was motivated by a set of previous experimental evidences. Having direct view of one's own body while receiving painful stimuli was shown to have an analgesic effect (Longo et al., 2009), an effect that holds true when looking at a collocated virtual body during ownership illusions (Martini et al., 2014; Nierula et al., 2017). Complementary evidence showed that perception of painful stimuli on the hand is modulated when manipulating the appearance of the hand, either in size (Mancini et al., 2011) or skin colour (Martini et al., 2013). In line with our previous study showing how the level of realism of the virtual body modulates the strength of FBOls (Maselli and Slater, 2013), we expected that by gradually decreasing the visibility of the virtual body the experienced sense of ownership would fade away. On the other hand, we could not make straightforward predictions about the impact on pain perception. Would the analgesic effect fade away together with the vision of the body that in normal condition produce it? Or otherwise, would the analgesic effect be modulated by the cognitive value of having a transparent body? The results from our experiment showed that, as expected the strength of perceived ownership over the collocated virtual body seen from 1PP diminished progressively for increasing levels of body transparency. On the other hand, we found that the level of transparency does not directly modulate pain perception. Nevertheless, we found that in the conditions where the body was semi-transparent, higher levels of ownership resulted in an increased pain sensitivity. Different interpretations for these finding have been thoroughly discussed.

Chapter 5 presents a general overview of "how and why" body ownership illusions (BOls) occur. The perspective taken was to address the following question: how does our brain distinguish its own body from others' bodies and objects in the environment? To tackle this question, we first undertook a broad review of experimental findings concerning the spatial, temporal, and semantic principles of crossmodal stimuli used to trigger BOls, presenting them in a coherent and unified scheme. We did so by analysing the semantic and spatiotemporal constraints that different multimodal triggers (visuotactile, visuomotor and visuoproprioceptive) need to satisfy for eliciting BOls. We further reviewed the theoretical frameworks that have been proposed to describe the underlying mechanism of BOls. Next, based on the schematic arrangement we proposed for the available experimental evidences, we introduced a conceptual model for how our nervous system infers whether an object belongs to the body, giving rise in some cases to BOls. The model is worked out in the Bayesian inference framework for the representative case of the classic RHI.After presenting our formulation, we discussed how it can account for several experimental findings. Finally, we discussed how neural 
network models already developed in the literature for multisensory integration can be taken as implementational frameworks within which the computational problem behind BOls could be addressed in the future.

The last two chapters of this thesis explore how, while intrinsically interlaced in normal body perception, the sense of ownership and the sense of self-location can be selectively altered during FBOls.

Chapter 6 presents an experimental study in which the aim was to test to what extent changes in self-location entail changes in ownership. The lack of consensus about this issue in previous works from different groups was the main motivation for conducting the study. In fact, on the one hand in our previous study we confirmed that illusory ownership over a fake body required having a first person perspective (1PP) on it and an established visuoproprioceptive coupling between the real and fake bodies (Maselli and Slater, 2013). On the other, independent works consistently reported that ownership towards a filmed or virtual body was experienced together with changes in selflocation during illusory back-stroking OBEs (Blanke, 2012; lonta et al., 2011; Lenggenhager, 2007). However, in these latter studies ownership was assessed only through questionnaire scores that, as we reasoned, could reflect a sense of self-identification rather than actual ownership. We therefore designed a set of experiments in which FBOls were triggered from different visual perspectives, each corresponding to different degree of overlap between real and virtual bodies. Concurrently both ownership and self-location were measured using a combination of questionnaire, psychophysical and behavioural assessments. A novel self-posture task was introduced to measure ownership by assessing the strength of visuoproprioceptive coupling, while an adaptation of the cross-modal congruency task was adopted to indirectly measure self-location. We found that, despite their strong coupling, self-location and ownership were selectively altered depending on the perspective. The results demonstrated that illusory OBEs are mainly associated with alterations in the perceived sense of self-location, and do not necessarily involve alterations in the sense of body ownership. When discussing our results in the context of the relevant literature, we argued that changes in the perceived self-location are associated to the dynamical representation of peripersonal space encoded by visuotactile neurons, while illusory ownership entails the implication of visuoproprioceptive populations.

Finally, Chapter 7 provides a closer look into the nuances of different illusory experiences affecting the sense of self-location and their relation to ownership. The ideas developed in Chapter 6 have been extended and complemented to further disentangle how self-location is differently modulated in front-stroking and back-stroking OBEs. The profound differences between the two illusory experiences that a number of studies had previously highlighted, at the level of subjective experience and of behavioural and neural correlates, have been attentively described. Based on these, it was proposed that the sense of self-location may be regarded as the blending of two parallel representations: the abstract allocentric coding of the position occupied in the environment, and the egocentric mapping of somatosensory sensations into the external space. While the first is mainly associated with visual-perspective, the second is tightly linked to peripersonal space. 


\section{RefERENCES}

Armel, K. C., and Ramachandran, V. S. (2003). Projecting sensations to external objects: evidence from skin conductance response. Proc. Biol. Sci. 270, 1499-506

Arzy, S., Thut, G., Mohr, C., Michel, C. M., and Blanke, O. (2006). Neural basis of embodiment: distinct contributions of temporoparietal junction and extrastriate body area. J. Neurosci. 26, 8074-81

Aspell, J. E., Lenggenhager, B., and Blanke, O. (2009). Keeping in touch with one's self: multisensory mechanisms of self-consciousness. PLoS One 4, e6488

Baier, B., and Karnath, H.-O. (2008). Tight link between our sense of limb ownership and self-awareness of actions. Stroke. $39,486-8$

Banakou, D., Groten, R., and Slater, M. (2013). Illusory ownership of a virtual child body causes overestimation of object sizes and implicit attitude changes. Proc. Natl. Acad. Sci. U. S.A. 110, 12846-12851

Barnsley, N., McAuley, J. H., Mohan, R., Dey, a.,Thomas, P., and Moseley, G. L. (2011). The rubber hand illusion increases histamine reactivity in the real arm. Curr. Biol. 21, R945-R946.

Berlucchi, G., and Aglioti, S. (1997). The body in the brain: Neural bases of corporeal awareness. Trends Neurosci. 20, 560-564.

Berlucchi, G., and Aglioti, S. M. (2010). The body in the brain revisited. Exp. Brain Res. 200, 25-35.

Bisiach, E., Rusconi, M. L., and Vallar, G. (1991). Remission of somatoparaphrenic delusion through vestibular stimulation. Neuropsycholo 29, 1029-1031.

Blackmore, S. J. (1982). Beyond the Body: An Investigation of out-of-the-Body Experiences. Heinmann. London.

Blanke, O. (2012). Multisensory brain mechanisms of bodily self-consciousness. Nat. Rev. Neurosci. 13, 556-71.

Blanke, O., and Arzy, S. (2005). The out-of-body experience: disturbed self-processing at the temporo-parietal junction. Neuroscientist 11, 16-24

Blanke, O., and Metzinger, T. (2009). Full-body illusions and minimal phenomenal selfhood. Trends Cogn. Sci. 13, 7-13.

Blanke, O., and Mohr, C. (2005). Out-of-body experience, heautoscopy, and autoscopic hallucination of neurological origin Implications for neurocognitive mechanisms of corporeal awareness and self-consciousness. Brain Res. Brain Res. Rev. $50,184-99$.

Blanke, O., Ortigue, S., Landis, T., and Seeck, M. (2002). Stimulating illusory own-body perceptions. Nature 419, 26970.

Bottini, G., Sterzi, R., Paulesu, E., Vallar, G., Cappa, S. F., Erminio, F., et al. (1994). Identification of the central vestibular projections in man: a positron emission tomography activation study. Exp. Brain Res. 99, 164-169.

Botvinick, M. (2004). Probing the Neural Basis of Body Ownership. Science 305, 782-783.

Botvinick, M., and Cohen, J. (1998). Rubber hands "feel" touch that eyes see. Nature 391, 756 .

Brandt, T., and Dieterich, M. (1999). The Vestibular Cortex: Its Locations, Functions and Disorders. Ann. N.Y. Acad. Sci. 871, 293-312.

Brozzoli, C., Gentile, G., and Ehrsson, H. H. (2012). That's near my hand! Parietal and premotor coding of hand-centered space contributes to localization and self-attribution of the hand. J. Neurosci. 32, 14573-82.

Brozzoli, C., Gentile, G., Petkova, V. I., and Ehrsson, H. H. (2011). FMRI adaptation reveals a cortical mechanism for the coding of space near the hand. J. Neurosci. 31, 9023-31.

Brugger, P., Regard, M., and Landis, T. (1997). Illusory Reduplication of One's Own Body: Phenomenology and Classification of Autoscopic Phenomena. Cogn. Neuropsychiatry 2,19-38.

Canzoneri, E., Magosso, E., and Serino, A. (2012). Dynamic sounds capture the boundaries of peripersonal space representation in humans. PLoS One 7, e44306.

Critchley, M. (1955). Personification of Paralysed Limbs in Hemiplegics. Br. Med. J. 2, 490-491.

de Borst, A. W., Sanchez-Vives, M. V., Slater, M., and de Gelder, B. (2018). First person experience of threat modulates cortical network encoding human peripersonal space. bioRxiv. doi:http://dx.doi.org/10.1101/314971 
de Gelder, B., Kätsyri, J., and de Borst, A.W. (2018). Virtual reality and the new psychophysics. Br. J. Psychol., 1-6.

De Ridder, D., Van Laere, K., Dupont, P., Menovsky, T., and Van de Heyning, P. (2007). Visualizing out-of-body experience in the brain. N. Engl. J. Med. 357, 1829-1833. doi:10.1056/ NEJMoa070010.

Devinsky, O., Feldmann, E., Burrowes, K., and Bromfield, E. (1989). Autoscopic phenomena with seizures. Arch. Neurol. 46, 1080-1088.

di Pellegrino, G., and Làdavas, E. (2015). Peripersonal space in the brain. Neuropsychologia 66,126-133.

Ehrsson, H. H. (2007a). The experimental induction of out-ofbody experiences. Science (80-. ). 317, 1048.

Ehrsson, H. H. (2007b). The experimental induction of out-ofbody experiences. Science 317, 1048.

Ehrsson, H. H. (2011). "The Concept of Body Ownership and Its Relation to Multisensory Integration," in The New Handbook of Multisensory Processes, ed. B. E. Stein (Cambridge: MIT Press), 775-792.

Ehrsson, H. H., Holmes, N. P., and Passingham, R. E. (2005a). Touching a rubber hand: feeling of body ownership is associated with activity in multisensory brain areas. J. Neurosci. 25, 10564-73.

Ehrsson, H. H., Kito, T., Sadato, N., Passingham, R. E., and Naito, E. (2005b). Neural substrate of body size: illusory feeling of shrinking of the waist. PLoS Biol. 3, e412.

Ehrsson, H. H., Spence, C., and Passingham, R. E. (2004). That's my hand! Activity in premotor cortex reflects feeling of ownership of a limb. Science 305, 875-877.

Ehrsson, H. H., Wiech, K., Weiskopf, N., Dolan, R. J., and Passingham, R. E. (2007). Threatening a rubber hand that you feel is yours elicits a cortical anxiety response. Proc. Natl.Acad. Sci. U. S.A. 104, 9828-33.

Engelen, T., Watson, R., Pavani, F., and de Gelder, B. (2017). Affective vocalizations influence body ownership as measured in the rubber hand illusion. PLoS One 12,1-11.

Farmer, H., Tajadura-Jiménez, A., and Tsakiris, M. (2012). Beyond the colour of my skin: How skin colour affects the sense of body-ownership. Conscious. Cogn. 21, 1242-1256.

Feinberg, T. E. (2010). Neuropathologies of the Self: A General Theory. Neuropsychoanalysis 12,133-158.

Feinberg, T. E., and Roane, D. M. (2005). Delusional misidentification. Psychiatr. Clin. North Am. 28, 665-83, 6789. doi:10.1016/j.psc.2005.05.002.

Feinberg, T. E., and Venneri, A. (2014). Somatoparaphrenia: Evolving theories and concepts. Cortex 61, 74-80.

Feinberg, T. E., Venneri, A., Simone, A. M., Fan, Y., and Northoff, G. (2010). The neuroanatomy of asomatognosia and somatoparaphrenia. J. Neurol. Neurosurg. Psychiatry 81, 276-81.

Folegatti, A., de Vignemont, F., Pavani, F., Rossetti, Y., and Farnè,A. (2009). Losing one's hand: visual-proprioceptive conflict affects touch perception. PLoS One 4, e6920.

Gandola, M., Invernizzi, P., Sedda, A., Ferrè, E. R., Sterzi, R., Sberna, M., et al. (2012). An anatomical account of somatoparaphrenia. Cortex. 48, 1165-78.

Gentile, G., Björnsdotter, M., Petkova, V. I., Abdulkarim, Z., and Ehrsson, H. H. (2015). Patterns of neural activity in the human ventral premotor cortex reflect $a$ whole-body multisensory percept. Neuroimage 109, 328-340.

Gentile, G., Guterstam, A., Brozzoli, C., and Ehrsson, H. H. (2013). Disintegration of multisensory signals from the real hand reduces default limb self-attribution: an FMRI study. J. Neurosci. 33, 13350-66.

Gentile, G.,Petkova,V.I., and Ehrsson, H.H. (2011). Integration of visual and tactile signals from the hand in the human brain: an FMRI study. J. Neurophysiol. 105, 910-22.

Gerstmann, J. (1942). Problem of imperception of disease and of impaired body territories with organic lesions: Relation to body scheme and its disorders. Arch. Neurol. Psychiatry 48, 890-913.

González-Franco, M., Pérez-marcos, D., Spanlang, B., and Slater, M. (2010). The Contribution of Real-Time Mirror Reflections of Motor Actions on Virtual Body Ownership in an Immersive Virtual Environment. IEEE Virtual Real., 111-114.

Goodwin, G. M., McCloskey, D. I., and Matthews, P. B. C. 
(1972). Proprioceptive illusions induced by muscle vibration: contribution by muscle spindles to perception? Science (80). 175, 1382-1384.

Graziano, M. S. A., and Cooke, D. F. (2006). Parietofrontal interactions, personal space, and defensive behavior. Neuropsychologia 44, 845-859.

Guterstam, A., Björnsdotter, M., Gentile, G., and Ehrsson, H. H. (2015). Posterior Cingulate Cortex Integrates the Senses of Self-Location and Body Ownership. Curr. Biol. 25, 1-10.

Guterstam, A., and Ehrsson, H. H. (2012). Disowning one's seen real body during an out-of-body illusion. Conscious. Cogn. 21, 1037-42.

Haggard, P., Clark, S., and Kalogeras, J. (2002). Voluntary action and conscious awareness. Nat. Neurosci. 5, 382-385. doi:10.1038/nn827.

Haggard, P., lannetti, G. D., and Longo, M. R. (2013). Spatial sensory organization and body representation in pain perception. Curr. Biol. 23, R164-R176.

Harrar, V., and Harris, L. R. (2005). Simultaneity constancy: detecting events with touch and vision. Exp. brain Res. 166, 465-73.

Hassabis, D., Chu, C., Rees, G., Weiskopf, N., Molyneux, P. D., and Maguire, E. a. (2009). Decoding Neuronal Ensembles in the Human Hippocampus. Curr. Biol. 19, 546-554.

Hay, J., Pick, H., and Ikeda, K. (1965). Visual capture produced by prism spectacles. Psychon. Sci. 2, 215-216.

Head, H., and Holmes, G. (1911). Sensory Disturbances from Cerebral Lesions. Brain 34, 102-254.

Hegedüs, G., Darnai, G., Szolcsányi, T., Feldmann, A., Janszky, J., and Kállai, J. (2014). The rubber hand illusion increases heat pain threshold. Eur. J. Pain (United Kingdom) 18, 1173-1181.

Hilti, L. M., Hänggi, J., Vitacco, D. A., Kraemer, B., Palla, A., Luechinger, R., et al. (2013). The desire for healthy limb amputation: Structural brain correlates and clinical features of xenomelia. Brain 136, 318-329.

Hohwy, J., and Paton, B. (2010). Explaining away the body: experiences of supernaturally caused touch and touch on nonhand objects within the rubber hand illusion. PLoS One 5, e9416.
Holmes, N. P., Crozier, G., and Spence, C. (2004). When mirrors lie :"Visual capture " of arm position impairs reaching performance. Cogn.Affect. Behav. Neurosci. 4, 193-200.

Holtgraves, T. (2004). Social Desirability and Self-Reports: Testing Models of Socially Desirable Responding. Personal. Soc. Psychol. Bull. 30, 161-172.

Huang, R.-S., Chen, C., Tran,A.T., Holstein, K. L., and Sereno, M. I. (2012). Mapping multisensory parietal face and body areas in humans. Proc. Natl.Acad. Sci. U. S.A. 109, 18114-9.

Invernizzi, P., Gandola, M., Romano, D., Zapparoli, L., Bottini, G., and Paulesu, E. (2013). What is mine? Behavioral and anatomical dissociations between somatoparaphrenia and anosognosia for hemiplegia. Behav. Neurol. 26, 139-150.

Ionta, S., Heydrich, L., Lenggenhager, B., Mouthon, M., Fornari, E., Chapuis, D., et al. (2011). Multisensory mechanisms in temporo-parietal cortex support self-location and first-person perspective. Neuron 70, 363-74.

lonta, S., Martuzzi, R., Salomon, R., and Blanke, O. (2014). The brain network reflecting bodily self-consciousness: $a$ functional connectivity study. Soc. Cogn. Affect. Neurosci., 1-10.

Kilteni, K., Bergstrom, I., and Slater, M. (2013). Drumming in immersive virtual reality: the body shapes the way we play. IEEE Trans.Vis. Comput. Graph. 19, 597-605.

Kilteni, K., and Ehrsson, H. H. (2017). Body ownership determines the attenuation of self-generated tactile sensations. Proc. Natl.Acad. Sci. 114, 8426-8431.

Kokkinara, E., and Slater, M. (2014). Measuring the effects through time of the influence of visuomotor and visuotactile synchronous stimulation on a virtual body ownership illusion. Perception 43, 43-58.

Kopinska,A., and Harris, L. R. (2004). Simultaneity constancy. Perception 33, 1049-1060

Lackner, J. R. (1988). Some proprioceptive influences on the perceptual representation of body shape and orientation. Brain 111, 281-297.

Lenggenhager, B. (2007). Video Ergo Sum: Manipulating Bodily. Science, 80, 1095-1099.

Lenggenhager, B., Halje, P., and Blanke, O. (2011). Alpha 
band oscillations correlate with illusory self-location induced by virtual reality. Eur. J. Neurosci. 33, 1935-43.

Limanowski, J., and Blankenburg, F. (2015). Network activity underlying the illusory self-attribution of a dummy arm. Hum. Brain Mapp. 36, 2284-2304

Limanowski, J., and Blankenburg, F. (2016). Integration of Visual and Proprioceptive Limb Position Information in Human Posterior Parietal, Premotor, and Extrastriate Cortex. J. Neurosci. 36, 2582-2589. doi:10.1523/JNEUROSCI.3987-15.2016.

Limanowski, J., Lutti, A., and Blankenburg, F. (2013). The extrastriate body area is involved in illusory limb ownership. Neuroimage 86, 514-524.

Lippman, C. W. (1953). Hallucinations of Physical Duality in Migraine. J. Nerv. Ment. Dis. 117, 345-350.

Llobera, J., and Slater, M. (2013). The relationship between virtual body ownership and temperature sensitivity. J R Soc Interface 10: 20130300.

Longo, M. R., Betti, V., Aglioti, S. M., and Haggard, P. (2009). Visually induced analgesia: seeing the body reduces pain. J. Neurosci. 29, 12125-30.

Longo, M. R., Cardozo, S., and Haggard, P. (2008). Visual enhancement of touch and the bodily self. Conscious. Cogn. 17, 1181-91.

Longo, M. R., lannetti, G. D., Mancini, F., Driver, J., and Haggard, P. (2012). Linking pain and the body: neural correlates of visually induced analgesia. J. Neurosci. 32, 2601-7.

Lopez, C., Blanke, O., and Mast, F.W. (2012). The human vestibular cortex revealed by coordinate-based activation likelihood estimation meta-analysis. Neuroscience 212, 159 79.

Lopez, C., and Elzière, M. (2017). Out-of-body experience in vestibular disorders - A prospective study of 210 patients with dizziness. Cortex.

Maister, L., Sebanz, N., Knoblich, G., and Tsakiris, M. (2013). Experiencing ownership over a dark-skinned body reduces implicit racial bias. Cognition 128, 170-178.

Makin, T. R., Holmes, N. P., and Zohary, E. (2007). Is that near my hand? Multisensory representation of peripersonal space in human intraparietal sulcus. J. Neurosci. 27, 731-40.
Mancini, F., Longo, M. R., Kammers, M. P. M., and Haggard, P. (2011). Visual distortion of body size modulates pain perception. Psychol. Sci. 22, 325-330.

Martini, M. (2016). Real, rubber or virtual:The vision of "one's own" body as a means for pain modulation. A narrative review. Conscious. Cogn. 43, 143-151.

Martini, M., Kilteni, K., Maselli, A., and Sanchez-vives, M. $\checkmark$ (2015). The body fades away: investigating the effects of transparency of an embodied virtual body on pain threshold and body ownership. Sci. Rep. 5, 13948.

Martini, M., Perez-Marcos, D., and Sanchez-Vives, M. V. (2013). What Color is My Arm? Changes in Skin Color of an Embodied Virtual Arm Modulates Pain Threshold. Front. Hum. Neurosci. 7, 438.

Martini, M., Perez-Marcos, D., and Sanchez-Vives, M. V. (2014). Modulation of pain threshold by virtual body ownership. Eur. J. Pain (United Kingdom) 18, 1040-1048.

Maselli, A. (2015). Allocentric and egocentric manipulations of the sense of self-location in full-body illusions and their relation with the sense of body ownership. Cogn. Process. 16.

Maselli,A., Kilteni, K., López-Moliner, J., and Slater, M. (2016). The sense of body ownership relaxes temporal constraints for multisensory integration. Sci. Rep. 6, 30628.

Maselli,A., and Slater, M. (2013). The building blocks of the full body ownership illusion. Front. Hum. Neurosci. 7, 83.

Maselli, A., and Slater, M. (2014). Sliding perspectives: dissociating ownership from self-location during full body illusions in virtual reality. Front. Hum. Neurosci. 8, 693.

Melzack, R. (1990). Phantom limbs and the concept of $a$ neuromatrix. Trends Neurosci. 13, 88-92.

Moseley, G. L., Gallace, A., and Spence, C. (2012). Bodily illusions in health and disease: physiological and clinical perspectives and the concept of a cortical "body matrix". Neurosci. Biobehav. Rev. 36, 34-46.

Moseley, G. L., Olthof, N., Venema, A., Don, S., Wijers, M., Gallace, A., et al. (2008a). Psychologically induced cooling of a specific body part caused by the illusory ownership of an artificial counterpart. Proc. Natl.Acad. Sci. U.S.A. 105, 1316973.

Moseley, G. L., Parsons, T. J., and Spence, C. (2008b). Visual 
distortion of a limb modulates the pain and ing evoked by movement. Curr. Biol. 18, 1047-1048.

Nierula, B., Martini, M., Matamala-Gomez, M., Slater, M., and Sanchez-Vives, M.V. (2017). Seeing an Embodied Virtual Hand is Analgesic Contingent on Colocation. J. Pain 18, 645655.

Noel, J.-P., Pfeiffer, C., Blanke, O., and Serino, A. (2015). Peripersonal space as the space of the bodily self. Cognition 144, 49-57

Osimo, S. A., Pizarro, R., Spanlang, B., and Slater, M. (2015). Conversations between self and self as Sigmund Freud-A virtual body ownership paradigm for self counselling. Sci. Rep. 5,13899 .

Osumi, M., Imai, R., Ueta, K., Nakano, H., Nobusako, S., and Morioka, S. (2014a). Factors associated with the modulation of pain by visual distortion of body size. Front. Hum. Neurosci. $8,137$.

Osumi, M., Imai, R., Ueta, K., Nobusako, S., and Morioka, S. (2014b). Negative body image associated with changes in the visual body appearance increases pain perception. PLoS One 9, e107376.

Pavani, F., Spence, C., and Driver, J. (2000). Visual capture of touch : out-of-the-body experiences with rubber gloves. Psychol. Sci. 11, 353-359.

Peck, T. C., Seinfeld, S., Aglioti, S. M., and Slater, M. (2013). Putting yourself in the skin of a black avatar reduces implicit racial bias. Conscious. Cogn. 22, 779-787.

Petkova, V. I., Björnsdotter, M., Gentile, G., Jonsson, T., Li, T., and Ehrsson, H. H. (2011). From part- to whole-body ownership in the multisensory brain. Curr. Biol. 21, 1118-22.

Petkova, V. I., and Ehrsson, H. H. (2008). If I were you: perceptual illusion of body swapping. PLoS One 3, e3832.

Ramachandran,V.S., and Hirstein,W. (1998). The perception of phantom limbs. Brain 121, 1603-1630.

Rizzolatti, G., Scandarola, C., Matelli, M., and Gentilucci, M. (1981a). Afferent properties of periarcuate neurons in macaque monkeys. II.Visual responses. Behav. Brain Res. 2, 147-163.

Rizzolatti, G., Scandolara, C., Matelli, M., and Gentilucci, M. (1981b). Afferent properties of periarcuate neurons in macaque monkeys. I. Somatosensory responses. Behav. Brain Res. 2,
$125-146$.

Romano,D., Gandola,M.,Bottini, G., and Maravita,A.(2014). Arousal responses to noxious stimuli in somatoparaphrenia and anosognosia: Clues to body awareness. Brain 137, 1213-1223

Romano, D., and Maravita, A. (2014). The visual size of one's own hand modulates pain anticipation and perception. Neuropsychologia 57, 93-100.

Rossetti, Y., Desmurget, M., and Prablanc, C. (1995). Vectorial Coding of Movement: Vision, Proprioception, or Both? J. Neuropsysiology 74, 457-463.

Salomon, R., Noel, J. P., Łukowska, M., Faivre, N., Metzinger, T., Serino, A., et al. (2017). Unconscious integration of multisensory bodily inputs in the peripersonal space shapes bodily self-consciousness. Cognition 166, 174-183.

Sanchez-Vives, M. V., and Slater, M. (2005). Opinion: From presence to consciousness through virtual reality. Nat. Rev. Neurosci. 6, 332-339.

Sanchez-Vives, M. V, Spanlang, B., Frisoli, A., Bergamasco, M., and Slater, M. (2010). Virtual hand illusion induced by visuomotor correlations. PLoS One 5, e10381.

Seinfeld, S., Arroyo-Palacios, J., Iruretagoyena, G., Hortensius, R., Zapata, L. E., Borland, D., et al. (2018). Offenders become the victim in virtual reality: impact of changing perspective in domestic violence. Sci. Rep. 8, 2692.

Serino, A., Noel, J. P., Galli, G., Canzoneri, E., Marmaroli, P., Lissek, H., et al. (2015). Body part-centered and full bodycentered peripersonal space representations. Sci. Rep. 5, 1-14.

Seth, A. K., Suzuki, K., and Critchley, H. D. (2011). An interoceptive predictive coding model of conscious presence. Front. Psychol. 2, 395.

Slater, M. (2004). How Colorful Was Your Day? Why Questionnaires Cannot Assess Presence in. Presence 13, 484493.

Slater, M., Spanlang, B., Sanchez-Vives, M. V, and Blanke, O. (2010). First person experience of body transfer in virtual reality. PLoS One 5, e10564.

Spanlang, B., Normand, J.-M., Borland, D., Kilteni, K., Giannopoulos, E., Pomés, A., et al. (2014). How to Build an Embodiment Lab: Achieving Body Representation Illusions in Virtual Reality. Front. Robot. Al 1,1-22. 
Spence, C., Pavani, F., Maravita, A., and Holmes, N. P. (2008). "Multi-Sensory Interactions," in Haptic rendering: Foundations, algorithms, and applications, eds. M. C. Lin and M.A. Otaduy (Wellesley, MA:AK Peters), 21-52.

Tajadura-Jiménez, A., Banakou, D., Bianchi-Berthouze, N., and Slater, M. (2017). Embodiment in a Child-Like Talking Virtual Body Influences Object Size Perception, Self-Identification, and Subsequent Real Speaking. Sci. Rep. 7,1-12.

Tastevin, J. (1937). En partant de l'experience d'Aristotle [Starting from Aristotle's experience]. L'Encephale 1, 140-158.

Tieri, G., Tidoni, E., Pavone, E. F., and Aglioti, S. M. (2015). Mere observation of body discontinuity affects perceived ownership and vicarious agency over a virtual hand. Exp. Brain Res. 233, 1247-1259.

Todd, J., and Dewhurst, K. (1955). The double: its psychopathology and psycho-physiology. J Nerv Ment Dis 122, 47-55.

Tsakiris, M., and Haggard, P. (2005). The rubber hand illusion revisited: visuotactile integration and self-attribution. J. Exp. Psychol. Hum. Percept. Perform. 31, 80-91.

Tsakiris, M., Hesse, M. D., Boy, C., Haggard, P., and Fink, G. R. (2007). Neural signatures of body ownership: a sensory network for bodily self-consciousness. Cereb. Cortex 17, 2235-44.

Tsakiris, M., Prabhu, G., and Haggard, P. (2006). Having a body versus moving your body: How agency structures bodyownership. Conscious. Cogn. 15, 423-32.

Vallar, G., and Ronchi, R. (2009). Somatoparaphrenia: a body delusion. A review of the neuropsychological literature. Exp. Brain Res. 192, 533-51.

van Beers, R. J., Sittig,A. C., and Gon, J. J. D.Van Der (1999). Integration of Proprioceptive and Visual Position-Information: An Experimentally Supported Model. J. Neurophysiol. 81, 13551364.

Vann, S. D., Aggleton, J. P., and Maguire, E. a (2009). What does the retrosplenial cortex do? Nat. Rev. Neurosci. 10, 792802.

Vatakis, A., and Spence, C. (2007). Crossmodal binding: evaluating the "unity assumption" using audiovisual speech stimuli. Percept. Psychophys. 69, 744-756.
Vroomen, J., and Keetels, M. (2010). Perception of intersensory synchrony : A tutorial review. Atten. Percept. Psychophys. 72, 871-884.

Zeller, D., Litvak, V., Friston, K. J., and Classen, J. (2015). Sensory Processing and the Rubber Hand Illusion-An Evoked Potentials Study. J. Cogn. Neurosci. 27, 573-582.

Zopf, R., Harris, J. A., and Williams, M. A. (2011a). The influence of body-ownership cues on tactile sensitivity. Cogn. Neurosci. 2, 147-154.

Zopf, R., Savage, G., and Williams, M. A. (2010). Crossmodal congruency measures of lateral distance effects on the rubber hand illusion. Neuropsychologia 48, 713-25.

Zopf, R., Truong, S., Finkbeiner, M., Friedman, J., and Williams, M. A. (2011b). Viewing and feeling touch modulates hand position for reaching. Neuropsychologia 49, 1287-93. 



\section{CHAPTER 2}

\section{The Building Blocks OF the Full Body OWNership lluusion}

Related publication:

The building blocks of the full body ownership illusion.

Maselli, A., and Slater, $M$.

Front. Hum. Neurosci. 7, 83, 2013 


\section{Abstract}

Previous work has reported that it is not difficult to give people the illusion of ownership over an artificial body, providing a powerful tool for the investigation of the neural and cognitive mechanisms underlying body perception and self consciousness. We present an experimental study that uses immersive virtual reality focused on identifying the perceptual building blocks of this illusion. We systematically manipulated visuotactile and visual sensorimotor contingencies, visual perspective, and the appearance of the virtual body in order to assess their relative role and mutual interaction. Consistent results from subjective reports and physiological measures showed that a first person perspective over a fake humanoid body is essential for eliciting a body ownership illusion. We found that the level of realism of the virtual body, in particular the realism of skin tone, plays a critical role: when high enough, the illusion can be triggered by the sole effect of the spatial overlap between the real and virtual bodies, providing congruent visuoproprioceptive information, with no need for the additional contribution of congruent visuotactile and/or visual sensorimotor cues. Additionally, we found that the processing of incongruent perceptual cues can be modulated by the level of the illusion: when the illusion is strong, incongruent cues are not experienced as incorrect. Participants exposed to asynchronous visuotactile stimulation can experience the ownership illusion and perceive touch as originating from an object seen to contact the virtual body.Analogously, when the level of realism of the virtual body and/or the spatial overlap of the two bodies is not high enough, the contribution of congruent multisensory and/or sensorimotor cues is required for evoking the illusion. On the basis of these results and inspired by findings from neurophysiological recordings in the monkey, we propose a model that accounts for many of the results reported in the literature. 


\section{INTRODUCTION}

The experience of our body results from the complex interplay of various perceptual streams involving vision, touch, proprioception, interoception, motor control, and vestibular sensations. Strong evidence supporting this statement comes from a number of neurological conditions where the sense of the body becomes altered as a consequence of focal brain lesions, limb amputation or deafferentation. Examples of such conditions are somatoparaphrenia and associated anosognosia (Berlucchi and Aglioti, 1997, 2010; Vallar and Ronchi, 2009), phantom limb sensations (Giummarra et al., 2007), and out-of-body experiences (OBEs) (Blanke and Mohr, 2005).

Altered body perceptions analogous to those experienced in lesioned patients, can be temporally induced in healthy subjects in controlled experimental settings, where the delivery of anomalous sensory stimuli is systematically manipulated.These altered perceptual states are usually referred to as bodily illusions and have been extensively used for studying body perception, self-consciousness and their underlying neural correlates. In particular, experimentally controlled bodily illusions allow, to some extent, the isolation of the various components that converge in the holistic experience of our bodies.

In this paper we present a study that investigates the main perceptual components of the full body ownership illusion, a specific type of bodily illusion in which healthy subjects experience an artificial body as if it were their own physical body. This illusion is particularly interesting for the study of self-consciousness as it relies on an altered representation of the entire body (Blanke and Metzinger, 2009; Blanke, 2012) and, as such, it is complementary to the "rubber hand illusion" (RHI) paradigm in the study of body perception.

In the original version of the RHI (Botvinick and Cohen, 1998), participants see a rubber hand located in front of them in a similar posture to their corresponding but hidden real hand. Both rubber and real hands are stroked by the experimenter. When the strokes on the real and rubber hands are delivered synchronously and in the corresponding spatial locations, most participants experience the rubber hand as if it were their own and feel the touch as originating from the rubber hand itself; the same does not happen, or to a much lesser extent, when the stroking is asynchronous, or when there are other inconsistencies (such as spatial) between the stroking on both hands. A large number of studies have adapted and extended this classical set-up providing experimental evidence about the different perceptual components that contribute to the RHI. These include the spatial configuration and appearance of the artificial hand and the delivery of multisensory and/ or sensorimotor stimulation. The spatial configuration of the artificial hand with respect to the real body affects the illusion. For example, the illusion does not occur when anatomical constraints are violated: when the rubber hand is located outside the participant's peripersonal space (e.g., Lloyd, 2007), when it is in impossible postures (e.g., Ehrsson et al., 2004; Tsakiris and Haggard, 2005), or when it does not represent the main topological features of a hand (Tsakiris and Haggard, 2005). In terms of perceptual cues, the spatial configuration component corresponds to a stream of visuoproprioceptive information in which the spatial location encoded by vision is compared with the one encoded by proprioception. The body's spatial location encoded by proprioception can be 
altered, so that it does not always coincide with the position of the physical body. This happens in $\mathrm{RHI}$ experiments, where the illusion is typically associated with a mislocalization of the real hand toward the fake hand. Inherent to the RHI paradigm is the delivery of visuotactile stimulation, which has to be synchronous in order to evoke the illusion. However, it has been shown that the illusion is also experienced when visuotactile stimulation is substituted by other modalities of multisensory and/or sensorimotor stimulation, e.g., with sensorimotor contingencies in active or passive movements (Tsakiris et al., 2006; Kalckert and Ehrsson, 2012). This shows that the onset of the illusion is triggered more generally from a stream of congruent multimodal bodily signals (multisensory or sensorimotor) and does not necessarily require visuotactile integration. Finally, visual appearance has been found to play a critical role in the illusion: the fake hand does not need to be necessarily realistic for the illusion to take place, as shown by the numerous reports of ownership experienced over plastic-looking hands or elongated limbs (Schaefer et al., 2007; Kilteni et al., 2012; Preston and Newport, 2012); however, higher levels of realism of the artificial hand, in terms of texture and shape, have been found to enhance the strength of the illusion (Haans et al., 2008). The overall emerging scenario is that the sense of ownership over an external object requires the convergence of two main sources of information: (1) the on-line driving contribution from bottom-up processing of congruent multimodal perceptual cues and (2) the modulating top-down machinery based on a flexible but still robust prior internal body representation that requires that some key anatomical constraints in terms of body shape and visual perspective are preserved (see Graziano and Botvinik, 2002; Makin et al., 2008;Tsakiris, 2010).

Analogous perceptual components have been identified for the illusions of owning a whole artificial body. However, the relative importance of each of these components and their mutual interaction are still a subject of debate. This is partly due to the fact that different experimental procedures have been introduced to extend the RHI for dealing with whole bodies. Two main set-ups have been used and, interestingly, the illusions evoked in the two cases were not the same. Both set-ups use a head-mounted-display (HMD) to occlude the real body from vision and to display the artificial body in stereo. In one case participants saw their own body or that of a mannequin as filmed from $2 \mathrm{~m}$ behind. When exposed to synchronous stroking seen on the back of the visualized body and felt on their own back, participants reported experiencing the sensation of "seeing their own perceived body from a distance" and of perceiving touch as originating from the distant body (Lenggenhager et al., 2007, 2009; Aspell et al., 2009; lonta et al., 2011).

Alternatively, when the stroking was felt on the chest and seen from a first person perspective (i.e., the stick seen as stroking the spatial location corresponding to that of the real chest), the resulting illusion was characterized as a feeling of having one's own center of awareness moved behind the seen body, to the first person perspective position from where the scene was being observed. The true body seen in front was perceived as something like an "empty shell" or a "disowned body" (Ehrsson, 2007; Lenggenhager et al., 2009; Guterstam and Ehrsson, 2012). Although the illusions experienced in these two cases are notably different, they are both referred to as experimentally induced OBEs. In a different experimental set-up, a mannequin (Petkova and Ehrsson, 2008; Petkova et al., 2011) or a virtual body (Slater et al., 2010) was displayed from a first person perspective (1PP), where the artificial body visually substituted the obscured real body. Synchronous stroking of the real and virtual bodies typically induces the illusion of owning the artificial body, which is then perceived as the origin of sensory signals like touch and vision. We refer to this as full body 
ownership illusion.

Two principal open issues emerge from the work so far on full body illusions. These are the role of visual perspective and the role of visuotactile stimulation. The role of visual perspective is a key subject on which two major classes of results are diverging. Reports about the feeling of selfidentification that occurs during experimentally induced OBEs suggest that the sense of ownership can be experienced over an artificial body seen from a third person perspective (3PP) (Lenggenhager et al., 2007, 2009; Aspell et al., 2009; Ionta et al., 2011). However, studies focusing on the full body ownership illusion report that a 3PP over the artificial body does not result in the illusion even when synchronous visuotactile stimulation is delivered (Petkova and Ehrsson, 2008; Slater et al., 2010; Petkova et al., 2011). The difference between these findings could be ascribed to differences in the experimental designs adopted or alternatively to the fact that different kinds of perceptual illusions are involved.The full body ownership illusion experienced in 1PP is described as the feeling of owning an artificial body, which substitutes the real body as the origin of perceptual sensations. (e.g., Petkova and Ehrsson, 2008; Slater et al., 2010). Experimentally induced OBEs have been characterized in different ways according to the specific experimental protocol adopted. Depending on the stroking mode, participants have one of two different experiences: (1) the visual 1PP and tactile sensations coincide in the same body — since there is the sensation of being embodied behind the location of the real body that is seen in front (chest stroking); (2) the visual 1PP and the origin of tactile sensations are divorced, since the visual 1PP is at the real body location, but tactile sensations are attributed to the fake body that is seen in front (back stroking). For a comparative study see Lenggenhager et al. (2009). The latter illusion has been shown to correlate with a recoding of self-location in space as measured by walking responses (Lenggenhager et al., 2007) and mental imagery (Lenggenhager et al., 2009), as well as with a remapping of the spatial representation of visuotactile stimuli as measured by the crossmodal congruency effect (CCE; Aspell et al., 2009). It has been further found to correspond with the specific activation of a number of brain areas such as the temporo-parietal junction (TPJ), the bilateral premotor cortex (PMC) and the medial sensorimotor cortex (lonta et al., 2011; Lenggenhager et al., 2011). Despite this, the possibility that the sense of ownership and touch could be experienced over a body located in the far extrapersonal space is still under discussion. It has been proposed that self-identification during OBEs is not an actual perceptual illusion, but rather a form of self-recognition similar to the self-recognition of oneself in a mirror (Petkova et al., 2011; Ehrsson, 2012). Whether the controversy concerning the role played by visual perspective is grounded in the very nature of the illusion or in uncertainties associated with the experimental set-ups adopted remains to be clearly established.

The second main issue under debate concerns the role of multisensory stimulation as a trigger to elicit the illusion. A conclusion drawn in most studies is that synchronous visuotactile stimulation is necessary to elicit the illusion. This is the case for all the OBE's studies mentioned above. Petkova and Ehrsson (2008) also reported that, together with a 1PP over a humanoid artificial body, synchronous visuotactile stimulation is necessary to trigger the full body ownership illusion (see also, Petkova et al., 2011). Using an immersive virtual reality (IVR) set-up, Slater et al. (2010) came instead to a different conclusion. In their experiment participants wore a tracked HMD, receiving visual sensorimotor contingencies, meaning that the displayed field of view is continuously updated according to head position and orientation. Additionally, a virtual mirror provided synchronous visual feedback of 
head movements (visuomotor correlations). Participants received visuotactile stimulation either synchronously or asynchronously, and in both cases they experienced a strong full body ownership illusion. The fact that participants in this experiment could experience the illusion even when receiving asynchronous visuotactile stimulation seems to contradict the results of Petkova and Ehrsson (2008). However, there are two main differences between the two experimental set-ups. First, visual sensorimotor contingencies were provided in the IVR experiment, while in Petkova et al.'s experiment participants were exposed to a static field of view and were asked not to move their head, which was fixed looking down toward their body. Second, the virtual body in the IVR experiment had a realistic human body appearance, in term of skin texture and clothes, while in the other case the artificial body was a plastic mannequin. Whether these differences could explain the different conclusions drawn from the two experiments regarding the necessity or otherwise of visuotactile stimulation, is still not clear.

In this paper we report a set of experiments that systematically varies factors thought to contribute to the full body ownership illusion. Specifically, we consider the effect of visuotactile correlations and visual (head-based) sensorimotor contingencies, visual perspective and visual appearance. Our main aim was to fit previous results into a common framework and to propose a model that would allow assessment of the relative contributions and reciprocal interactions of such bodily signals to the full body ownership illusion.

\section{Materials and Methods}

\subsection{Overall Design}

Our study consisted of three experiments designed with the aim of exploring and disentangling the relative role of those factors that have been reported to be the building blocks of the full-body illusion: visual perspective, human-like bodily appearance, and visuotactile stimulation. Additionally, we consider the effects visual sensorimotor contingencies provided by head tracking, where the visual field displayed in the HMD is updated according to the tracked movements of the participant's head, in the same way as it would be in physical reality.

In experiment 1 we examined the effects of visuotactile and visual sensorimotor stimulation and their possible interaction. For this experiment we used a between-groups $2 \times 2$ design with two binary factors: visuotactile stimuli could be synchronous (VT) or asynchronous $\left(\perp^{\perp} \mathrm{VT}\right)$; head tracking could be enabled $(\mathrm{HT})$ providing visual sensorimotor contingencies, or disabled ( $\mathrm{HT})$, which is with no head movements and with a fixed field of view, as in the set-up of Petkova and Ehrsson (2008). The design of this first experiment was mainly driven by the attempt to account for the difference in the results reported in Petkova and Ehrsson (2008) and Slater et al. (2010), since one important difference between the two studies concerns visual sensorimotor contingencies that are provided in the latter, but not in the former.

Experiment 2 was designed to explicitly test the hypothesis that visual perspective is a critical factor for the full body ownership illusion. For this we adopted a single-factor design, perspective, which had two levels (1PP vs. 3PP). Head tracking was set to HT and visuotactile to VT. This was 
to examine the effect of perspective in the otherwise most favorable condition for the illusion, i.e., with synchronous visuotactile stimulation and visual sensorimotor contingencies. If 3PP prevents the illusion in this case, we can safely extrapolate that the same will happen for other configurations where at least one of the two stimulations is in an incongruent mode (e.g., $\left.\perp_{\mathrm{VTT}}\right)$, or is not provided (e.g., ᄀHT).

In experiment 3 we examine the impact of the level of realism for the appearance of the artificial body, comparing the effect of seeing a realistic human avatar (HA) vs. a plastic mannequin avatar (MA). The hypothesis was that the level of realism of the visualized body (in terms of skin texture and clothing) can modulate the intensity of the full body ownership illusion. As in the case of experiment 1 , the design of this experiment has been motivated by the second critical difference among the experimental set-ups of Petkova and Ehrsson (2008) and Slater et al. (2010): the visual appearance of the fake bodies. We employed a single-factor design where the bodily appearance varied on two levels (HA vs. MA) with the other factors in the $[1 \mathrm{PP}, \perp \mathrm{VT}$, $\neg \mathrm{HT}]$ configuration. This choice enabled us to disentangle the hypothesized modulation of the illusion intensity by the visual appearance of the artificial body from the effects of multisensory and sensorimotor contingencies.

\section{Table 1 | Combinations of experimental factors adopted for the three experiments.}

\begin{tabular}{llll}
\hline Factors & Experiment 1 & Experiment 2 & Experiment 3 \\
\hline Visuotactile & VT vs. $\perp$ VT & VT & $\perp$ VT \\
Head tracking & $H T$ vs. $\neg H T$ & $H T$ & $\neg H T$ \\
Visual perspective & TPP & $1 P P$ vs. 3PP & $1 P P$ \\
Body appearance & HA & HA & HA vs. MA
\end{tabular}

Table 1 Combinations of experimental factors adopted for the three experiments. All experimental factors vary on two levels: "visuotactile" could be synchronous or asynchronous (VT vs. $\perp_{V T}$ ); "headtracking" could be enabled or disabled, providing or not visual sensorymotor contingencies (HT vs. ᄀHT); "visual per- spective" could be first person or third person perspective (1PP vs. 3PP); and "body appearance" could be either human or mannequin appearance (HA vs. MA).The variables in orange show the levels of the experimental fac- tors, while the ones in black show the fixed configuration adopted for each experiment. There were nine participants in each of the four factorial cells of experiment 1, another nine participants in the 3PP condition of experiment 2, and another nine in the MA condition of experiment 3, resulting in a total of 54 participants. 


\subsection{IMPLEMENTATION}

The factors that were manipulated in the three experiments are summarized in Table Table1:1: visual perspective (1PP vs. 3PP), bodily appearance (HA vs. MA), visuotactile stimulation (VT vs. $\perp$ VT), and visual sensorimotor stimulation from head tracking (HT vs. ᄀHT). Although all the four factors vary on two levels, the three experiments planned for this study involve in total six conditions, as some factor combinations could be used for more than one experiment.

When head tracking was enabled, participants could explore the virtual environment and, looking down, they could see the virtual body when in the 1PP condition. In the $\neg \mathrm{HT}$ conditions the virtual scene was instead static and participants were instructed not to move their head and gaze direction, which was fixed looking down toward the virtual body.

Figures 1A, B (1D, E) show the perspective levels for a female (male) participant. For the case of $3 \mathrm{PP}$, the point of view was shifted horizontally $40 \mathrm{~cm}$ away to the right; head tracking was enabled, so that participants could see the virtual body to their left, in their near peripersonal space (i.e., within reach). Participants in this condition also saw the avatar's head moving as their own while exploring the environment, which provided them with additional visuomotor correlations. Note that since the avatar's head followed the movements of the participant's head, participants never got to see the avatar's face (see Figures 1B, E). The avatars used to test the role of the body visual appearance are shown in Figures $1 \mathrm{~A}, \mathrm{C}(1 \mathrm{D}, \mathrm{F})$ for the case of a female (male) participant.

The apparatus used to implement the visuotactile stimulation is shown in Figure Figure2.2. Touch was delivered via mechanical vibrators attached to a haptic vest, while the seen touch was provided in the form of a ball bouncing on the virtual body along a pre-recorded path. The location of the vibrators on the haptic vest was adjusted for each participant, so as to match the contact points of the ball's pre-recorded trajectory with the chest of the avatar. In the VT condition each vibrator was activated for a short time interval $(20 \mathrm{~ms})$ when the simulator detected a collision of the ball with the avatar mesh in the corresponding point (Spanlang et al., 2010; Pomes et al., 2012). The time delay of the vibrator activation with respect to the virtual collision corresponds to the communication time between the machine running the IVR software and the haptic vest and was negligible (<10 ms). The asynchronous condition was implemented by randomly activating the vibrators when the virtual tapping was visualized, so that the touch and the visual collisions were not correlated.

\subsection{Participants}

A total of 54 naïve subjects were recruited for the study, 9 for each of the six conditions over the three experiments (Table 1). Participants were recruited from the University campus or from a database of persons who had previously agreed to being contacted for participation in VR experiments. All subjects participated in only one experiment - i.e., it was a between-groups design. Experiment 1 tested 36 subjects (18 female, mean age 23.3, SD 5.1). Experiments 2 and 3 each involved 18 subjects (experiment 2: 7 female, mean age 29.4, SD 7.6; experiment 3: 8 female, mean age 25.4, SD 4.6). Note that the [VT, HT] group in experiment 1 has the same experimental 
configuration as the 1PP experimental group in experiment 2. For this reason we could use the same experimental group independently for the two experiments. The same applies for the $[\perp \mathrm{VT}, \neg \mathrm{HT}]$ group in experiment 1 and the HA group in experiment 3.
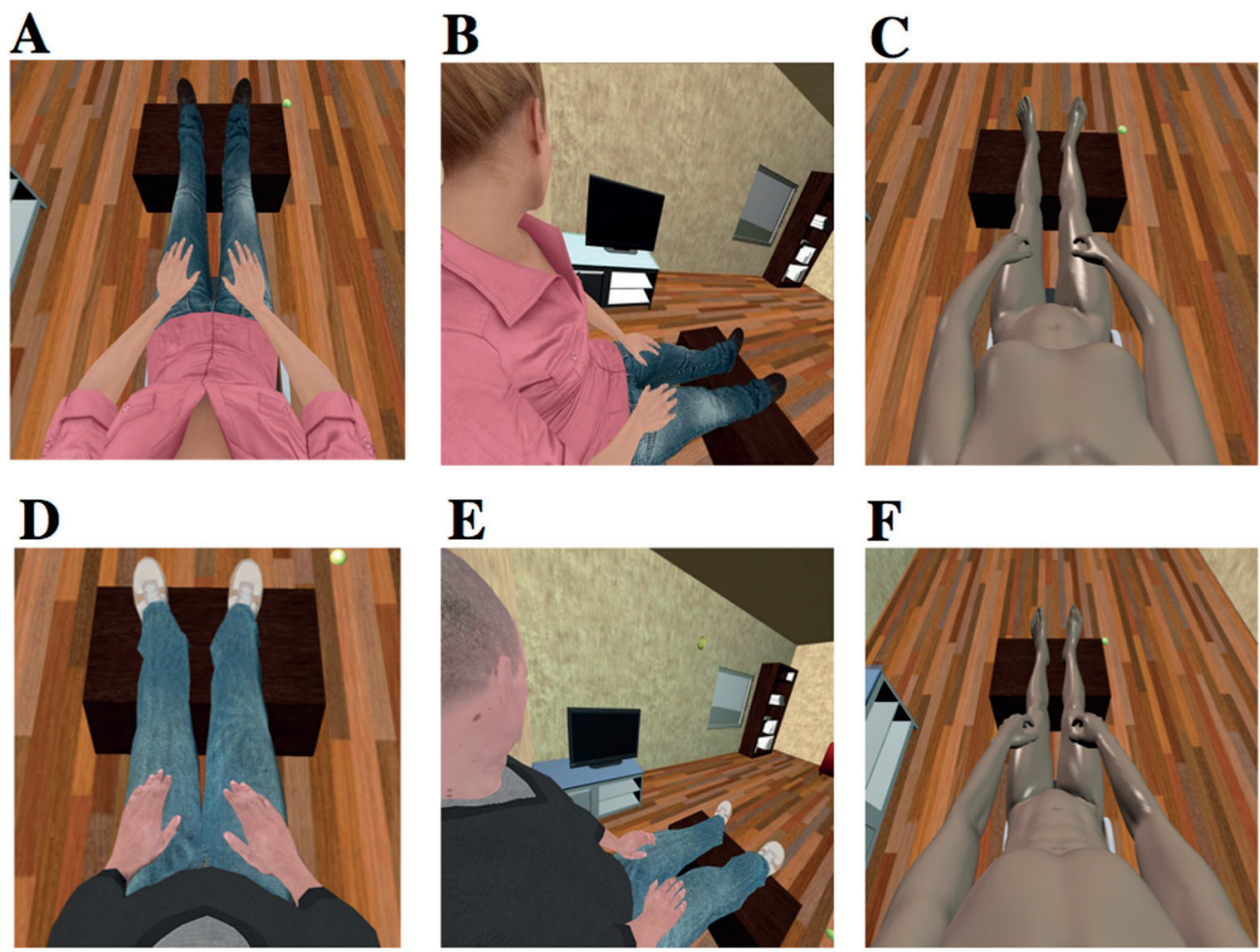

Figure 1. Perspective levels and body appearances levels used for the different conditions of the experiments. Gender-matched avatars were assigned to each participant. $[A, B(D, E)]$ Show the two levels of the factor "perspective" for a female (male) participant: in the 1PP conditions (A,D,C,F) when looking down participants could see a virtual body co-located with their own physical body, while in the 3PP conditions $(B, E)$ participants saw a virtual body in their near extrapersonal space when looking to the left. The two levels of the factor "bodily appearance" are shown in $[A, C(D, F)]$ for a female (male) participant: in the HA conditions participants had a co-located virtual body with a realistic human appearance $(A, D)$, while in the MA condition a co-located virtual body resembling a plastic mannequin (C,F).

The study was performed according to institutional ethics and national standards for the protection of human participants, and it was approved by the Comité Ético de Investigación of the University of Barcelona. All participants signed an informed consent form and filled in a pre-questionnaire to 
collect demographic information before taking part in the experiment. They were then assigned arbitrarily to one of the six conditions and were paid $€ 10$ after the experiment was concluded. In the pre-questionnaire all participants were asked 'Have you ever experience 'virtual reality' before?", with possible responses on a scale from 1 (no experience) to 7 (extensive experience). The response was on average low (median value 1), with only 6 participants out of 54 scoring 4 or higher values.
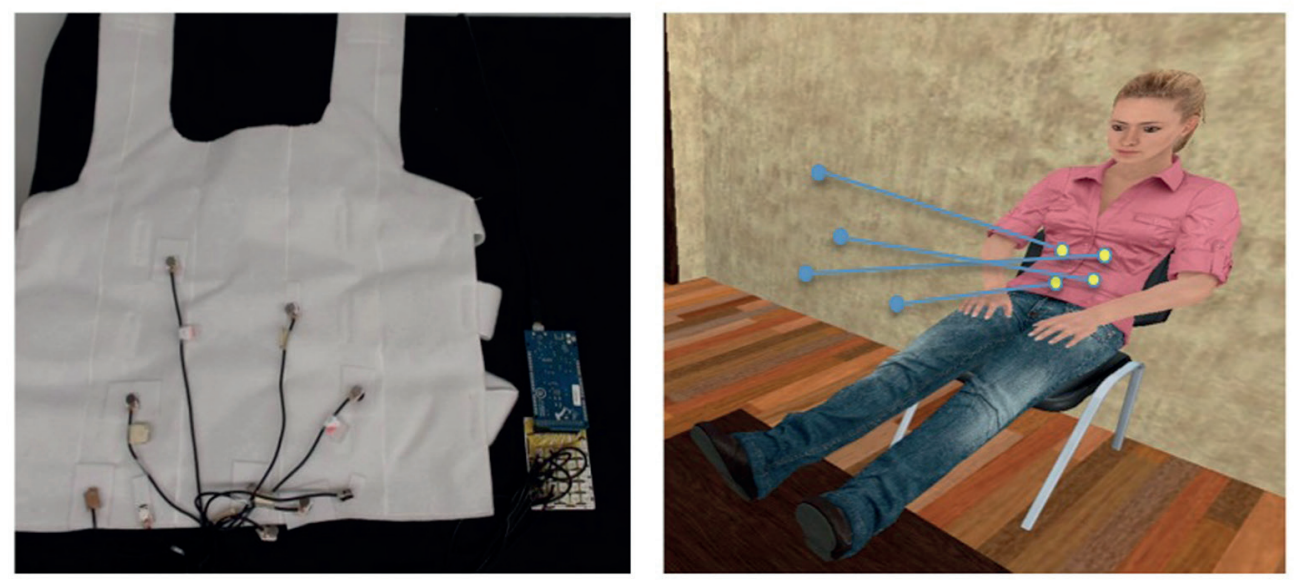

Figure 2.The vibrotactile device used in the experiments. The left panel shows the haptic vest used to deliver touch sensations: vibrators were located on the vest, mapping the location of the contact points of the ball's pre-recorded path with the avatar's chest. The right panel shows the paths followed by the virtual ball (blue lines) and its contact points with the avatar chest (yellow dots). In synchronous conditions the vibrators array was synchronized with the pre-recorded path, so that each vibrator was activated when the ball reached the corresponding path-chest contact point.

\subsection{Experimental Procedure}

Participants were instructed to sit comfortably on a chair with their legs resting on a footstool. They entered the virtual environment using a wide field-of-view head-tracked, HMD. We used a NVIS nVisor SX111 HMD with dual SXGA displays of $76^{\circ} \mathrm{H} \times 64^{\circ} \mathrm{V}$ degrees field of view per eye, corresponding to a global field-of-view of $111^{\circ}$ horizontal and $60^{\circ}$ vertical, with a resolution of $1280 \times 1024$ per eye displayed at $60 \mathrm{~Hz}$. Head tracking was performed by a 6-DOF Intersense IS900 device. Depending on the gender and the condition assigned, each participant was exposed to one of the scenes displayed in stereo within the HMD, shown in Figure Figure1.1. Participants in every condition were instructed to not move their body from the neck down for the whole duration of the experiment. Participants in HT conditions were explicitly asked to turn their head and look around the environment, while those in $\neg \mathrm{HT}$ conditions were asked not to move the head and not to gaze around. 
After an adaptation period of about $30 \mathrm{~s}$, a yellow ball appeared in the scene and moved toward the virtual body. When it struck the body it activated the vibrators on the haptic vest (in synchronous or asynchronous mode according to the condition). Then it bounced off the body moving away again. This tapping phase lasted for an interval of $2 \mathrm{~min}$. In this phase participants were instructed to pay attention to the virtual body and to the tapping ball.At the end of the tapping phase the ball disappeared and the vibrators stopped. Participants were then asked to focus their attention on the virtual body, and after about $30 \mathrm{~s}$ they were exposed to a disturbing event: the lower legs slowly moved away from the rest of the body for $10 \mathrm{~s}$, covering a distance of about $1 \mathrm{~m}$ in the virtual room, and after that they returned to the original position to recompose the whole body (see Figure 3 ). The choice of this particular kind of disturbing event instead of a more classic threat to the virtual body is based on two main motivations: first, we wanted to avoid any kind of threat (even if virtual) directed to the spatial location of the real body; second, we looked for an event that explicitly breaks the integrity of the whole body.
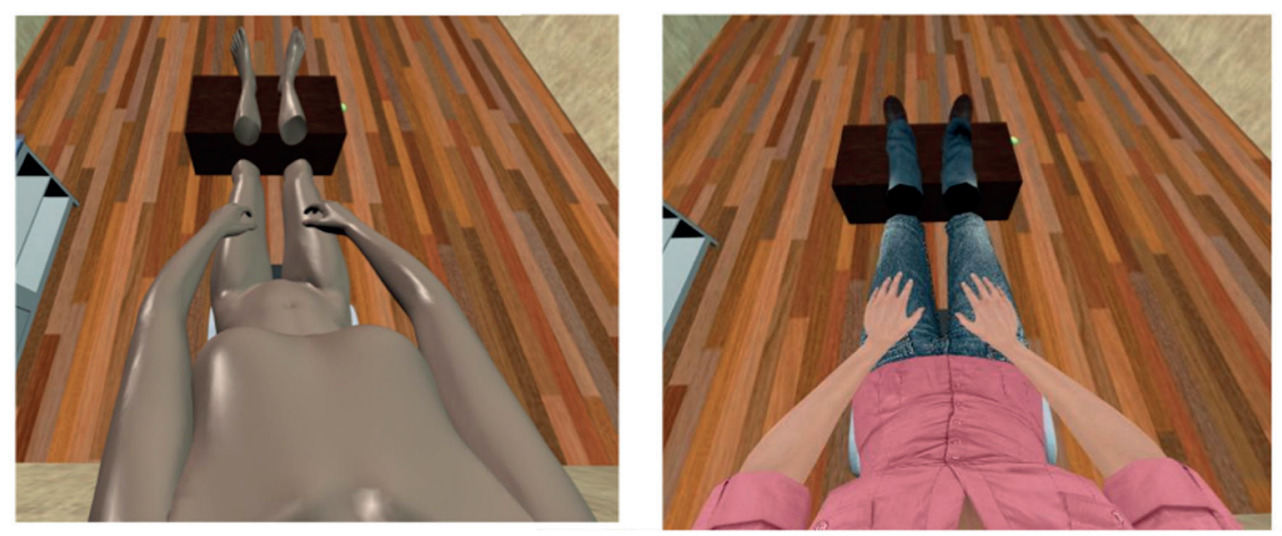

Figure 3. Snapshots of the legs separation event are shown for the mannequin and human appearance modes in the case of a female participants. The legs separation event lasted $10 \mathrm{~s}$ during which the legs moved away from the body reaching a maximum distance of $1 \mathrm{~m}$. The legs returned to their original position immediately after the 10 s, recomposing a whole body.

\subsection{RESPONSE VARIABLES}

\subsubsection{QuestionnAire}

For all the three experiments we used a 7-item questionnaire designed to assess the level and quality of the illusion experienced by the participants. Each question was scored by participants on a given scale from 0 (not at all) to 7 (very much). The choice of an even number of points on the Likert scale 
was meant to force scoring either on the agreement or on the disagreement side. Having four points on each side further allowed for various degrees of agreement/disagreement, so as to detect differences in the intensity of the experienced sensations (Cohen et al., 2011).

The questionnaire is shown in Table 2. The items were formulated by adapting questions from previous experiments on full body illusions (Lenggenhager et al., 2007; Petkova and Ehrsson, 2008; Slater et al., 2010). The main critical item is the one tagged as mybody, which relates directly to the feeling of owning the virtual body. The clothing item was also meant to test the feeling of ownership following Slater et al. (2010) where it was found that the body ownership illusion was highly correlated with the illusion of wearing the same clothes as the virtual character. The items stress and discomfort were formulated to detect the subjective response to the disturbing event. These two apparently similar statements were meant to distinguish the feeling of weirdness (something weird is happening to my body, but it is not stressful) from the feeling of stress that is a state of anxiety. The catch item tested the desire of participants to move and act in the virtual environment. The touch item was meant to test the appropriate implementation of the visuotactile stimulation: the proper functioning the vibrotactile vest and its integration in the virtual environment had to be demonstrated by having low/high scores to the touch item in the asynchronous/synchronous modalities. Finally, the twobodies item was a control question.

Table 2. Questionnaire

\begin{tabular}{lll}
\hline Variable name & Item & Statement category \\
\hline Mybody & I felt that the body I saw was my body & Ownership \\
Clothing & I felt that I was wearing different clothing to my real clothing & Ownership \\
Stress & I was stressed when I saw the legs coming apart & Psychophysical reaction \\
Discomfort & I felt physical discomfort when the legs were coming apart & Psychophysical reaction \\
Catch & I wanted to try to catch the yellow ball & Interaction in VR \\
Touch & It seemed as though the touch I felt was caused by the yellow ball touching my body & Implementation \\
Twobodies & I felt that I had two bodies & Control
\end{tabular}

\subsubsection{Heart Rate Deceleration}

We monitored the physiological behavior of the participants by recording their electrocardiogram (ECG) throughout the whole duration of the experiment. This was used to obtain a measure for the heart rate deceleration (HRD) in various stages of the experiment. HRD has been previously shown to be a response variable that significantly correlates with states of stress and anxiety induced by sudden unpleasant stimuli (Bradley et al., 2001; Cacioppo et al., 2007), and has been successfully used in previous studies as a physiological correlate of the full body ownership illusion (Slater et al., 2010) and other perceptual illusions (e.g., Tajadura-Jiménez et al., 2012). In our study, instead of using a sudden threat, we have adopted a threat to the integrity of the body that is extended in time, i.e., the legs separating from the rest of the virtual body, in slow motion, over a period of 10 s. For this reason it is not possible to identify the precise moment at which participants noticed this event. To account for this, we introduced a new procedural definition for the HRD. 
The ECG signal was sampled at $256 \mathrm{~Hz}$ using the g.tec portable bio-signal acquisition device g.MOBllab ${ }^{1}$. ECG signals were processed to extract the heart rate (HR) by first applying the automatic search for QSR complex implemented in the gtec biosignal analysis software g.BSanalyze ${ }^{2}$, and then by visually inspecting the search results to correct for possible missing or false identifications. The HRD response to the legs separation, HRDlegs, was calculated within the period of $6 \mathrm{~s}$ immediately after the legs started to move away from the rest of the virtual body, by looking for the first sustained deceleration event occurring in this time window (i.e., the earliest time interval in which the HR signal was monotonically decreasing). In order to have a reliable detection of sustained deceleration associated to the unpleasant event, we imposed two constraints: (1) the extent of the time interval of monotonic deceleration had to be larger than $1.5 \mathrm{~s}$, so as to ensure that this was a sustained deceleration; (2) the starting point of the sustained deceleration should be at most $2 \mathrm{~s}$ away from the starting edge of the $6 \mathrm{~s}$ period. The latter constraint was meant to account for the possibility that the participants did not immediately detect the onset of the unpleasant event. Once we identified the deceleration event in the HR signal, we calculated the HRD as: $\Delta H R / \Delta t$, with $\Delta H R$ $=H R\left(t_{\max }\right)-H R\left(t_{\min }\right)$ and $\Delta t=t_{\min }-t_{\max }$. Here tmin and tmax denote the location in time of the minimum and maximum, respectively. For those cases in which the HR signal was monotonically increasing within the whole $6 \mathrm{~s}$ window, we have used an analogous procedure to obtain negative HRD values, describing in fact a heart rate acceleration.

As the HRD measurements are expected to be subject-dependent, we have further estimated a measure for the HRD baseline of each participant. In order to obtain a representative baseline value, we have applied the same procedure described above for measuring HRD from single sustained deceleration event, in 10 different time windows (each of $6 \mathrm{~s}$ ) within the $20 \mathrm{~s}$ rest period preceding the legs separation. The baseline value, HRDbase was defined as the mean of these 10 HRD measures.

\section{Results}

\subsection{Statistical Methods}

Since the responses to the questionnaires are ordinal and not interval scaled, we used non-parametric tests for the questionnaire analysis. Data from experiment 1 were analyzed with the Friedman test for non-parametric analysis of $2 \times 2$ designs for testing the effect of each factor, while taking into account the other as a blocking factor. Note that the Friedman test does not allow detection of interaction effects. When comparing two samples, as in experiments 2 and 3 and in some post analysis of experiment 1 , we used the Mann-Whitney $U$ test.

The HRD data were inspected in order to look for changes induced by the legs separation event, one that obviously affected the integrity of the body, with respect to the baseline. We performed analysis of variance (ANOVA) comparing the change in HRD induced by the threat and measured as $\triangle H R D=H R D_{\text {legs }}-H R D_{\text {base }}$, between the experimental conditions of each experiment. Finally, we bring various results together in one simple path analysis model that helps understand the results of the experiments.

1 http://www.gtec.at/Products/Hardware-and-Accessories/g.MOBllab-Specs-Features

2 http://www.gtec.at/Products/Software/g.BSanalyze-Specs-Features 
All analysis was carried out using the Stata 12 statistical package ${ }^{3}$

\subsection{Experiment 1: Multimodal Contingencies}

\subsubsection{QuestionNAIRE}

Experiment 1 was a $2 \times 2$ design with factors visuotactile $\left(\mathrm{VT}\right.$ vs. $\left.\perp^{\mathrm{V} T}\right)$ ) and head tracking $(\mathrm{HT}$ vs. $\neg \mathrm{HT}$ ). Figure 4 shows the boxplots comparing the distributions of scores in the four conditions for relevant items. It is evident that there is no significant difference between the four conditions with respect to the sensation of body ownership (item mybody). The Friedman test applied to the mybody item confirmed that there is no significant difference with respect to both the visuotactile and head tracking factors. Median scores were at least 4 in all the four groups, suggesting that participants experienced some illusion of body ownership over the virtual body whatever the visuotactile and head tracking modes. Responses to the clothing item also received high scores in all the four conditions (all medians at least 4).

The stress and discomfort items had median values below 4 and no significant difference was found between the four conditions. Nevertheless, verbal reports revealed that most participants experienced a strange sensation while seeing the virtual legs separating, a fact often corroborated by spontaneous exclamations and verbalizations during and after the legs separation.

Scores to the touch item were significantly higher for the VT conditions than for the $\perp_{\mathrm{VT}}$, for both the HT ( $p=0.009$, Mann-Whitney; average ranks: 6.28 for $\perp \mathrm{VT}, 12.72$ for $V T)$ and $\neg \mathrm{HT}(\mathrm{p}=0.001$, Mann-Whitney; average ranks: 49 for $\perp$ VT, 122 for VT) groups, showing that our implementation of the visuotactile stimulation via automatic mechanical vibrators was effective. On the other hand participants had significantly greater scores in the HT compared to the $\neg \mathrm{HT}$ mode, in the $\perp$ VT mode ( $p=0.048$, Mann-Whitney; average ranks: 7.06 for $\neg \mathrm{HT}, 11.94$ for $\mathrm{HT}$ ), while no significant difference between the HT levels is present in the VT mode group. The Friedman test applied to the touch item detected significant differences with respect to both the VT factor $(p<0.0005)$ and HT factor $(p=0.042)$. It is noticeable that the asynchronous visuotactile stimulation was clearly perceived as wrong (median score is 1 ) only in the $\left[\perp^{-V T}, \neg H T\right]$ condition, while in the $\left[\perp^{-V T}, H T\right]$ condition the same asynchronous stimulation till had a median score of 4 . Hence some participants still perceived this as a touch sensation congruent with the collision of the virtual ball with the body - as indicated from their reports and scores that reached as high as 6 .

3 http://www.stata.com/stata12/ 

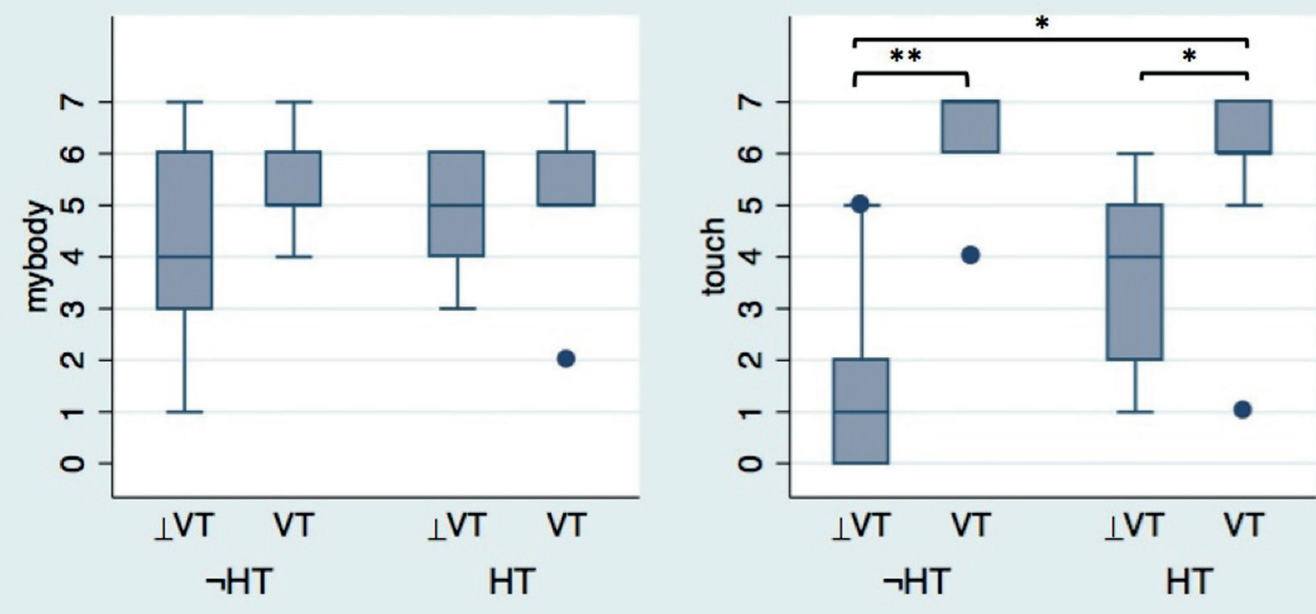

Figure 4. Questionnaire data from experiment 1, in which we investigate the role of viusotactile and visual sensorimotor contingencies. No difference was found in the mybody item showing that the illusion was not affected by either of the two multisensory modalities (when a 1PP is provided over a realistic virtual body). Significant differences were found for the touch item with respect to the visuotactile mode, for both levels of the head tracking factor. This showed that the vibrotactile device used to deliver touch sensations was effective. $* p<0.05, * * p<0.01$, *** $p<0.001$ from Mann-Whitney test.

Scores to the catch item were significantly greater in the HT condition than in the $\neg \mathrm{HT}$ for the $\perp_{\mathrm{VT}}$ group ( $P=0.014$, Mann-Whitney; average ranks: 6.5 for $\neg \mathrm{HT}, 12.5$ for HT). No significant difference was found for the VT group. Analogously, a significant difference with respect to the visuotactile factor was found for the $\neg \mathrm{HT}$ group $(p=0.015$, Mann-Whitney; average ranks: 6.5 for $\perp \mathrm{VT}, 12.5$ for VT) but not for the HT one. The Friedman test detected significant differences between the scores to the catch item with respect to the head tracking factor $(0.005)$, but not for the visuotactile one. It is interesting to note that the median score was much smaller only in the $\left[{ }^{\perp} \mathrm{VT}, \neg \mathrm{HT}\right]$ condition. For the other three groups, when congruent multisensory and/or visual sensorimotor correlations were provided (VT or HT or both), the scores tended to be quite high with medians at least 4. This showed that the desire to move and act in the virtual environment was mainly driven by multisensory/ sensorimotor correlations and did not depend on the sense of ownership over the virtual body alone.

Since the level of ownership turned out to be independent of two experimental factors, we can further examine whether there are some undetected differences in the scoring to the mybody item by combining samples. We first compared the two visuotactile levels irrespectively of the head tracking mode and vice versa (18 subjects per group). In both cases the Mann-Whitney test found no significant difference. The same procedure was applied to the other questionnaire items for which no dependence on either VT and HT was found (all but touch and catch). This confirmed previous 
findings, i.e., that the feeling of ownership over the virtual body did not depend on either the visuotactile and the head tracking modes signals.

\subsubsection{Heart Rate Deceleration}

Two-Way ANOVA of $\triangle H R D$ showed that neither of the two factors, nor their interaction, was significant in modulating the HRD response when threatening the integrity of the body [VT: $\mathrm{F}_{(1,32)}=$ $0.40, \mathrm{p}=0.53 ; \mathrm{HT}: \mathrm{F}_{(1,32)}=1.32, \mathrm{p}=0.26$; interaction: $\left.\mathrm{F}_{(1,32)}=1.80, \mathrm{p}=0.19\right]$. We have further checked specifically for the effect of the visuotactile factor, collapsing together the two headtracking levels. The same was done for testing further the effect of the headtracking factor, grouping together the visuotactile levels. In both cases no significant differences were found $\left[V T: F_{(1,34)}=0.39, p=0.54 ; H T\right.$ : $\left.F_{(1,34)}=1.31, p=0.26\right]$.

\subsection{Experiment 2: Perspective}

\subsubsection{Questionnaire}

Figure 5 shows the boxplot comparing scores to the mybody items from 1PP and the 3PP groups. From the figure one can see a noticeable effect of visual perspective. Comparing the two groups, [1PP,VT, HT] vs. [3PP,VT, HT] with 9 participants each, a Mann-Whitney test returned a significant difference $(p=0.004$; average ranks:5.89 for 3PP,13.11 for 1PP). For all the other items no significant differences were found.

Given the results from experiment 1, i.e., visuotactile and head tracking modes have no effect on the ownership illusion, we combined the groups across these levels in order to increase the power of the test. The Mann-Whitney test comparing the 3PP group ( 9 subjects) with that of 1PP with both visuotactile and head tracking levels merged (36 subjects), resulted in significant differences in the perspective levels for both the item mybody, now with greater significance $(p=0.0007$; average ranks: 10 for 3PP, 26.25 for 1PP), and clothing ( $P=0.024$; average ranks: 14.28 for 3PP, 25.18 for 1PP).

\subsubsection{Heart Rate Deceleration}

One-Way ANOVA of $\triangle H R D$ resulted in no significant effect of the perspective factor on the HRD response when threatening the integrity of the body $\left[F_{(1,16)}=1.21, p=0.26\right]$. No difference was found when further comparing the $3 \mathrm{PP}$ group with the $1 \mathrm{PP}_{36}$ group $\left[\mathrm{F}_{(1,16)}=0.10, \mathrm{p}=0.75\right]$ 


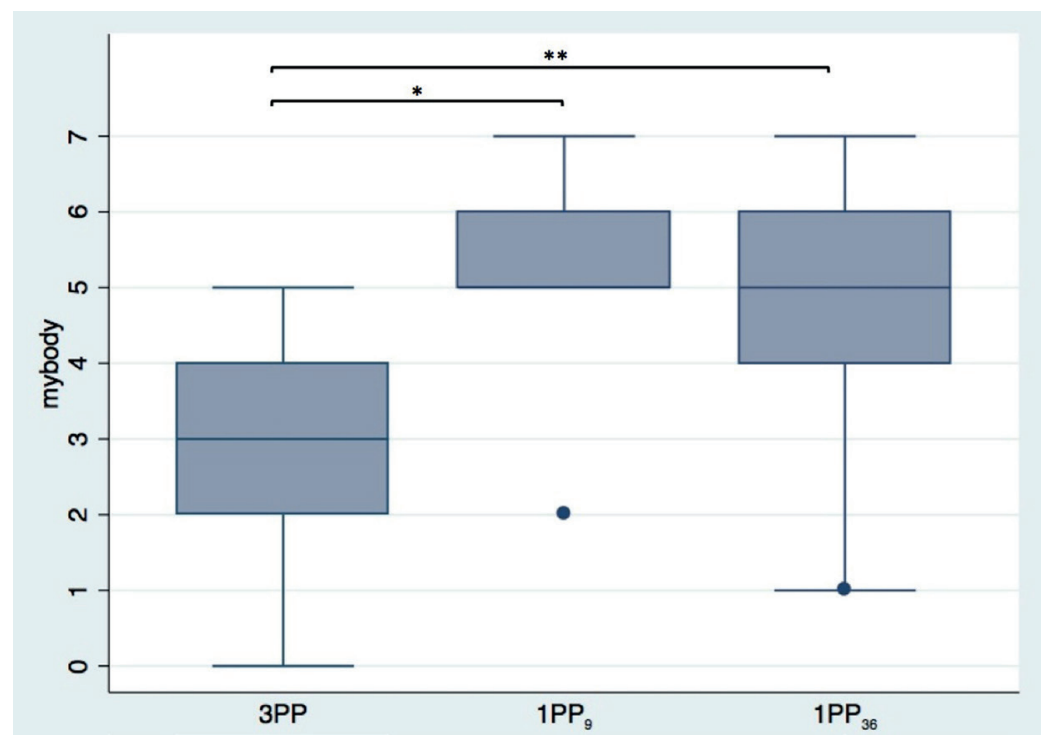

Figure 5. Questionnaire data from experiment 2, in which we compare groups with different perspective levels. $3 P P$ (9 subjects), $1 \mathrm{PP}_{9}$ (9 subjects) and $1 \mathrm{PP}_{36}$ (36 subjects, where all 1PP conditions were grouped together irrespectively of the visuotactile and head tracking modes). The significant difference in the mybody item showed that 1PP is an essential condition for the full body ownership illusion.

** $p<0.01$, *** $p<0.001$ from Mann-Whitney test.

\subsection{EXPeriment 3: Bodily Appearance}

\subsubsection{Questionnaire}

Figure 6 shows the boxplots comparing the scores to the item mybody for the HA and MA groups. No significant differences were found between the two groups in the $[1 \mathrm{PP}, \perp \mathrm{VT}, \neg \mathrm{HT}]$ configuration (9 subjects per group; $\mathrm{HA}_{9}$ vs. MA). However, since there was no dependence of the ownership illusion on the visuotactile and head tracking factors (results from experiment 1), we could group the levels of these two factors. Doing so we obtained a larger sample of 36 subjects for the $\mathrm{HA}\left(\mathrm{HA}_{36}\right)$ condition to be compared with the MA group ( 9 subjects). In this case we found significant difference for both the items mybody $(p=0.003$; average ranks: 11.44 for HA, 25.89 for HA) and clothing $(p=$ 0.011; average ranks: 13.17 for HA, 25.46 for HA). This suggests that a realistic body appearance significantly enhances the ownership illusion. It is important here to note that despite the differences found in the two visual appearance groups and despite the asynchronous visuotactile stimulation, the sense of ownership was present to some extent even when the virtual body had a mannequin appearance, as shown from the fact that participants in the MA group had a median score of 3 on the mybody item. 


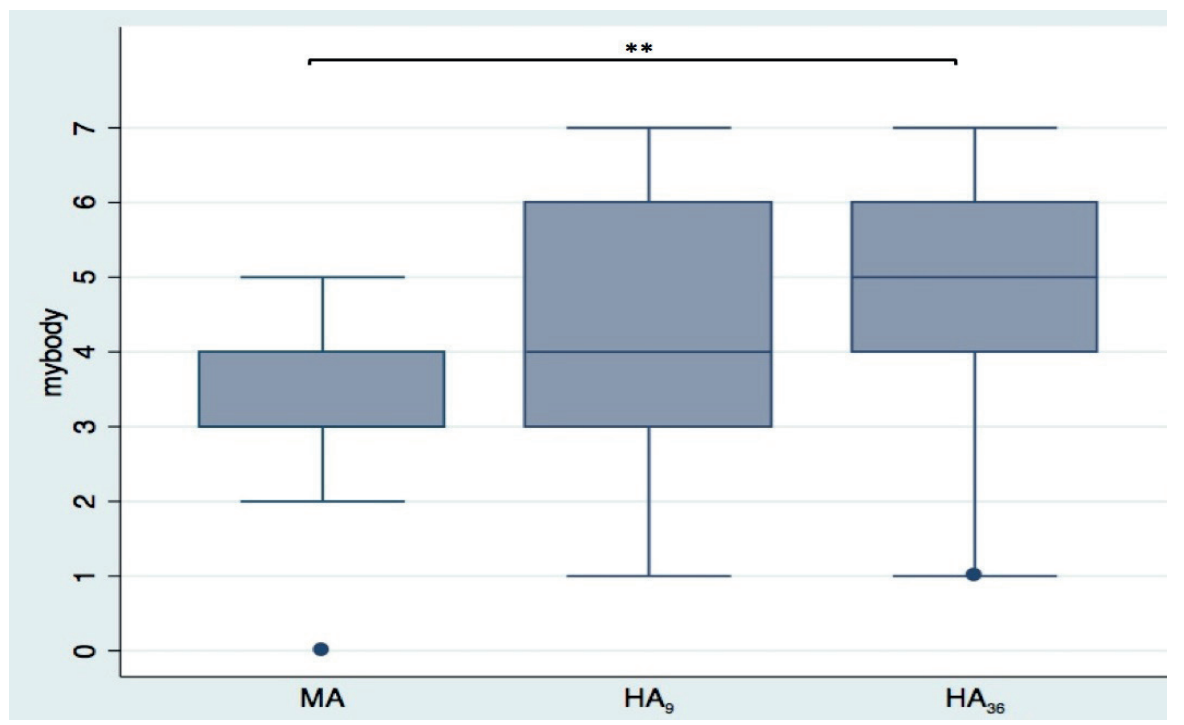

Figure 6. Questionnaire data from experiment 3, in which we compared two groups that had different virtual bodies. Mannequin appearance: MA (9 subjects); human appearance: $H A_{9}(9$ subjects) and $H A_{36}$ (36 subjects, where all the human appearance conditions in the 1PP were grouped together irrespectively of the visuotactile and head tracking mode). Significant differences were found when comparing the MA group with the $\mathrm{HA}_{36}$ group. This showed that a realistic body appearance significantly enhances the illusion experience. ** $p<0.01$ from Mann-Whitney test.

\subsubsection{Heart Rate Deceleration}

One-Way ANOVA showed no significant difference in $\triangle H R D$ between the two body appearance groups $\left[F_{(1,16)}=1.24, P=28\right]$. No difference was found when further comparing the MA group with the $\mathrm{HA}_{36}$ group $\left[\mathrm{F}_{(1,16)}=0.60, \mathrm{p}=0.44\right]$.

\subsection{Path Analysis}

It is not too surprising that we found no apparent effect on the HRD of the legs separation threat to the body, since there was no differential effect on body ownership of most of the factor levels. We would expect that only in conditions of a high body ownership illusion would the threat to the integrity of the body cause the type of abhorrent response that might be reflected in $\mathrm{HRD}_{\text {legs }}$. We found a strong impact on ownership only for the manipulation that involved perspective (experiment 2). Hence in this case we would expect an impact on $\mathrm{HRD}_{\text {legs }}$. However, the situation is not straightforward since the HRD during the event would be likely to be associated with the baseline HRD but also and separately be associated with the level of ownership, which itself is impacted by the manipulation in perspective (1PP or 3PP). For this reason we used path analysis to 
further explore the possibility that $H R D_{\text {legs }}$ is indirectly affected by perspective through the feeling of ownership, a possibility that is not testable using simple analysis of variance. We propose the path model in Figure 7, in which perspective directly affects ownership, ownership affects $H_{R D}$ legs, and $H R D_{\text {legs }}$ further depends on $H R D_{\text {base }}$. The latter path takes into account intrinsic HRD differences between individuals. We used the scores from the questionnaire item mybody as a quantitative estimate of ownership. Note that here we treat mybody as an interval scaled variable; even if this is not formally justified, it is an approximation usually adopted as it provides useful exploratory tool.

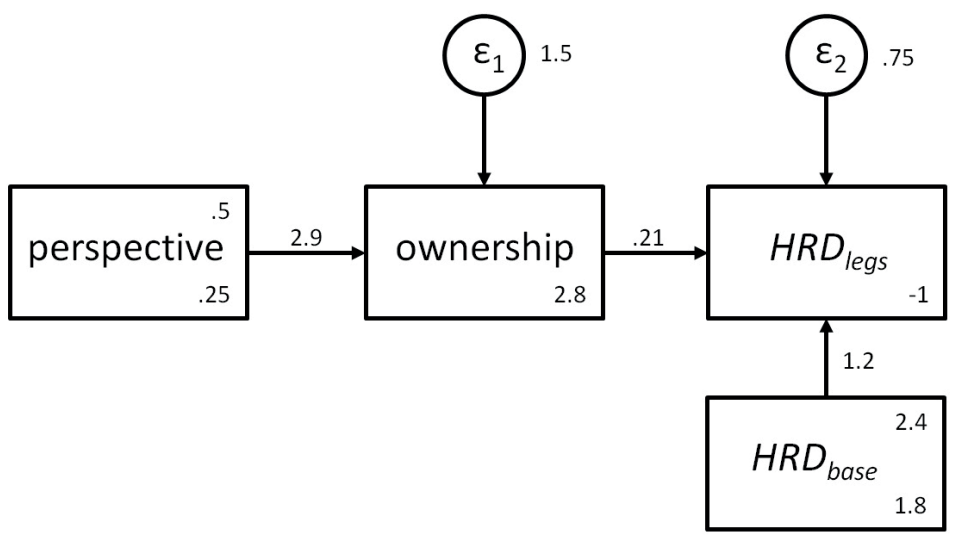

Figure 7. Path analysis model that fits data from experiment 2. The boxes represent the variables: perspective can be 0 or 1 for 3PP and 1PP, respectively; ownership is the response to the questionnaire item mybody; $H R D_{\text {base }}$ is the heart rate deceleration baseline, and $H R D_{\text {legs }}$ is the heart rate deceleration measured right after the legs start separating. The circles represent random error terms.

We used the structural equation modeling software of Stata 12 to estimate the coefficients and the corresponding significant levels, using the asymptotic distribution free option, since we had no reason to suppose that the variables involved follow a multivariate normal distribution.

The model proposed provides a very good fit to the data. A test of the goodness of fit of the model against the saturated model showed a good fit, with $\chi^{2}(2)=2.04, p>0.36$. All estimates are summarized in Table 3. This result shows how the feeling of ownership is strongly affected by perspective as already discussed.Additionally it shows that, even though there is there is not a direct significant impact of perspective on HRD, the response to the threat in terms of HRD increases with ownership. Also, as expected, the HRD response to the threat is highly correlated with $H R D_{\text {base' }}$, reflecting the intersubject HRD variability.

The factor perspective then has an effect on HRD mediated through the subjective level of ownership as measured by the questionnaire variable mybody. 
Table 3. Coefficients, standard errors, and p-values estimates from path analysis applied to the model in Figure 6, using data from experiment 2.

\begin{tabular}{lclr}
\hline Variable & Coefficients & Standard errors & $P$ \\
\hline OWNERSHIP & & & \\
Perspective & 2.89 & 0.60 & $<0.001$ \\
Intercept & 2.79 & 0.51 & $<0.001$ \\
HRD & & & \\
Ownegs & 0.21 & 0.70 & 0.003 \\
HRD & 0.82 & 0.60 & $<0.001$ \\
Intercept & -1.03 & 0.27 & $<0.001$
\end{tabular}

\section{Discussion}

Our study contributes several key findings regarding the full body ownership illusion. Three experiments were specifically designed to pinpoint the main perceptual cues that contribute to the full body ownership illusion, to determine their specific role and assess their interaction. Motivated by previous results in the literature, we focused on the following main perceptual cues: visuotactile and visual (head-based) sensorimotor stimulation, visual perspective, and body appearance. The main findings are compatible with the following statements:

- First person perspective is a necessary condition for the full body ownership illusion.

- The full body ownership illusion can result from the sole effect of seeing a realistic virtual body in the same location and posture as the physical body, a configuration that contributes toward the experience of correct visuoproprioceptive cues, with no need for the additional contribution of congruent multisensory and/or head-based sensorimotor cues.

- The appearance of the virtual body and more specifically its level of realism in terms of skin texture and clothes, can influence the strength of the full body ownership illusion.

- Multisensory and/or sensorimotor contingencies can influence the level of the full body ownership illusion, having a reinforcing effect when congruent and a damping effect when incongruent.

- The full body ownership illusion can modulate the way touch is perceived, in that asynchronous visuotactile cues can be consciously perceived as correct, especially when there is first person perspective and a realistic virtual body.

Note that we did not directly explore the effect of visuomotor correlations, as participants could only move the head and were not allow to move the rest of the body. We speculate that congruent visuomotor correlations provide powerful multimodal cues that have a similar - though stronger- effect on the ownership illusion as the multisensory and head-based visual sensorymotor 
contingencies explored in our experiments. This speculation needs to be further tested in a future study.

In the following we discuss the above findings in the context of the relevant literature, highlighting the new findings with respect to previous studies.

\subsection{The Role of Perspective}

In experiment 2 we found clear evidence for 1PP being a critical factor for eliciting the full body ownership illusion. The questionnaire results showed a strong significant difference between participants with 1PP and 3PP, in that only the first group experienced the illusion of ownership with respect to the virtual body. HRD data further support this finding (see section 3.5). Moreover, our experimental set-up showed that 3PP inhibited the illusion even when various form of congruent multisensory and sensorimotor cues were provided. Therefore, as a general result, it could be said that first person perspective over the fake body is a necessary condition for the onset of the full body ownership illusion. Our results bring also further insights on the issue raised in Petkova et al. (2011) as to whether 3PP prevents the illusion because it violates the 1PP as such, or less restrictively, because (as in their experiment) the body to be owned is seen in the far extrapersonal space. In our experimental set-up, the virtual body is seen from 3PP and it is located within the peripersonal space. The finding that the illusion is suppressed in this set-up demonstrates that violation of 1PP over the fake body is sufficient to prevent the full body ownership illusion, even if the body is seen in the peripersonal space.

Our results are consistent with previous findings from studies on both the RHI and the full body ownership illusion. In the RHI paradigm, experiments by different groups have investigated the effect of displacing the position of the artificial hand with respect to the real physical one in order to determine the role of spatial congruency between visual and proprioceptive information. For the sake of the present discussion, the majority of these manipulations could be interestingly reinterpreted in terms of perspective over the body-part to be integrated, having 3PP for those cases in which the fake limb position violates constraints from 1PP, i.e., when the limb is outside the peripersonal space (Armel and Ramachandran, 2003; Lloyd, 2007) or in anatomically impossible configurations, e.g., at $180^{\circ}$ (Ehrsson et al., 2004) and when handendness does not match (Tsakiris and Haggard, 2005). By doing this, the result is that all those cases in which the fake hand position violates constraints from 1PP have been found to work against the illusion, supporting the general conclusion that first person perspective is indeed a critical factor for eliciting the illusion of ownership over an external object. Analogously, studies focused on the full body ownership illusion showed that the illusion could not be induced in various configurations of 3PP, e.g., seeing the body laying in extrapersonal space (Petkova et al., 2011) or seeing the body located to the side in peripersonal space (as in our experiment 2). In both configurations, participants did not experience the illusion despite synchronous visuotactile stimulation and/or visual sensorimotor contingencies being provided.

A second group of studies reported, however, different conclusions on the role of visual perspective 
in ownership illusions. Preston and Newport (2012) presented an experimental setting in which participants experienced an illusion over their own arm filmed from a distance of $2 \mathrm{~m}$ and displayed on a screen (the arm image in the video was also manipulated so to show it elongated). Similarly, a number of studies concern experimentally induced OBEs, in which participants experience selfidentification with a virtual full-body seen from a 3PP (Lenggenhager et al., 2007, 2009; Aspell et al., 2009; lonta et al., 2011). The resulting sense of ownership and touch experienced over a body located in far extrapersonal space is in contradiction with the results reviewed above.

How can these two classes of results be compatible with each other? A possible explanation that has been recently proposed (Petkova et al., 2011; Ehrsson, 2012) is that the self-identification experienced during OBEs is in fact a form of visual self-recognition rather than a somatic ownership illusion, similar to the experience of recognizing oneself in a mirror. An element in favor of this interpretation can be found in the seminal experiment from Lenggenhager et al. (2007), where the same stroking protocol was applied while visualizing either the filmed participant's body or that of a plastic mannequin. Although in both cases a significant difference was found among the synchronous and asynchronous stroking modes, the actual scores of the questionnaire item related to self-identification were lower in the mannequin condition (Lenggenhager et al., 2007supporting online material). This seems to suggest that the self-identification actually occurred only for participant seeing their own filmed body, supporting the "self-recognition" hypothesis. On the other hand, there are several objective measurements that provide evidence for the backstroking induced OBE to be an authentic somatic illusion. Participants experiencing this illusion seem to undergo a remapping of the visuotactile receptive field (RF) (Aspell et al., 2009) and of the self-location in an allocentric spatial reference frame (Lenggenhager et al., 2007, 2009). A selective activation of the TPJ analogous to that occurring in neurological OBEs (lonta et al., 2011), and of bilateral PMC and medial sensorimotor cortex (Lenggenhager et al., 2011) have also been found. We suggest here that the full body ownership and the back-stroking OBE are in fact two different kinds of body illusions, which involve the activation of different neural patterns (see section 4.4). A rigorous comparison of the two illusions requires, however, a dedicated experimental set-up and is beyond the scope of this paper.

A recent study of patients with the somatoparaphrenic delusion of disowning their own left arm (Fotopoulou et al., 2011) provided further insights on the role of visual perspective. It showed that the disownership of the arm in these patients can be suddenly extinguished when the arm is seen from 3PP (in a mirror). Disownership was reinstated, however, a few seconds after switching again to a 1PP over the arm. These results seem to suggest that the sense of ownership requires the activation of a perceptual mechanism specific to 1PP that is impaired in somatoparaphrenic patients. When the perspective is switched to 3PP with the use of mirrors, the visual processing of the own body undergoes a spatial transformation, and it may no longer require the impaired mechanisms responsible for the perceived ownership/disownership. Given the prompt switching between the sense of ownership and disownership, when changing from 1PP to 3PP and vice versa, this mechanism is likely to be purely perceptual and bottom-up. We propose that a plausible candidate for this is provided by consistent visuoproprioceptice cues and by the resulting pattern of neural activation. We discuss this possibility in the following section as well as in section 4.4. 


\subsection{The Role of Multimodal Contingencies}

Results from experiment 1 showed that subjects in an immersive virtual environment may experience a sense of ownership over a highly realistic virtual body seen from a first person perspective, independently of the visuotactile and head-based visual sensorimotor cues received.This is supported from both questionnaire and HRD responses to the event disrupting the integrity of the whole virtual body; no significant differences between the four groups were found in either measurement. This suggests that, when the body is not moving, the effect of congruent visuoproprioceptive cues alone, as provided by having a high degree of spatial overlap between the physical body and the realistic virtual body, is a sufficient condition for inducing a full body ownership illusion. Since the ownership illusion occurs when there is congruent visuoproprioceptive feedback, for a static body, then it should be all the more powerful when there is additionally congruent visuomotor feedback — so that the virtual body moves synchronously with, and spatially matches, real body movements. Note that the requirement for a high degree of overlap between the physical and virtual bodies is a stronger constraint than 1PP by itself, so that it is possible to have 1PP and no congruent visuoproprioceptive cues at the same time. For example, one could have 1PP over a virtual body with a plausible body posture that is nevertheless different from the posture of the obscured physical body, or 1PP over a human-like body that have different size and/or body proportions with respect to the real body. While 1PP seems to be a necessary condition for experiencing an ownership illusion, other work seems to suggest that the exact position and posture of the real and virtual bodies (body parts) do not need to coincide in space (e.g., de la Peña et al., 2010; Petkova et al., 2011). Somatic illusions can be experienced in such configurations if additional congruent multisensory and/or sensorimotor cues are provided, and in these cases proprioception can be altered and shifted toward the virtual body, as shown by measurements of the proprioceptive drift in the RHI (e.g., Botvinick and Cohen, 1998) and OBEs (e.g., Lenggenhager et al., 2007), as well as reports of changes in the perceived body posture (de la Peña et al., 2010). With our experiment we have shown that when there is a high degree of overlap between the real and virtual body, the illusion can be experienced with no need for additional congruent multisensory and/or sensorimotor cues.

Our results further show that the illusion arising from having a high degree of spatial overlap between the virtual and physical bodies can be sustained, even when asynchronous visuotactile stimulation is delivered to the participants and in the absence of any other form of congruent multimodal stimulation. As a further interesting outcome, we found that having or not visual sensoriomotor contingencies from head tracking affected the way participants perceived touch; in the group that received visual sensorimotor contingencies from head tracking throughout the experiment, the asynchronous visuotactile stimulation was not perceived as completely wrong, while when head movements were not allowed and the field of view was static the same visuotactile stimulation was clearly reported to be wrong. This is an important finding that clearly indicates that there is an interaction effect in the way these multimodal stimulations are processed, and that the way touch is consciously perceived can be modulated by the processing of other perceptual cues, possibly via the onset of the illusion.

Altogether, our results present various novel insights with respect to previous studies. In fact, it 
has been extensively reported that the processing of congruent visuotactile and/or sensorimotor stimulation is a necessary condition for the experimental elicitation of bodily illusion in healthy subjects. This is the case for most of the reported experiences of extracorporeal object assimilation (Botvinick and Cohen, 1998; Schaefer et al., 2009; Guterstam et al., 2011) and of body deformation illusions (Lackner, 1988; Ehrsson et al., 2005; Schaefer et al., 2007; Newport and Preston, 2010; Normand et al., 2011; Kilteni et al., 2012; Preston and Newport, 2012), as well as of full body ownership and out-of-body illusions (Lenggenhager et al., 2007, 2009; Petkova and Ehrsson, 2008; Aspell et al., 2009; lonta et al., 2011). In some cases, it has been noticed that incongruent multisensory information can be assimilated without destroying the illusion. For example, Slater et al. (2010) found that asynchronous visuotactile stimulation did not prevent a full body ownership illusion in an IVR setting where participants had 1PP over the virtual body and received visual sensorimotor congruent stimulation from head-tracking.

The first question that arises when comparing our results with those from previous studies concerns the reason why previous studies have repeatedly found that synchronous visuotactile (or headbased sensorimotor) stimulation was a necessary condition for eliciting a body ownership illusion, while we have found that this is not the case. There are two main points that may answer this question. First, the hypothesis that "the sole effect of congruent visuoproprioceptive cues, provided by a high degree of spatial overlap between the physical and virtual bodies, is a sufficient condition for inducing a full body ownership" has never been tested explicitly. The RHI paradigm intrinsically involved the use of visuotactile stimulation, with the control condition being the asynchronous mode rather than a "no touch" condition. This paradigm has been extended automatically to the case of full body illusions. In spite of this, it has been shown that the RHI can persist during period in which visuotactile stimulation was not delivered (Hohwy and Paton, 2010). The experimental design included nevertheless an initial phase of synchronous visuotactile stimulation that may have induced the illusion, which then persisted. Importantly, this experiment used stereo goggles that allowed a high degree of spatial matching between the real and virtual hands. In the same study, it was shown that after a period of synchronous visuotactile stimulation participants kept perceiving touch sensations when real touch was no longer delivered on the real hand, but appeared to be applied on the virtual hand. These findings are in perfect agreement with our results. Second, the reason for which other studies did not come to a similar conclusion can also be due to the fact that in most RHI experiments, apart from those using immersive stereo displays, the spatial locations of the real and artificial hand cannot perfectly coincide and consequently the visuoproprioceptive information provided is not fully congruent. It is interesting to note that all those experiments in which the ownership illusion was not disrupted by incongruent visuotactile stimuation (Hohwy and Paton, 2010; Slater et al., 2010; our experiment 1), used stereo vision, thus achieving a high degree of spatial overlap between the real and the virtual body (or body part) and, additionally, found that the asynchronous visuotactile information was not consciously perceived as wrong.

In order to have a unitary scenario that would allow the unification of the findings of different studies, we further need to address the following question: why other studies in which the physical and virtual bodies were highly spatially coincident (Petkova and Ehrsson, 2008; Petkova et al., 2011) found synchronous visuotactile stimulation to be necessary for the full body ownership illusion? To answer this question we move to the next section, where we discuss the role of the visual 
appearance of the virtual body.

\subsection{The Role of Body Appearance}

We have explicitly explored the role played by bodily appearance in experiment 3. Previous studies have already shown that non-humanoid shaped objects fail to be integrated and assimilated as pertaining to one's own body. The RHI does not work when a wooden no-hand-shaped object is stroked synchronously with the real hand (Tsakiris and Haggard, 2005). Tsakiris et al. (2010) further extended this finding, pointing out the need for an object to preserve precise, informative corporeal structural features in order to be integrable as one's own body part. Analogously, full body ownership illusions, as well as experimentally induced OBEs do not work when substituting the virtual body with a wooden block having the same dimensions of the fake body (Lenggenhager et al., 2007; Petkova and Ehrsson, 2008). Our experiment went beyond this by testing whether the level of realism of a humanoid body could modulate the intensity of the ownership illusion, and whether it interacts with other factors, such as the provided visuotactile stimulation.

The results from our experiment showed that participants experienced on average significantly higher levels of illusion when seeing a virtual body with common human features in term of skin texture and clothes, compared to the condition in which they were seeing a plastic mannequin. Nevertheless, the view of human-shaped mannequin does not completely dampen the illusion, consistent with previous reports (Petkova and Ehrsson, 2008; Petkova et al., 2011). When compared with findings from previous studies, our results further suggest that there may be an interaction of the body appearance with the visuotactile component. When seeing a mannequin body, synchronous visuotactile stimulation is found to be necessary to induce a vivid full body ownership illusion (Petkova and Ehrsson, 2008; Petkova et al., 2011). At the same time we found that, when the realism of the body is enhanced, a vivid illusion can occur without the need for additional synchronous visuotactile stimuation. Moreover, the illusion was preserved also when incongruent visuotacticle information was processed. This result supports the possibility of a top-down cognitive mechanism that modulates the way in which multisensory information is processed from the bottom-up perceptual stream. Our findings are in fact analogous to what has been established for the RHI: first the hand-object needs to pass a fitness test in terms of anatomical, volumetric and postural constraints (see Tsakiris et al., 2010 and reference therein). Once the fitness test is passed, other features enhancing the realism of the object to incorporate, e.g., the skin texture, can modulate the intensity of the illusion (Haans et al., 2008).

\subsection{A BASIC MODEL FOR THE FULL BODY OWNERSHIP ILLUSION}

In this section we sketch a speculative basic model for the neural underpinning of the full body ownership illusion on the basis of key findings from neurophysiological studies in the monkey. We first review the main relevant neurophysiological results that provide a rational for the proposed model. Graziano et al. (2000) reported finding visuoproprioceptive bimodal neurons in the area 5 
of the primate parietal cortex, whose properties are extremely relevant for the present discussion. These bimodal neurons respond to both the seen and the perceived (by proprioception) positions of a limb, even when the seen limb is fake. The first important property of such neurons is that they are sensitive to the visual content of the stimuli, so that their response is modulated by the position of an object in their visual RF only if the object contains the proper anatomical features of the limb that the same neurons encode by proprioception. Interestingly, the fake arm used in that study is extremely realistic, having been prepared by a taxidermist from a monkey of the same species. Visual and proprioceptive signals can be additive or may be combined in more complex fashion, according to the specific neuron. However, the overall averaged activity of this bimodal neuronal population shows a modulation associated with the relative positions of the fake and real arms, the activity being the highest when the locations of the two limbs are the closest. The third important property of such neurons is that their response is modulated by the relative position of the fake and real arms only when the fake arm location has a plausible position with respect to the rest of the body, meaning that no modulation was observed when the realistic fake arm was in a non-matching handedness position or in a backward orientation (with the hand being near the shoulder and the cut end extended outward). A further fundamental finding of the same study is the identification of a second population of trimodal neurons (responding to visual, tactile, and proprioceptive signals) in the same area 5 . Neurons in this population encode the position of the real arm by proprioception, but not that of a just-seen fake arm; they become sensitive to the view of the fake arm only when the latter is stroked synchronously (but not asynchronously) with the real arm. Area 5 have been also found to host neurons with large RF, which can be bilateral and can involve multiple body parts (Iwamura et al., 1994; Iwamura, 1998), making them plausible candidates for encoding information about the whole body. Other higher-ordered somatosensory areas have been shown to host bimodal and multimodal neuronal populations with large and bilateral RF. These include the ventral intraparietal (VIP) area and a polysensory zone in the precentral gyrus, hosting bimodal visuotactile neurons, as well as trimodal neurons that additionally responds to auditory or vestibular stimuli (Duhamel et al., 1998; Bremmer et al., 2002; Graziano and Cooke, 2006). A more comprehensive review of the neurophysiological studies of body representation in the monkey brain, which are relevant for the study of human body perception is beyond the scope of this paper and can be found in Blanke (2012).

In the following we propose a speculative basic model for the full body ownership illusion, influenced by the numerous analogies between the findings from neurophysiological recording in the monkey discussed above and the results from the work done on experimentally induced bodily illusions, including the main results of the present study. The same model would apply for the RHI. We propose that distinct bimodal and multimodal neuronal populations are responsible for the ownership illusion. A driving population of bimodal visuoproprioceptive neurons, with properties analogous to those of area 5 neurons in the monkey, would yield the minimal contribution necessary for the illusion to occur. Other bimodal and multimodal populations would have instead a secondary modulating effect. The visuoproprioceptive population activates when the seen body looks similar to and is located close to the real hidden body (i.e., when highly congruent static visuoproprioceptive correlations are provided).As for area 5 neurons in the monkey, this population activates only when the virtual body satisfies the main basic anatomical constraints in terms of shape and visual perspective. The higher the spatial coincidence between the virtual and the real 
body, the more intense would be the driven activity in this population and the resulting sense of ownership.Visuotactile, visual sensorimotor and other multimodal stimulations would trigger other bimodal and/or multimodal populations that have the effect of enhancing or dampening the sense of ownership, depending on whether they are delivered with proper correlations or not.

In this scenario we can isolate various steps for the "building-up" of an ownership illusion, encompassing most of the results that have been found in experimental studies on bodily illusions. If anatomical constraints are not satisfied in terms of body shape and visual perspective, no illusion occurs, because the driving visuoproprioceptive population is shut down, no matter whether other proper multimodal contingencies are provided. When anatomically constraints are satisfied, a high degree of spatial overlap between the virtual body and the real body could enhance the strength of the illusion. The additional contribution of the top-down effect from a high visual realism of the fake body could bring the illusion to saturation. In this condition, the reinforcing modulation of congruent multimodal correlations cannot be appreciated easily, because the illusion is already strong; moreover, the illusion can be sustained despite the dampening effect of incongruent multimodal stimulation (as in experiment 1), although severe and sustained incongruency could break the illusion completely. If anatomically constraints are met but the realism and colocation are not at a high degree, the driving visuoproprioceptive population will be activated, but with a moderate/low intensity that does not necessarily correspond to a perceptual illusion. In this condition, the modulating effects of additional multimodal stimulations can be critical for eliciting a vivid ownership illusion, when congruent, and more effective in breaking the illusion (if present), when incongruent [as in experiment 3, Petkova and Ehrsson (2008), and most of the RHI experiments].

In a recent review, Blanke (2012) has proposed that the changes in self-identification occurring during an OBE could correspond to changes in the size and position of the visual RFs of visuotactile trunkcentered neurons in VIP. Under the effect of synchronous back-stroking, these RFs would extend far enough to encode the fake body that comes to be part of the participant's peripersonal space (Maravita and Iriki, 2004). An additional subpopulation of trimodal neurons integrating visuotactle and vestibular signals would instead be responsible for changes in self-location and visual perspective. Taken together, the basic model we propose here for the full body ownership illusion and the model proposed for OBEs in Blanke (2012), would provide an evidence for the two types of full body illusions to be different perceptual illusions with different underlying patterns of neural activation.

\section{Conclusions}

The aim of our study was to identify the main perceptual cues underlying the full body ownership illusion and to determine their role and mutual interaction in the elicitation of the illusion. We have selectively manipulated visuotactile and visual sensorimotor stimulation, visual perspective, and the appearance of the virtual body.

We have found that having a first person perspective is an essential condition for experiencing the sense of ownership over the virtual body. When 1PP is provided over a realistic virtual body with a 
high degree of spatial overlap with the real body, the sole effect of congruent visuoproprioceptive cues can provide a sufficient condition for the illusion. In this condition, the additional contribution of congruent visuotactile and/or sensorimotor stimulation is indeed not necessary. Nonetheless, when the degree of spatial overlap (between the real and virtual body) and/or of the visual realism (of the fake body) is not high, congruent multisensory and/or sensorimotor cues are needed to trigger the illusion. In this case, such multimodal cues can be effective in both boosting the illusion, when congruent, and in suppressing it, when incongruent. We furthermore found that, when a high level of illusion is achieved by the synergic merging of various components, incongruent cues can be processed without breaking the illusion and can be perceived as not incorrect.

We discussed these findings showing that they are consistent with most of the previous results on part- and full-body ownership illusions. Motivated by a number of findings from neurophysiological recordings in the macaque monkey, we further propose a speculative basic model for the full body ownership illusion that accounts for most of the published results.

The present work advances our understanding of the critical mechanisms involved in the full body ownership illusion and provides useful implications for research in body-perception, selfconsciousness, and numerous virtual reality applications. 


\section{References}

Armel K. C., Ramachandran V. S. (2003). Projecting news. Presence Teleoper.Virtual Environ. 19, 291-301 sensations to external objects: evidence from skin conductance response. Proc. R. Soc. Lond. B Biol. Sci. 270,1499-1506

Duhamel J. R., Colby C. L., Goldberg M. E. (1998). Ventral intraparietal area of the macaque: congruent visual and Aspell J. E., Lenggenhager B., Blanke O. (2009). Keeping somatic response properties. J. Neurophysiol. 79, 126-136 in touch with one's self: multisensory mechanisms of selfconsciousness. PLoS ONE 4:e6488

Ehrsson H. H. (2007). The experimental induction of out-ofbody experiences. Science 317,1048

Berlucchi G., Aglioti S. M. (1997). The body in the brain: neural bases of corporeal awareness. Trends Neurosci. 20, Ehrsson H. H. (2012). The concept of body ownership and its $560-564$ relation to multisensory integration, in The New Handbook of Multisensory Processes, ed Stein B. E., editor. (Cambridge,

Berlucchi G., Aglioti S. M. (2010). The body in the brain MA: MIT Press), 775-792 revisited. Exp. Brain Res. 200, 25-35

Ehrsson H. H., Kito T., Sadato N., Passingham R. E., Naito Blanke O. (2012). Multisensory brain mechanisms of bodily E. (2005). Neural substrate of body size: illusory feeling of self-consciousness. Nat. Rev. 13, 556-571 shrinking of the waist. PLoS Biol. 3:e412

Blanke O., Metzinger T. (2009). Full-body illusions and Ehrsson H. H., Spence C., Passingham R. E. (2004). That's minimal phenomenal selfhood. Trends Cogn. Sci. 13, 7-13 my hand! Activity in premotor cortex reflects feeling of ownership of a limb. Science 305, 875-877

Blanke O., Mohr C. (2005) Out-of-body experience, heautoscopy, and autoscopic hallucination of neurological Fotopoulou A., Jenkinson P. M., Tsakiris M., Haggard P., origin implications for neurocognitive mechanisms of corporeal Rudde A., Kopelman A. D. (2011). Mirror-view reverses awareness and self-consciousness. Brain Res. Rev. 50,184- somatoparaphrenia: dissociation between first- and third199 person perspectives on body ownership. Neurophyscologia 49, 3946-3955

Bradley M. M., Codispoti M., Cuthbert B. N., Lang P. J. (2001). Emotion and motivation I: defensive and appetitive Graziano M. S., Cooke D. F., Taylor C. S. (2000). Coding the reactions in picture processing. Emotion 1, 276-298

location of the arm by sight. Science $290,1782-1786$

Botvinick M., Cohen J. (1998). Rubber hands 'feel' touch that Graziano M. S., Cooke D. F. (2006). Parieto-frontal eyes see. Nature 391, 756

interactions, peripersonal space, and the defensive behavior. Neuropsychologia 44, 845-859

Bremmer F., Klam F., Duhamel J. R., Ben Hamed S., Graf W. (2002). Visual-vestibular interactive responses in the Graziano M. S. A., Botvinik M. M. (2002). How the brain macaque ventral intraparietal area (VIP). Eur. J. Neurosci.16, represents the body: insights from neurophysiology and 1569-1586 psychology, in Common Mechanisms in Perception and Action, Attention and Performance XIX, eds Prinz W., Cacioppo J., Tassinary L., Berntson G. (2007). Handbook Hommel B., editors. (Oxford: Oxford University Press; ), of Psychophysiology. Cambridge: Cambridge University 136-157

Press

Giummarra M. J., Gibson S. J., Georgiou-Karistianis N., Cohen L., Manion L., Morrison K. (2011). Research Methods Bradshaw J. L. (2007). Central mechanisms in phantom limb in Education, 6th Edn London: Routledge-Falmer, 386-388 perception: the past, present and future. Brain Res. Rev. 54, ISBN13: 978-0-415-58335-0 219-232

de la Peña N., Weil P., Llobera J., Giannopoulos E., Guterstam A., Ehrsson H. H. (2012). Disowning one's seen Pomes A., Spanlang B., et al. (2010). Immersive journalism: real body during an out-of-body illusion. Conscious. Cogn. immersive virtual reality for the first-person experience of 21,1037-1042 
Guterstam A., Petkova V. I., Ehrsson H. H. (2011). The illusion Makin T. R., Holmes N. P., Ehrsson H. H. (2008). On the other of owning a third arm. PLoS ONE 6:e17208 10.1371/journal. hand: dummy hands and peripersonal space. Behav. Brain Res. pone.0017208

$191,1-10$

Haans A., ljsselsteijn W. A., de Kort Y. A. (2008). The effect Maravita A., Iriki A. (2004). Tools for the body (schema). of similarities in skin texture and hand shape on perceived Trends Cogn. Neurosci. 8, 79-86

ownership of a fake limb. Body Image 5, 389-394

Newport R., Preston C. (2010). Pulling the finger off disrupts Hohwy J., Paton B. (2010). Explaining away the body: agency, embodiment and peripersonal space. Perception 39 , experiences of supernaturally caused touch and touch on 1296-1298

non-hand objects within the rubber hand illusion. PLoS ONE 5:e9416

Normand J. M., Giannopoulos E., Spanlang B., Slater M. (2011). Multisensory stimulation can induce an illusion of larger

lonta S., Heydrich L., Lenggenhager B., Mouthon M., Fornari belly size in immersive virtual reality. PLoS ONE 6:e16128

E., Chapuis D., et al. (2011). Multisensory mechanisms in

temporo-parietal cortex support self-location and first-person Petkova V. I., Ehrsson H. H. (2008). If I were you: perceptual perspective. Neuron 70, 363-374

illusion of body swapping. PLoS ONE 3:e3832

IwamuraY. (1998). Hierarchical somatosensory processing. Petkova V. I., Khoshnevis M., Ehrsson H. H. (2011). The Curr. Opin. Neurobiol. 8, 522-528

perspective matters! Multisensory integration in ego-centric reference frames determines full-body ownership. Front.

Iwamura Y., Iriki A., Tanaka M. (1994). Bilateral hand Psychol. 2:35

representation in the postcentral somatosensory cortex.

Nature 369, 554-559

Preston C., Newport R. (2012). How long is your arm? Using multisensory illusions to modify body image from the third

Kalckert A., Ehrsson H. H. (2012). Moving a rubber hand that person perspective. Perception 41, 247-249

feels like your own: a dissociation of ownership and agency.

Front. Hum. Neurosci. 6:40

Pomes A., Giannopoulos H., Slater M. (2012). Touching the void: exploring virtual objects through a vibrotactile glove, in

Kilteni K., Normand J. M., Sanchez-Vives M. V., Slater M. IEEEVR - OTSVR 2012, California (International Journal of (2012). Extending body space in immersive virtual reality: a very Virtual Reality, in press).

long arm illusion. PLoS ONE 7:e40867

Schaefer M., Flor H., Heinze H. J., Rotte M. (2007). Lackner J. R. (1988). Some proprioceptive influences on the Morphing the body: illusory feeling of an elongated arm affects perceptual representation of body shape and orientation. Brain somatosensory homunculus. Neuroimage 36, 700-705 $111,281-297$

Schaefer M., Heinze H., Rotte M. (2009). My third arm: shifts Lenggenhager B., Halje P., Blanke O. (2011). Alpha band in topography of the somatosensory homunculus predict feeling oscillations correlate with illusory self-location induced by virtual of an artificial supernumerary arm. Hum. Brain Mapp. 30, reality. Eur. J. Neurosci. 33, 1935-1943

1413-1420

Lenggenhager B., Mouthon M., Blanke O. (2009). Spatial Spanlang B., Normand J. M., Giannopoulos H., Slater M. aspects of bodily self-consciousness. Conscious. Cogn. 18, (2010). GPU based detection and mapping of collisions for haptic 110-117 rendering in immersive virtual reality, in IEEE International Symposium on Haptic Audio Visual Environments and

Lenggenhager B., Tadi T., Metzinger T., Blanke O. (2007). Games (HAVE), 2010 (Phoenix,AZ), 41-44

Video ergo sum: manipulating bodily self-consciousness. Science 317, 1096-1099

Slater M., Spanlang B., Sanchez-Vives M., Blanke O. (2010). First person experience of body transfer in virtual reality. PLoS

Lloyd D. M. (2007). Spatial limits on referred touch to an alien limb may reflect boundaries of visuo-tactile peripersonal space surrounding the hand. Brain Cogn. 64, 104-109

Tajadura-Jim'enez A., Grehl S., Tsakiris M. (2012). The other 
in me: interpersonal multisensory stimulation changes the mental representation of the self. PLoS ONE 7:e40682

Tsakiris M., Prabhu G., Haggard P. (2006). Having a body versus moving your body: how agency structures body-ownership. Conscious. Cogn. 15, 423-4321

Tsakiris M. (2010). My body in the brain: a neurocognitive model of body-ownership. Neuropsychologia 48, 703-712

Tsakiris M., Carpenter L., James D., Fotopoulou A. (2010). Hands only illusion: multisensory integration elicits sense of ownership for body parts but not for non-corporeal objects. Exp. Brain Res. 204, 343-352

Tsakiris M., Haggard P. (2005). The rubber hand illusion revisited: visuotactile integration and self-attribution. J. Exp. Psychol. Hum. Percept. Perform. 31, 80-91

Vallar G., Ronchi R. (2009). Somatoparaphrenia: a body delusion.A review of the neuropsychological literature. Exp. Brain Res. 192, 533-551 



\section{CHAPTER 3}

\section{The Sense of Body OWnership Relaxes Temporal Constraints for Multisensory Integration}

Related publication:

The sense of body ownership relaxes temporal constraints for multisensory integration.

Maselli,A., Kilteni, K., López-Moliner, J., and Slater, M.

Sci. Rep. 6, 30628, 2016 


\section{Abstract}

Experimental work on body ownership illusions showed how simple multisensory manipulation can generate the illusory experience of an artificial limb as being part of the own-body. This work highlighted how own-body perception relies on a plastic brain representation emerging from multisensory integration. The flexibility of this representation is reflected in the short-term modulations of physiological states and perceptual processing observed during these illusions. Here, we explore the impact of ownership illusions on the temporal dimension of multisensory integration. We show that, during the illusion, the temporal window for integrating touch on the physical body with touch seen on a virtual body representation, increases with respect to integration with visual events seen close but separated from the virtual body. We show that this effect is mediated by the ownership illusion. Crucially, the temporal window for visuotactile integration was positively correlated with participants' scores rating the illusory experience of owning the virtual body and touching the object seen in contact with it. Our results corroborate the recently proposed causal inference mechanism for illusory body ownership. As a novelty, they show that the ensuing illusory causal binding between stimuli from the real and fake body relaxes constraints for the integration of bodily signals. 


\section{INTRODUCTION}

During body ownership illusions (BOls) healthy adults experience artificial limbs or bodies as part of their own body representation. BOls are thought to rely on the integration of synchronous but independent perceptual stimuli, for example visual stimuli seen on the dummy hand and tactile ones felt on the real hand in the case of the Rubber Hand Illusion (RHI) (Botvinik and Cohen, 1998). Similar multisensory manipulations have been shown effective to induce the illusion of ownership over supernumerary limbs (Folegatti et al., 2012; Newport et al., 2010; Guterstam et al., 2011) and artificial bodies (Petkova and Ehrsson, 2008; Maselli and Slater, 2013), demonstrating how our ownbody perception relies on a plastic brain representation emerging from multisensory integration (Graziano and Botvinik, 2002; Takiris et al., 2010; Makin et al., 2008; Kilteni et al., 2015).

Evidence for the flexibility of this brain representation comes not only from vivid subjective reports about illusory ownership experienced over artificial limbs and full bodies, whose appearance can importantly deviate from the one of the real counterpart, e.g. in size (Kilteni et al., 2012; Pavani et al., 2007; van der Hoort et al. 2011), skin color (Farmer et al, 2012; Peck et al., 2013), age (Banakou et al., 2013) and realism (Bertamini and O'Sullivan 2014; Martini et al., 2015). This flexibility is also robustly supported by a number of short-term modulations of behavior and physiological states. As representative examples, it has been reported that during a RHI the temperature of the "substituted" hand drops (Moseley et al., 2011) together with its tactile sensitivity (Moseley et al., 2011; Zopf et al., 2011; Zeller et al., 2014), and that histamine reactivity to noxious stimuli increases (Barnsley et al., 2011). On the behavioral side, it was shown, for example, how experiencing ownership over a darkskin body can reduce implicit racial-bias (Peck et al. 2013; Maister et al., 2013), or how ownership over a child body can affect size perception and induce implicit attitude changes (Banakou et al., 2013). These are just some examples of the profound impact on self-perception and behavior that can occur during body ownership illusions, over time scales of few tens of seconds.

Multisensory integration has been at the core of research into body ownership illusions since their first reports (Botvinik and Cohen, 1998; Graziano and Botvinik, 2002), and is indeed regarded as the basic causal mechanism for their emergence (Makin et al., 2008; Tsakiris 2010; Ehrsson 2011). However, the converse has not been previously studied, which is concerned with how BOls modulate the processing and merging of cross-modal stimuli. On the other hand, extensive research in multisensory perception shed light over the intrinsic flexibility of multisensory integration processing, which allows adapting efficiently to the continuously changing environment. In this study we face this gap and explore potential effects of ownership illusions on multisensory processing.

Integrating the different multimodal signals streaming from an object or an event is essential for having a coherent and meaningful perceptual experience, but this is not a straightforward task for the central nervous system (CNS). In fact, due to differences in transduction and transmission characteristic times, the temporal lag for a signal to trigger a response in the corresponding primary sensory cortex can significantly vary across modalities. For example, due to the long transduction time of photoreceptors (Kuffler et al., 1953), which is longer with respect to that of tactile receptors, 
a truly synchronous visuotactile event typically triggers activity in SI in advance with respect to activity triggered in V1. Furthermore, the relative delay can change importantly according to the lighting conditions and the ambient temperatures that affects transduction (Barnett-Cowan and Harris, 2009), and also depending on the body part that is touched, which affects transmission time of tactile stimuli (Harrar and Harris, 2005). Therefore, in order to assure a coherent perception of the environment, the CNS has to flexibly compensate for such differences by modulating the temporal window on which multisensory integration is effective (Kopinska and Harris, 2004; Harris et al., 2010; Keetels andVroomen, 2012). One of the factors that efficiently modulates the temporal window for multisensory integration is "causal binding", also referred to as "unity assumption". Having implicit knowledge of the existence of a common origin for two sensory signals was indeed shown to facilitate integration. In the case of audiovisual speech perception, larger temporal lags are indeed tolerated (perceived as synchronous) under the assumption of a common cause for the visual and auditory signals (Vatakis and Spence, 2007).

In the light of the results discussed, we argue that body ownership illusions could act as a "causal binding" factor for stimuli seen on the fake body and independent somatosensory signals, and therefore that BOls could modulate multisensory processing.As a further support to this proposal, a number of studies showed that, during BOls, visual threats to the artificial body trigger enhanced neurophysiological correlates of anxiety (Armel and Ramachandran, 2002; Ehrsson et al., 2004) thus revealing how visual stimuli on the fake body are processed as if seen on the own physical body. In the present work, we focus on the temporal constraints for visuotactile integration. We specifically hypothesized that, during the illusion, there is an expansion of the temporal window within which visual (on the fake body) and tactile (on the physical body) stimuli are perceived as simultaneous.

To test this hypothesis we performed two experiments. In Experiment 1, we assessed whether the assumption of a common origin - for a tactile stimulus and a correspondent visual cue on the fake body - could expand the temporal window of integration in the visuotactile domain, similarly to what has been found for the audiovisual domain (Vatakis and Spence, 2007; Vatakis et al., 2008). In Experiment 2, we tested whether this "causal binding" effect emerges as a consequence of the body ownership illusion itself.

We relied on a temporal order judgment (TOJ) task as an established procedure to estimate the temporal window of integration. In TOJ tasks, pairs of target stimuli with varying temporal separations (typically referred to as Stimulus Onset Asynchrony - SOA) are presented to participants that have to judge which stimulus came first. The analysis of how responses vary across the tested range of SOAs, allows estimating two main quantities of interest: the Point of Subjective Simultaneity (PSS) - a measure of the average time one stimulus has to precede the other in order for the two to be perceived as simultaneous (Shore et al., 2001; Kennett et al., 2001) - and the Just Noticeable Difference (JND) - a measure of the temporal window in which the two stimuli are perceived as simultaneous (Yamamoto and Kitazawa, 2001; Shore et al., 2005; Gallace and Spence, 2005). In particular, for a multimodal TOJ task, the JND can be regarded as a proxy for the temporal window of multisensory integration (Vatakis and Spence, 2007; Mégevand et al., 2013; Vroomen and Keetels, 2010), and as such it is the quantity of interest for the current 
study.

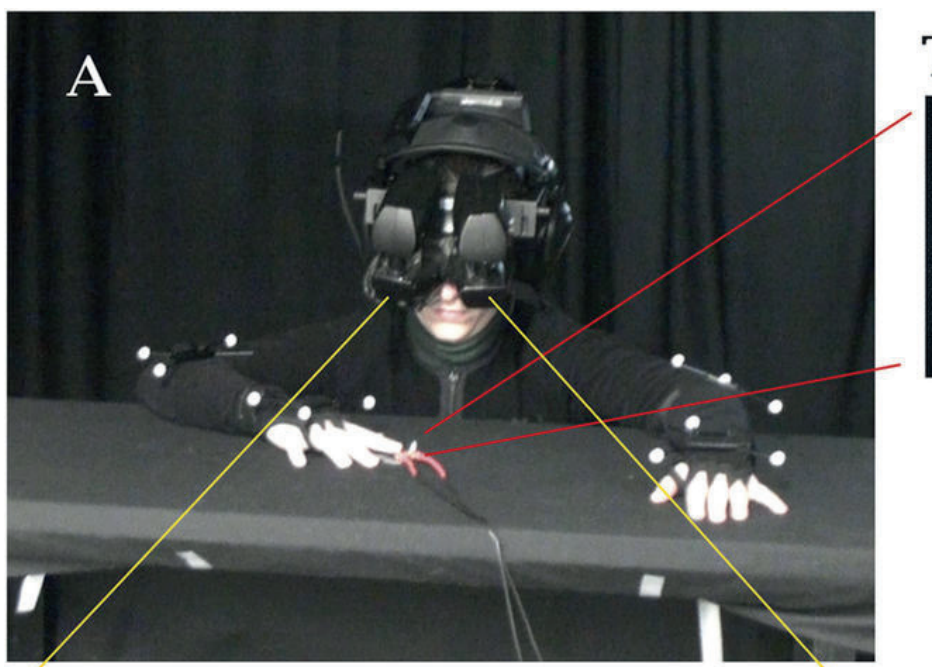

\section{TACTILE CUE}

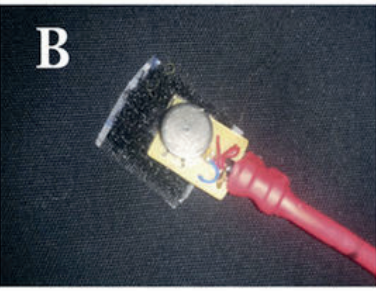

50 ms vibration

\section{VISUAL CUE $50 \mathrm{~ms}$ wheel rotation}
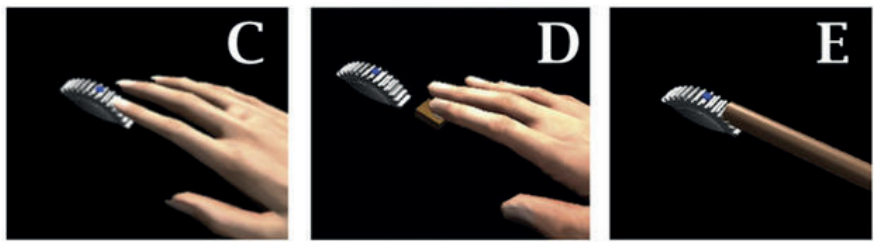

Figure 1: Experimental setup. (A) Participants performed a visuotactile Temporal Order Judgment (TOJ) task, while wearing head-mounted display. (B) Piezoelectric motor used to deliver vibrotactile stimuli.The visual stimulus was a $50 \mathrm{~ms}$ rotation of a virtual geared-wheel: the geared-wheel was seen (C) in contact with the virtual finger (Exp. 1:Touch condition; Exp. 2: Body condition), (D) separated from the virtual finger (Exp. 1: No-Touch condition) or (E) touching a wooden stick (Exp. 2: Stick condition). The 3D graphics elements were designed with Autodesk ${ }^{\circledR} 3 \mathrm{ds}$ Max ${ }^{\circledR}$ and controlled through the Unity ${ }^{\circledR}$ software platform.

We implemented a visuotactile TOJ task that participants had to perform while wearing a headtracked head-mounted display (HMD) streaming a digital 3D replica of the experimental room. When looking down towards their real occluded body, participants could see either a virtual body or two wooden sticks, depending on the experimental condition. Participants performed the TOJ task while resting their arms on a Table (Fig. 1A). The tactile stimulus was a $50 \mathrm{~ms}$ vibration delivered to the right index fingertip.The visual stimulus was a rotation $(50 \mathrm{~ms}$ duration) of a virtual geared-wheel 
(Supplementary Video S1 ${ }^{1}$ ). For further details on the TOJ task implementation and on methods for estimating the JNDs, we refer the reader to Materials and Methods.

In Experiment 1, we explicitly manipulated the causal relationship between the visual and tactile stimuli. Participants had a first person perspective over a gender-matched virtual body that was spatially coincident with their real body. This configuration is known to be sufficient for inducing an ownership illusion through congruent visuo-proprioceptive cues (Maselli and Slater, 2013, 2014). Participants performed the TOJ task in two conditions: the geared-wheel was seen either in contact with the index finger of the virtual body (Touch) (Fig. 1C) or separated from it by $6 \mathrm{~mm}$ (No-Touch) (Fig. 1D, Supplementary Video S1 $^{1}$ ).

In Experiment 2, we tested whether this illusory "causal binding" is mediated by the ownership illusion. Participants performed the TOJ task in two conditions with different manipulations of illusory body ownership. One condition (Body) was the same as the Touch one in Experiment 1 (Fig. 1C). In the other (Sticks), the sense of ownership was inhibited by displaying two wooden sticks placed on the Table and spatially coincident with the arms of the participants during the TOJ task, with the tip of the right stick being in touch with the geared-wheel (Fig. 1E, Supplementary Video $\left.S 1^{1}\right)$. This manipulation of the shape of the virtual body was used since successful induction of a $\mathrm{BO}$ requires that the fake body or body part should have a humanoid shape (Petkova and Ehrsson, 2008; Tsakiris et al., 2010).

\begin{tabular}{|l|l|}
\hline Tag & Questionnaire Item \\
\hline Ownership & $\begin{array}{l}\text { During the temporal order judgment task I felt as if the } \\
\text { virtual hand (stick) I was looking at was my own hand }\end{array}$ \\
\hline Touch & $\begin{array}{l}\text { During the temporal order judgment task I felt as if my } \\
\text { right index finger was touching the virtual wheel }\end{array}$ \\
\hline Cause & $\begin{array}{l}\text { During the temporal order judgment task I felt as if } \\
\text { the rotation of the virtual wheel produced the tactile } \\
\text { stimuli on my finger }\end{array}$ \\
\hline Move & $\begin{array}{l}\text { I felt as if the virtual arms (sticks) were following the } \\
\text { movements of my own arms }\end{array}$ \\
\hline Ctrl & I felt as if I had two right hands \\
\hline
\end{tabular}

Table 1: Questionnaire items. The Table lists the six items presented to participants at the end of each experimental session. The items were presented in a randomized order across participants and experimental conditions. Participants had to indicate their level of agreement with each of the statement, on a Likert scale from -3 to 3.The text in parentheses was used to replace the italic text in the Stick condition in Experiment 2.

1 Supplementary videos can be found at: https://www.nature.com/articles/srep30628\#supplementary-information 
A six-item questionnaire, presented at the end of each experimental session, was administered to assess the subjective illusory experience (Table 1). Each item was scored on a -3 to +3 Likert scale, according to the level of agreement with the statement.

\section{Results}

In Experiment 1 we expected to find higher JND in the Touch condition, because seeing the virtual finger touching the moving geared-wheel during an ownership illusion should provide hints for a common origin of the two target stimuli. This was indeed the case for 10 out of 14 subjects, with an average JND difference across conditions of $46 \mathrm{~ms}$ (Fig. 2A, B). Data from all 14 participants were analyzed using a Generalized Linear Mixed Model (GLMM). The GLMM analysis revealed a significant difference across conditions in the slopes of the fitted psychometric curves $(p<0.0001)$. Corresponding bootstrap estimates of the JNDs were $155 \mathrm{~ms}(95 \% \mathrm{Cl}=142$ to $168 \mathrm{~ms})$ and $127 \mathrm{~ms}$ ( $95 \% \mathrm{Cl}=117$ to $136 \mathrm{~ms}$ ) in the Touch and No-Touch conditions respectively (Fig. 2C).

Results from the subjective scores to the six-item questionnaire are summarized in Table 2.Participants reported a strong illusion of virtual body ownership (median scores of 2 ) in both conditions ( $p>0.48)$. The feeling that the wheel was directly touching their finger was positively reported only in the Touch condition $(p=0.002$; median scores of 1.5 for Touch and -3 for No-Touch). Consistently, the feeling of the virtual wheel being the origin of the vibrotactile TOJ cues was higher in the Touch condition (median scores of 1 for Touch and -0.5 for No-Touch), although the difference between conditions did not reach the significance level $(p=0.06)$. These results support our hypothesis that the illusory experience of touching the virtual wheel acts as a "causal binding" factor, expanding the temporal window for visuotactile integration.

\section{Table 2: Questionnaire Results}

\begin{tabular}{|c|c|c|c|c|c|}
\hline \multicolumn{6}{|c|}{ Experiment 1} \\
\hline Item & Touch & No-Touch & Touch-No-Touch & p-value & PS $_{\text {dep }}$ \\
\hline Ownership & $2(1)$ & $2(1.5)$ & $0(0.75)$ & 0.48 & 0.53 \\
\hline Touch & $1.5(4.5)$ & $-3(1)$ & $2.5(3.75)$ & 0.002 & 0.96 \\
\hline Cause & $1(2)$ & $-0.5(4.5)$ & $1(3)$ & 0.06 & 0.75 \\
\hline \multirow[t]{2}{*}{ Move } & $3(1)$ & $3(1)$ & $0(0.75)$ & 0.78 & 0.46 \\
\hline & $-3(1.75)$ & $-3(0)$ & $0(0.75)$ & 0.10 & 0.64 \\
\hline \multicolumn{6}{|c|}{ Experiment 2} \\
\hline Item & Body & Stick & Body - Stick & p-value & PS $_{\text {dep }}$ \\
\hline Ownership & $2(1)$ & $-2(3)$ & $3(4)$ & 0.005 & 0.84 \\
\hline Touch & $1.5(3)$ & $-2(3.75)$ & $1.5(3)$ & 0.01 & 0.79 \\
\hline Cause & $1(1.75)$ & $1(3.5)$ & $0(1.75)$ & 0.12 & 0.61 \\
\hline Move & $3(1)$ & $-3(1.75)$ & $5(2)$ & 0.001 & 0.06 \\
\hline Ctrl & $-3(0)$ & $-3(0)$ & $0(0)$ & 0.58 & 0.50 \\
\hline
\end{tabular}



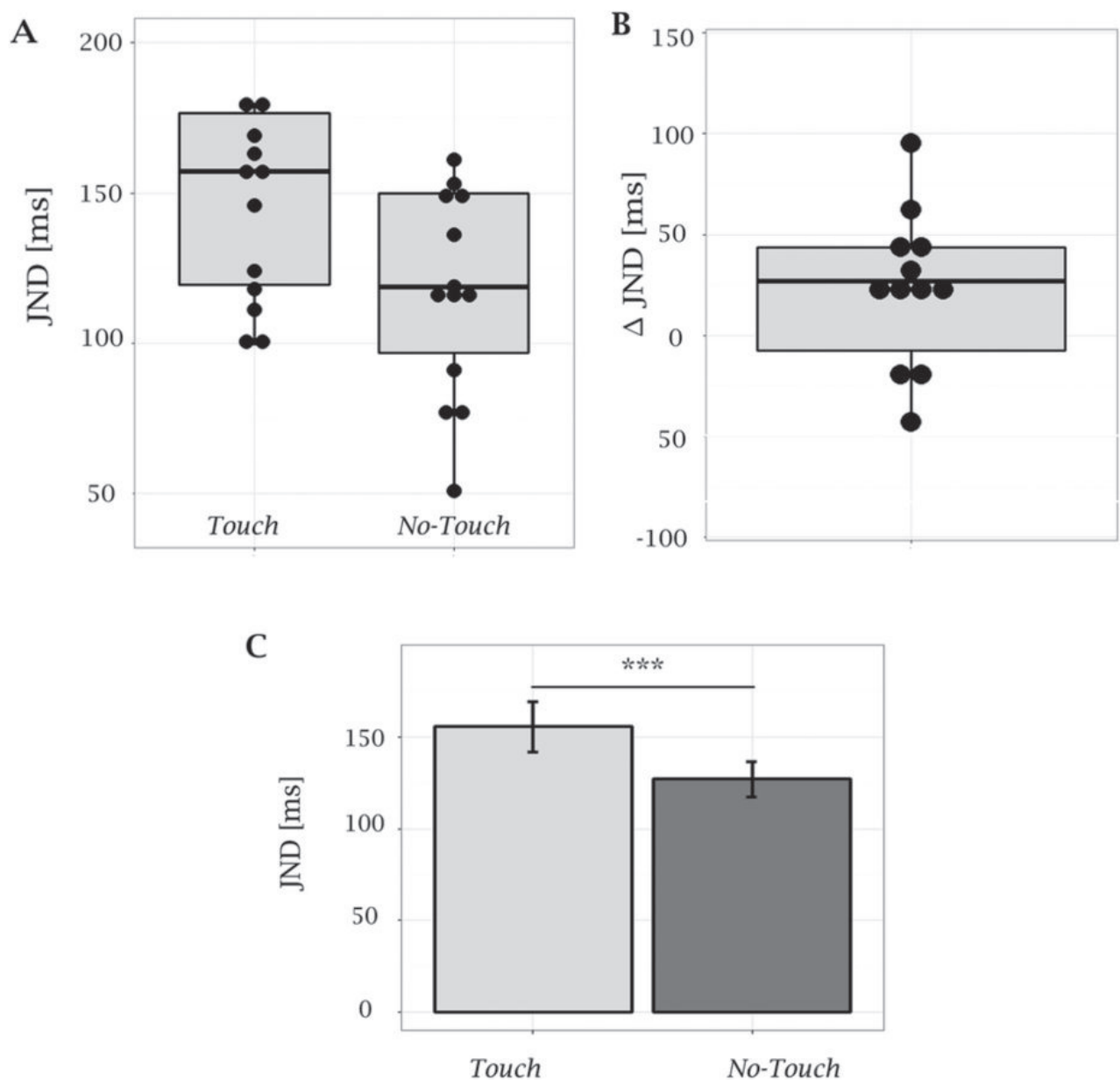

Figure 2: Results from Experiment 1. (A) Boxplot showing the distribution of JND estimates from individual fits in the two experimental conditions; JND estimates from single subjects are overplotted as scatter points. (B) Boxplot showing the distribution of JND differences in the two conditions $\left(\triangle J N D=J N D_{\text {Touch }}-J N D_{\text {No- }}\right.$ Touch), estimated from individual fits; $\triangle J N D$ for single subjects are overplotted as scatter points. For 10 out 14 participants the JND was higher in the Touch condition. The mean value of individual $\triangle J N D$ was $46.6 \mathrm{~ms}$. (C) JNDs estimates $(n=14)$ from Genelized Linear Mixed Model (Bootstrap method) were equal to $127 \mathrm{~ms}$ and $155 \mathrm{~ms}$ in the No-Touch and Touch conditions respectively. Vertical bars represent the $95 \% \mathrm{Cl}$ estimated with the bootstrap method67. GLMM analysis revealed a significant difference in across conditions $(p<0.0001)$. 

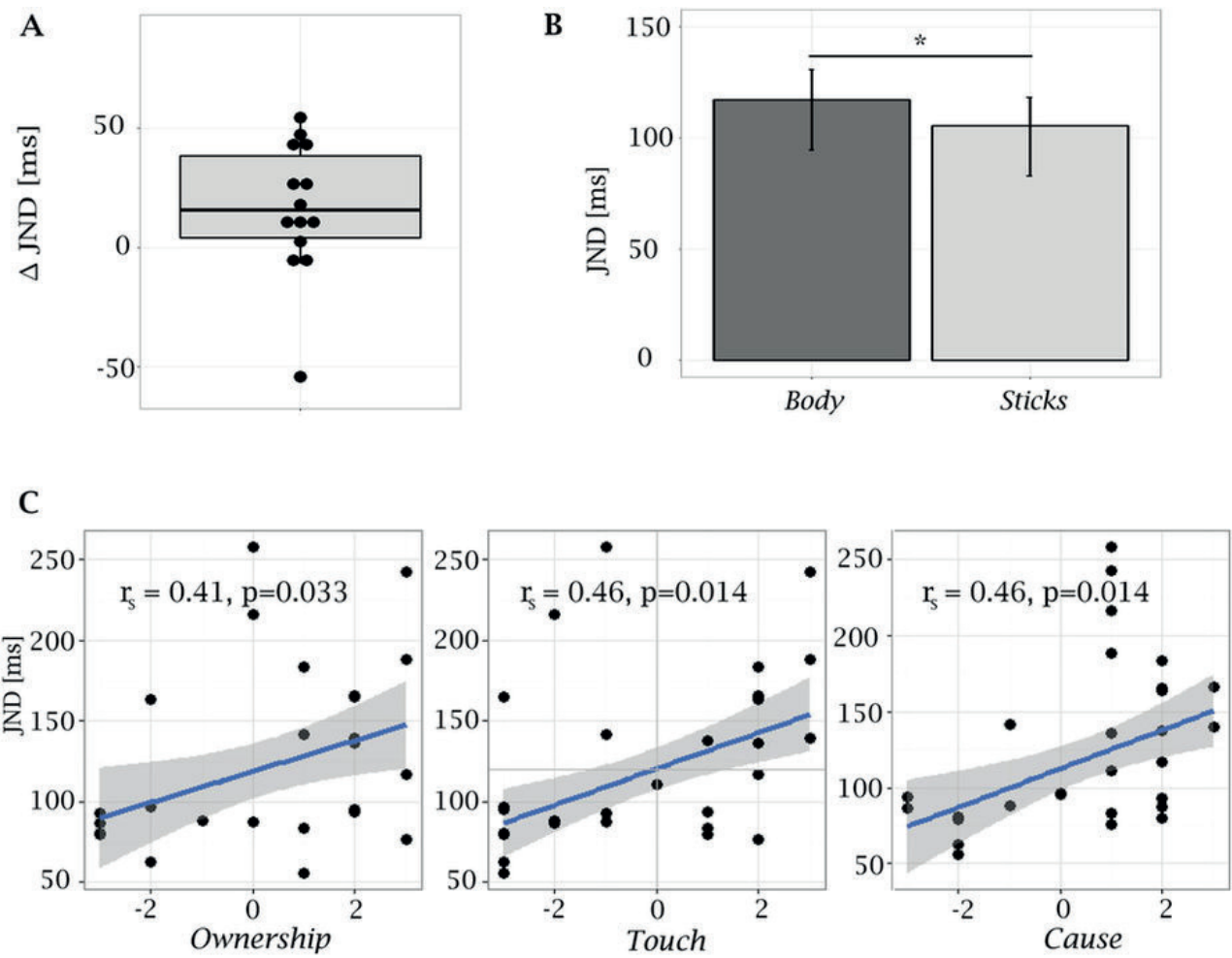

Figure 3: Results from Experiment 2. (A) Boxplot showing the distribution of JND difference in the two conditions $\left(\triangle J N D=J N D_{\text {Body }}-J N D_{\text {sticks }}\right)$, estimated from individual fits; $\triangle J N D$ for single subjects are overplotted as scatter points. For 11 out of 14 participants the JND was higher in the Body condition. The mean value of individual $\triangle J N D$ was $16.3 \mathrm{~ms}$. (B) JNDs estimates $(n=14)$ were equal to $127 \mathrm{~ms}$ and $155 \mathrm{~ms}$ in the Stick and Body conditions respectively. Vertical bars represent the $95 \% \mathrm{Cl}$ estimated with the bootstrap method67. GLMM analysis revealed a significant difference across conditions $(p=0.011)$. (C) JND estimates from individual fits are plotted as a function of subjective scores given to the "Ownership" (left panel), "Touch" (central panel), and "Cause" (right panel) questionnaire items (full statements listed in Table 1), together with the robust linear fits and associated $95 \% \mathrm{Cls}$. Spearman correlation analysis revealed significant positive correlations for the three cases.

In Experiment 2 we explicitly manipulated illusory body ownership to test the hypothesis that the illusion itself mediates the "causal binding" effect observed in Experiment 1. Results from the subjective scores to the questionnaire items are summarized in Table 2. Participants experienced a strong sense of ownership only in the Body condition (median scores of 2 for Body and -2 for Stick; $\mathrm{P}=0.005$ ). Analogously, the reported sensation of touching the geared-wheel (touch item) was high 
only for the Body condition (median scores of 1.5 for Body and -2 for Stick; $p=0.01$ ). Contrary to our expectation, this was not reflected in a significant difference for the reported sensation that the geared-wheel was the cause of the vibrotactile stimuli during the TOJ (cause item: $p=0.12$ ).

Importantly, for 11 of the 14 participants the JND was larger in the Body condition, with an average JND difference across conditions of $16 \mathrm{~ms}$ (Fig. 3A). Analysis of the 14 subjects performance using GLMM showed a significant difference across conditions for the fitted slope of the psychometric curves $(p=0.011)$, with bootstrap estimates of the JNDs being $117 \mathrm{~ms}(95 \% \mathrm{Cl}=95-131 \mathrm{~ms})$ and $105(95 \% \mathrm{Cl}=83-118 \mathrm{~ms})$ in the Body and Sticks conditions respectively (Fig. 3B). Crucially, the JND was positively correlated with subjective scores rating the sense of ownership $\left(r_{s}=0.41, p=0.033\right)$, the illusory sensation of touching the geared-wheel $\left(r_{s}=0.46, p=0.014\right)$, and of the geared-wheel being the cause of the vibrotactile target cues $\left(r_{s}=0.46, p=0.014\right)(F i g .3 C)$. This latter result may seem at odd with the unexpected lack of significant difference across conditions in the cause item scores. In fact, according to the rationale of our hypothesis, the illusion of ownership (triggered by the view of the spatially overlapping virtual body and reinforced by synchronous visuomotor stimuli) would generate the illusion of touching the virtual geared-wheel. This illusory touch would then trigger the illusory experience of the geared-wheel being the origin of the vibrotactile stimuli and in turns would relax temporal constraints for visuotactile integration (measured in terms of JNDs).

Results from Experiment 2 thus support our hypothesis, indicating that the effect of "causal binding" on the temporal window of visuotactile integration revealed in Experiment 1 is selectively mediated by the sense of ownership experienced toward the virtual body.

Taken together results from Experiment 1 and 2 highlight the reciprocal connection between multisensory integration and body ownership: multisensory integration builds the body ownership illusion, while the illusion, once induced, modulates subsequent multisensory processing.

\section{Discussion}

Spatiotemporal correlations of concurrent stimuli from different sensory channels and the motor system provide an essential contribution to self-perception and self-recognition (Gibson, 1986; van den Bos and Jeannerod, 2002; Rochat, 1998). Experimental protocols for ownership illusions rely on this principle: the spatiotemporal congruence of cross-modal bodily signals triggers the integration of truly independent stimuli, giving rise to the illusory experience that an external object is part of the own-body (Maselli and Slater, 2013; Makin et al., 2008; Ehrsson 2010; Botvinik 2004).

Here we provide novel experimental evidence that illusory ownership modulates multisensory integration. The results of the two experiments combined show that during the illusion the temporal window for visuotactile integration of body related cues expands. In the first experiment, we induced an ownership illusion over a virtual body so to be able to dissociate visual and tactile bodily signals: participants could see a rotating geared-wheel in touch or not with the virtual finger while 
receiving tactile stimuli on the real fingertip. When the finger was seen in touch with the moving object, the estimated temporal window for visuotactile integration was wider. This showed that when pairs of visuotactile bodily stimuli are attributed to the same cause, the temporal constraints for their integration get relaxed.

An alternative interpretation would be that visuotactile integration is inhibited in the No-Touch condition because of the spatial offset between visual and the tactile stimuli. While in the Touch condition the visual and tactile stimuli were aligned in space, in the No-Touch condition a small offset $(6 \mathrm{~mm})$ was introduced between the two, as the virtual finger was slightly displaced (with respect to the real one) so to be seen separated from the virtual wheel. Previous studies have shown that the perceived distance between target-stimuli can significantly affect TOJ performances, with better performances found for more distant stimuli (Shore et al., 2005; Shore and Spence,2005). Notwithstanding, in our case the change in distance between the visual and tactile stimuli across conditions was extremely small, and of the same order of magnitude of the intrinsic precision of the hand position sense (van Beers et al., 1998). Therefore the introduced offset should not significantly affect the perceived location of the tactile stimuli, remapped in external space towards the location of the slightly displaced virtual finger. It is worth noticing that previous studies reported changes in JND, as estimated from TOJ performances, of the order of $10 \mathrm{~ms}$ for an actual change in the targetstimuli distance of about $1 \mathrm{~m}$ (Shore et al., 2005), while here we found a change in JND almost three times larger for an actual change in distance of just $6 \mathrm{~mm}$. We can therefore conclude that the change in JND we found is not an effect of different displacements between target stimuli, but is instead due to the illusory causal binding elicited in the Touch condition and absent in the No-Touch condition.

In the second experiment, we manipulated illusory body ownership to show that the effect of causal binding observed in Experiment 1 is selectively modulated by the sense of ownership. We found that, if the sense of ownership is inhibited (by showing a wooden stick instead of the virtual hand touching the geared-wheel) the temporal window for visuotactile integration is indeed smaller with respect to the case in which the body ownership illusion was strong.

All together, results from our two experiments suggest that the relaxation of temporal constraints for multisensory integration observed in body ownership illusions is driven by a causal mechanism that binds together visual stimuli on the fake body and tactile stimuli on the physical body. Interestingly, our results could explain why participants undergoing an intense ownership illusion fail to notice asynchronies in visuotactile stimulation that are otherwise (in the absence of the illusion) detected (Maselli and Slater, 2013). Furthermore, the present results provide robust experimental support to previous proposals suggesting that the illusion can be sustained despite exposure to asynchronous visuotactile stimulation (Maselli and Slater, 2013; Kilteni et al., 2015).

Our results fit well within the framework provided by Bayesian causal inference models for illusory ownership (Kilteni et al., 2015; Samad et al., 2015). According to such models, the illusion arises when the brain associates a higher than chance probability to the existence of a single cause (the own-body) for all the incoming sensory input: the visual from the fake body and the somatosensory/ motor from the physical body. Importantly, the causal inference approach predicts that, under the assumption of a common cause, the integration of cross-modal sensory stimuli is facilitated 
(Körding et al., 2007; Shams and Beierholm, 2010; Parise et al., 2012). This prediction is indeed corroborated in the present study that showed facilitation in terms of an expanded temporal window for visuotactile integration.

The influence of causal binding on temporal aspects of multisensory perception has been previously reported. In the audiovisual domain, similar expansions of the temporal window of integration have been found, but only for functionally relevant stimuli such as speech (Vatakis and Spence, 2007 Vatakis et al., 2008). Similarly, intentional binding - a form of causal binding manipulated through voluntary motor actions - has been shown to anticipate the conscious perception of auditory stimuli (Haggard et al., 2012) and to suppress color-motion asynchronies (Corveleyn et al., 2012), otherwise observed in the perception of truly simultaneous changes in the color and position of an object (Moutoussis and Zeki, 1997). These results highlight the predictive nature of causal binding effects: predictions are indeed an intrinsic component of both voluntary motor control (Wolpert et al., 1995; Blakemore et al., 1999) and speech perception (Peelle, J. E. \& Sommers, 2015). In line with these findings, our results suggest that the sense of body ownership involves predictive cause-effect mechanisms that shape the processing of bodily signals during body-environment interactions. Notably, we show that such predictive mechanisms, which have the functional role of preserving and guiding the physical body through the environment (Graziano and Cooke, 2006), are operating likewise during body ownership illusions.

The modulation of visuotactile processing reported in this study relies probably on the same mechanisms for simultaneity constancy, which allows correct perception of truly synchronous crossmodal stimuli despite intrinsic differences in their processing latencies (Harrar and Harris, 2005; Kopinska and Harris, 2004). Mechanisms suggested for such temporal compensation, including sliding temporal binding windows (Sugita and Suzuki, 2003) or temporal ventriloquisms (Morein-Zamir et al., 2003), provide phenomenological descriptions but hardly insights into their neurophysiological basis. Although indirectly, our results provide support for a top-down modulation of multimodal neurons processing bodily signals (Graziano and Botvinick, 2002; Huang et al., 2012), and strongly indicate that such modulation can happen on the short time-scale of few tens of seconds.

In conclusion, our results provide experimental evidence that body ownership illusions affect multisensory integration. We have shown that, by establishing a causal link between the fake and physical bodies, the sense of body ownership enhances the temporal flexibility of visuotactile integration. This adds an important contribution to previous results on the sense of body ownership and ownership illusions. It was known that short periods of conflictive multisensory stimulations can induce dramatic illusory changes in our own-body representation, with important consequences at physiological (Moseley et al., 2008; Barnsley et al., 2011), psychological (Osimo et al., 2015) and behavioral (Peck et al., 2013; Banakou et al., 2013) levels. Here we show that changes occur also at the level of basic processing of multisensory information, which is relevant for the interaction of the body with the environment. Furthermore, these results demonstrate how the sense of body ownership guarantees a flexibility in visuotactile integration that goes beyond what is required in our normal daily experience where visual and tactile stimuli from the body are locked in space and time. 


\section{Materials and Methods}

\subsection{Participants}

Fourteen healthy subjects (8 female; mean age \pm SD: $20.7 \pm 2$ years) took part in Experiment 1 and another fourteen in Experiment 2 (8 female; mean age \pm SD: $21.6 \pm 4.6$ ). No participant had a history of neurological disease and all had normal or corrected-to-normal vision. They signed an informed consent form and received 10 euros as compensation. The experimental protocol was approved by the "Ethical Committee for research" of the University of Barcelona, in line with the institutional ethics and national standards for the protection of human participants.

\subsection{Experimental Setup}

Participants sat in front of table where a coin-vibrator was placed along the participant's sagittal plane (Fig. 1A).A wide field-of-view, stereo head-tracked, head-mounted display (HMD) was used to stream in 3D a virtual reproduction of the experimental room. By moving their head, participants could explore the environment. According to the experimental condition, when looking down towards their own body participants saw either a gender-matched virtual body from a first-personperspective and spatially coincident with their physical body, or two virtual sticks placed on the table. Participants' head and arms movements were tracked via a combination of infrared cameras and inertial devices.Vibrotactile stimulations were delivered via the coin-vibrator (Fig.1B) controlled through an Arduino board.

\subsubsection{Material and experimental Setup}

The virtual scenario was programmed with the Unity ${ }^{\circledR}$ software platform, using $3 D$ graphics elements designed with Autodesk $\circledast 3 \mathrm{ds}$ Max $\circledast$. The head mounted display has a $102^{\circ} \mathrm{H} \times 64^{\circ} \mathrm{V}$ total field-of-view, $1280 \times 1024$ resolution per eye and $60 \mathrm{~Hz}$ frame rate. The coin-vibrator used to deliver vibrotactile stimuli has the following specifics: $0.78 \mathrm{~cm}^{2}, 3 \mathrm{~V}, 100 \mathrm{~mA}, 50 \Omega, 1200 \pm 300$ r.p.m. The table was high enough $(94 \mathrm{~cm})$ to avoid participants having to bend their neck down during the temporal order judgment (TOJ) task (Figure 1A). The coin-vibrator was placed along the participant's sagittal plane at $24 \mathrm{~cm}$ from the table edge.

\subsubsection{System Calibration}

An oscilloscope (HAMEG Instruments) was used to measure the relative latencies of the visual and tactile target cues in the TOJ task, and to verify their effective duration (50 ms). When triggered at the same timeframe from the Unity® software script, the presentation of the visual cue on the HMD had a $30 \mathrm{~ms}$ time lag with respect to the onset of the vibration. This delay was compensated ad hoc in the Unity ${ }^{\circledR}$ script, so that the coded stimulus onset asynchrony (SOA) was precisely controlled.

\subsection{Temporal Order Judgment (TOJ) Task}

The TOJ task consisted in a forced choice discrimination of the temporal order of two target cues. 
The tactile one was a $50 \mathrm{~ms}$ long vibrotactile stimulus (single burst) delivered on the fingertip of the right index by a piezo-electric motor $(0.78 \mathrm{~cm} 2,1200 \pm 300$ r.p.m. $)$. The visual cue consisted in a $50 \mathrm{~ms}$ long rotation of a virtual geared-wheel object about its axis (Supplementary Video $\mathrm{S}^{2}{ }^{2}$ ). A complete session included 200 trials spanning a SOA range of $[-600,600]$, sampled at $\{ \pm 600$, $\pm 300, \pm 200, \pm 80\} \mathrm{ms}$. Each trial was presented $1200 \mathrm{~ms}$ after receiving the participant's response to the previous trial. The visual and tactile stimuli were spatially aligned in external space in all conditions but the No-Touch one in Experiment 1. In the latter case, the rotating wheel was seen slightly displaced from the virtual finger $(6 \mathrm{~mm})$, so that a small offset was introduced between the visual and tactile TOJ target-cues. This was implemented by slightly displacing the right virtual arm, during the TOJ, so that the tip of the virtual index was $6 \mathrm{~mm}$ away from the tip of the real index in the direction of the interphalangeal joint. During the task participants were instructed to keep their arms and head still, and fix their gaze on a blue dot displayed on the geared-wheel (Fig. 1C-E; Supplementary Video $\mathrm{S}^{2}{ }^{2}$. They used two pedals to provide responses.

\subsubsection{SOA SAMPLING}

The TOJ task consisted of 200 trials spanning a SOA range of $[-600,600] \mathrm{ms}$ and sampled at $\{ \pm 600$, $\pm 300, \pm 200, \pm 80\} \mathrm{ms}$, with associated sampling frequency of $\{10,20,30,40\}$. The SOA order was arranged in 10 cycles of 20 trials each; each cycle included all the sampled SOA values, one to four times according to their corresponding sampling frequency. Trials within each cycle were presented in a random order, independently for each subject and condition.

\subsection{Procedure}

Participants wore the HMD and headphones streaming white noise. First, they familiarized with the environment and performed a TOJ training session (25 trials). Next, they underwent two experimental sessions, counterbalanced across participants. The $200 \mathrm{TOJ}$ trials were presented in blocks of 25 trials, alternated with breaks in which participants were asked to mark with their hands the position of a cross appearing on the table at different positions (Video S2 ${ }^{2}$ ). In all conditions, but Stick in Experiment 2, the virtual body moved along with the participants tracked-movements. These breaks were included to make participants move their head and arms, so to relax tension and to keep high the sense of body ownership through congruent visuomotor correlations (Kokkinara and Slater, 2014). Each session had an average duration of 18 minutes.After its completion, participants filled a 5-item questionnaire customized to assess different aspects of the subjective illusory experience (Table 1).

\subsection{Analysis}

Questionnaire scores across conditions were compared with the Matched-pairs Wilcoxon tests, and the associated effect size was quantified in terms of "probability of superiority" of dependent measures, PS $_{\text {dep }}$, (Grissom and Kim, 2012).

2 Supplementary videos can be found at: https://www.nature.com/articles/srep30628\#-information 
TOJ responses, from each subject and condition, were converted into probabilities of "touch-first" response, $P\left(Y_{j}\right)$, at each SOA point tested. These values were next fitted with a psychometric curve of the form:

$P\left(Y_{j}\right)=\phi\left(\beta_{0}+\beta_{1} \times S O A\right)$

where $\phi($.$) denotes the probit function. The temporal window of integration was then estimated in$ terms of the Just Noticeable Difference (JND), by definition proportional to the inverse of the slope, $\beta_{1}$, and corresponding to the $75 \%$ threshold in the probability distribution (Moscatelli et al., 2012).

These fits provided the individual JND estimates adopted in the correlation analysis and used to generate the boxplots in Figs $2 A, B$ and $3 \mathrm{~A}$. Correlations among questionnaire scores and individual JND estimates were assessed in terms of Spearman rank coefficients.

At the group level, TOJ data were analyzed with a Generalized Linear Mixed Model (GLMM) that extends model (1) to include fixed effects associated to the experimental manipulation, and random effects associated to the variability within and between subjects (Agresti, 2002). The advantage of GLMMs with respect to group analysis based on parameters extraction from single subject fits, is that they take into account both inter and intra subject variability and have a higher statistical power (Agresti, 2002). Estimates of the JNDs and the associated $95 \% \mathrm{Cls}$ were computed with the bootstrap method (Agresti, 2002). Statistical analysis was performed in R. The GLMM analysis was performed using the glmer function from the Ime4 R package (Bates et al., 2015). 


\section{References}

Agresti,A. Categorial Data Analysis.Wiley-Interscience (John Wiley \& Sons, 2002).

Armel, K. C. \& Ramachandran, V. S. Projecting sensations to external objects: evidence from skin conductance response. Proc. Biol. Sci 270, 1499-506 (2003).

Banakou, D., Groten, R. \& Slater, M. Illusory ownership of a virtual child body causes overestimation of object sizes and implicit attitude changes. Proc. Natl. Acad. Sci. USA 110, 12846-12851 (2013).

Barnett-Cowan, M. \& Harris, L. R. Perceived timing of vestibular stimulation relative to touch, light and sound. Exp. Brain Res. 198, 221-31 (2009).

Barnsley, N. et al. The rubber hand illusion increases histamine reactivity in the real arm. Curr. Biol. 21, R945-R946 (2011)

Bates, D., Mächler, M., Bolker, B. \& Walker, S. Fitting Linear Mixed-Effects Models Using Ime4. J. Stat. Softw. 67, 1-48 (2015).

Bertamini, M. \& O'Sullivan, N. The use of realistic and mechanical hands in the rubber hand illusion, and the relationship to hemispheric differences. Conscious. Cogn. 27, 89-99 (2014).

Blakemore, S. J., Frith, C. D. \& Wolpert, D. M. Spatio-temporal prediction modulates the perception of self-produced stimuli. J. Cogn. Neurosci 11, 551-9 (1999).

Botvinick, M. \& Cohen, J. Rubber hands 'feel' touch that eyes see. Nature 391, 756 (1998).

Botvinick, M. Probing the Neural Basis of Body Ownership. Science 305, 782-783 (2004).

Corveleyn, X., López-Moliner, J. \& Coello, Y. Motor action reduces temporal asynchrony between perceived visual changes. J.Vis. 12, 20 (2012).

Ehrsson, H. H. In The New Handbook of Multisensory Processes (ed. Stein, B. E.) 775-792 (MIT Press, 2011).

Ehrsson, H. H., Spence, C. \& Passingham, R. E. That's my hand! Activity in premotor cortex reflects feeling of ownership of a limb. Science 305, 875-877 (2004).
Farmer, H., Tajadura-Jiménez, A. \& Tsakiris, M. Beyond the colour of my skin: How skin colour affects the sense of bodyownership. Conscious. Cogn. 21, 1242-1256 (2012).

Folegatti, A., Farnè, A., Salemme, R. \& de Vignemont, F. The Rubber Hand Illusion: two's a company, but three's a crowd. Conscious. Cogn. 21, 799-812 (2012).

Gallace, A. \& Spence, C. Visual capture of apparent limb position influences tactile temporal order judgments. Neurosci. Lett. 379, 63-68 (2005).

Gibson, J. J. In The Ecological Approach to Visual Perception 111-126 (Lawrence Erlbaum Associates, 1986).

Graziano, M. S. A. \& Cooke, D. F. Parieto-frontal interactions. personal space. and defensive behavior. Neuropsychologia 44, 845-859 (2006)

Grissom, R. J. \& Kim, J. J. Effect Sizes for Research: Univariate and Multivariate Applications 44, (Taylor and Francis Group, LLC, 2012).

Guterstam, A., Petkova, V. I. \& Ehrsson, H. H. The illusion of owning a third arm. PLoS One 6, e17208 (2011).

Haggard, P., Clark, S. \& Kalogeras, J. Voluntary action and conscious awareness. Nat. Neurosci. 5, 382-385 (2002).

Harrar, V. \& Harris, L. R. Simultaneity constancy: detecting events with touch and vision. Exp. brain Res. 166, 465-73 (2005).

Harris, L. R., Harrar, V., Jaekl, P. \& Kopinska, A. In Space and Time in Perception and Action (eds Nijhawan, R. \& Khurana, B.) 232-253 (Cambridge University Press, 2010).

Huang, R.-S., Chen, C., Tran, A. T., Holstein, K. L. \& Sereno, M. I. Mapping multisensory parietal face and body areas in humans. Proc. Natl. Acad. Sci. USA 109, 18114-9 (2012).

Keetels, M. \& Vroomen, J. In The Neural Bases of Multisensory Processes (eds Murray, M. \& Wallace, M.) 147-178 (CRC Press/Taylor \& Francis Group, 2012).

Kennett, S., Eimer, M., Spence, C. \& Driver, J. Tactile-visual links in exogenous spatial attention under different postures: convergent evidence from psychophysics and ERPs. J. Cogn. Neurosci 13, 462-478 (2001). 
Kilteni, K., Maselli,A., Koerding, K. \& Slater, M. Over my fake body: body ownership illusions for studying the multisensory basis of own-body perception. Front. Hum. Neurosci 9, doi: 10.3389/fnhum.2015.00141 (2015).

Kilteni, K., Normand, J.-M., Sanchez-Vives, M.V. \& Slater, M. Extending body space in immersive virtual reality: a very long arm illusion. PLoS One 7, e40867 (2012).

Kokkinara, E. \& Slater, M. Measuring the effects through time of the influence of visuomotor and visuotactile synchronous stimulation on a virtual body ownership illusion. Perception 43, 43-58 (2014).

Kopinska,A.\& Harris, L. R. Simultaneity constancy. Perception 33, 1049-1060 (2004).

Körding, K.P. et al. Causal inference in multisensory perception. PLoS One 2, e943 (2007).

Kuffler, S.W. Discharge Patterns and Functional Organization of Mammalian Retina. J. Neurophysiol. 16, 37-68 (1953).

Maister, L., Sebanz, N., Knoblich, G. \& Tsakiris, M. Experiencing ownership over a dark-skinned body reduces implicit racial bias. Cognition 128, 170-178 (2013).

Makin, T. R., Holmes, N. P. \& Ehrsson, H. H. On the other hand: dummy hands and peripersonal space. Behav. Brain Res. 191, 1-10 (2008).

Martini, M., Kilteni, K., Maselli, A. \& Sanchez-vives, M.V. The body fades away: investigating the effects of transparency of an embodied virtual body on pain threshold and body ownership. Sci. Rep 5, 13948 (2015).

Maselli, A. \& Slater, M. The building blocks of the full body ownership illusion. Front. Hum. Neurosci. 7, 83 (2013).

43. Maselli, A. \& Slater, M. Sliding perspectives: dissociating ownership from self-location during full body illusions in virtual reality. Front. Hum. Neurosci 8, 693 (2014).

Mégevand, P., Molholm, S., Nayak,A. \& Foxe, J. J. Recalibration of the Multisensory Temporal Window of Integration Results from Changing Task Demands. PLoS One 8, e71608 (2013).

Morein-Zamir, S., Soto-Faraco, S. \& Kingstone, A. Auditory capture of vision: examining temporal ventriloquism. Brain Res. Cogn. Brain Res. 17, 154-63 (2003).

Moscatelli, A., Mezzetti, M. \& Lacquaniti, F. Modeling psychophysical data at the population-level: The generalized linear mixed model. J.Vis. 12,1-17 (2012).

Moseley, G. L. et al. Psychologically induced cooling of a specific body part caused by the illusory ownership of an artificial counterpart. Proc. Natl.Acad. Sci. USA 105, 1316973 (2008).

Moutoussis, K. \& Zeki, S. A direct demonstration of perceptual asynchrony in vision. Proc. Biol. Sci 264, 393-399 (1997).

Newport, R., Pearce, R. \& Preston, C. Fake hands in action: embodiment and control of supernumerary limbs. Exp. brain Res. 204, 385-95 (2010).

Osimo, S. A., Pizarro, R., Spanlang, B. \& Slater, M. Conversations between self and self as Sigmund Freud-A virtual body ownership paradigm for self counselling. Sci. Rep 5, 13899 (2015).

Parise, C. V., Spence, C. \& Ernst, M. O. When correlation implies causation in multisensory integration. Curr. Biol. 22, 46-49 (2012).

Pavani, F. \& Zampini, M. The role of hand size in the fake-hand illusion paradigm. Perception 36, 1547-1554 (2007).

Peck, T. C., Seinfeld, S., Aglioti, S. M. \& Slater, M. Putting yourself in the skin of a black avatar reduces implicit racial bias. Conscious. Cogn. 22, 779-787 (2013).

Peelle, J. E. \& Sommers, M. S. Prediction and constraint in audiovisual speech perception. Cortex 68,169-181 (2015).

Petkova, V. I. \& Ehrsson, H. H. If I were you: perceptual illusion of body swapping. PLoS One 3, e3832 (2008).

Rochat, P. Self-perception and action in infancy. Exp. brain Res. 123, 102-9 (1998).

van Beers, R. J. \& Sittig, A. C. \& Denier Van Der Gon, J. J. The precision of proprioceptive position sense. Exp. Brain Res. 122, 367-377 (1998).

Samad, M., Chung, A. J. \& Shams, L. Perception of Body Ownership Is Driven by Bayesian Sensory Inference. PLoS One 10, e0117178 (2015).

Shams, L. \& Beierholm, U. R. Causal inference in perception. Trends Cogn. Sci. 14, 425-32 (2010).

Shore, D. I., Spence, C. \& Klein, R. M. Visual Prior Entry. 
Psychol. Sci. 12, 205-212 (2001).

Shore, D. I., Gray, K., Spry, E. \& Spence, C. Spatial modulation of tactile temporal-order judgments. Perception 34, 1251-1262 (2005).

Sugita, Y. \& Suzuki, Y. Implicit estimation of sound-arrival time. Nature 421, 27 (2003).

Tsakiris, M., Longo, M. R. \& Haggard, P. Having a body versus moving your body: Neural signatures of agency and bodyownership. Neuropsychologia 48, 2740-2749 (2010).

Tsakiris, M. My body in the brain: a neurocognitive model of body-ownership. Neuropsychologia 48, 703-12 (2010).32.

Vatakis, A. \& Spence, C. Crossmodal binding: evaluating the 'unity assumption' using audiovisual speech stimuli. Percept. Psychophys. 69, 744-756 (2007).

Tsakiris, M., Carpenter, L., James, D. \& Fotopoulou,A. Hands only illusion: multisensory integration elicits sense of ownership for body parts but not for non-corporeal objects. Exp. Brain Res. 204, 343-52 (2010).

van den Bos, E. \& Jeannerod, M. Sense of body and sense of action both contribute to self-recognition. Cognition 85, 17787 (2002).

van der Hoort, B., Guterstam, A. \& Ehrsson, H. H. Being Barbie: the size of one's own body determines the perceived size of the world. PLoS One 6, e20195 (2011).

Vatakis, A., Ghazanfar, A. a. \& Spence, C. Facilitation of multisensory integration by the 'unity effect' reveals that speech is special. J.Vis. 8, 14 (2008).

Vroomen, J. \& Keetels, M. Perception of intersensory synchrony: A tutorial review. Atten. Percept. Psychophys. 72, 871-884 (2010).

Wolpert, D. M., Ghahramani, Z. \& Jordan, M. I. An Internal Model for Sensorimotor Integration. Science 269, 1880-1882 (1995).

Yamamoto, S. \& Kitazawa, S. Sensation at the tips of invisible tools. Nat. Neurosci. 4, 979-980 (2001).

Zeller, D., Litvak, V., Friston, K. J. \& Classen, J. Sensory Processing and the Rubber Hand Illusion- An Evoked Potential Study. J. Cogn. Neurosci, doi: 10.1162/jocn (2014).
Zopf, R., Harris, J.A. \& Williams, M. A. The influence of bodyownership cues on tactile sensitivity. Cogn. Neurosci 2, 147154 (2011). 



\section{CHAPTER 4}

\section{The Body Fades Away:}

\section{INVESTIGATING THE EFFECTS OF TRANSPARENCY OF}

\section{AN EMBODIED VIRTUAL BODY ON PAIN THRESHOLD}

\section{AND BODY OWNERSHIP}

Related publication:

The body fades away : investigating the effects of transparency of an embodied virtual body on pain threshold and body ownership.

Martini, M., Kilteni, K., Maselli, A., and Sanchez-vives, M.V.

Sci. Rep. 5, 13948, 2015 


\section{Abstract}

The feeling of "ownership" over an external dummy/virtual body (or body part) has been proven to have both physiological and behavioural consequences. For instance, the vision of an "embodied" dummy or virtual body can modulate pain perception. However, the impact of partial or total invisibility of the body on physiology and behaviour has been hardly explored since it presents obvious difficulties in the real world. In this study we explored how body transparency affects both body ownership and pain threshold. By means of virtual reality, we presented healthy participants with a virtual co-located body with four different levels of transparency, while participants were tested for pain threshold by increasing ramps of heat stimulation. We found that the strength of the body ownership illusion decreases when the body gets more transparent. Nevertheless, in the conditions where the body was semi-transparent, higher levels of ownership over a seethrough body resulted in an increased pain sensitivity. Virtual body ownership can be used for the development of pain management interventions. However, we demonstrate that providing invisibility of the body does not increase pain threshold. Therefore, body transparency is not a good strategy to decrease pain in clinical contexts, yet this remains to be tested. 


\section{INTRODUCTION}

Visual feedback has been shown to effectively modulate pain sensations in experimental studies with both healthy participants and chronic pain patients (Martini et al., 2013; McCabe, 2002; Ramachandran and Altschuler, 2009; Hoffman et al., 2011). In particular, there is increasing evidence from pain studies showing how vision of the body may lead to effective pain relief (Longo et al. 2009; Mancini et al. 2011; Hegedüs et al., 2014; Martini et al., 2014; Romano and Maravita, 2014; Siedlecka et al., 2014). For instance, in a seminal study by Longo and co-workers, it was shown that the vision of one's hand produces analgesic effects compared to when the gaze is oriented toward an object or another's hand (Longo et al. 2009). In the same study the authors demonstrated that the attenuated behavioural response was paralleled by a significant decrease in the brain response elicited by the nociceptive stimuli. Further experimental research has confirmed that there is an effective interaction between the vision of the body and the sensation of pain. For example Mancini and co-workers demonstrated that the visual size of someone's own hand manipulated through mirrors leads to modulation of the pain threshold. In particular these authors reported that the vision of a smaller hand seen through a concave mirror leads to a higher pain threshold, while the vision of a bigger hand through a convex mirror decreases pain perception (Mancini et al., 2011). The rubber hand illusion provided researcher with a paradigm to experimentally induce illusory ownership of external body parts, so that the fake limb is felt as belonging to one's body (Botvinick and Cohen, 1998). However, there are contradictory findings regarding whether looking at an owned rubber hand is analgesic or not. Recently, it has been reported that the analgesic effect of looking at one's own hand is not present when looking at an owned rubber hand (Mohan et al., 2012). However, a subsequent study using a similar paradigm found the opposite results, with an effect comparable to that obtained by the vision of the real limb (Hegedüs et al., 2014). Moreover, further experimental evidence has shown how the analgesic effect of looking at one's own body is present also when the painfully-stimulated volunteer is looking at an avatar's limb, provided that this is felt as her/his own(Martini et al. 2014). Furthermore, the vision of dynamic colour changes applied to the skin of the owned avatar's limb modulates pain threshold with heat stimuli such that a reddish skin colour leads to lower threshold compared to the vision of a bluish one (Martini et al., 2013). Hansel and co-workers reported that during an out-of-body experience, an increase in the self-identification with an avatar's body seen in front of the participant is associated with an increase in pressure pain threshold (Hänsel et al., 2011). Lately, following a similar paradigm, Romano and colleagues reported a reduced skin conductance response to painful stimuli when participants saw and identified themselves with the avatar body displayed in front of them (Romano et al., 2014). All of these studies provide interesting insights on how the vision of the body can play a major role in the modulation of pain perception.

Body-related visual feedback is known to produce analgesic or pain relief effects in clinical populations as well.The experience of a phantom limb, typically reported by amputees, is often accompanied by painful sensations that are elicited or exacerbated by different physical and psychological factors (Flor, 2002). Phantom limb pain has been treated by means of mirror therapy, where the patient can see an intact limb at the location where the phantom limb is felt to be, thus providing an illusory visual feedback that the lost limb is still there (Ramachandran and Altschuler, 2009). Also, it has been 
shown that viewing one's painful limb as becoming smaller decreases the pain related to that limb (Moseley et al., 2008). The same manipulation though has been shown to lead to the opposite effect in healthy participants (Mancini et al., 2011), probably due to the different clinical conditions.

While the effects related to the vision of the body are classically related to dichotomous body versus no-body paradigms, namely to seeing or not seeing the body part, it remains unknown whether and how different levels of visibility of the body could affect pain perception. One could hypothesize that the vision of a body that fades away could increase analgesia by perceiving one's own body as less likely to be harmed by the painful stimulus because it is fading away, or, conversely decrease analgesia due to the analgesic effect of the vision of the body (Longo et al., 2008; Hegedüs et al. , 2014; Martini et al., 2014). Therefore an investigation on the effects of body transparency is needed. However, from a technical point of view, rendering a visible object "transparent" or "semi-transparent" is not trivial. For instance, paradigms relying on visual masking (Breitmeyer and Ogmen, 2000) or on binocular rivalry (Arnold et al., 2007) provide only transient illusions and do not really offer valid solutions to make solid, non-transparent matter invisible. Also, these techniques usually use simple visual cues rather than complex physical entities such as human bodies. Immersive Virtual Reality (IVR) technology represents an effective tool as it allows the creation of sensory environments that can be replicated almost identically and that are under the full control of the experimenter (Sanchez-Vives and Slater, 2005). Furthermore, not only does IVR allow one to feel immersed and present in a computer-generated environment, but it also makes possible the induction of the illusion of owning a virtual body (Sanchez-Vives and Slater, 2005; Kilteni et al., 2012), a body that can be rendered with the morphological characteristics that the experimenter determines (Banakou et al., 2013). This illusion has been shown to affect the perceptual responses of participants (Llobera et al., 2013). So, thanks to its unique advantage to manipulate visual information in a controlled and systematic manner, IVR represents an excellent tool for investigating body-related human perception, including pain perception, under both healthy and clinical conditions.

Here we aimed at investigating whether and how much the illusion of body ownership over a virtual body and pain threshold change as the body becomes increasingly more transparent. We presented healthy participants with a co-located virtual body with four different levels of transparency, while participants were tested for pain threshold by increasing ramps of heat stimulation.

\section{Results}

The experiment comprised different visual conditions where participants could see the avatar's body from a first person perspective with different degrees of transparency (see Fig. 1). In particular, this within group design entailed a single factor, "transparency", with 4 different levels $(0 \%, 25 \%$, $50 \%, 75 \%$ of transparency). The order of the conditions was counterbalanced between participants. Three heat ramps were provided for each condition, where participants were instructed to stop the stimulation as soon as they felt the heat to be painful.After each heat stimulation participants were also asked to rate the level of the illusion of ownership over the virtual limb (for further details see 
Methods section).
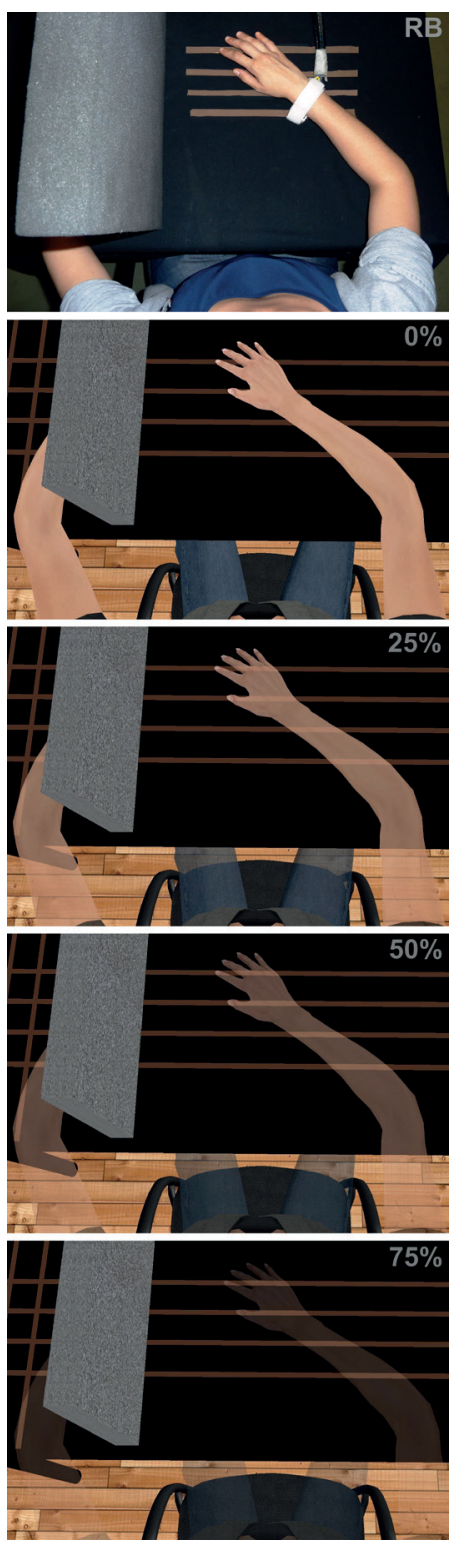

Figure 1:The experimental set-up (i.e. real body, RB) and the experimental conditions. From top to bottom, the avatar's body is rendered fully opaque (0\% transparency), or with $25 \%, 50 \%$ and $75 \%$ of transparency. The exemplifying picture was taken by MM and the person depicted is KK (both authors of the present manuscript). The virtual environment was programmed using the XVR system (VRMedia S.r.l., Pontedera, Italy; Tecchia et al., 2010, ) and the virtual body using the HALCA library (Gillies, M. and Spanlang, 2010) 


\section{DEPENDENCY OF OWNERSHIP ON OPACITY/TRANSPARENCY OF THE VIRTUAL ARM}

The reported levels of ownership showed a clear dependence on the transparency levels, being ownership lower with higher transparency (Fig.2). Indeed we found a significant negative correlation (Spearman correlation: $r_{s}=-0.32, p=0.0017$ ) between the two. Thus, in these conditions, the higher the level of transparency the lower the sense of ownership.An ordered logistic regression revealed that the factor "transparency" (treated as a numeric variable) was a significant predictor of "ownership" (treated as an ordered categorical variable), which was fit with a negative coefficient $(c=-0.52)$ at a confidence level $p=0.0016$. A Friedman One-Way Anova was then run to check whether there were different body ownership levels among the four conditions. This test showed that indeed the body ownership levels were significantly different under the diverse transparency conditions $\left(\chi^{2}=15.87 ; p=0.001\right)$. Single comparisons were run with matched pairs Wilcoxon tests. Together with the $p$-value we report the effect size in terms of the probability of superiority of dependent measures $\left(\mathrm{PS}_{\mathrm{dep}}\right)$ : in the most transparent condition $(75 \%)$ ownership was significantly lower than in the $50 \%\left(z=-2.57, p=0.01, \mathrm{PS}_{\text {dep }}=0.79\right), 25 \%\left(z=-2.97, p=0.003, \mathrm{PS}_{\text {dep }}=0.79\right)$ and $0 \%\left(z=-3.1, p=0.002\right.$, PS $\left._{\text {dep }}=0.82\right)$ transparency conditions (see method sections for more details on how different levels of transparency were implemented); in the $50 \%$ condition ownership was significantly lower than in the $0 \%$ condition $\left(z=-1.97, p=0.048, P_{\text {dep }}=0.68\right)$; the rest of comparisons resulted in no significant difference ( $p=0.13$ for $25 \%$ vs $0 \%$ and $p=0.16$ for $50 \%$ vs $25 \%$ ), showing nevertheless a trend for lower ownership scores in the higher transparency condition.

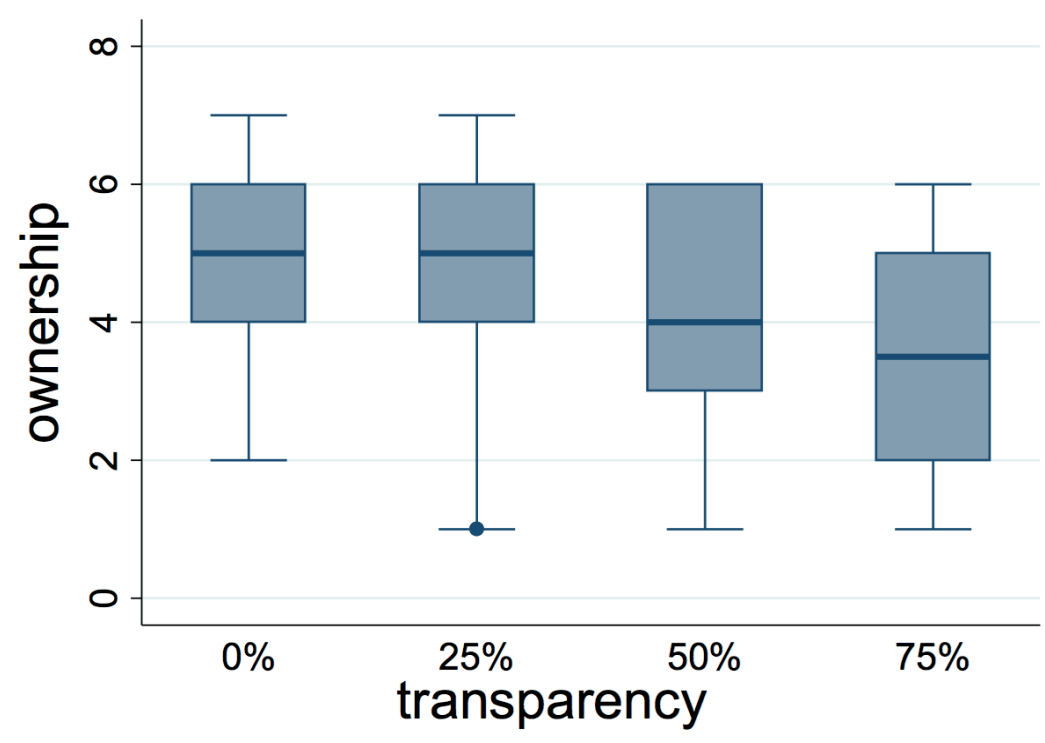

Figure 2:The box-and-whisker plot shows levels of ownership of the avatar's body according to the different levels of body transparency (from $0 \%=$ fully opaque to $75 \%$ of transparency). 


\section{DEPENDENCY OF PAIN THRESHOLD ON OPACITY/TRANSPARENCY OF THE VIRTUAL ARM}

The data from our experiment show no direct dependence of the pain threshold (PT) on the transparency of the virtual body. In fact, a repeated measure one-way ANOVA with factor "transparency" gave no significant results $\left(F_{(3,23)}=0.87, p=0.46\right)$. Analogous results were found when including the temporal order of conditions as a covariate (ANCOVA), which would account for habituation effects (transparency: $F_{(3,23)}=1.59, p=0.2$; block: $F_{(3,23)}=57.9, p<0.0001$ ). The latter were nevertheless taken into account in the experimental design via the perfect balance in the order presentation of the four experimental conditions.

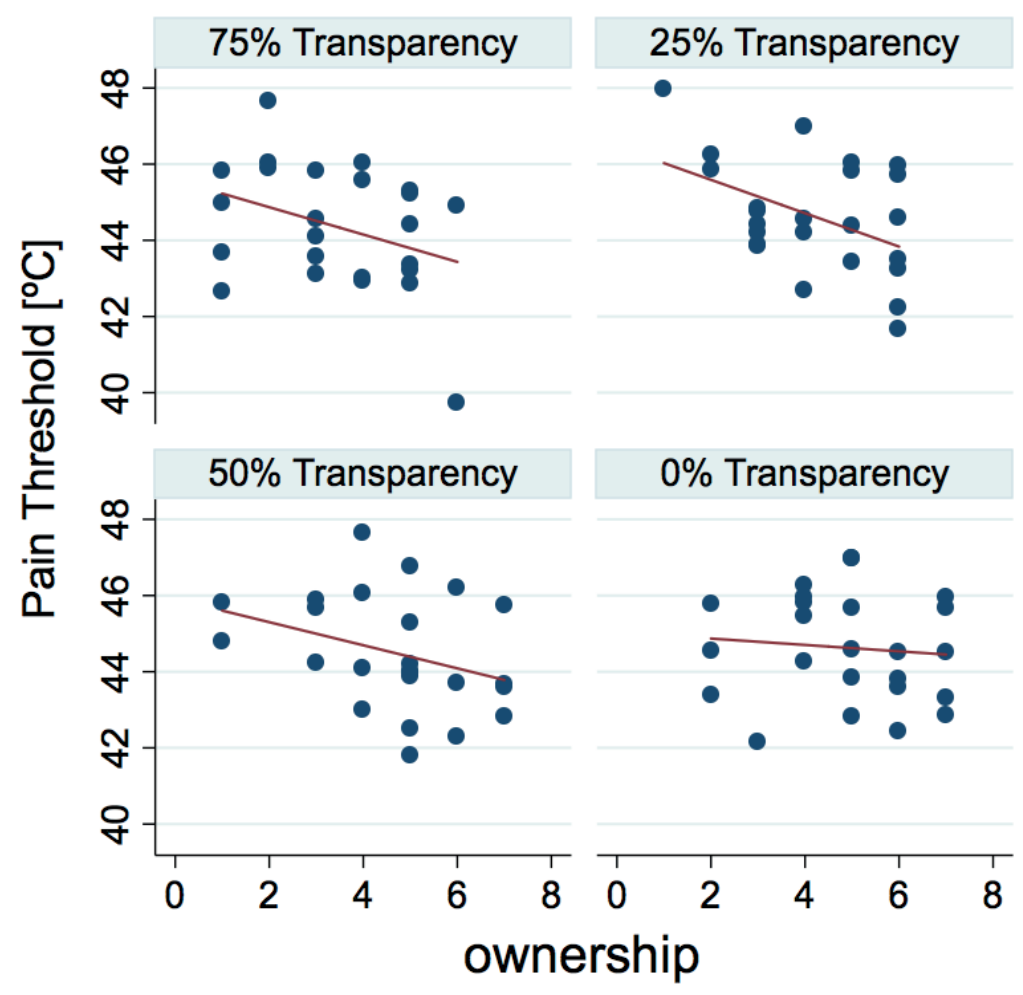

Figure 3: Scatterplots of ownership levels and pain threshold per participant and condition. Trend-lines show a trend or significant negative correlations only for the semi-transparent bodies (25\%,50\% and $75 \%)$.

Interestingly, the data showed a negative correlation of PT with the level of ownership (Spearman correlation: $\left.r_{s}=-0.27, p=0.007\right)$; note that higher PT values indicate a higher tolerance to painful stimuli, so this result suggests that the higher the sense of ownership the less the tolerance to 
painful stimuli. However, including all conditions together such correlation could be plagued by the autocorrelation bias. So, we ran the correlation separately for each level of transparency, and we found: $\left[r_{s}=-0.30, p=0.152\right]$ for $75 \%$ transparency; $\left[r_{s}=-0.39, p=0.064\right]$ for $50 \%$ transparency; $\left[r_{s}=-0.42, p=0.04\right]$ for $25 \%$ transparency; $\left[r_{s}=-0.1, p=0.46\right]$ for $0 \%$ transparency. Therefore, we found that the negative correlation between PT and ownership holds (i.e. is significant or in trend) only for the conditions with a transparent body $(75 \%, 50 \%, 25 \%)$. No negative correlation is found for the case of a normal, fully opaque, virtual body (Fig. 3). Putting together data from the three conditions with a transparent body we found a significant negative correlation with PT $\left[r_{s}=-0.33\right.$, $p=0.005]$, meaning that the higher the ownership of the transparent virtual arm, the lower the PT.

Not taking into account the level of ownership, PT shows no relationship with transparency (Spearman correlation: $r_{s}=-0.03, p=0.74$ ).

\section{Discussion}

Motivated by gathering new insights into the effects of visual exposure to the body and pain perception, and in an attempt to find a novel visual mechanism capable of modulating pain, in the present experiment we changed the virtual body visibility by making the avatar's body progressively transparent. To our knowledge, this is the first study that addresses the influence of body visibility on both the sense of ownership and its impact on pain perception.

The results obtained from our set-up suggest that while the illusion of ownership is significantly reduced by increasing the transparency of the virtual body, pain threshold does not seem to be directly modulated by it. On the other hand, significant negative correlations were found between pain threshold and the subjective level of ownership of the transparent body. More specifically, this relation was found to hold for all the conditions in which participants were in view of a transparent body, but not when staring at a fully opaque virtual body. In other words, experiencing a greater sense of ownership over a transparent body results in a diminished pain threshold, and so in a higher sensitivity to painful stimuli. Importantly, our results are compatible with previous studies showing that the vision of one's own body part (either real, dummy, or virtual) leads to analgesic effects (Longo et al., 2008; Hegedüs et al, 2014; Martini et al., 20014 respectively). Indeed there is no negative relationship between body ownership and pain threshold when the displayed body is normally displayed ( $0 \%$ transparency).

There are various possible interpretations for the negative relationship between pain and body transparency. First, it can be argued that a transparent body can imply some sort of weakness or vulnerability and therefore it can be perceived as more sensitive and susceptible to be hurt. In agreement with this argument, a recent study demonstrated that the illusion of owning a marble hand, induced by replacing the auditory impact of a hit on the participants' hand with the sound of a hammer against a piece of marble, people perceived their hand to be harder and less sensitive (Senna et al., 2014). Further, Osumi and coworkers recently reported that when participants are painfully stimulated while they experience the illusion of owning a rubber hand that is injured (i.e. 
presenting a bloody scar along the rubber limb), their pain threshold decreases (Osumi et al., 2014a).

In addition, owning a transparent body may lead to general negative feelings toward the body and consequently, this attitude may influence the pain perception negatively. In agreement with this explanation, a recent study showed how unpleasant emotions toward a modified (specifically: magnified) image of one's hand are related to lower pain thresholds (Osumi et al., 2014b). Interestingly, in this study, no association with a lower pain threshold was found when the limb was not visually modified, which is in accordance with our findings. With an alternative interpretation we speculate that transparency may result in blurring the body limits, thus decreasing the predictability for potential injuries; hence, being uncertain about the body boundaries, the brain proceeds in a general lowering of the pain threshold as an alert system, strengthening the protective mechanisms.

Our results could also be complementary to those showing that seeing one's own body produces analgesia and that this effect vanishes when the visual cue is something else, namely another's body or an object (Longo et al., 2008; Martini et al., 2014). The neural underpinnings of this effect are associated to reduced activations of key areas of the putative 'pain matrix' during the vision of the body (Longo et al., 2012). Indeed, studies with chronic pain patients and healthy participants point at the intracortical excitability in the primary somatosensory cortex as the mechanism underlying pain modulation (Longo et al., 2012). The vision of the body would not only increase the intracortical inhibition within somatosensory cortex but it would also facilitate a reorganization of its somatosensory maps sharpening the receptive fields of the neurones (Haggard et al., 2013). These two mechanisms have been proposed to underlie the analgesia that follows the vision of the body, an effect that vanishes in the absence of such visual cue. In line with these proposed mechanisms, we found that there is a relationship between owning a virtual body that gets transparent and the loss of the analgesic effect derived from seeing one's own body (Longo et al., 2008).

In addition, our results reveal that the ownership of a virtual arm by means of co-location decays with the transparency of the arm; in other words, ownership of an opaque arm is significantly higher than that of a semi-transparent arm. This result is in agreement with several findings in the literature on body ownership illusions that emphasize the role of top-down influences in modulating the level of illusion, in terms for example of body volume (IJsselsteijn et al., 2006), body length (Kilteni et al., 2012), or body connectivity (Perez-Marcos et al., 2011). Our results of reduced body ownership scores toward a semi-transparent arm may apparently disagree with the results by Guterstam and coworkers who recently demonstrated that people can experience strong sensations of body ownership toward an empty area of the space when congruent visuotactile correlations are provided to the participant's limb and the empty area (Guterstam et al., 2013). However, any comparison between the two studies should be done with caution due to significant methodological differences; for example, in Guterstam's study, there was no body part displayed, while in our experiment a body was always shown under different transparency levels. In addition, these authors provided visuotactile stimulation in order to induce the "ownership" of the empty area, while in our study the first person perspective of a collocated avatar's body was sufficient to achieve the virtual body illusion (Maselli and Slater, 2013; Tieri et al., 2015). We did not provide additional visuotactile stimulation in order to avoid ceiling effects on the level of the ownership illusion, which might have hindered the detection of modulations effects associated to different levels of transparency. 


\section{Conclusion}

A number of studies have been conducted to explore how bodily illusions may affect our perception. However, how body visibility affects body ownership and pain processing is new since it is an effect hard to achieve in the real world. Such approach not only expands our knowledge about how we perceive our body and interact with it and the environment, but it can also be a critical tool for the development of pain management interventions relevant for clinical therapy (Ramakonar et al., 2011). In the present study we found that the vision of a transparent body may lead to lower levels of ownership over that body. Participants reporting stronger ownership over the transparent body showed lower levels of pain threshold. Therefore, although visual transformations of the owned virtual body can lead to useful applications in therapeutical settings, making the body transparent does not seem to be useful to increase the pain threshold. From these observations we could predict that if a patient reports pain in a limb, making this limb (virtually) transparent would not decrease his/her pain. However, such hypothesis remains to be confirmed by future studies. Here we studied pain threshold on healthy subjects, however chronic pain has additional emotional and psychological components, and therefore the transparency of the painful limb could have a different impact in this clinical population.

\section{Methods}

\section{1 Participants}

24 right-handed female healthy participants (age: $21.1 \pm 1.8$ ) were recruited for the experiment. We intentionally chose participants of the same gender given that men and women adapt differently (sensory habituation) to pain stimuli. All participants had normal or corrected-to-normal vision and no history of neurological or psychological disorders.Also, any condition potentially interfering with pain sensitivity (e.g. drug intake) was considered as a further exclusion criterion. Upon arrival at the laboratory they were asked to read and sign a consent form and the experiment was carried out in accordance with the regulations of the local ethics committee (Comité Ético de Investigación Clínica de la Corporación Sanitaria Hospital Clínic de Barcelona) and with the declaration of Helsinki. The study was approved by the ethics committee of the Hospital Clinic of Barcelona. All participants received a monetary compensation for their participation (10€).

\subsection{VIRTUAL REALITY SYSTEM}

The stereoscopic head-mounted display (HMD) was a NVIS SX111 with a resolution of $1280 \times 1024$ per eye and a total field of view of $102^{\circ} \times 64^{\circ}$, displayed at $60 \mathrm{~Hz}$. The head-tracking was realized with a 6-DOF Intersense IS-900 device (InterSense, Billerica, USA). The virtual environment was implemented using the XVR system (Tecchia et al., 2010. VRMedia S.r.l., Pontedera, Italy) and the virtual body was displayed using the HALCA library (Gillies and Spanlang, 2010). Transparency of the virtual body was achieved by adjusting the value of alpha channel of the texture of the virtual body to levels of either $100 \%$ (maximum opacity), $75 \%, 50 \%$ or $25 \%$ (very transparent) (see Fig. 1). 
Noise isolation was ensured by the administration of pink noise through a surround audio system (Creative technology Ltd., Singapore), with a constant volume set at $65 \mathrm{~dB}$.

\subsection{Pain threshold measurement}

Thermal heat stimuli were delivered by means of a Somedic-Thermotest machine (Somedic, Stockholm, Sweden) with a $2.5 \mathrm{~cm} \times 5.0 \mathrm{~cm}$ thermode tied with a Velcro strap on the front of the participant's right forearm, close to the distal extreme of the radius (see Fig. 1). Pain thresholds were assessed with the method of limits (Yarnitsky et al., 1995). The probe temperature was increased from normal skin temperature (constant baseline temperature $=31^{\circ} \mathrm{C}$ ) at $2{ }^{\circ} \mathrm{C} / \mathrm{s}$. The temperature stimulation was provided in a continuous fashion, gradually and constantly increasing from the baseline temperature till the subjective pain threshold level. Participants were instructed to press a button with their left hand as soon as they perceived the stimulation on their right hand as being painful. Immediately after pushing the kill-switch button, the probe temperature rapidly decreased to the baseline temperature. For safety reasons, maximal temperature was set at $48^{\circ} \mathrm{C}$.

\subsection{Procedure}

Participants sat comfortably on a chair with both arms resting on a table covered with a black cloth as shown in Fig. 1. Before starting the main part of the experiment, participants were given 2-3 heat stimuli to familiarize them with the heat ramps. Afterwards, the participant put on the HMD, the room's lights were turned off and the pink noise played. The posture of the participant was calibrated in order to ensure a close co-location of the real body with the virtual one. The HMD allowed participants to experience an immersive virtual environment around them and to see a virtual body from a first-person perspective so that, when they looked down towards their own body, they could see the virtual body in place of their own. Participants were asked to keep still throughout the experiment. Importantly, no other sensory correlations were provided (visuo-tactile or visuo-motor correspondences) since a first person perspective over a realistic fake humanoid body has been shown to be sufficient in eliciting a strong body ownership illusion (Maselli and Slater, 2013). Four brown stripes of tape were stuck on the real table and displayed in the virtual table to facilitate the perception of a transparency of the avatar's arm and to boost the visuo-tactile correspondence between the real and the virtual hand (both the real and the virtual hand touching the first stripe with the finger and the second stripe with the thumb).

Before each condition, participants were given approximately one minute to familiarize with the virtual room in which they were in and with the virtual body. The experiment consisted of four different conditions, presented in blocks, with the avatar's body transparency set at $0 \%, 25 \%, 50 \%$ or $75 \%$. All participants completed the four conditions, with the order of the blocks balanced across participants. Each condition started with the participant looking at and describing the entire body and then focussing on the avatar's right wrist (i.e. where the painful stimuli came from). The inter-stimulus interval was set at around 40 seconds and three heat ramps were provided for each condition.

Within each trial, about 2 seconds after the thermal stimulation had been stopped, a "beep" sound prompted the participants to judge the level of ownership over the virtual limb. They were instructed to verbally report a number on a scale from 1 to 7 (" 1 " = not at all," 7 " completely) to reply to the 
question: "Did you feel as if the virtual right arm was your own right arm?". Participant's ratings were promptly annotated by the experimenter. 


\section{References}

Arnold, D. H., Grove, P. M. \& Wallis, T. S. A. Staying focused: I See Before Me? The Rubber Hand Illusion in Reality, Virtual a functional account of perceptual suppression during binocular Reality, and Mixed Reality. Presence Teleoperators Virtual rivalry. J.Vis. 7, 7.1-8 (2007). Environ. 15, 455-464 (2006).

Banakou, D., Groten, R. \& Slater, M. Illusory ownership of Kilteni, K., Groten, R. \& Slater, M. The Sense of Embodiment a virtual child body causes overestimation of object sizes and in Virtual Reality. Presence Teleoperators Virtual Environ. 21, implicit attitude changes. Proc. Natl. Acad. Sci. USA 110, 373-387 (2012).

12846-51 (2013).

Kilteni, K., Normand, J.-M., Sanchez-Vives, M.V. \& Slater, M. Botvinick, M. \& Cohen, J. Rubber hands 'feel' touch that eyes Extending body space in immersive virtual reality: a very long see. Nature 391, 756 (1998). arm illusion. PLoS One 7, e40867 (2012).

Breitmeyer, B. G. \& Ogmen, H. Recent models and findings Kilteni, K., Maselli, A., Kording, K. P. \& Slater, M. Over my fake in visual backward masking: A comparison, review, and update. body: body ownership illusions for studying the multisensory basis Percept. Psychophys. 62, 1572-1595 (2000). of own-body perception. Front. Hum. Neurosci. 9, 141 (2015).

Flor, H. Phantom-limb pain:characteristics, causes, and treatment. Llobera, J., Sanchez-Vives, M. V. \& Slater, M. The relationship Lancet Neurol. 1,182-189 (2002). between virtual body ownership and temperature sensitivity. J. R. Soc. Interface 10, 20130300 (2013).

Gillies, M. \& Spanlang, B. Comparing and evaluating real time character engines for virtual environments. Presence Longo, M. R., Betti, V., Aglioti, S. M. \& Haggard, P. Visually Teleoperators Virtual Environ. 19, 95-117 (2010). $\quad$ induced analgesia: seeing the body reduces pain. J. Neurosci. Off. J. Soc. Neurosci. 29, 12125-12130 (2009).

Guterstam, A., Gentile, G. \& Ehrsson, H. H. The invisible hand illusion: multisensory integration leads to the embodiment of a Longo, M. R., lannetti, G. D., Mancini, F., Driver, J. \& Haggard, discrete volume of empty space. J. Cogn. Neurosci. 25, 1078- P. Linking pain and the body: neural correlates of visually induced 99 (2013) analgesia. J. Neurosci. 32, 2601-7 (2012).

Haggard, P., lannetti, G. D. \& Longo, M. R. Spatial sensory Mancini, F., Longo, M. R., Kammers, M. P. M. \& Haggard, P. organization and body representation in pain perception. Curr. Visual distortion of body size modulates pain perception. Psychol. Biol. 23, R164-76 (2013). Sci. 22, 325-30 (2011).

Hashmi, J. A. \& Davis, K. D. Effects of temperature on heat Maselli, A. \& Slater, M. The building blocks of the full body pain adaptation and habituation in men and women. Pain 151, ownership illusion. Front. Hum. Neurosci. 7, 83 (2013). 737-43 (2010).

Martini, M., Perez-Marcos, D. \& Sanchez-Vives, M. V. What Hänsel, A., Lenggenhager, B., von Känel, R., Curatolo, M. \& Color is My Arm? Changes in Skin Color of an Embodied Virtual Blanke, O. Seeing and identifying with a virtual body decreases Arm Modulates Pain Threshold. Front. Hum. Neurosci. 7, 438 pain perception. Eur. J. Pain 15, 874-9 (2011).

(2013)

Hegedüs, G. et al. The rubber hand illusion increases heat Martini, M., Perez-Marcos, D. \& Sanchez-Vives, M. V. pain threshold. Eur. J. Pain (2014). doi: 10.1002/j.1532- Modulation of pain threshold by virtual body ownership. Eur. J. 2149.2014.00466.x Pain (2014). doi: 10.1002/j.1532-2149.2014.00451.x.

Hoffman, H. G. et al. Virtual reality as an adjunctive non- McCabe, C. S. A controlled pilot study of the utility of mirror pharmacologic analgesic for acute burn pain during medical visual feedback in the treatment of complex regional pain procedures. Ann. Behav. Med. a Publ. Soc. Behav. Med. 41, syndrome (type 1). Rheumatology 42, $97-101$ (2002). 183-191 (2011).

Mohan, R. et al. No Pain Relief with the Rubber Hand Illusion. IJsselsteijn,W.A., de Kort,Y.A.W. \& Haans,A. Is This My Hand PLoS One 7, e52400 (2012 
$1247-59(2015)$

Moseley, G. L., Parsons, T. J. \& Spence, C. Visual distortion of a limb modulates the pain and swelling evoked by movement. Yarnitsky, D., Sprecher, E., Zaslansky, R. \& Hemli, J. A. Heat Curr. Biol. CB 18, R1047-1048 (2008). pain thresholds: normative data and repeatability. Pain 60,32932 (1995).

Osumi, M., Imai, R., Ueta, K., Nobusako, S. \& Morioka, S. Negative body image associated with changes in the visual body appearance increases pain perception. PLoS One 9, e107376 (2014a).

Osumi, M. et al. Factors associated with the modulation of pain by visual distortion of body size. Front. Hum. Neurosci. 8, 137 (2014b).

Perez-Marcos, D., Sanchez-Vives, M.V. \& Slater, M. Is my hand connected to my body? The impact of body continuity and arm alignment on the virtual hand illusion. Cogn. Neurodyn. 6, 295-305 (2011).

Ramachandran, V. S. \& Altschuler, E. L. The use of visual feedback, in particular mirror visual feedback, in restoring brain function. Brain 132, 1693-710 (2009).

Ramakonar, H., Franz, E. A. \& Lind, C. R. P. The rubber hand illusion and its application to clinical neuroscience. J. Clin. Neurosci. 18, 1596-601 (2011).

Romano, D., Pfeiffer, C., Maravita, A. \& Blanke, O. Illusory self-identification with an avatar reduces arousal responses to painful stimuli. Behav. Brain Res. 261, 275-81 (2014).

Romano, D. \& Maravita, A. The visual size of one,s own hand modulates pain anticipation and perception. Neuropsychologia 57, 93-100 (2014).

Sanchez-Vives, M.V.\& Slater, M. From presence to consciousness through virtual reality. Nat. Rev. Neurosci. 6, 332-339 (2005).

Senna, I., Maravita, A., Bolognini, N. \& Parise, C. V. The Marble-Hand Illusion. PLoS One 9, e91688 (2014).

Siedlecka, M., Klimza, A., Łukowska, M. \& Wierzchon, M. Rubber hand illusion reduces discomfort caused by cold stimulus. PLoS One 9, e109909 (2014).

Tecchia, F. et al. A Flexible Framework for Wide Spectrum VR Development. Presence Teleoperators Virtual Environ. 19, 302312 (2010).

Tieri, G., Tidoni, E., Pavone, E. F. \& Aglioti, S. M. Mere observation of body discontinuity affects perceived ownership and vicarious agency over a virtual hand. Exp. brain Res. 233, 



\section{CHAPTER 5}

\section{Over Mr Fake Body:}

\section{BODY OWNERSHIP ILLUSIONS FOR STUDYING THE}

\section{MULTISENSORY BASIS OF OWN-BODY PERCEPTION}

Related publication:

Over my fake body: body ownership illusions for studying the multisensory basis of own-body perception. Kilteni*, K., Maselli*,A., Kording, K.P., and Slater, M.

Front. Hum. Neurosci. 9:141, 2015

* Equal contribution 


\section{Abstract}

Which is my body and how do I distinguish it from the bodies of others, or from objects in the surrounding environment? The perception of our own body and more particularly our sense of body ownership is taken for granted. Nevertheless, experimental findings from body ownership illusions (BOls), show that under specific multisensory conditions, we can experience artificial body parts or fake bodies as our own body parts or body, respectively. The aim of the present paper is to discuss how and why BOls are induced. We review several experimental findings concerning the spatial, temporal, and semantic principles of crossmodal stimuli that have been applied to induce BOls. On the basis of these principles, we discuss theoretical approaches concerning the underlying mechanism of BOls. We propose a conceptualization based on Bayesian causal inference for addressing how our nervous system could infer whether an object belongs to our own body, using multisensory, sensorimotor, and semantic information, and we discuss how this can account for several experimental findings. Finally, we point to neural network models as an implementational framework within which the computational problem behind $\mathrm{BO}$ s could be addressed in the future. 


\section{INTRODUCTION}

"I swear to God, cross my heart, I haven't (been kidding). A man should know his own body, what's his and what's not—but this leg, this thing.... doesn't feel right, doesn't feel real一and it doesn't look part of me" (Sacks, 1985).

There would be nothing wrong or weird with the above statement, if we thought that the person speaking had been referring to a fake leg, which for e unknown reason was placed close to his body. However, in fact the quote is from a brain-damaged patient talking with his doctor about his own paralyzed leg. The bizarre neurological syndrome of somatoparaphrenia reveals that our ability to recognize our own body parts can dramatically deteriorate in certain brain damage conditions (Vallar and Ronchi, 2009; Feinberg et al., 2010).Astonishing as it is, the case of somatoparaphrenia gives prominence to the multidisciplinary research topic (Gallagher, 2000; Jeannerod, 2003; Blanke and Metzinger, 2009; de Vignemont, 2011) that aims to address a seemingly trivial question: how does our brain distinguish between our own body and those of other people or objects?

A key difference between the perception of our own body and that of others' bodies or objects in the environment lies in the type of sensory input available to the brain. In processing our own body, the brain has access to a set of sensory information - such as somatosensation, thermosensation, nociception, interoception, and vestibular signals - that is not available for the perception of other objects or bodies. Yet, evidence from somatoparaphrenic patients suggests that the intact sensory processing from separate modalities may not be in itself sufficient for the emergence of the feeling of body ownership. Indeed, it has been proposed that somatoparaphrenia may be due to an impairment in processing multisensory signals (Vallar and Ronchi, 2009). Therefore, the sense of body ownership should be regarded as the outcome of the brain's processes that integrate different sensory cues into the unified perception of "my body."

Another key difference concerns the fact that in own-body perception all the sensory and motor cues that converge into the "my body" percept are strictly bound by physical laws. For example, when striking a fist on a table, the view of the contact is always accompanied by the punching tactile sensation in our hand. This is not the case for the perception of external objects or others' bodies that, although multisensory in nature, is not subject to such strict constraints. Just consider this example: you see a dog in a park and you hear a barking sound at about the same moment and coming from the same direction. Even if it is probable that it was the seen dog that was barking, there is also the possibility that there is a second dog just behind the tree. Thus, while in the perception of external events we can contemplate the possibility of different sources, in the perception of "my body" this is not the case, since all multimodal cues involved originate from the same source: the physical body.

Due to the fact that the body-related multimodal cues are tightly bound together and not independent, it is difficult to experimentally investigate body ownership with the methods adopted in multisensory research. In contrast, when studying the multisensory perception of external objects, including others' bodies, experiments typically involve the concurrent presentation of sensory 
signals that are independent. This permits the introduction of delays between the occurrences of the stimuli, to present them from different positions, or even to manipulate their information content so that these may refer to the same context or not. Therefore, it is possible to investigate how multisensory perception is influenced by the spatial, temporal, and semantic relationships between the manipulated stimuli (Doehrmann and Naumer, 2008; Alais et al., 2010). The same methods cannot be directly applied to study body ownership. For example, it is not possible to introduce a temporal delay between seeing our fist striking the table and feeling the punching sensation in the hand, unless through the use of devices such as cameras and displays.

Given the limitations inherent in the study of the physical body, experimental research has been extensively conducted through exploiting the illusions of body ownership. In these illusions, healthy adults experience non-bodily objects (e.g., artificial limbs) as belonging to their own body, when presented with crossmodal stimuli applied to the hidden real body part and its fake counterpart. Due to the fact that the stimuli have two independent sources (i.e., the real and the fake body part), experimenters have been able to flexibly manipulate their spatial, temporal, and semantic relationships. In this way, body ownership illusions (BOls) therefore offer a powerful experimental tool to examine how the sense of body ownership emerges from multisensory processing operated by the brain.

Our understanding of how the brain builds the sense of body ownership can benefit from identifying the basic principles that govern the induction of body ownership illusions and formulating the latter within a computational framework. Consequently, the aim of this paper is two-fold. First, to review the experimental literature, investigating how the spatial, temporal, and semantic congruencies of the tested crossmodal stimuli contribute to eliciting body ownership illusions. Second, to discuss and propose different theoretical accounts that could possibly cast the experimental findings into a unifying computational context. We start by introducing body ownership illusions and distinguishing these from other classes of body illusions that, together with the former, have provided essential insights on how the own-body percept is built through multisensory and sensorimotor information. Following this, we present the main results of experimental work on body ownership illusions, classifying them on the basis of the crossmodal triggers and semantic information that these have manipulated. This classification is functional to the discussion of different theoretical accounts of body ownership.

\section{Body Illusions to Study OWn-Body Perception}

Body illusions refer to those psychological phenomena in which the perception of one's own body importantly deviates from the configuration of the physical one, e.g., in terms of size, location, or ownership. Since their induction is achieved through multisensory and/or sensorimotor stimulation, body illusions provide essential insights on how the own-body percept is built in real-time, on the basis of the stimuli that are currently available to the brain.

A representative example of body illusions are the body distortions illusions, in which people can 
perceive that the size or the posture of their body part(s) have changed dramatically without necessarily satisfying the anatomical constraints of the human body. A method to induce illusory body distortions relies on kinaesthetic illusions, in which blindfolded subjects experience the illusory movement of a static body part and therefore non-veridical proprioceptive states, when the tendon muscle of a physically constrained joint is mechanically vibrated (Goodwin et al., 1972). Importantly, the illusory motion of the stationary body part can capture other non-movable body parts it is in contact with, yielding the impression that these change in size (Lackner,1988; deVignemont et al.,2005; Ehrsson et al., 2005b). Similar distortion illusions have been shown to occur for numerous other body parts (Lackner, 1988), to correlate with activation of areas in the lateral parietal cortex (Ehrsson et al., 2005b) and to modulate the tactile processing on the body part perceived as distorted (de Vignemont et al., 2005). A well-known example is the Pinocchio illusion (Figure 1A). Alternatively, a distortion illusion similar in phenomenology can be induced through temporal correlations between undistorted proprioceptive information and tactile input, as for example the phantom nose illusion (Ramachandran and Hirstein, 1998) (Figure 1B). Independently of the employed methodology, the body distortion illusions demonstrate that the brain computes the perceived body posture and shape dynamically and in a flexible fashion, without the need to satisfy the anatomical constrains of the human body.

Another remarkable class of body illusions are the experimentally induced out of body experiences, in which people perceive their self to be dislocated from the spatial origin of their visual perspective (Lenggenhager et al., 2007), and/or of looking at their body from a distance (Ehrsson, 2007). One of the first reports of these illusions was attained through the use of a mirror device and goes back to the end of the nineteenth century (Stratton, 1896). In the recent experimental settings participants wear a head mounted display (HMD), which is fed by a camera that records the back of the participants from distance. Thus, participants see their body from the back, i.e., from a third person visual perspective ( $3 \mathrm{PP})$, in contrast with the first person visual perspective (1 PP) that we normally have on our body when looking downward. Through applying tactile stimulation on the participants' back while they see the instrument touching their back in the online video, participants experience illusory drifts in their self-location toward the body seen in the video (e.g., Lenggenhager et al., 2007) (Figure 1C). Illusory changes in self-location were also reported when subjects were tapped on their chest with a stick while another stick was synchronously waived underneath the cameras (e.g., Ehrsson, 2007). Analogous illusory experiences were reported by subjects standing between two mirrors that face each other, while stroking their cheek and watching the several reflections of their bodies (Altschuler and Ramachandran, 2007). Systematic experimental findings from the out of body illusions demonstrate that the perceived location of the self can be detached from that of the physical body, similar to reports from patients undergoing out-of-body experiences of neurological origin (Blanke and Mohr, 2005). This class of illusions provides therefore an important benchmark for studying the role of multisensory integration in the sense of self-location and selfconsciousness (Blanke and Metzinger, 2009; Blanke, 2012). 

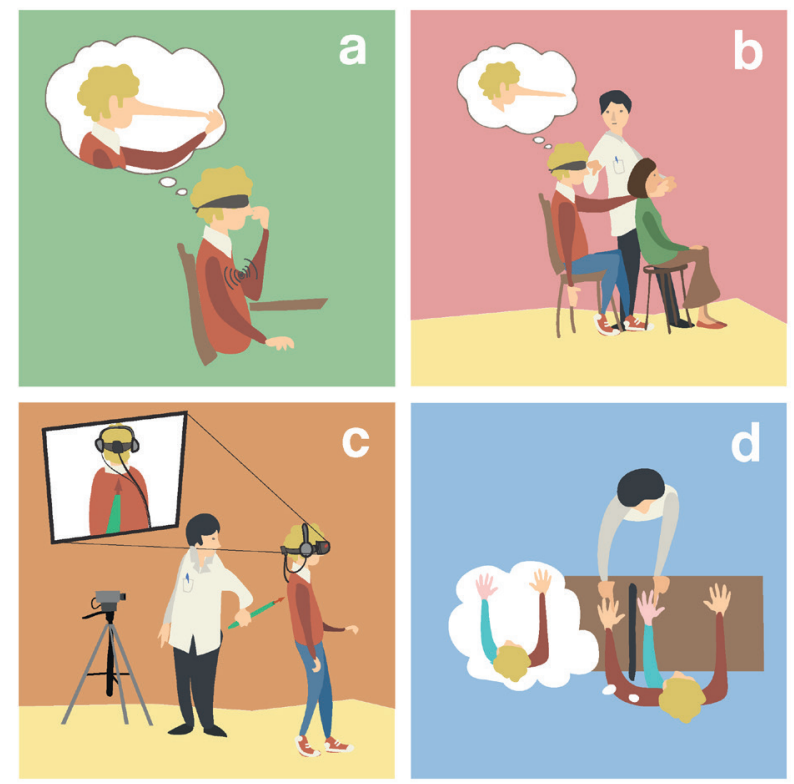

Figure 1. Examples of body illusions. (A) The Pinocchio illusion. A blindfolded participant receives vibration on his biceps while touching the tip of his nose with his fingers. The illusory extension of the arm (Goodwin et al., 1972) generates the illusion that his nose, his fingers or both are elongating (Lackner, 1988). (B) The phantom nose illusion. The experimenter moves the finger of a blindfolded participant to tap the nose of another subject, while simultaneously tapping the nose of the participant. As the participant's movements and his finger contact with the other subject's nose are synchronous with the touch he receives on his nose, the participant experiences the illusion of tapping his very long nose (Ramachandran and Hirstein, 1998). (C) An out of body illusion. The participant sees a video of his back as if he were located behind it. The experimenter touches the back of the participant with a stick while the participant sees it online in the video. As the seen and the felt stimulation is synchronous, the participant perceives illusory drifts in his selflocation toward the seen body (Lenggenhager et al., 2007). (D) The rubber hand illusion. The participant sees a rubber hand placed in front of him, while his real hand is concealed from view. The experimenter strokes both hands at the same time, and after some time the participant perceives the fake hand as if it were his own hand (Botvinick and Cohen, 1998).

\subsection{Body OWNERShip IlLusions}

In distinction to body distortion and out of body illusions, Body ownership illusions refer to the illusory perception of non-bodily objects (e.g., artificial limbs) as being parts of one's own body and as being the source of the associated bodily sensations, such as touch. For example, one can get to experience a mannequin's hand as his/her own hand, and have strong physiological responses when seeing it being attacked with a knife (Ehrsson et al., 2007). 
One of the earliest versions of this class of illusion was reported by Tastevin (1937), who described how people could perceive an artificial finger protruding from a cloth as their own finger, when the latter was hidden from view. Sixty years later, Botvinick and Cohen (1998) provided the first report of the rubber hand illusion ( $\mathrm{RHI}$ ), in which healthy adults experience a rubber hand as if it were their own hand. In this experimental setup, subjects have their hand concealed from view, while they see a rubber hand of the same laterality, placed in a similar posture. The experimenter strokes both rubber and real hands simultaneously, and after some time the majority of participants report perceiving the touch as if coming from the rubber hand, and the latter as being part of their own body (Figure 1D). In addition, when asked to point blindly to the position of their left hand, participants typically give proprioceptive estimations that are shifted toward the rubber hand compared to their estimations before the stimulation (e.g., Botvinick and Cohen, 1998; Tsakiris and Haggard, 2005).

Apart from proprioceptive drifts, the RHI has been shown to decrease the temperature and slow down the processing of tactile input from the real hand (Moseley et al., 2008; Hohwy and Paton, 2010) and to trigger the participants' autonomic responses when seeing the rubber hand under threat (e.g., Armel and Ramachandran, 2003). Interestingly, it has been shown that brain areas associated with anxiety and interoceptive awareness selectively activate when, during the RHI, the fake limb is under threat and at a similar level as when the real hand is threatened (Ehrsson et al., 2007). Moreover, the subjective reports about the intensity of the illusion, typically addressed through questionnaires, have been shown to correlate positively with many of the objective measures mentioned abovee.g., proprioceptive drifts (Longo et al., 2008b), brain activity (Ehrsson et al., 2004; Limanowski et al., 2013), and decrease in temperature of the real counterpart (Moseley et al., 2008).

Beside correlated visuotactile cues, the RHI has been demonstrated also under different multimodal stimuli. For example, the illusion has been induced with correlated visual and motor information, that is when participants are performing movements with their unseen body part while seeing the fake counterpart moving similarly (e.g., Dummer et al., 2009; Kalckert and Ehrsson, 2012, 2014a,b). In addition, just seeing the rubber hand at the same position of the occluded real hand, that is in the mere presence of coincident visual and proprioceptive information, can elicit the illusion of body ownership (Giummarra et al., 2010).

The illusions of body ownership have been also induced toward full humanoid bodies, by using HMDs that permit seeing the fake body in the same spatial location as the real body (Petkova and Ehrsson, 2008; Slater et al., 2010; Petkova et al., 2011b; Maselli and Slater, 2013). These full body illusions were induced through visuotactile (e.g., Petkova and Ehrsson, 2008), visuomotor (e.g., Peck et al., 2013), and visuoproprioceptive information (Maselli and Slater, 2014). Moreover, they were shown to have physiological and proprioceptive correlates analogous to those of the RHI (Petkova and Ehrsson, 2008; Llobera et al., 2013; Maselli and Slater, 2013, 2014).

The induction of these illusions was shown even in the absence of any visual input. In the so-called "somatic RHI," blindfolded participants have their left index finger passively moved by the experimenter to touch a rubber hand, whereas the experimenter synchronously touches their right hand. After some seconds of correlated tactile and proprioceptive information, the participants typically report the illusion of touching their own hand, instead of the rubber one (Ehrsson et al., 2005a). Although 
using a different methodology, the somatic RHI was found to correlate selectively with enhanced activity in the premotor cortex, as in the classic RHI and the full body ownership illusion (Ehrsson et al., 2004, 2005a; Petkova et al., 2011a). This remarkable convergence of results supports the view that body ownership is not determined by the type of sensory triggers employed, but emerges from the synergetic processing of the multimodal information available at a specific time.

Extending the findings from other bodily illusions, body ownership illusions reveal that our brain dynamically computes which are our own body parts on the basis of the available multisensory and sensorimotor information. Nevertheless, although the numerous studies bring along a wealth of insights about the multisensory mechanisms that underlie own-body perception, little emphasis has been devoted to casting this information into a coherent and comprehensive picture. In the following, we review the literature on body ownership illusions with the aim of identifying the multimodal triggers and constraints that govern them. Given the different response variables used by different experimenters, our criterion about the impact of the manipulation of the experimental variable in eliciting the illusion is primarily based on the most common measure, the subjective scores collected through questionnaires. However, we also refer to other objective measures especially in the cases where no questionnaires were administered. Moreover, given the extensive literature, we limit our scope to the body ownership illusions where visual information was available.

\section{Multimodal Triggers and Semantic Constraints in BODY OWNERSHIP ILLUSIONS}

The elicitation of body ownership illusions (BOls) has been shown under different experimental setups that provide different crossmodal stimuli (Figure 2). In this section, we present several experimental studies on BOls by classifying them in terms of the main crossmodal stimuli provided (i.e., visual and tactile, visual and proprioceptive, and visual and motor), or of the semantic feature of the fake body that these have manipulated. With the specific aim to highlight the principles that permit the induction of BOls, we investigate the importance of spatial and temporal correspondence for each pair of crossmodal stimuli, as well as the role of semantic information that the view of the non-bodily objects brings along. To provide a link between the illusions of body ownership and our normal sense of body ownership, for each of considered the components we present a brief overview of its known role in our own-body perception. 

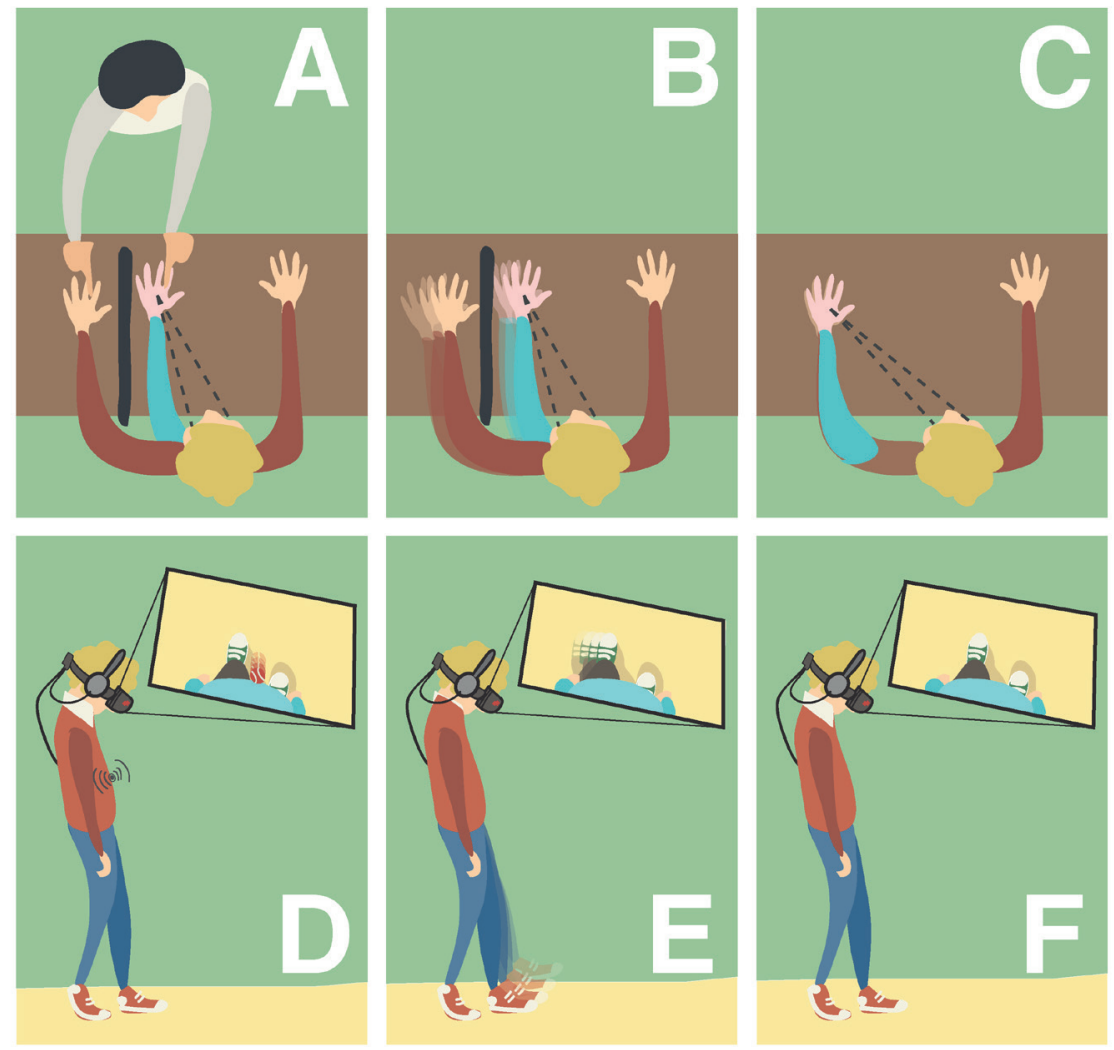

Figure 2. Different induction methods of Body Ownership Illusions (BOIs). (A,D) Visuotactile triggers: the participant is watching the fake hand/body placed in a plausible posture and being touched, while receiving synchronous tactile stimulation in the real counterpart that remains out of view. $(B, E)$ Visuomotor triggers: the participant is performing movements with his real hand/body that remains out of view, while watching the fake counterpart moving synchronously. (C,F) Visuoproprioceptive triggers: the participant is watching the fake hand/body placed in an overlapping position with the real counterpart that remains out of view.

\subsection{Visuotactile Triggers for Body OWNership Illusions}

\subsubsection{Visuotactile Integration in OWn-Body Perception}

The experience of our own body is importantly shaped by visual and somatosensory signals. One of the main contributions of visuotactile integration concerns the information about contact events between our body and the surrounding environment. For example, when a fly lies on our hand, both 
vision and touch inform the brain about the time and the location of the contact. But even before the physical contact occurs, vision can provide anticipatory information about where and when the fly is probably going to land, which allows us to take actions accordingly.

Beside its established role in determining the shape and texture of objects we explore with our hands (Ernst and Banks, 2002; Lacey et al., 2010; van Beers et al., 2011; Helbig et al., 2012), visuotactile integration is considered to be critical in perceiving our body and the space nearby. This was first suggested by the discovery of visuotactile neurons in the brain of monkeys: the visual receptive fields of these cells extends outwards from the mapped body part into the external space and shifts along with body movement (Rizzolatti et al., 1981; Graziano and Gross, 1993; Duhamel et al., 1998). Because of these properties, these bimodal neurons were proposed to build an enlarged and flexible representation of the space surrounding the body - the peripersonal space-that mainly serves to guide our movements (Graziano and Gross, 1994; Fogassi et al., 1996; Graziano and Cooke, 2006).

Homologous visuotactile populations have been identified in the human brain with analogous properties and functionality (Lloyd et al., 2003; Makin et al., 2007; Gentile et al., 2011; Sereno and Huang, 2014). In addition, the importance of visuotactile integration in the spatial representation of the body, has been largely supported by behavioral studies (Spence et al., 2004).

Altogether, experimental evidence from monkey neurophysiology to human behavior indicates the fundamental role of visuotactile populations in flexibly defining the dynamics of our own body spatial boundaries.

\subsubsection{Methods and Apparatus for Visuotactile Stimulation in BOls}

Turning to $\mathrm{BO}$ s, the first report of the $\mathrm{RHI}$ was induced through applying tactile stimuli on the occluded real hand and the visible fake counterpart (Botvinick and Cohen, 1998). The procedure was quickly established as a standard protocol and extended to full BOls as well (Figures 2A,D).

With respect to the employed methods, seen and felt touch have been typically delivered manually by the experimenter (e.g., Botvinick and Cohen, 1998; Armel and Ramachandran, 2003; Petkova and Ehrsson, 2008). Alternative setups used cameras, screens or virtual reality methods. By these technological means, some experimenters manually stimulated only the real body (or body part), whilst the participants watched a video stream of the real body part being touched (e.g., Tsakiris et al., 2006) or a virtual object touching an artificial body (or body part) seen in stereo mode (e.g., Slater et al., 2008). Alternatively, computer-controled administration of touch was implemented by programming robot arms or stepper motors that touched both the real and the fake body (or body parts) (Tsakiris et al., 2008; Rohde et al., 2011), or by attaching mechanical vibrators on the real body and programming them to fire when the participants watched the virtual body being touched through the HMD (Pabon et al., 2010; Evans and Blanke, 2013; Maselli and Slater, 2013).

\subsubsection{Spatial and Temporal Principles of Visuotactile Integration as Trigger of BOls}

Systematic experimental evidence has shown that the RHI is induced when the real hand and the fake counterpart are touched at the same time and at homologous regions in hand-centered 
coordinates. For example, when spatiotemporal mismatches were introduced between the seen and felt touch, the RHI was significantly inhibited (Armel and Ramachandran, 2003; Ehrsson et al., 2004; Slater et al., 2008). Similar results were found for full BOls toward plastic mannequins seen from a 1 PP; participants perceived the mannequin's body as their own body when the real and the fake abdomen part were touched synchronously, but not when asynchronously (Petkova and Ehrsson, 2008). Nevertheless, other studies have shown that when the fake body (or body part) is realistic and has overlaps in space with the real body counterpart, positive scores of ownership can be reported even in presence of asynchronous visuotactile stimulation (Longo et al., 2008a; Maselli and Slater, 2013).

A systematic study on the importance of temporal alignment revealed that, when delays smaller than 300 ms between the stimulations were introduced, participants perceived the touch on their hand as if caused by the stimulation applied to the rubber hand, while for larger delays these illusory feelings were significantly attenuated (Shimada et al., 2009, 2014). In addition to the temporal coupling, spatial congruence was also found to be essential for the induction of the illusion. Stroking the rubber and the real hands in temporal synchrony but at different locations (e.g., index vs. little fingers, palm vs. forearm, or index vs. middle finger) abolished the illusion (Kammers et al., 2009; Limanowski et al., 2013; Riemer et al., 2014), demonstrating that temporal matching alone is not sufficient for the RHI. Similar were the findings on proprioceptive drifts when a spatial mismatch was introduced between the seen and the felt synchronous strokes (Costantini and Haggard, 2007).

\subsubsection{SUMMARY}

Overall, the experimental results suggest that the induction of BOls depends critically on the spatiotemporal congruence between the seen and the felt stimulation, which is a sufficient condition to induce the illusions. However, the fact that BOls could occur in presence of visuotactile asynchronies when the fake body is seen superimposed onto its real counterpart, indicates that visuotactile correlations are not a necessary prerequisite for the illusion under such configuration.

\subsection{VisUomotor Triggers for BOdy OWNERSHIP ILLUSIONS}

\subsubsection{Visuomotor Integration in OWn-Body Perception}

Our own-body perception is highly determined by our movements since these provide information that facilitates self-other distinction. For example, when a pianist plays the piano in a duet, she usesamongst others - her motor information together with visual, tactile and proprioceptive feedback to recognize which of the two right hands she sees is her own.

The experience of moving and acting in space involves an extremely rich content of body related information that goes far beyond the multisensory integration of two or more sensory modalities. This can be better understood considering the distinction between active vs. passive movements. In a passive movement there is no motor intention: an externally generated force displaces our body (body part) and consequently, a number of sensory information, including vision and proprioception, are updated (Burke et al., 1988). In contrast, when we perform an active movement, our brain 
generates and implements a motor plan that relies on internal simulations of our motor system (Wolpert et al., 1995): the motor plan is executed, monitored and corrected by comparing the efference copy with the generated sensory feedback (Miall and Wolpert, 1996; Todorov and Jordan, 2002). The implication of internal body simulations renders the processing of visuomotor cues during active movement much richer in body-related information content. Indeed, this is supported by experimental evidence. For example, it was shown that participants are better at discriminating synchronous vs. asynchronous visuomotor cues when performing active movements compared to passive (Shimada et al., 2010).

It is also relevant that visual monitoring and recognition of one's own actions has been proposed to contribute critically to the sense of body ownership (Jeannerod, 2003), and to play a major role in self-other discrimination (Jeannerod, 2004). Developmental studies have shown that, at 4to 5-months of age, infants are already able to distinguish their limbs from those of other babies on the basis of synchronous visuomotor correlations: they can distinguish an online video stream of their own moving limbs from similar videos of other babies, from delayed videos of their own moving limbs, (Bahrick and Watson, 1985; Schmuckler, 1996), as well as from online videos in which their body spatial configuration is seen modified (Rochat and Morgan, 1995; Schmuckler, 1996).

A set of experimental studies has shown how healthy adults rely on visuomotor stimuli to discriminate whether a seen moving hand is their own. For example, in the experiment of Daprati and colleagues, participants were asked to perform a movement while looking at a screen in which their filmed hand or the experimenter's was displayed in the same position of their occluded hand. When asked to indicate whether the seen hand was their own hand, participants' performance showed a high rate of erroneous self-attribution when seeing the experimenter's hand doing the same movement (Daprati et al., 1997). In a similar setup, participants saw a virtual hand moving as their own occluded hand, under different spatial orientations and temporal delays. When asked to decide whether the displayed movement corresponded to their own movement, subjects made significantly more self-recognition errors for temporal delays smaller than $150 \mathrm{~ms}$ and for angular deviations smaller than $15^{\circ}$ (Franck et al., 2001). A variation of the same setup was used to investigate how action and visual cues about the body spatial configuration are used in bodyrecognition processes (van den Bos and Jeannerod, 2002). Participants were presented with an online video of their own hand and the hand of the experimenter performing either the same, different or no movement at all, while displayed simultaneously in different orientations. The results showed that when the hands performed different movements, participants were almost always able to recognize their own hand. In contrast, when the visuomotor information was ambiguous (same movements) participants were less accurate and their responses were affected by the seen spatial configuration of the hands.

Overall, it becomes apparent that humans exploit the tight coupling of visual and motor signals not only for optimizing their movement performance, but also for attributing their body parts and body movements to themselves.

\subsubsection{Methods and Apparatus for Visuomotor Stimulation in BOls}

The induction of BOls has been demonstrated in presence of visuomotor stimulation instead of 
visuotactile. In those experimental setups, the participants perform either active or passive movements while seeing the artificial body (or body part) moving (Figures 2B,E).

The animation of the fake body has been typically achieved through mechanical devices that permitted linking the real and the fake body parts, such as wooden rods (Kalckert and Ehrsson, 2012, 2014a), pulleys and strings (Azañón and Soto-Faraco, 2007), couplings (Walsh et al., 2011), pneumatically driven plungers (Riemer et al.,2013,2014), and braces (Dummer et al.,2009).Alternative experimental setups used cameras to record the participants' moving hand and project it to a surface or a screen (Tsakiris et al., 2006, 2010b). The intrinsic delays of the apparatus are typically of the order of 70100 ms (Tsakiris et al., 2006; Riemer et al., 2013, 2014), and thus below the threshold of 150 ms for detecting visuomotor delays (Franck et al., 2001; Shimada et al., 2010). Other experimenters, using virtual reality techniques, captured the participants' movements-through inertial systems and/or infrared cameras - and animated the virtual limbs or bodies accordingly (Sanchez-Vives et al., 2010; Yuan and Steed, 2010; Normand et al., 2011; Kilteni et al., 2012, 2013).

\subsubsection{Spatial and Temporal Principles of Visuomotor Integration as Trigger in BOls}

Several studies have demonstrated that BOls are induced when both real and fake bodies move homologous body parts at the same time. This has been shown, for example, with fingers (Tsakiris et al., 2006, 2010b; Sanchez-Vives et al., 2010;Walsh et al., 2011; Kalckert and Ehrsson, 2012, 2014a,b; Riemer et al., 2013), hands (Dummer et al., 2009), arms (Yuan and Steed, 2010; Normand et al., 2011; Kilteni et al., 2012; Llobera et al., 2013), upper body (Kilteni et al., 2013), legs (Kokkinara and Slater, 2014), or full bodies (Banakou et al., 2013; Peck et al., 2013). In contrast, when introducing temporal delays of the order of 500 ms or more (Kalckert and Ehrsson, 2012, 2014a; Riemer et al., 2013, 2014), or when manipulating the seen movements independently from the real movement (Dummer et al., 2009; Sanchez-Vives et al., 2010;Walsh et al., 2011; Banakou et al., 2013), the corresponding illusions were inhibited. In addition to the temporal coupling, moving homologous real and fake body parts was also found to be essential for the induction of the illusion; for example, moving the index finger while seeing the artificial middle finger moving synchronously did not elicit the ownership illusion (Riemer et al., 2014).

\subsubsection{SUMMARY}

Similarly to visuotactile stimuli, the experimental results suggest that the induction of BOls depends critically on the spatiotemporal congruence between the seen and the felt movements.

\section{3. Visuoproprioceptive Modulations ANd Triggers for Body OWNERSHIP ILLUSIONS}

\subsubsection{Visuoproprioceptive Integration in OWn-Body Perception}

Our own-body perception is greatly influenced by visual and proprioceptive information. The main contribution of visuoproprioceptive integration relies on defining where our body is in space. Both modalities inform the brain about where a specific body part is located at a given point in time. For example, we can estimate where our hand is with our eyes closed, and our judgement can further 
be confirmed by looking at our own hand.

Experimental evidence has shown that when vision and proprioception provide conflicting information, the perceived position of our body parts can significantly deviate from the veridical one. For example, when the hand is seen in a non-veridical location through mirrors or prisms, subjects perceive their hand to be in one single location, somewhere between the seen and the felt position (Hay et al., 1965; Pick et al., 1969; Warren, 1980) and closer to the visual one (van Beers et al., 1999). This visual capture of proprioception has been shown to influence the performance of reaching movements (Rossetti et al., 1995; Sober and Sabes, 2003; Holmes et al., 2004) and to play a crucial role in monitoring online movement execution (Lajoie et al., 1992; Sober and Sabes, 2003, 2005; Bagesteiro et al., 2006; Boulinguez and Rouhana, 2008). Interestingly, a recent study with 5-7 years old children has shown that the visual capture effects increase with age, suggesting a progressive maturation of visuoproprioceptive integration (Bremner et al., 2013).

Analogously to the visual capture of proprioception, it has been shown that in absence of direct vision of the body, proprioception can distort the perception of visual cues associated to the body spatial configuration. For example, participants sitting in a dark room while holding a luminous target with their static hand, report seeing the (static) luminous target moving in space when experiencing the kinaesthetic illusion of moving their (static) arm (Lackner and Levine, 1978). Similarly, moving the hand while seeing its afterimage (Gregory et al., 1959) was shown to distort the hand image or even to fade it out, despite that this should be perceptually static on the sole basis of visual input (Davies, 1973).

Most studies on visuoproprioceptive populations have been carried out in the context of reaching actions and motor control in both monkeys (e.g., Battaglia-Mayer et al., 2000) and humans (e.g., Filimon et al., 2009). Nevertheless, neural populations have been found in the monkey brain, which integrate static visual and proprioceptive cues to encode the position of the arm (Graziano et al., 2000). To our knowledge, no analogous studies have been performed with humans.

Overall, experimental evidence suggests that visual and proprioceptive information significantly contribute to the perception of our own body and more particularly, in determining its perceived spatial configuration.

\subsubsection{Methods and Apparatus for Visuoproprioceptive Manipulations in BOls}

BOls, including their full body versions, have been tested against differences between the seen spatial configuration of the fake body (or body part) and the one of the real body (or body part) that is experienced through proprioception.

In its original version (Botvinick and Cohen, 1998), the RHI was induced despite the spatial mismatch between the positions of the real and the rubber hand. The same spatial arrangement, with the rubber hand closer to the participants' body midline (Figure 2A), was quickly established as a standard protocol for subsequent studies (e.g., Armel and Ramachandran, 2003; Tsakiris and Haggard, 2005; Haans et al., 2008; Moseley et al., 2008; Schütz-Bosbach et al., 2009). In other studies, the rubber hand was instead placed above the occluded real one (e.g., Pavani et al., 2000; 
Ehrsson et al., 2004;Azañón and Soto-Faraco, 2007; Haggard and Jundi, 2009).

The above-mentioned studies used a physical object (i.e., the rubber hand) and their setups therefore entail intrinsic limitations in the possible manipulations of visuoproprioceptive stimuli; it is in fact never possible to have the two hands overlapping in space without using devices. Different techniques including mirrors (e.g., Longo et al., 2008a; Zopf et al., 2010), cameras (e.g.,Petkova and Ehrsson, 2008; Hohwy and Paton, 2010) and virtual reality techniques (e.g., Perez-Marcos et al., 2012), permitted experimenters to overcome this limitation by projecting the fake body (body part) in complete overlap with its real counterparts.

Manipulations of the position and orientation of the fake body (body parts) relatively to the real body, allowed experimenters to investigate the extent to which visuoproprioceptive discrepancies affect the induction and strength of BOls. Furthermore, it was possible to test whether close correspondence in the seen and felt spatial configurations (i.e., apparent spatial coincidence) can trigger BOls without further crossmodal stimulation (e.g., visuotactile) (Figures 2C,F).

\subsubsection{Visuoproprioceptive Modulations of BOIs}

\section{The case of visuotactile triggers}

As long as the fake hand was placed close to the body midline and thus within the reaching space, the $\mathrm{RHI}$ was induced in presence of spatiotemporally congruent visuotactile stimulation, for several tested mismatches between the positions of the real and fake hands in either the horizontal or the vertical plane; e.g., of 10-20 cm (e.g., Zopf et al., 2010; Rohde et al., 2011; Kalckert and Ehrsson, 2014b), 20-40 cm (e.g., ljsselsteijn et al., 2006; Haans et al., 2008; Kalckert and Ehrsson, 2014b), or above $40 \mathrm{~cm}$ (e.g., Ehrsson et al., 2004; Zopf et al., 2010).

With respect to the horizontal distance, no significant differences were found in the strength of the RHI under small (i.e., $15 \mathrm{~cm}$ ) or large (i.e., $45 \mathrm{~cm}$ ) distances between the hands (Zopf et al., 2010), suggesting that the between hands' distance is not a crucial factor. Different results have been found when the rubber hand was placed farer away from the participant's body midline than the real one. In this case, increasing the horizontal distance between the two hands can eventually prevent the $\mathrm{RHI}$ even when the rubber hand still lies within the reachable space (Preston, 2013). This different effect of visuoproprioceptive mismatch on the elicitation of the RHI can probably be explained by our higher expectation of seeing our hand closer to the midline than away from it. The between hands horizontal distance was instead found to significantly affect subjective scores for conditions of asynchronous visuotactile stroking (Zopf et al., 2010): ownership scores were significantly higher when the two hands were as close as $15 \mathrm{~cm}$ with respect to the case in which the distance was larger $(45 \mathrm{~cm})$. In her study Preston (2013) did not find differences in questionnaire scores, which were equally low, independently on the between hands distance. The different results may be due to the different delays adopted in the asynchronous condition (about $300 \mathrm{~ms}$ and $1 \mathrm{~s}$, respectively): in fact the delay used by Zopf and colleagues was reported to mark the upper limit for temporal discrepancies, above which the RHI is significantly weakened (Shimada et al., 2009, 2014).

With respect to the vertical plane, no significant differences in ownership scores were detected 
between placing the real and the fake hands at a vertical distance of 12 or $27.5 \mathrm{~cm}$, though keeping both at the same horizontal position close to the participants' midline. However, increasing the vertical distance between the hands to $43 \mathrm{~cm}$ was found to significantly attenuate the RHI scores (Kalckert and Ehrsson, 2014b).

While in all the above-mentioned studies the real and fake hands differed in position but were mostly aligned in terms of orientation, other experiments have shown that BOls can be induced in presence of an additional mismatch in orientation, provided that the fake body (body part) was seen in an anatomically plausible posture. For example, rotating the fake left hand by $44^{\circ}$ clockwise did not prevent participants from experiencing the physical touch as if coming from the position of the rubber one, and further led to a recalibration of their perceived elbow joint angle (Butz et al., 2014). BOls were experienced also when the seen fake hand, rotated away from the real one, crossed the body midline (Brozzoli et al., 2012; Perez-Marcos et al., 2012); but see (Costantini and Haggard, 2007; Cadieux et al., 2011) for effects in proprioceptive drifts. In addition, the RHI was induced toward a rubber hand placed palm-up when the fake palm was stroked synchronously with the palm of the real that was placed palm-down (lonta et al., 2013). Moreover, by exploring different orientation mismatches, a recent study reported that tactile sensations were perceived to arise from the rubber mainly for those rotations that are easy to mimic with the real. Interestingly, the illusion onsets were found to be shorter when there were no orientation mismatches, without though any significant differences in the subjective reports (Ide, 2013). Instead, when discrepancies in both position and orientation were introduced by moving the rubber hand away from the participants' midline and rotating it, illusory tactile sensations were reported to gradually decrease in intensity with effects also in illusion onsets (Lloyd, 2007).

Consistent with these are the findings from full BOls. These have been typically induced toward artificial bodies that appeared as if spatially coincident with the real ones, seen therefore from a $1 \mathrm{PP}$, and under spatiotemporally congruent visuotactile stimulation (Petkova and Ehrsson, 2008; Slater et al., 2010; Petkova et al., 2011b; Maselli and Slater, 2013). With respect to position, a recent study showed that a full BOI could be experienced toward a virtual body seen from a laterally shifted visual perspective (by about $25 \mathrm{~cm}$ ), i.e., with the virtual body overlapping only partially with the real one, upon congruent visuotactile stimulation. When compared to a condition when the fake body was in complete overlap with the real one, no significant differences in ownership were detected (Maselli and Slater, 2014).Analogously for orientation, the illusion was induced when the mannequin's body was seen from a 1 PP but tilted upwards by $30^{\circ}$ approximately (Petkova et al., 2011a).

\section{The case of visuomotor triggers}

Beside the information about the movement timings and the involved body parts, visuomotor stimuli provide information on how the relative position and orientation of the real and fake bodies change in time. Some of the studies that employed visuomotor stimulation of artificial body parts followed the RHI protocol and presented the artificial limb close to the midline and lateral to the real one, e.g., at a distance of 15-20 cm (e.g., Sanchez-Vives et al., 2010; Riemer et al., 2013, 2014) while others placed the fake limb above the real one at a vertical distance of about $12 \mathrm{~cm}$ (e.g., Walsh et al., 2011; Kalckert and Ehrsson, 2012, 2014a,b). With respect to the horizontal distance, 
no significant differences were found in the subjective reports of body ownership when the fake and the real limb moved synchronously while keeping a distance of about $10 \mathrm{~cm}$, compared to when these were spatially coincident (Yuan and Steed, 2010). Nevertheless, larger distances were not tested. With respect to the vertical distance, a recent study revealed that small distances (i.e., 12 $\mathrm{cm}$ ) permitted the induction of a robust RHI through congruent visuomotor stimulation while larger distances (i.e., 27.5 or $43 \mathrm{~cm}$ ) significantly attenuated the subjective scores (Kalckert and Ehrsson, 2014b).

\subsection{4 . VISUOPROPRIOCEPTIVE INTEGRATION AS TRIGgER FOR BOIS}

The fact that full BOls were found to occur when having a static view of a highly realistic spatially coincident virtual body and under asynchronous visuotactile stimulation, suggests that congruent visuoproprioceptive cues alone could be sufficient to induce the illusion and further to sustain it under visuotactile discrepancies (Maselli and Slater, 2013). In partial agreement are findings from the RHI where participants seeing the rubber hand through a mirror, as if spatially coincident with their real one, did not disagree with statements of ownership when the visuotactile stimulation was asynchronous (Longo et al., 2008a).

In contrast to the large number of studies using visuotactile triggers, there have been fewer that explored whether spatial congruency of visuoproprioceptive cues can be by itself sufficient for the induction of BOls. These studies, in which BOls were assessed in "vision only" conditions, suggest that when there is neither visuotactile nor visuomotor stimulation the relative position and alignment of the real and fake body (body part) matters. When seeing through a mirror a rubber hand in the same position and orientation as the real one, participants gave positive scores for the illusion of ownership (Longo et al., 2008a; Giummarra et al., 2010). Nevertheless, upon direct comparison, additional synchronous visuotactile stroking elicited a significantly stronger illusion (Longo et al., 2008a). In contrast, when the fake and real hands were not spatially coincident, the mere vision of the rubber hand was not sufficient for inducing the illusion (as from participants' self-reports) (Rohde et al., 2011). In line with this overall evidence, it was shown that the visual exposure to a realistic spatially coincident virtual body by itself, can induce a full BOI (as reported in post experiment debriefing) (Maselli and Slater, 2014).

\subsubsection{SUMmarY}

These findings reveal that spatial congruency of visuoproprioceptive cues (i.e., spatial coincidence) is not necessary for the illusion of body ownership to emerge, provided that the fake body (body part) is seen in an anatomical plausible configuration and in presence of congruent visuotactile or visuomotor stimulation. However, different degrees of visuoproprioceptive spatial mismatch significantly modulate both the intensity and the time onset of the illusion.

On the other hand, spatial coincidence may be a sufficient condition for eliciting BOls. Although there is consensus in the literature that congruent visuotactile and visuomotor stimulation is necessary for inducing BOls, this may specifically apply to conditions that include visuoproprioceptive mismatches. 


\subsection{Semantic Constraints in Body OWnership Illusions}

\subsubsection{Semantic Constraints in OWn-Body Perception}

Beside the continuously updated sensory and motor information, our own-body perception largely relies upon higher-order, cognitive processes. Our semantic memories and knowledge contribute in shaping an abstract body model that contains information about the general and not self-specific visual, postural and structural properties of the human body. For example, we know how the human body is structured (e.g., the body has two hands), as well as how many degrees of freedom it has when it moves.

Given their non-self-specific nature, these cognitive processes depend, to an important extent, on neural mechanisms and brain areas that evolved for the visual perception of others' human bodies. Apart from brain areas specifically devoted to face perception (Haxby et al., 2000), several specialized regions for visual processing of bodies have been found in humans and non-human primates. This applies to hands, bodies (with and without heads), and to anatomically plausible body postures and motion (Peelen and Downing, 2007). Research in infants has shown that these selective areas are already functional at few days/months (Gliga and Dehaene-Lambertz, 2005; Hirai and Hiraki, 2005; Reid et al., 2006; Simion et al., 2008), suggesting that we are born equipped with structures for the visual encoding of body parts and human body kinematics.

Moving the focus away from own-body perception for a moment, semantic information has been proposed to be an important feature of multisensory integration. When considering two or more crossmodal stimuli, semantic congruence refers to their "close correspondence of content" (Doehrmann and Naumer, 2008). For example, a visual stimulus showing a dog is semantically congruent with a barking but not with a meowing sound (Alais et al., 2010). In this case, the semantic congruence of the audiovisual stimuli speaks in favor of a common underlying cause: a dog. Several studies have shown that the integration of crossmodal stimuli is enhanced when these are semantically congruent (Doehrmann and Naumer, 2008).

The relevance of semantic congruence in the context of own-body perception has been demonstrated in non-human primates with the seminal work of Graziano et al. (2000). The authors showed that the activity of visuoproprioceptive neurons in the monkey brain is importantly modulated by the semantic content of the visual stimulus. Single cell activity was recorded while manipulating the position of the occluded monkey's arm, and that of a seen object. The object was either a replica of the arm or another object, such as a white paper or an apple's slice. The activity of the neurons was significantly modulated by the position of the arm replica, if the latter was in an anatomically plausible configuration. In contrast, changing the position of the white paper, or of the apple slice, had no effect. Interestingly, if the arm replica was seen in non-anatomically plausible configurations, i.e., with opposite handedness or rotated by $180^{\circ}$ so that the fake fingers were pointing to the chest, the observed activity was no longer affected by the fake arm's position (Graziano et al., 2000).

Altogether, experimental evidence suggests that our brain is equipped with highly specialized structures for the visual processing of body parts and, in order to be effectively integrated with 
the somatosensory signals, visual cues should not only resemble body parts but further satisfy body semantic constraints in terms of anatomical plausibility of posture and structure.

\subsubsection{Semantic Constraints in BOls}

In the particular context of BOls, the level of semantic congruence refers to which extent the noncorporeal object resembles a not self-specific human body (body part) in terms of shape, anatomy and structure. We first distinguish between objects with human body shape or not (Figures 3A,B). If the objects are body-shaped, their semantic information can be further characterized by their texture, the anatomical plausibility of their spatial configuration and the anatomical plausibility of their internal structure (Figures 3C-E).
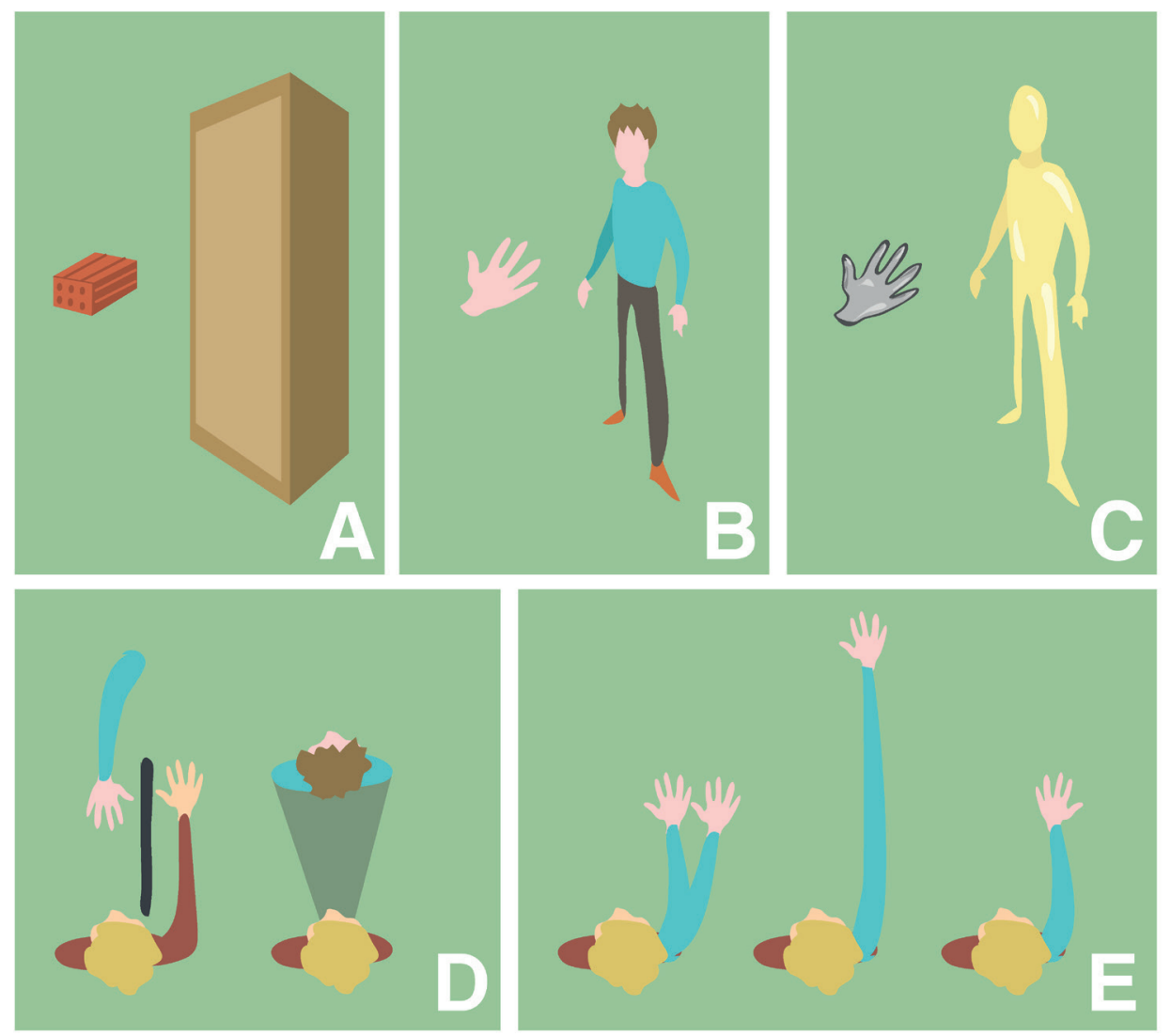

Figure 3. Examples of objects with different semantic information. (A) Objects with non-human body shape. (B) Objects with human body shape. (C) Objects with non-human skin texture (D) Objects (blue) in anatomically implausible spatial configurations with respect to the participant's body (red). (E) Objects (blue) with anatomically implausible structure with respect to the human body. 


\subsubsection{The Role of Shape}

Converging experimental evidence suggests that $\mathrm{BO}$ s are shape-sensitive. For example, when a checkerboard (Zopf et al., 2010) was used instead of a rubber hand, the reports of the illusion were weaker if not entirely absent. Analogous inhibition in subjective reports was found for non-body shaped objects with hand-like skin texture (Haans et al., 2008) or size (Hohwy and Paton, 2010, experiment 3). Similar inhibitory effects were also detected for proprioceptive drifts when using a stick instead of a rubber hand (Tsakiris and Haggard, 2005).Analogously, full BOls were suppressed when participants had a 1 PP view of a rectangular body-sized object instead of a 1 PP view of a mannequin (Petkova and Ehrsson, 2008). Furthermore, even smaller violations of shape were shown to have an effect; in the study of Tsakiris et al. (2010a) the RHI was reported only for the rubber hand and not for other objects, including a wooden block with sculpted wrist and fingers.

The results reported in the study ofArmel and Ramachandran (2003) are somewhat in disagreement with these findings: stroking a table surface synchronously with the participants' hidden hand induced weak ownership sensations toward the table, though significantly higher in intensity compared to the control condition in which the real hand was visible. Despite this, subjective scores for the "table ownership" were significantly lower with respect to when a rubber hand was used-a condition that always preceded the table one. Motivated by this point, the study of Hohwy and Paton (2010) showed that while the mere synchronous visuotactile stimulation of a piece of hand-sized cardboard was not sufficient to induce the illusion (experiment 3), prior induction of the illusion (by using a rubber hand) could instead allow the illusion to be sustained and projected on the cardboard when the rubber hand was instantaneously replaced by it (experiment 2).

These results suggest that whereas BOls are critically sensitive to shape, a prior exposure to the illusion can modulate this sensitivity by overriding the contribution of prior knowledge about the shape of human bodies to the illusion itself.

\subsubsection{The Role of Texture Realism}

Semantic information related to the realism of the fake body (body part) has been investigated through modifying its seen material texture. The realism of the seen texture is associated with the degree of the perceived biological plausibility of the seen object as a candidate for a human body part. Texture realism has been shown not to be crucial for BOls; yet, modulations in the intensity of the illusion have been found when manipulating the texture. For example, the RHI has been elicited toward a fake hand wearing a latex glove, though at a significantly lesser intensity compared to when the realistically textured rubber hand was used (Haans et al., 2008). Similarly, a mechanical hand-shaped object made of wires, permitted the induction of the RHI through congruent visuotactile stimulation, albeit to a significantly lesser extent compared to when a realistic replica of a human hand was used (Bertamini and O'Sullivan, 2014). Turning to full BOls, texture realism was proposed to have a modulatory effect when proving visuotactile stimulation (Maselli and Slater, 2013): whilst congruent visuotactile triggers were found to be necessary in eliciting the full $\mathrm{BO}$ toward a spatially coincident plastic mannequin (Petkova and Ehrsson, 2008; Petkova et al., 2011b), using a realistically textured virtual body produced negligible differences between congruent and incongruent visuotactile stimulations (Maselli and Slater, 2013). Nevertheless, congruent visuomotor stimulation with a spatially coincident virtual body having an unnatural purple skin color induced 
the illusion, without significant differences in strength compared to when a realistic skin texture was used (Peck et al., 2013). This last result suggests that visuomotor correlations may tend to saturate the illusion, so that modulations by texture become negligible.

\subsubsection{The Role of Anatomical Plausibility of Spatial Configuration and of Visual Perspective}

The anatomical plausibility of the spatial configuration of the fake body was shown to play a critical role in the induction of BOls. For example, rotating the rubber hand by $180^{\circ}$ (with the rubber fingers pointing toward the participant) prevented the RHI, despite congruent visuotactile (Ehrsson et al., 2004; Holle et al., 2011) or visuomotor (Kalckert and Ehrsson, 2012) stimulation. Analogously, a $90^{\circ}$ rotation of a left rubber hand (with the rubber fingers pointing to the left) was shown to reduce the difference in proprioceptive drifts between synchronous and asynchronous visuotactile stimulation (Tsakiris and Haggard, 2005) and to delay the onset of the RHI (Aimola Davies et al., 2013). A systematic study in which different rotations were applied on the rubber hand, including both anatomically plausible and implausible configurations, has shown that illusory experiences were elicited mainly for anatomically plausible configurations (Ide, 2013).

Apart from violating constraints of human joints articulation, the plausibility of the body spatial configuration can be broken also by manipulating the visual perspective on the fake body or body part. For example, seeing directly one's own body from a distance is anatomically impossible. Similar to findings from the $\mathrm{RHI}$, having a 3 PP over a distant virtual body or a mannequin was found to inhibit the illusion, despite congruent visuotactile stimulation between the real and the fake body. Upon direct comparison of 1 PP and 3 PP conditions, this inhibition was consistently found for different distances at which the fake body was seen: $100 \mathrm{~cm}$ (Slater et al., 2010), $80 \mathrm{~cm}$ (Maselli and Slater, 2013), $75 \mathrm{~cm}$ (Petkova et al., 2011b), and $40 \mathrm{~cm}$ (Maselli and Slater, 2014). In contrast, when the visual perspective was slightly shifted from the eyes of the virtual body, but still consistent with an anatomically plausible view of the own body, the full BOI was induced (Maselli and Slater, 2014). These results are consistent with evidence from RHI experiments that have shown an inhibition of the illusion for rubber hands placed beyond the reaching space. These, as well as other configurations, e.g., the fake hand rotated $180^{\circ}$, could be regarded as cases of having a 3 PP over the fake limb. Interestingly, Bertamini and colleagues induced a vivid RHI providing exclusively a 3 PP view of the rubber hand through a mirror (Bertamini et al., 2011). The apparent disagreement between this result and the findings mentioned above could be explained by the fact that the 3 PP view was provided through a mirror, and considering that we automatically attribute visual information from the mirror to the physical location of the reflected objects. Indeed, the critical role of the mirror in mediating the visual perspective was confirmed within the same study: the RHI was abolished when providing exactly the same visual information about the rubber hand and visuotactile stimulation, but without an intervening mirror.

\subsubsection{The Role of Anatomical Plausibility of Structure}

The impact of anatomical plausibility of the structure of the seen object has been shown to vary importantly depending on the specific manipulation. For example, full BOls have been shown toward artificial bodies of a significantly different scale from the real ones (van der Hoort et al., 2011; Banakou et al., 2013). With respect to the volume of the artificial body (body part), several studies 
have successfully elicited BOls toward two dimensional video projections of either the real or the rubber hand (ljsselsteijn et al., 2006; Tsakiris et al., 2006; Shimada et al., 2009) or toward a normal-sized hand of non-biological texture and of reduced volume (Bertamini and O'Sullivan, 2014), although the illusion was found to be stronger when the fake body part was seen with normal volumetric characteristics (ljsselsteijn et al., 2006). Concerning body connectivity, subjective scores of ownership toward a virtual hand were significantly stronger when this was seen to be connected to the virtual torso rather than disconnected (Perez-Marcos et al., 2012).

With respect to body proportions, several studies that manipulated the seen hands to be either smaller or larger than the average population size, revealed various effects, including weight and size perception (e.g., Haggard and Jundi, 2009; Bruno and Bertamini, 2010; Linkenauger et al., 2013), distance perception (e.g., Linkenauger et al., in press), and movement kinematics (e.g., Bernardi et al., 2013). When studying the particular effect of hand size on eliciting the RHI, high illusion scores without significant differences were reported when using either a small (e.g., hand size of a primary school child) or a large (e.g., hand size of a tall man) rubber hand (Heed et al., 2011). Similarly, no significant differences in the illusion scores were detected when using a two dimensional hand image that was either equal in size, magnified or shrunk by $3 \mathrm{~cm}$ with respect to the participants' real hand (Pavani and Zampini, 2007). Similarly, the RHI was elicited with a small and a large rubber hand, still within the range of anatomically plausible body structures (Bruno and Bertamini, 2010). In contrast, using artificial body parts that violated normal body proportions, as for example a fake arm $91 \mathrm{~cm}$ beyond the real one, was shown to elicit weaker feelings of ownership through congruent visuotactile stimulation, compared to when the fake arm was seen with a plausible length (Armel and Ramachandran, 2003). When providing both visuotactile and visuomotor stimulation, participants gave high scores of ownership over a virtual arm up to three times the length of the real one, but less strongly at four times the length (Kilteni et al., 2012).

A similar effect was found when manipulating the number of artificial body parts. For example, stroking a rubber hand synchronously with the participants' visible hand (Guterstam et al., 2011), or stroking two rubber hands placed side-by-side synchronously with the participants' occluded real hand (Ehrsson, 2009), was shown to induce the illusion of having multiple limbs or owning two hands of the same laterality at the same time, respectively. Nevertheless, in both cases the experienced ownership was stronger when employing the classic RHI setup. With respect to simultaneous ownership toward two rubber hands of the same laterality, it was shown that these need to be placed symmetrically with respect to the real limb (Folegatti et al., 2012). High illusion scores concerning having two limbs were further reported when participants were moving their hand while seeing two video replicas, projected symmetrically with respect to the real, moving accordingly (Newport et al., 2010).

The spatial arrangement of the artificial body part with respect to the participant's body was instead demonstrated to be critical. For example, seeing a right rubber foot being stroked synchronously with the participants' right hand did not induce ownership feelings (Guterstam et al., 2011). Analogously, no significant differences were found in proprioceptive drifts when comparing synchronous and asynchronous stimulation applied on a right rubber hand and the participants' left hand (Tsakiris and Haggard, 2005). 


\subsubsection{The Role of Stimulation Congruence}

Another aspect of semantic congruence concerns the compatibility of the seen and felts touch when inducing BOls through visuotactile stimulation. In the majority of studies, the instrument seen to administer the touch on the artificial body was the same-e.g., paintbrushes (Botvinick and Cohen, 1998), sharp pins (Capelari et al., 2009), rods (Petkova and Ehrsson, 2008)—or a visually compatible one, with the one used to deliver the touch on the real body (Slater et al., 2008; Pabon et al., 2010; Normand et al., 2011; Maselli and Slater, 2013). Nevertheless, using different instruments could inhibit the occurrence of the $\mathrm{BOI}$ by violating the participants' tactile expectancies: the participant who sees the fake body being touched by a soft texture material, would expect sensations of analogous quality on his/her real body. This was investigated by using a piece of cotton to stimulate the rubber hand while using a piece of sponge to stimulate the real hand or vice versa. Results from both proprioceptive drifts and questionnaires were not significantly different from the case in which the same instrument was used (Schütz-Bosbach et al., 2009).

\subsubsection{SUMmARY}

Experimental evidence suggests that BOls are greatly affected by the semantic information the visual cues of the fake body bring along, at least in terms of their shape, the anatomical plausibility of their spatial configuration and structure. Even if some forms of anatomical violations (e.g., larger or smaller bodies, longer or multiple limbs) can be tolerated and overcome by congruent multimodal stimulation, other forms of violation (e.g., spatial arrangement) may not. This suggests that, in order to be perceived as parts of the own body, fake objects need to satisfy to some extent semantic constraints from an abstract and not self-specific body model (Tsakiris et al., 2007; Longo et al., 2009; Tsakiris, 2010). Yet, the exact boundaries of tolerable violations are still to be determined.

\section{Theoretical Accounts for Induction of Body OWNERSHIP ILLUSIONS}

The studies on BOls that we have reviewed so far provide important insights on the contribution of multisensory signals and semantic knowledge on the emergence of our sense of body ownership. However, in order to gain a deeper understanding on how the brain infers which is our body and its limbs, it is necessary to understand why BOls are induced at all. Although there is extensive consensus in the literature that multisensory processing of bodily signals underlies the induction of BOls (Graziano and Botvinick, 2002; Makin et al., 2008; Tsakiris, 2010; Ehrsson, 2011; Maselli and Slater, 2013), very few researchers attempted to cast the experimental conclusions into a unitary framework.

The initial Botvinick and Cohen's proposal was that the RHI arises from the three-way interaction between vision, touch and proprioception (Botvinick and Cohen, 1998). This idea was reflected in the proposal of Makin et al. (2008). According to their model, visual and proprioceptive cues about the positions of the real and rubber hands are first combined into a single estimate of the hand's spatial configuration; following, visual and tactile information about the seen and felt touches are processed 
in the common reference frame of the hand and, if integrated, elicit the illusion. This proposal was extended to account for experimental findings on the contribution of semantic information. According to the model proposed by Tsakiris (2010), the integration of multisensory cues is gated by semantic information in terms of the shape and anatomical plausibility of the seen object; only those objects that fit a pre-existing internal model of the human body can be therefore experienced as parts of the own body. In his account,Tsakiris proposed that this "test-for-fit" process takes place in the right temporo-parietal junction (Tsakiris et al., 2008). Alternatively, it was suggested that this selection process might be operated by visuoproprioceptive populations (Maselli and Slater, 2013).

Although these models offer a schematic description of the induction of BOls, by referring also to candidate brain areas that operate the multisensory integration and the semantic gating, they do not explain what is the underlying computational problem, and how our brain solves it with the resulting illusory experience. In the following, we briefly review recent predictive coding accounts that propose a specific computational framework for the study of self-processing, including the sense of body ownership. Subsequently, we focus on two alternative computational accounts, namely the Bayesian causal inference models and the connectionist models. Both approaches have been developed to explain various multisensory perceptual phenomena and they could be adopted and extended in future works to address the problem of BOls.

\subsection{Predictive Coding Approaches}

In a recent stream of work (Hohwy, 2007; Apps and Tsakiris, 2013; Clark, 2013; Limanowski and Blankenburg, 2013; Seth, 2013), the problem underpinning self-processing and self-recognition has been approached within the general framework of hierarchical generative models, such as predictive coding (Srinivasan et al., 1982; Rao and Ballard, 1999) and the free-energy principle (e.g., Friston, 2009). These models adopt the view, originally put forward by Von Helmholtz (Hatfield, 1990), that the brain needs to infer the hidden causes of the sensory signals; for example, the existence of a dog when hearing barking-like sound. It does so, by minimizing the error between the predictions it makes about the expected cues based on its internal causal model (e.g., what would be the auditory stimulus associated with a dog) and the available sensory information (e.g., the heard barking sound).

In this context it has been proposed that the $\mathrm{RHI}$ results from minimizing the prediction errors that arise from feeling the touch on the real hand and seeing the touch at a different location (i.e., on the rubber hand), as well as the errors concerning the rubber hand not looking exactly as the real hand. Error minimization would thus result in merging the spatial representation of the two hands and further in updating prior beliefs about one's own hand appearance (Apps and Tsakiris, 2013; Seth, 2013). Interestingly, several findings from RHI studies can be explained as the effect of strategies for error minimization; this is the case of the attenuation of somatosensory processing in the real hand, like e.g., the slowing of tactile temporal processing (Moseley et al., 2008), the increase of tactile detection threshold (Zopf et al., 2011), and the reduction of primary somatosensory cortex responses to tactile stimuli (Zeller et al., 2014). Since the main source of errors arises from the spatial discrepancies between the visual position of the rubber hand and the 
position of the real one provided by somatosensation, decreasing the precision of the latter could constitute an effective strategy to minimize the error (Zeller et al., 2014).

Despite the insights that these models provide about possible neurobiological mechanisms underlying BOls, to date these proposals have been formulated mainly at a conceptual level, without tackling the underlying computational problem.

\subsection{Causal Inference in Multimodal Cue Integration under BOls}

In contrast to predictive coding accounts, Bayesian causal inference models offer a simpler and comprehensive computational account for multisensory phenomena, usually without addressing the neurobiological mechanisms.According to these models, when people are presented with two stimuli from different modalities, they initially infer whether these have the same origin (i.e., cause) or not, and then they combine their information according to these beliefs (Körding et al., 2007). For example, when we hear a barking sound and we see a dog close to the auditory source, we will be confident that the barking sound comes from the seen dog and we will therefore use both the visual and the auditory information to estimate the position of the dog. In contrast, when the sound is perceived to come from a very distinct position with respect to the position of the seen dog, we may consider the existence of two dogs as more likely. Causal inference models were shown to explain various phenomena in multisensory perception (Shams and Beierholm, 2010), including the spatial (Körding et al., 2007; Wozny et al., 2010) and temporal (Shams et al., 2005) ventriloquist effects, and visuomotor adaptation (Körding and Tenenbaum, 2006;Wei and Körding, 2009).

While these models have been typically used to describe the combination of two sensory cues, analogous ideas could be applied to explain the induction of BOls. Indeed,Armel and Ramachandran (2003) proposed that the $\mathrm{RHI}$ is induced due to a Bayesian perceptual learning driven by the high likelihood of the visual and tactile stimuli to occur from one common event. In other words, it is more likely that the seen touch and the felt touch belong to the same event (i.e., my hand being stroked), than the existence of one artificial hand that is stroked synchronously with my hidden hand. Due to the fact that their proposal was exclusively based on the likelihood of sensory data, it was quickly put aside by other authors, as it was unable to explain the inhibition of the illusion under semantic violations (Tsakiris and Haggard, 2005; Tsakiris, 2010).

Nevertheless, a Bayesian model that includes semantic influences can be used to address the induction of RHI. For example, using the causal inference framework, the computational problem the brain needs to solve refers to inferring whether there is a common cause (i.e., my hand) or two different causes (i.e., my hand and the rubber one) generating the available visual, tactile, and proprioceptive signals (Figure 4). Mathematically formalizing the problem, the nervous system needs to calculate the probability of there being one hand $(C=1)$ vs. there being two hands $(C=2)$, given the available sensory information and prior knowledge. At time $\tau$ the sensory data consist of the static visual $\left(x_{v}\right)$ and proprioceptive $\left(x_{p}\right)$ spatial configuration of the real and rubber hands, the semantic information of the seen object $\mathrm{mv}$, and the trains of tactile stimuli applied, up to the current moment $\tau$, on the 
rubber $\left(s_{V} \rightarrow \tau\right)$ and the real $\rightarrow\left(s_{T} \rightarrow \tau\right)$, respectively (Equation 1).

$$
\mathrm{p}\left(\mathrm{C}=1 \mid \mathrm{m}_{\mathrm{V}}, \mathrm{x}_{\mathrm{V}}, \mathrm{x}_{\mathrm{P}}, \mathrm{s}_{\mathrm{V}}^{\tau}, \mathrm{s}_{\mathrm{T}}{ }^{\tau}\right)=\frac{\mathrm{p}\left(\mathrm{m}_{\mathrm{V}}, \mathrm{x}_{\mathrm{V}}, \mathrm{x}_{\mathrm{P}}, \vec{s}_{\mathrm{V}}^{\tau}, \mathrm{s}_{\mathrm{T}}^{\tau} \mid \mathrm{C}=1\right) \mathrm{p}(\mathrm{C}=1)}{\mathrm{p}\left(\mathrm{m}_{\mathrm{V}}, \mathrm{x}_{\mathrm{V}}, \mathrm{x}_{\mathrm{P}}, \mathrm{s}_{\mathrm{V}}^{\tau}, \mathrm{s}_{\mathrm{T}}^{\tau}\right)}
$$

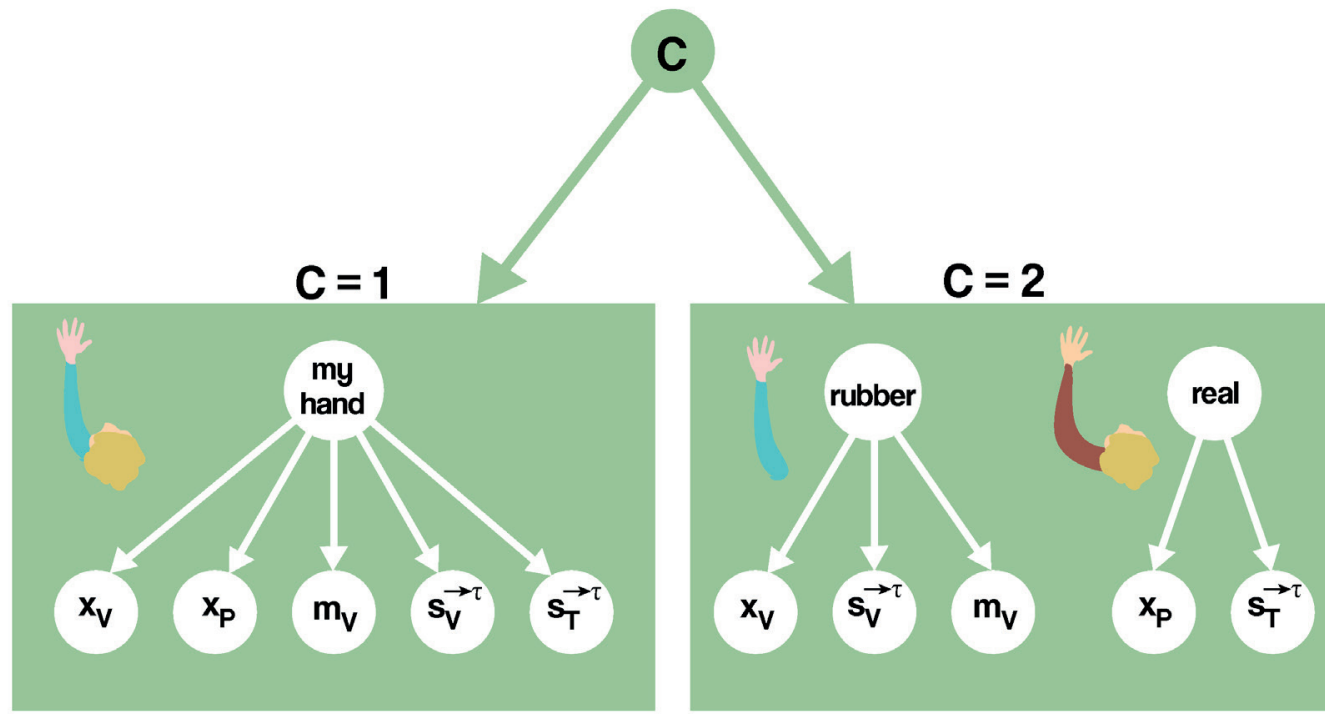

Figure 4. A causal inference model for the classic version of the $R H$ I. Left: one cause being responsible for all cues. In this case, the visually perceived configuration $x_{v}$ the proprioceptive perceived configuration $x_{p}$, the seen strokes $s_{V}{ }^{\rightarrow \tau}$ and the felt strokes $s_{T}{ }^{\rightarrow \tau}$ together with the seen morphological characteristics $m_{v}$, are mapped into a common cause $(C=1)$. Right: alternatively, two distinct causes may be inferred, decoupling the problem into two independent estimation problems. The brain infers whether the seen and felt spatial configurations, the tactile events and the seen morphology origin from the same causal structure, i.e., my hand $(C=1)$, or independent causal structures, i.e., the real and the rubber hands $(C=2)$, and then derives optimal predictions from this.

Under the simplifying assumption that the visual, tactile and proprioceptive information are conditionally independent, the problem can then be formulized as:

$\mathrm{p}\left(\mathrm{C}=1 \mid \mathrm{m}_{\mathrm{V}}, \mathrm{x}_{\mathrm{V}}, \mathrm{x}_{\mathrm{P}}, \mathrm{s}_{\mathrm{V}}^{\tau}, \mathrm{s}_{\mathrm{T}}^{\tau}\right)=\frac{\mathrm{p}\left(\mathrm{m}_{\mathrm{V}} \mid \mathrm{C}=1\right) \mathrm{p}\left(\mathrm{x}_{\mathrm{V}}, \mathrm{x}_{\mathrm{P}} \mid \mathrm{C}=1\right) \mathrm{p}\left(\vec{s}_{\mathrm{V}}^{\tau}, \mathrm{s}_{\mathrm{T}}^{\tau} \mid \mathrm{C}=1\right) \mathrm{p}(\mathrm{C}=1)}{\mathrm{p}\left(\mathrm{m}_{\mathrm{V}}, \mathrm{x}_{\mathrm{V}}, \mathrm{x}_{\mathrm{P}}, \overrightarrow{\mathrm{s}}_{\mathrm{V}}^{\tau}, \mathrm{s}_{\mathrm{T}}^{\tau}\right)}$

The model described by Equations (1) and (2) states that the probability for a single hand existing, estimated at time $\tau$ given the sensory evidence, depends on the likelihood of the evidence given 
only one hand $-\mathrm{p}\left(\mathrm{m}_{\mathrm{V}}, \mathrm{x}_{\mathrm{V}}, \mathrm{x}_{\mathrm{p}}, \mathrm{s}_{\mathrm{V}} \rightarrow \tau, \mathrm{s}_{T} \rightarrow \tau \mid \mathrm{C}=1\right)$ - and on the prior expectation for only one hand being there $-P(C=1)$. The likelihood of the data depends on (i) the distance between the two hands, i.e., $p\left(x_{v}, x_{p} \mid C=1\right)$, (ii) the spatiotemporal discrepancies of the visuotactile stimulation, i.e., $P\left(s_{V} \rightarrow \tau, s_{T} \rightarrow \tau \mid C=1\right)$, and (iii) the level of semantic congruence between the seen hand and the real one, i.e., $p\left(m_{v} \mid C=1\right)$. These visuoproprioceptive, visuotactile, and semantic factors contribute to the likelihood by increasing it in proportion to the congruency of the correspondent stimuli ${ }^{1}$. The likelihood, in turn, is in continuous interplay with prior expectations (e.g., the position of the limbs close to the body midline, personality traits including suggestibility to illusory perceptions, previous experience of the illusion), to update the posterior probability of only one hand being responsible for the sensory inputs, $\mathrm{p}\left(\mathrm{C}=1 \mid \mathrm{m}_{\mathrm{v}}, \mathrm{x}_{\mathrm{v}}, \mathrm{x}_{\mathrm{p}}, \mathrm{s}_{\mathrm{V}} \rightarrow \tau, \mathrm{s}_{\mathrm{T}} \rightarrow \tau\right)$. The illusion is then experienced when this posterior probability exceeds a given threshold (Wozny et al., 2010).

This relatively simple model, at least qualitatively, can accommodate many of the experimental findings on the RHI. For example, when the rubber hand is placed close to the real hand and without violating anatomical constraints, spatiotemporally congruent visuotactile stimulation will accumulate evidence in favor of a common hand being the source of all sensations and would induce the illusion (e.g., Botvinick and Cohen,1998). The progressive convergence of the model to a common hand scenario is also supported by experimental evidence that the RHI is not immediate but needs time to be elicited (Peled et al., 2000; Ehrsson et al., 2004; Perez-Marcos et al., 2012). On the other hand, when the visuotactile stimulation is asynchronous (e.g., Botvinick and Cohen, 1998;Armel and Ramachandran, 2003) or spatially incongruent (e.g., Kammers et al., 2009), the evidence will favor the existence of two hands, and the illusion will not be induced. In addition, semantic violations in terms of shape, anatomical plausibility and realism will render the contribution of the likelihood less important and therefore, the induction of the illusion more difficult, and its resulting strength weaker (Armel and Ramachandran, 2003; Haans et al., 2008; Ehrsson, 2009; Ide, 2013). Moreover, placing the rubber hand far from the real one and from the participant's midline corresponds to decreasing both the likelihood of visuoproprioceptive signals and the prior probability of the limb's position (Lloyd, 2007; Preston, 2013). Finally, when the two hands are overlapping or close in space, the high likelihood of visuoproprioceptive information can render the contribution of the visuotactile likelihood unnecessary (Giummarra et al., 2010) or less relevant (Zopf et al., 2010).

This formulation of the problem could be further extended to dynamically include the effects of having experienced the illusion at a given time, on the (prior) expectation for the illusion to occur at following times, as well as on the way the brain processes the incoming sensory signals (likelihood) later on. Computationally this could be done by adopting an iterative approach in which both the prior expectation and the probability densities representing the likelihoods at a given time step, evolve over time as a function of the posterior probability. Such an extension of the model could account for several aftereffects reported in the literature. In Hohwy and Paton (2010), for example, it was reported that if, after a period of congruent visuotactile stimulation in which the illusion is established, the stimulation switches to incongruent patterns (e.g., seeing elevated stroking on the rubber hand while receiving physical touch) then participants would keep experiencing the illusion together with unusual perceptual sensations (e.g., supernaturally caused tactile sensations). These results can indeed be explained by the fact that having experienced the illusion enhances the

1. The probability distribution functions in Equation (2) can be for example approximated as normal distributions in the correspondent metric. 
expectation for the illusion to be there (increasing the prior probability) and, at the same time, this modulates the probability densities of the likelihoods involved $\left(P\left(s_{V} \rightarrow \tau, s_{T} \rightarrow \tau \mid C=1\right)\right.$ in this case), e.g., increasing the variance, so to "explain away" the ongoing illusion and suppress the sensory conflict. This formulation is conceptually analogous to minimizing prediction errors in predictive coding approaches and, as discussed in the previous section, can account for several after-effects of the illusion, e.g., the attenuation of the somatosensory processing on the real hand (see the previous section for references).

It is noteworthy that this type of model can be adapted to include the effects of patterns of visuotactile stimulations that go beyond spatiotemporal congruency, such as applied pressure, duration, trajectory, frequency, velocity or predictability. Recent studies have observed that some of these features, closely related to affective and emotional aspects of touch, (e.g., the velocity of the applied stimulation) can significantly modulate the RHI (Crucianelli et al., 2013; Lloyd et al., 2013; van Stralen et al., 2014). Particularly concerning the predictability of the visuotactile stimulation, the probability of two independent visuotactile patterns being spatiotemporally congruent is lower the higher their complexity. Therefore, being exposed to congruent patterns of increasing complexity should lead to stronger illusions, and/or to illusions established on shorter time scales. Although there is no study explicitly testing this, reports in the literature support that increasing the stimulation variability can avoid habituation effects and lead to a faster and stronger illusion (Niebauer et al., 2002; Armel and Ramachandran, 2003; Tsakiris et al., 2008; Petkova and Ehrsson, 2009; Guterstam et al., 2011). The same observations were reported for visuomotor stimulation (Tsakiris et al., 2006; Kalckert and Ehrsson, 2012). This evidence is consistent with the proposal that unpredictability increases the weight of multisensory likelihood in inferring a common body.

A similar causal inference model can be applied to address the induction of BOls in presence of visuomotor stimuli. In this case, visual and proprioceptive inputs that change dynamically in time can provide accumulating evidence in favor of a single cause if congruent $-p\left(C=1 \mid m_{v}, x_{v} \overrightarrow{ }, x_{p} \overrightarrow{ }\right)$. Such a formulation can further bring forward the question of whether some crossmodal stimuli contribute more in inferring our own body than others. Congruent visuotactile or visuomotor stimuli between the real and rubber index finger was shown to elicit the RHI without significant differences (Riemer et al., 2013; Kalckert and Ehrsson, 2014a). Nevertheless, when testing the relative role of visuomotor and visuotactile correlations between the real and the virtual lower limbs under the same experimental condition, a stronger contribution of visuomotor stimuli was found in inducing the $\mathrm{BO}$, whilst incongruent stimulation of each of the two led equally to its break (Kokkinara and Slater, 2014). Given that visuomotor correlations contain a larger amount of information compared to visuotactile correlations (e.g., in terms of the internal model involved), the proposed model would predict a faster onset of BOls. Moreover, this facilitation might also depend on which body parts are involved in the movements (e.g., moving the index finger vs. moving an entire upper or lower limb). However, these predictions need to be explicitly tested.

Finally, the same model could be reformulated and extended to account for the full BOls taking into account the relevant pieces of multimodal and semantic information. 


\subsection{Network Models}

Connectionist models offer an alternative approach for addressing the problem of BOls. Although they have not been previously envisioned in this context, these models have been successfully applied in the field of multisensory integration as an alternative approach to Bayesian models (e.g., Patton and Anastasio, 2003; Martin et al., 2009; Cuppini et al., 2011). Connectionist models implement artificial networks with architectures that are typically inspired from neurobiological structures and are suited to simulate learning mechanisms and collective neuronal behavior.They therefore offer an important advantage with respect to the Bayesian approaches, as they can provide deeper insights into the neural mechanisms involved in the processes under study, although at the expense of higher complexity (Fernandes and Kording, 2010)

Apart from reproducing some of the most relevant properties of multisensory processing at the level of neural activity (Patton and Anastasio, 2003; Rowland et al., 2007; Martin et al., 2009), recent implementations have been proposed to account for perceptual illusions. For example, relatively simple networks, consisting of two layers reciprocally interconnected, could reproduce common illusions such as the sound-induced flash illusion and fusion (Cuppini et al., 2014), as well as the ventriloquist effect and aftereffect (Magosso et al., 2012).

Particularly relevant for the present discussion is the implementation of neural networks whose dynamics reproduces several observed properties of visuotactile processing. For example, a network with two unimodal and one bimodal areas, could reproduce effects of visuotactile integration such as the facilitation of tactile detection, localization and acuity, by concurrent visual information (Magosso, 2010). Modifications of this implementation have been shown to reproduce other key aspects of visuotactile integration. For example, by including Hebbian learning mechanisms tuned by attentional effects (Ursino et al., 2007; Magosso et al., 2010b), it was possible to simulate the dynamical expansion of the peripersonal space observed after tool use (Maravita and Iriki, 2004). In addition, interhemispheric competition effects, similar to the ones observed in right-brain-damaged patients (di Pellegrino et al., 1997; Mattingley et al., 1997), have been further emulated with models in which two replications of the network described above (one for each hand) were interconnected by inhibitory interneuron modules (Magosso et al., 2010a,b).

All these remarkable results address the possibility to apply this class of model to develop new theoretical accounts for BOls. A plausible scenario would be to initially combine these models with Bayesian modules. For example, while the visuotactile processing involved in the RHI could be simulated with neural networks, the test-for-fit gating associated with neural populations specialized in body parts recognition, could be more conveniently described with the Bayesian formalism. Alternatively, existing implementations of neural networks for the representation of semantic information (for a review see Ursino et al., 2014), provide a valid option for representing sematic factors in BOls. Such envisioned hybrid models would be indeed ideal for gaining deeper insights into our current understanding of the BOls and their implications for own-body perception. 


\section{Conclusions}

One of the important questions in neuroscience, psychology, and philosophy concerns how we distinguish our body from the bodies of others as well as from objects in the surrounding environment (Gallagher, 2000; Jeannerod, 2003; Blanke and Metzinger, 2009; de Vignemont, 2011). Body ownership illusions (BOIs) are a powerful experimental tool to address this question, since they permit to investigate the conditions under which we can perceive artificial body parts or fake bodies as belonging to ourselves. In the present review, we focused on the role of multisensory integration and semantic knowledge in inducing BOls. In particular, we reviewed the role of the temporal, spatial, and semantic relationship of crossmodal stimuli in the elicitation of the illusions as reflected in several experimental studies, and we further discussed and proposed theoretical accounts in order to cast all this information into one computational framework.

Given the vastness of the experimental literature around BOls, the present review is not exhaustive. For example, we focused on BOls induced in presence of visual information, excluding therefore the somatic versions of BOls and similar body illusions induced with auditory cues (TajaduraJiménez et al., 2012). Nevertheless, similar principles to those proposed here have been revealed: e.g., temporal constraints in visuotactile integration (Ehrsson et al., 2005a) and negligible effects of tactile quality (White et al., 2010). In addition, we did not address the important contribution of interoceptive signals in the sense of body ownership (Seth, 2013), although this has recently shown to influence different aspects of BOls (Crucianelli et al., 2013; Lloyd et al., 2013; van Stralen et al., 2014).

Moreover, our theoretical proposals based on computational principles that combine and integrate exteroceptive and proprioceptive signals for explaining the induction of BOls, do not imply that the emergence of the sense of body ownership relies on a strictly deterministic computation. For example, interoceptive signals and emotional states are fundamental in our sense of embodiment (Carruthers, 2008; Seth, 2013). Nevertheless, we consider that formulating and experimentally validating computational models, even if these are approximate and not exhaustive, can be a first step toward deciphering the mechanisms underlying BOls. These approaches can be extended to include further contributions and to account for a more holistic view of our own body-perception.

Beside the necessity for experimental validation of such theoretical models, future studies are needed to investigate whether and how the contribution of spatial, temporal, and semantic characteristics of crossmodal stimuli changes once BOls are experienced.That is, while we discussed the experimental principles in the context of inducing the illusions, there is the possibility that these spatial, temporal, and semantic constraints are adapted after the onset of the illusion-a possibility discussed above as part of the causal model.The study of Hohwy and Paton (2010) points certainly in this direction: once the rubber hand illusion was induced with a standard paradigm, the illusion could be sustained even when suddenly introducing semantic or spatiotemporal violations in the crossmodal stimuli. The result of this intervention was the elicitation of unusual experiences such as tactile sensations originating from a stroked cardboard, or supernatural tactile sensations generated by an elevated finger that was not in contact with the rubber hand. Similarly, in the study 
of Kilteni et al. (2012) the gradual introduction of semantic violations in terms of body proportions did not abolish but sustained the ownership illusion toward a very long fake arm. The same question could be also applied to the temporal aspects of visuotactile integration; for example, does the illusion persist if the administered visuotactile stimuli progressively change from synchronous to asynchronous? Indeed, there is evidence suggesting that a full $\mathrm{BO}$ triggered by seeing a realistic virtual body in spatial coincidence with the physical body, could be sustained during asynchronous visuotactile stimulation (Maselli and Slater, 2013).

Last but not least, the present review leaves the question of why somatoparaphrenic patients perceive their body parts as not belonging to themselves, unanswered. Although outside the scope of the present review, recent studies have started to explore the induction of BOls with somatoparaphrenic patients (Jenkinson et al., 2013; van Stralen et al., 2013; Bolognini et al., 2014). Investigating whether or not the principles on multimodal triggers and semantic information mentioned here for healthy subjects, apply also for these patients, could provide a possible strategy to grasp the link between delusions and illusions of body ownership. 


\section{ReferenCES}

Aimola Davies, A. M., White, R. C., and Davies, M. (2013). L. T. (2011). The rubber hand illusion in a mirror. Conscious. Spatial limits on the nonvisual self-touch illusion and the visual Cogn. 20,1108-1119

rubber hand illusion: subjective experience of the illusion and proprioceptive drift. Conscious. Cogn. 22, 613-636

Bertamini, M., and O'Sullivan, N. (2014). The use of realistic and mechanical hands in the rubber hand illusion, and the Alais, D., Newell, F.N., and Mamassian, P.(2010). Multisensory relationship to hemispheric differences. Conscious. Cogn. 27, processing in review: from physiology to behaviour. Seeing 89-99

Perceiving 23, 3-38

Blanke, O. (2012). Multisensory brain mechanisms of bodily Altschuler, E. L., and Ramachandran, V. S. (2007). A simple self-consciousness. Nat. Rev. Neurosci. 13, 556-571 method to stand outside oneself. Perception 36, 632-634.

Blanke, O., and Metzinger, T. (2009). Full-body illusions and Apps, M. A. J., and Tsakiris, M. (2013). The free-energy self: minimal phenomenal selfhood.Trends Cogn. Sci. 13, 7-13 a predictive coding account of self-recognition. Neurosci. Biobehav. Rev. 41, 85-97

Blanke, O., and Mohr, C. (2005). Out-of-body experience, heautoscopy, and autoscopic hallucination of neurological Armel, K. C., and Ramachandran, V. S. (2003). Projecting origin implications for neurocognitive mechanisms of corporeal sensations to external objects: evidence from skin conductance awareness and self-consciousness. Brain Res. Brain Res. Rev. response. Proc. Biol. Sci. 270, 1499-1506

50, 184-199

Azañón, E., and Soto-Faraco, S. (2007). Alleviating the Bolognini, N., Ronchi, R., Casati, C., Fortis, P., and Vallar, G. "crossed-hands" deficit by seeing uncrossed rubber hands. Exp. (2014). Multisensory remission of somatoparaphrenic delusion: Brain Res. 182, 537-548 my hand is back! Neurol. Clin. Pract. 4, 216-225

Bagesteiro, L. B., Sarlegna, F. R., and Sainburg, R. L. (2006). Botvinick, M., and Cohen, J. (1998). Rubber hands "feel” touch Differential influence of vision and proprioception on control of that eyes see. Nature 391:756 movement distance. Exp. Brain Res. 171, 358-370

Boulinguez, P., and Rouhana, J. (2008). Flexibility and individual Bahrick, L. E., and Watson, J.S. (1985). Detection of intermodal differences in visuo-proprioceptive integration: evidence from the proprioceptive-visual contingency as a potential basis of self- analysis of a morphokinetic control task. Exp. Brain Res. 185, perception in infancy. Dev. Psychol. 21, 963-973 137-149

Banakou, D., Groten, R., and Slater, M. (2013). Illusory Bremner, A. J., Hill, E. L., Pratt, M., Rigato, S., and Spence, C. ownership of a virtual child body causes overestimation of object (2013). Bodily illusions in young children: developmental change sizes and implicit attitude changes. Proc. Natl.Acad.Sci.U.S.A. in visual and proprioceptive contributions to perceived hand $110,12846-12851$ position. PLoS ONE 8:e51887

Battaglia-Mayer, A., Ferraina, S., Mitsuda, T., Genovesio, Brozzoli, C., Gentile, G., and Ehrsson, H. H. (2012). That's A., Onorati, P., Lacquaniti, F., et al. (2000). Early coding of near my hand! Parietal and premotor coding of hand-centered reaching in the parietooccipital cortex early coding of reaching space contributes to localization and self-attribution of the hand. in the parietooccipital cortex. J. Neurophysiol. 83, 2374-2391 J. Neurosci. 32, 14573-14582

Bernardi, N. F., Marino, B. F., Maravita, A., Castelnuovo, G., Bruno, N., and Bertamini, M. (2010). Haptic perception after a Tebano, R., and Bricolo, E. (2013). Grasping in wonderland: change in hand size. Neuropsychologia 48, 1853-1856 altering the visual size of the body recalibrates the body schema.

Exp. Brain Res. 226, 585-594.

Burke, B. Y. D., Gandevia, S. C., and Macefield, G. (1988). Responses to passive movement of receptors in joint, skin and Bertamini, M., Berselli, N., Bode, C., Lawson, R., and Wong, muscle of the human hand. J. Physiol. 402, 347-361 
illusions modulate tactile perception. Curr. Biol. 15, 1286-1290

Butz, M. V., Kutter, E. F., and Lorenz, C. (2014). Rubber hand illusion affects joint angle perception. PLoS ONE 9:e92854

di Pellegrino, G., Làdavas, E., and Farnè, A. (1997). Seeing where your hands are. Nature 388:730

Cadieux, M. L., Whitworth, K., and Shore, D. I. (2011). Rubber hands do not cross the midline. Neurosci. Lett. 504, 191-194

Doehrmann, O., and Naumer, M. J. (2008). Semantics and the multisensory brain: how meaning modulates processes of audioCapelari, E.D.P.,Uribe, C., and Brasil-Neto, J.P.(2009). Feeling visual integration. Brain Res. 1242, 136-150 pain in the rubber hand: integration of visual, proprioceptive, and painful stimuli. Perception 38, 92-99.

Duhamel, J., Colby, C. L., and Goldberg, M. E. (1998). Ventral intraparietal area of the macaque: congruent visual and somatic Carruthers, G. (2008). Types of body representation and the response properties. J. Neurophysiol. 79, 126-136 sense of embodiment. Conscious. Cogn. 17,1302-1316

Dummer,T., Picot-Annand,A., Neal,T., and Moore, C. (2009). Clark, A. (2013). Whatever next? Predictive brains, situated Movement and the rubber hand illusion. Perception 38, 271agents, and the future of cognitive science. Behav. Brain Sci. 36, 280

181-204

Ehrsson, H. H. (2007). The experimental induction of out-ofCostantini, M., and Haggard, P. (2007). The rubber hand body experiences. Science 317, 1048. illusion: sensitivity and reference frame for body ownership. Conscious. Cogn. 16, 229-240

Ehrsson, H. H. (2009). How many arms make a pair? Perceptual illusion of having an additional limb. Perception 38, 310-312

Crucianelli, L., Metcalf,N.K., Fotopoulou,A. K., and Jenkinson, P.M. (2013). Bodily pleasure matters: velocity of touch modulates Ehrsson, H. H. (2011). "The concept of body ownership and its body ownership during the rubber hand illusion. Front. Psychol. relation to multisensory integration," in The New Handbook 4:703 of Multisensory Processes, ed B. E. Stein (Cambridge: MIT Press), 775-792

Cuppini, C., Magosso, E., Bolognini, N.,Vallar, G., and Ursino, M. (2014). A neurocomputational analysis of the sound-induced Ehrsson, H. H., Holmes, N. P., and Passingham, R. E. (2005a). flash illusion. Neuroimage 92, 248-266

Cuppini, C., Magosso, E., and Ursino, M. (2011). Organization, maturation, and plasticity of multisensory integration: insights from computational modeling studies. Front. Psychol. 2:77

Touching a rubber hand: feeling of body ownership is associated with activity in multisensory brain areas. J. Neurosci. 25, 10564-

Daprati, E., Franck, N., Georgieff, N., Proust, J., Pacherie, E., Dalery,J., et al.(1997). Looking for the agent:an investigation into Ehrsson, H. H., Spence, C., and Passingham, R. E. (2004). consciousness of action and self-consciousness in schizophrenic That's my hand! Activity in premotor cortex reflects feeling of patients. Cognition 65, 71-86 ownership of a limb. Science 305, 875-877

Davies, P. (1973). Effects of movements upon the appearance Ehrsson, H. H., Wiech, K., Weiskopf, N., Dolan, R. J., and and duration of a prolonged visual afterimage: 1. Changes arising Passingham, R. E. (2007). Threatening a rubber hand that you from the movement of a portion of the body incorporated in the feel is yours elicits a cortical anxiety response. Proc. Natl.Acad. afterimaged scene. Perception 2,147-153

Sci. U. S.A. 104, 9828-9833

de Vignemont, F. (2011). Embodiment, ownership and Ernst, M. O., and Banks, M. S. (2002). Humans integrate visual disownership. Conscious. Cogn. 20, 82-93 and haptic information in a statistically optimal fashion. Nature 415, 429-433

deVignemont, F., Ehrsson, H. H., and Haggard,P.(2005). Bodily 
Evans, N., and Blanke, O. (2013). Shared electrophysiology 1382-1384

mechanisms of body ownership and motor imagery.

Neuroimage 64, 216-228

Graziano, M. S. A., and Botvinick, M. (2002). "How the brain represents the body: insights from neurophysiology and Feinberg, T. E., Venneri, A., Simone, A. M., Fan, Y., and psychology," in Common Mechanisms in Perception and Northoff, G. (2010). The neuroanatomy of asomatognosia Action: Attention and Performance, eds W. Prinz and B. and somatoparaphrenia. J. Neurol. Neurosurg. Psychiatry 81, Hommel (Oxford: Oxford University Press), 136-157 276-281

Graziano, M. S. A., and Cooke, D. F. (2006). ParietoFernandes, H. L., and Kording, K.P. (2010). In praise of "false" frontal interactions, personal space, and defensive behavior. models and rich data. J. Mot. Behav. 42, 343-349 Neuropsychologia 44, 2621-2635

Filimon, F., Nelson, J. D., Huang, R.-S., and Sereno, M. I. Graziano, M. S. A., Cooke, D. F., and Taylor, C. S. (2000). (2009). Multiple parietal reach regions in humans: cortical Coding the location of the arm by sight. Science 290, 1782representations for visual and proprioceptive feedback during 1786

on-line reaching. J. Neurosci. 29, 2961-2971

Graziano, M. S.A., and Gross, C. G. (1993). A bimodal map of Fogassi, L., Gallese,V., Fadiga, L., Luppino, G., Matelli, M., and space: somatosensory receptive fields in the macaque putamen Rizzolatti, G. (1996). Coding of peripersonal space in inferior with corresponding visual receptive fields. Exp. brain Res. 97, premotor cortex (area F4). J. Neurophysiol. 76, 141-157 96-109

Folegatti, A., Farnè, A., Salemme, R., and de Vignemont, F. Graziano,M.S.A., and Gross, C.G.(1994). "The representation (2012). The rubber hand illusion: two's a company, but three's a of extrapersonal space: a possible role for bimodal, visuocrowd. Conscious. Cogn. 21, 799-812 tactile neurons," in The Cognitive Neurosciences, ed M. S. Gazzaniga (Cambridge, MA: MIT Press), 1021-1034.

Franck, N., Farrer, C., Georgieff, N., Marie-Cardine, M.,

Daléry, J., D'Amato, T., et al. (2001). Defective recognition Gregory, R. L., Wallace, J. G., and Campbell, F. W. (1959). of one's own actions in patients with schizophrenia. Am. J. Changes in the size and shape of visual after-images observed Psychiatry 158, 454-459 in complete darkness during changes of position in space. Q.J. Exp. Psychol. 11, 54-55

Friston, K. (2009). The free-energy principle: a rough guide to the brain? Trends Cogn. Sci. 13, 293-301.

Guterstam, A., Petkova, V. I., and Ehrsson, H. H. (2011). The illusion of owning a third arm. PLoS ONE 6:e17208

Gallagher, I. (2000). Philosophical conceptions of the self: implications for cognitive science.Trends Cogn. Sci. 4,14-21 Haans, A., ljsselsteijn, W. A, and de Kort, Y.A.W. (2008). The effect of similarities in skin texture and hand shape on perceived Gentile, G., Petkova,V.I., and Ehrsson, H.H.(2011). Integration ownership of a fake limb. Body Image 5, 389-394 of visual and tactile signals from the hand in the human brain: an FMRI study. J. Neurophysiol. 105, 910-922

Haggard, P., and Jundi, S. (2009). Rubber hand illusions and size - weight illusions: self-representation modulates representation Giummarra, M. J., Georgiou-Karistianis, N., Nicholls, M. E. of external objects. Perception 38,1796-1803

R., Gibson, S. J., and Bradshaw, J. L. (2010). The phantom in the mirror: a modified rubber-hand illusion in amputees and Hatfield, G. C. (1990). The Natural and the Normative:Theories normals. Perception 39, 103-118 of Spatial Perception from Kant to Helmholtz. Cambridge, MA: MIT Press

Gliga, T., and Dehaene-Lambertz, G. (2005). Structural encoding of body and face in human infants and adults. J. Cogn. Haxby, J., Hoffman, E., and Gobbini, M. (2000). The distributed Neurosci. 17, 1328-1340 human neural system for face perception. Trends Cogn. Sci. 4, 223-233

Goodwin, G. M., McCloskey, D. I., and Matthews, P. B. C. (1972). Proprioceptive illusions induced by muscle vibration: Hay, J., Pick, H., and lkeda, K. (1965). Visual capture produced contribution by muscle spindles to perception? Science 175, by prism spectacles. Psychon. Sci. 2, 215-216 
Insights from somatoparaphrenia and the rubber hand illusion.

Heed, T., Gründler, M., Rinkleib, J., Rudzik, F. H., Collins, T., Neuropsychologia, 1-10

Cooke, E., et al. (2011). Visual information and rubber hand

embodiment differentially affect reach-to-grasp actions. Acta Kalckert,A., and Ehrsson, H. H. (2012). Moving a rubber hand

Psychol. (Amst). 138, 263-271

that feels like your own: a dissociation of ownership and agency. Front. Hum. Neurosci. 6:40

Helbig, H. B., Ernst, M. O., Ricciardi, E., Pietrini, P., Thielscher,

A., Mayer, K. M., et al. (2012). The neural mechanisms of Kalckert, A., and Ehrsson, H. H. (2014a). The moving rubber reliability weighted integration of shape information from vision hand illusion revisited: comparing movements and visuotactile and touch. Neuroimage 60,1063-1072

stimulation to induce illusory ownership. Conscious. Cogn. 26, 117-132

Hirai, M., and Hiraki, K. (2005). An event-related potentials study of biological motion perception in human infants. Brain Res. Cogn. Brain Res. 22, 301-304

Kalckert, A., and Ehrsson, H. H. (2014b). The spatial distance rule in the moving and classical rubber hand illusions. Conscious. Cogn. 30C, 118-132

Hohwy, J. (2007). The sense of self in the phenomenology of agency and perception. Psyche (Stuttg). 13, 1-20. Available Kammers, M. P. M., Longo, M. R., Tsakiris, M., Dijkerman, H. online at: https://wwwpsyche.org/files/0xab11.pdf

C., and Haggard, P. (2009). Specificity and coherence of body representations. Perception 38,1804-1820

Hohwy, J., and Paton, B. (2010). Explaining away the body: experiences of supernaturally caused touch and touch on non- Kilteni, K., Bergstrom, I., and Slater, M. (2013). Drumming in hand objects within the rubber hand illusion. PLoS ONE 5:e9416. immersive virtual reality: the body shapes the way we play. IEEE Trans.Vis. Comput. Graph. 19, 597-605

Holle, H., McLatchie, N., Maurer, S., and Ward, J. (2011).

Proprioceptive drift without illusions of ownership for rotated Kilteni, K., Normand, J.-M., Sanchez-Vives, M. V., and Slater, hands in the "rubber hand illusion" paradigm. Cogn. Neurosci. M. (2012). Extending body space in immersive virtual reality: a 2, 171-178 very long arm illusion. PLoS ONE 7:e40867

Holmes, N. P., Crozier, G., and Spence, C. (2004). When Kokkinara, E., and Slater, M. (2014). Measuring the effects mirrors lie: "visual capture" of arm position impairs reaching through time of the influence of visuomotor and visuotactile performance. Cogn. Affect. Behav. Neurosci. 4, 193-200 synchronous stimulation on a virtual body ownership illusion. Perception 43, 43-58

Ide, M. (2013). The effect of "anatomical plausibility" of hand angle on the rubber-hand illusion. Perception 42,103-111

Körding, K.P., Beierholm, U., Ma,W. J., Quartz, S., Tenenbaum, J. B., and Shams, L. (2007). Causal inference in multisensory ljsselsteijn, W. A., de Kort, Y. A. W., and Haans, A. (2006). Is perception. PLoS ONE 2:e943

this my hand I see before me? The rubber hand illusion in reality, virtual reality, and mixed reality. Presence Teleoperators Körding, K.P., and Tenenbaum, J.B. (2006). "Causal inference in Virtual Environ. 15, 455-464 sensorimotor integration,” in Advances in Neural Information Processing Systems 19, eds B. Schölkopf, J. C. Platt, and T. lonta, S., Sforza, A., Funato, M., and Blanke, O. (2013). Hoffman (Cambridge, MA: MIT Press), 737-744.

Anatomically plausible illusory posture affects mental rotation of body parts. Cogn. Affect. Behav. Neurosci. 13, 197-209.

Lacey, S., Hall, J., and Sathian, K. (2010). Are surface properties integrated into visuo-haptic object representations? Eur. J. Jeannerod, M. (2003). The mechanism of self-recognition in Neurosci. 31, 1882-1888 humans. Behav. Brain Res. 142,1-15

Lackner, J. R. (1988). Some proprioceptive influences on the Jeannerod, M. (2004). Visual and action cues contribute to the perceptual representation of body shape and orientation. Brain self-other distinction. Nat. Neurosci. 7, 422-423 $111,281-297$

Jenkinson, P. M., Haggard, P., Ferreira, N. C., and Fotopoulou, Lackner, J. R., and Levine, M. S. (1978). Visual direction A. (2013). Body ownership and attention in the mirror: depends on the opertion of spatial constancy mechanisms: the 
oculobrachial illusion. Neurosci. Lett. 7, 207-212

Longo, M. R., Schüür, F., Kammers, M. P. M., Tsakiris, M., and Lajoie,Y., Paillard, J., Teasdale, N., Bard, C., Fleury, M., Forget, Haggard, P. (2009). Self awareness and the body image. Acta R., et al. (1992). Mirror drawing in a deafferented patient and Psychol. (Amst). 132, 166-172 normal subjects: visuoproprioceptive conflict. Neurology 42, 1104-1106

Magosso, E. (2010). Integrating information from vision and touch: a neural network modeling study. IEEE Trans. Inf.

Lenggenhager, B., Tadi, T., Metzinger, T., and Blanke, O. Technol. Biomed. 14, 598-612

(2007). Video ergo sum: manipulating bodily self-consciousness.

Science 317, 1096-1099

Magosso, E., Cuppini, C., and Ursino, M. (2012). A neural network model of ventriloquism effect and aftereffect. PLoS

Limanowski, J., and Blankenburg, F. (2013). Minimal selfmodels and the free energy principle. Front. Hum. Neurosci. 7:547

Magosso, E., Serino, A., di Pellegrino, G., and Ursino, M. (2010a). Crossmodal links between vision and touch in spatial Limanowski, J., Lutti, A., and Blankenburg, F. (2013). The attention: a computational modelling study. Comput. Intell. extrastriate body area is involved in illusory limb ownership. Neurosci. 2010:304941

Neuroimage 86, 514-524

Magosso, E., Ursino, M., di Pellegrino, G., Làdavas, E., and Linkenauger, S.A., Bülthoff, H. H., and Mohler, B. J. (in press). Serino, A. (2010b). Neural bases of peri-hand space plasticity Virtual arm's reach influences perceived distances but only after through tool-use: insights from a combined computationalexperience reaching. Neuropsychologia, 1-9 experimental approach. Neuropsychologia 48, 812-830

Linkenauger, S. A., Leyrer, M., Bülthoff, H. H., and Mohler, Makin, T. R., Holmes, N. P., and Ehrsson, H. H. (2008). On B. J. (2013). Welcome to wonderland: the influence of the size the other hand: dummy hands and peripersonal space. Behav. and shape of a virtual hand on the perceived size and shape of Brain Res. 191,1-10

virtual objects. PLoS ONE 8:e68594

Makin, T. R., Holmes, N. P., and Zohary, E. (2007). Is that near Llobera, J., Sanchez-Vives, M. V., and Slater, M. (2013). The my hand? Multisensory representation of peripersonal space in relationship between virtual body ownership and temperature human intraparietal sulcus. J. Neurosci. 27, 731-740 sensitivity. J. R. Soc. Interface 10:20130300

Maravita,A., and Iriki, A. (2004). Tools for the body (schema). Lloyd, D. M. (2007). Spatial limits on referred touch to an alien Trends Cogn. Sci. 8, 79-86 limb may reflect boundaries of visuo-tactile peripersonal space surrounding the hand. Brain Cogn. 64, 104-109

Martin, J. G., Meredith, M.A., and Ahmad, K. (2009). Modeling multisensory enhancement with self-organizing maps. Front.

Lloyd, D. M., Gillis, V., Lewis, E., Farrell, M. J., and Morrison, Comput. Neurosci. 3:8

I. (2013). Pleasant touch moderates the subjective but not objective aspects of body perception. Front. Behav. Neurosci. Maselli,A., and Slater, M. (2013). The building blocks of the full 7:207 body ownership illusion. Front. Hum. Neurosci. 7:83

Lloyd, D. M., Shore, D. I., Spence, C., and Calvert, G. A. Maselli, A., and Slater, M. (2014). Sliding perspectives: (2003). Multisensory representation of limb position in human dissociating ownership from self-location during full body premotor cortex. Nat. Neurosci. 6, 17-18 illusions in virtual reality. Front. Hum. Neurosci. 8:693

Longo, M. R., Cardozo, S., and Haggard, P. (2008a). Visual Mattingley, J. B., Driver, J., Beschin, N., and Robertson, I. H. enhancement of touch and the bodily self. Conscious. Cogn. (1997). Attentional competition between modalities: extinction $17,1181-1191$ between touch and vision after right hemisphere damage. Neuropsychologia 35, 867-880

Longo, M. R., Schüür, F., Kammers, M. P. M., Tsakiris, M., and Haggard, P. (2008b). What is embodiment? A psychometric Miall, R. C., and Wolpert, D. M. (1996). Forward models for approach. Cognition 107, 978-998 physiological motor control. Neural Networks 9,1265-1979 
T.-Q., and Ehrsson, H. H. (2011a). From part- to whole-body Moseley, G. L., Olthof, N., Venema, A., Don, S., Wijers, M., ownership in the multisensory brain. Curr. Biol. 21, 1118-1122 Gallace, A., et al. (2008). Psychologically induced cooling of a specific body part caused by the illusory ownership of an artificial Petkova,V.I., and Ehrsson, H.H.(2008). If I were you:perceptual counterpart. Proc. Natl.Acad. Sci. U.S.A. 105, 13169-13173 illusion of body swapping. PLoS ONE 3:e3832

Newport, R., Pearce, R., and Preston, C. (2010). Fake hands Petkova, V. I., and Ehrsson, H. H. (2009). When right feels left: in action: embodiment and control of supernumerary limbs. Exp. referral of touch and ownership between the hands. PLoS ONE brain Res. 204, 385-395 4:e6933

Niebauer, C.L.,Aselage, J., and Schutte,C.(2002). Hemispheric Petkova, V. I., Khoshnevis, M., and Ehrsson, H. H. (2011b). interaction and consciousness: degree of handedness predicts the The perspective matters! Multisensory integration in ego-centric intensity of a sensory illusion. Laterality 7, 85-96 reference frames determines full-body ownership. Front. Psychol. 2:35

Normand, J.-M., Giannopoulos, E., Spanlang, B., and Slater, M. (2011). Multisensory stimulation can induce an illusion of larger Pick, H. L., Hay, J. C., and College, S. (1969). Sensory conflict in belly size in immersive virtual reality. PLoS ONE 6:e16128 judgments of spatial direction. Percept. Psychophys. 6, 203-205

Pabon, S., Padilla, M.A., Frisoli,A., Greco, G., and Bergamasco, Preston, C. (2013). The role of distance from the body and M. (2010). Ownership illusion of the hand/arm through dynamic distance from the real hand in ownership and disownership during VR interactions and automatic vibrotactile stimulation (Viareggio: the rubber hand illusion. Acta Psychol. (Amst). 142, 177-183 RO-MAN, 2010 IEEE), 715-720

Ramachandran, V. S., and Hirstein, W. (1998). The perception Patton, P. E., and Anastasio, T. J. (2003). Modeling cross-modal of phantom limbs. Brain 121, 1603-1630 enhancement and modality-specific suppression in multisensory neurons. Neural Comput. 15, 783-810

Rao, R. P., and Ballard, D. H. (1999). Predictive coding in the visual cortex: a functional interpretation of some extra-classical Pavani, F., Spence, C., and Driver, J. (2000). Visual capture of receptive-field effects. Nat. Neurosci. 2, 79-87 touch: out-of-the-body experiences with rubber gloves. Psychol. Sci. 11, 353-359

Reid, V. M., Hoehl, S., and Striano, T. (2006). The perception of biological motion by infants: an event-related potential study.

Pavani, F., and Zampini, M. (2007). The role of hand size in the Neurosci. Lett. 395, 211-214

fake-hand illusion paradigm. Perception 36,1547-1554

Riemer, M., Fuchs, X., Bublatzky, F., Kleinböhl, D., Hölzl, R., Peck, T. C., Seinfeld, S., Aglioti, S. M., and Slater, M. (2013). and Trojan, J. (2014). The rubber hand illusion depends on a Putting yourself in the skin of a black avatar reduces implicit congruent mapping between real and artificial fingers. Acta racial bias. Conscious. Cogn. 22, 779-787

Psychol. (Amst). 152, 34-41

Peelen, M. V., and Downing, P. E. (2007). The neural basis of Riemer, M., Kleinböhl, D., Hölzl, R., and Trojan, J. (2013). visual body perception. Nat. Rev. Neurosci. 8, 636-648. doi: Action and perception in the rubber hand illusion. Exp. brain $10.1038 /$ nrn 2195

Res. 229, 383-393

Peled,A., Ritsner, M., Hirschmann, S., Geva, A. B., and Modai, Rizzolatti, G., Scandarola, C., Matelli, M., and Gentilucci, M. I. (2000). Touch feel illusion in schizophrenic patients. Biol. (1981). Afferent properties of periarcuate neurons in macaque Psychiatry 48,1105-1108 monkeys. II.Visual responses. Behav. Brain Res. 2, 147-163

Perez-Marcos, D., Sanchez-Vives, M.V., and Slater, M. (2012). Rochat, P., and Morgan, R. (1995). Spatial determinants in the Is my hand connected to my body? The impact of body continuity perception of self-produced leg movements by 3- to 5-month-old and arm alignment on the virtual hand illusion. Cogn. Neurodyn. infants. Dev. Psychobiol. 31, 626-636

6, 295-305

Rohde, M., Di Luca, M., and Ernst, M. O. (2011). The rubber

Petkova, V. I., Björnsdotter, M., Gentile, G., Jonsson, T., Li, hand illusion: feeling of ownership and proprioceptive drift do not 
Rossetti, Y., Desmurget, M., and Prablanc, C. (1995). Vives, M. V (2008). Towards a digital body: the virtual arm Vectorial coding of movement: vision, proprioception, or both? J. illusion. Front. Hum. Neurosci. 2:6

Neuropsysiology 74, 457-463.

Slater, M., Spanlang, B., Sanchez-Vives, M. V., and Blanke, Rowland, B.A., Stanford,T. R., and Stein, B. E. (2007). A model O. (2010). First person experience of body transfer in virtual of the neural mechanisms underlying multisensory integration in reality. PLoS ONE 5:e10564

the superior colliculus. Perception 36,1431-1443

Sober, S. J., and Sabes, P. N. (2003). Multisensory integration Sacks, O. (1985). The Man Who Mistook His Wife for A Hat during motor planning. J. Neurosci. 23, 6982-6992. and Other Clinical Tales. New York, NY: Summit Books.

Sober, S. J., and Sabes, P. N. (2005). Flexible strategies for Sanchez-Vives, M. V., Spanlang, B., Frisoli, A., Bergamasco, sensory integration during motor planning. Nat. Neurosci. 8, M., and Slater, M. (2010). Virtual hand illusion induced by 490-497 visuomotor correlations. PLoS ONE 5:e10381

Spence, C., Pavani, F., Maravita, A., and Holmes, N. P. Schmuckler, M. A. (1996). Visual-proprioceptive intermodal (2004). Multisensory contributions to the 3-D representation of perception in infancy. Infant Behav. Dev. 19, 221-232

Schütz-Bosbach, S., Tausche, P., and Weiss, C. (2009).

Roughness perception during the rubber hand illusion. Brain Srinivasan, M.V., Laughlin, S. B., and Dubs,A. (1982). Predictive Cogn. 70, 136-144 coding: a fresh view of inhibition in the retina. Proc. R. Soc. Lond. B 216, 427-259

Sereno, M. I., and Huang, R.-S. (2014). Multisensory maps in parietal cortex. Curr. Opin. Neurobiol. 24, 39-46

Stratton, G. M. (1896). The spatial harmony of touch and sight. Psychol. Rev. iii, 611

Seth, A. K. (2013). Interoceptive inference, emotion, and the embodied self. Trends Cogn. Sci. 17, 565-573

Tajadura-Jiménez, A., Väljamäe, A., Toshima, I., Kimura, T., Tsakiris, M., and Kitagawa, N. (2012). Action sounds Shams, L., and Beierholm, U. R. (2010). Causal inference in recalibrate perceived tactile distance. Curr. Biol. 22, R516perception. Trends Cogn. Sci. 14, 425-432 R517

Shams, L., Ma,W. J., and Beierholm, U. (2005). Sound-induced Tastevin, J. (1937). En partant de l'experience d'Aristotle flash illusion as an optimal percept. Neuroreport 16, 1923- [Starting from Aristotle's experience]. L'Encephale 1,140-158. 1927

Todorov, E., and Jordan, M. I. (2002). Optimal feedback control Shimada, S., Fukuda, K., and Hiraki, K. (2009). Rubber hand as a theory of motor coordination. Nat. Neurosci. 5, 1226illusion under delayed visual feedback. PLoS ONE 4:e6185 1235

Shimada, S., Qi, Y., and Hiraki, K. (2010). Detection of visual Tsakiris, M. (2010). My body in the brain: a neurocognitive feedback delay in active and passive self-body movements. Exp. model of body-ownership. Neuropsychologia 48, 703-712 brain Res. 201, 359-364

Tsakiris, M., Carpenter, L., James, D., and Fotopoulou, A. Shimada, S., Suzuki, T., Yoda, N., and Hayashi, T. (2014). (2010a). Hands only illusion: multisensory integration elicits Relationship between sensitivity to visuotactile temporal sense of ownership for body parts but not for non-corporeal discrepancy and the rubber hand illusion. Neurosci. Res. 85, objects. Exp. Brain Res. 204, 343-352 33-38

Tsakiris, M., Costantini, M., and Haggard, P. (2008). The role Simion, F., Regolin, L., and Bulf, H. (2008). A predisposition for of the right temporo-parietal junction in maintaining a coherent biological motion in the newborn baby. Proc. Natl. Acad. Sci. sense of one's body. Neuropsychologia 46, 3014-3018 U.S.A. 105, 809-813 
Tsakiris, M., and Haggard, P. (2005). The rubber hand illusion van Stralen, H. E., van Zandvoort, M. J. E., Hoppenbrouwers, revisited: visuotactile integration and self-attribution. J. Exp. S. S., Vissers, L. M. G., Kappelle, L. J., and Dijkerman, H. C. Psychol. Hum. Percept. Perform. 31, 80-91

(2014). Affective touch modulates the rubber hand illusion. Cognition 131, 147-158

Tsakiris, M., Longo, M. R., and Haggard, P. (2010b). Having a body versus moving your body: neural signatures of agency and van Stralen, H. E., van Zandvoort, M. J. E., Kappelle, L. J., and body-ownership. Neuropsychologia 48, 2740-2749.

Dijkerman, H. C. (2013). The rubber hand illusion in a patient with hand disownership. Perception 42, 991-993

Tsakiris, M., Prabhu, G., and Haggard, P. (2006). Having a body versus moving your body: how agency structures body- Walsh, L. D., Moseley, G. L., Taylor, J. L., and Gandevia, S. C. ownership. Conscious. Cogn. 15, 423-432

Tsakiris, M., Schütz-Bosbach, S., and Gallagher, S. (2007). On agency and body-ownership: phenomenological and Warren, D. H. (1980). Response factors in intermodality neurocognitive reflections. Conscious. Cogn. 16, 645-660 localization under conflict conditions. Percept. Psychophys. 27, 28-32

Ursino, M., Cuppini, C., and Magosso, E. (2014). Neurocomputational approaches to modelling multisensory Wei, K., and Körding, K. (2009). Relevance of error: what drives integration in the brain: a review. Neural Networks 60,141- motor adaptation? J. Neurophysiol. 101, 655-664 165

White, R. C., Aimola Davies, A. M., Halleen, T. J., and Davies, Ursino, M., Zavaglia, M., Magosso, E., Serino, A., and di M. (2010). Tactile expectations and the perception of self-touch: Pellegrino, G. (2007). "A neural network model of multisensory an investigation using the rubber hand paradigm. Conscious. representation of peripersonal space: effect of tool use," in Cogn. 19, 505-519

Engineering in Medicine and Biology Society, 2007. EMBS

2007. 29th Annual International Conference of the IEEE Wolpert, D. M., Ghahramani, Z., and Jordan, M. I. (1995). (Lyon), 2735-2739

An internal model for sensorimotor integration. Science 269, 1880-1882

Vallar, G., and Ronchi, R. (2009). Somatoparaphrenia: a body delusion.A review of the neuropsychological literature. Exp. Brain Wozny, D. R., Beierholm,U.R., and Shams, L. (2010). Probability Res. 192, 533-551 matching as a computational strategy used in perception. PLoS Comput. Biol. 6:e1000871

van Beers, R. J., Sittig,A. C., Gon, J. J., and van Der, D. (1999). Integration of proprioceptive and visual position-information: an Yuan, Y., and Steed, A. (2010). Is the rubber hand illusion experimentally supported model. J. Neurophysiol. 81, 1355- induced by immersive virtual reality? 2010 IEEE Virtual Real. 1364.

Conf. 95-102

van Beers, R. J., van Mierlo, C. M., and Brenner, E. (2011). Zeller, D., Litvak, V., Friston, K. J., and Classen, J. (2014). Reweighting visual cues by touch. J.Vis. 11,1-16

Sensory processing and the rubber hand illusion - an evoked potential study. J. Cogn. Neurosci. 27, 573-582

van den Bos, E., and Jeannerod, M. (2002). Sense of body and sense of action both contribute to self-recognition. Cognition 85, Zopf, R., Harris, J.A., and Williams, M.A. (2011). The influence 177-187

of body-ownership cues on tactile sensitivity. Cogn. Neurosci. 2, 147-154

van der Hoort, B., Guterstam, A., and Ehrsson, H. H. (2011).

Being Barbie: the size of one's own body determines the perceived Zopf, R., Savage, G., and Williams, M. A. (2010). Crossmodal size of the world. PLoS ONE 6:e20195

congruency measures of lateral distance effects on the rubber hand illusion. Neuropsychologia 48, 713-725 



\section{CHAPTER 6}

\section{Sliding Perspectives:}

\section{DISSOCIATING OWNERSHIP FROM}

\section{SELF-LOCATION DURING FULL BODY}

\section{ILLUSIONS IN VIRTUAL REALITY}

Related publication:

Sliding perspectives: dissociating ownership from self-location during full body illusions in virtual reality. Maselli,A., and Slater, M.

Front. Hum. Neurosci. 8:693, 2014 


\section{Abstract}

Bodily illusions have been used to study bodily self-consciousness and disentangle its various components, among other the sense of ownership and self-location. Congruent multimodal correlations between the real body and a fake humanoid body can in fact trigger the illusion that the fake body is one's own and/or disrupt the unity between the perceived self-location and the position of the physical body. However, the extent to which changes in self-location entail changes in ownership is still matter of debate. Here we address this problem with the support of immersive virtual reality. Congruent visuotactile stimulation was delivered on healthy participants to trigger full body illusions from different visual perspectives, each resulting in a different degree of overlap between real and virtual body. Changes in ownership and self-location were measured with novel self-posture assessment tasks and with an adapted version of the cross-modal congruency task. We found that, despite their strong coupling, self-location and ownership can be selectively altered: self-location was affected when having a third person perspective over the virtual body, while ownership toward the virtual body was experienced only in the conditions with total or partial overlap.Thus, when the virtual body is seen in the far extra-personal space, changes in self-location were not coupled with changes in ownership. If a partial spatial overlap is present, ownership was instead typically experienced with a boosted change in the perceived self-location. We discussed results in the context of the current knowledge of the multisensory integration mechanisms contributing to self-body perception. We argue that changes in the perceived self-location are associated to the dynamical representation of peripersonal space encoded by visuotactile neurons. On the other hand, our results speak in favor of visuo-proprioceptive neuronal populations being a driving trigger in full body ownership illusions. 


\section{INTRODUCTION}

A large body of experimental research in cognitive neuroscience has exploited bodily illusions to study bodily self-consciousness. This work has established a tight link between self-consciousness and the processing of multisensory bodily signals performed in specific brain areas (Makin et al., 2008; Ehrsson, 2011; Blanke, 2012). It further allowed disentangling distinct components of bodily selfconsciousness and their candidate neural representations (Longo et al., 2008; Blanke and Metzinger, 2009; Tsakiris et al., 2010).

The present study focuses on the mutual relation between two important components of bodily self-consciousness: the feeling of owning a body (body ownership) and the experience of the body occupying a given portion of space in the environment (self-location). Although in normal conditions ownership and self-location are tightly tied, recent experimental work has shown that these two components are, at least partially, separable (Longo et al., 2008). However, so far it has not been explored whether these two components are intrinsically coupled or are rather dissociable despite their strong coupling (Serino et al., 2013). In this article we present a study performed with the support of virtual reality (VR) technology, and designed to specifically test the hypothesis that, under specific experimental conditions, ownership and self-location can be selectively altered. The rationale behind the current study is based on a series of previous studies that are reviewed in the following.

\subsection{Experimental Manipulations of the Sense of Self-Location}

Self-location has been mainly investigated through experimentally induced out-of-body experiences (OBE). The experimental paradigm to induce an OBE in healthy subject consists in participants wearing a head-mounted display (HMD) occluding the physical body and displaying the back of their own body -or that of a mannequin- as filmed from a distance of roughly two meters. The visual perspective then coincides with the location of the real body and is dissociated from the location of the virtual body seen through the HMD. The illusion is then triggered through congruent visuotactile stimulation on the real and fake body. When synchronous visuotactile stimulation is delivered on the back (Lenggenhager et al., 2007), most participants experience the illusion of looking at their body from the outside, similarly to what is reported in OBEs of neurological origin (Blanke and Mohr, 2005). This illusion is associated with illusory changes in self-location, with a systematic shift from the participant's visual perspective toward the seen body. Such a drift has been consistently found throughout a variety of assessments: the "walking task" (Lenggenhager et al., 2007) and the "mental ball dropping" task (Lenggenhager et al., 2009) provided explicit measures of the perceived self-location in external and body-centered reference frames respectively, while the crossmodal congruency task (CCT) was used to implicitly assess self-location through the localization of tactile events in the external space (Aspell et al., 2009).

Functional magnetic resonance imaging ( $\mathrm{fMRI}$ ) revealed that these illusory changes in self-location correlate with activity in the temporo-parietal junction (TPJ) and the extrastriate body area (EBA) (lonta et al., 2011). The implication of the TPJ was consistently found in lesion studies on neurological 
patients suffering aberrations in self-location (Blanke et al., 2004). Focal electrical stimulation of the TPJ was further shown to systematically trigger OBE (Blanke et al., 2002).

In a similar experimental setting, OBE illusions have been alternatively triggered by delivering synchronous visuotactile stimulation on the chest while displaying the visual stimuli at the location of the occluded physical body (Ehrsson, 2007). In contrast to what found in the "back stroking" case, participants tended to feel located at the position of the physical body; this was implicitly assessed by asking participants to rate how much they felt at the location were they saw their body or at the camera's (i.e., the physical body's) location (Guterstam and Ehrsson, 2012). The difference is probably due to the fact that, in the "chest stroking" case there is no spatial conflict between visual and tactile cues. In the "back stroking" case, it probably the spatial misalignment of visual and tactile synchronous cues that induces a recalibration in the perceived self-location.

\subsection{Experimental Manipulations of the Sense of OWnership}

The sense of ownership toward a full body has been largely studied making use of the full body ownership illusion (FBOI), defined as the continuous and consciously impermeable feeling that a virtual/fake full body, including all its body parts, belongs to us in its integrity.

Built up on the rich experimental literature on the rubber hand illusion (Botvinick and Cohen, 1998), the experimental paradigm to elicit a $\mathrm{FBOI}$ consists in outfitting participants with an immersive HMD that occludes the real body and displays in its place a fake humanoid body, which may be a filmed mannequin (Petkova and Ehrsson, 2008) or a virtual character (Slater et al., 2010). Unlike the case of OBE, participants have thus a first person perspective (1PP) of the fake body usually displayed in the same posture as the real body. This setup provides participants with congruent visuo-proprioceptive cues, i.e., visual information about the virtual body's spatial configuration are congruent with perceptual cues about the body posture delivered by muscle spindle and join receptors. In the case of a static filmed mannequin, synchronous visuotactile stimulation was found to be the necessary trigger for eliciting the illusion (Petkova and Ehrsson, 2008). However, it was shown that other modalities of multisensory/multimodal correlations could trigger the illusion with no need for synchronous visuotactile stimulation. In particular, visuomotor correlations are extremely efficient in eliciting a FBOI (Slater et al., 2010; Kokkinara and Slater, 2014), thanks to the rich information processing involved in the sensorimotor control loop. On the other hand, if the fake body has a highly realistic humanoid appearance, the illusion may be elicited by congruent visuo-proprioceptive cues alone, with no need for further multimodal correlations (Maselli and Slater, 2013).

Healthy subjects experiencing a FBOI report that the seen fake body was perceived as if it was their own body and as if perceptual experiences, e.g., tactile events, were generated from the fake body. Apart from self-reports and questionnaires, the FBOI is indeed evaluated by monitoring autonomic responses to events threatening the fake body. For example, when attacking or threatening the integrity of the fake body during a FBOI, significant increases in skin conductance response (Petkova 
and Ehrsson, 2008) and in heart rate deceleration (Slater et al., 2010; Maselli and Slater, 2013) have been found. Importantly, the measured change in autonomic responses is positively correlated with the intensity of the illusion inferred from questionnaire scores. By monitoring brain activity with fMRI during a FBOI, it was found that the illusion correlates with activity in bilateral ventral premotor cortex (vPMc), left intraparietal cortex and left putamen (Petkova et al., 2011a). Interestingly, the ventral premotor cortex activation was found to be particularly associated with the construction of the unitary experience of a full body, ensuing from the merging of the sense of ownership of different body parts. No implication of the TPJ during a FBOI has been reported.

An alternative approach to explore the sense of ownership, complementary to the FBOI, is the "chest stroking" OBE illusion described in the previous section (Ehrsson, 2007; Guterstam and Ehrsson, 2012).Autonomic responses to threat events have shown how, in this illusion, the sense of ownership is "attached" to the not visible real body located at the position of the visual perspective (Ehrsson, 2007; Guterstam and Ehrsson, 2012), while the sense of ownership toward the body seen in the extra-personal space is disrupted (Guterstam and Ehrsson, 2012).

\subsection{Do OWnership and Self-Location Have Different Neural Representations? Hints from Full Body Illusions}

When comparing results from the OBE and $\mathrm{FBO}$ experiments discussed above, several hints emerge suggesting that ownership and self-location might have different origins and neural representations (Maselli and Slater, 2013; Serino et al., 2013).

First, as discussed above, $\mathrm{PMRI}$ studies revealed that $\mathrm{OBE}$ and $\mathrm{FBOI}$ are associated with the activation of different brain areas (lonta et al., 2011; Petkova et al., 2011a). Second, the experimental paradigms used for inducing $\mathrm{OBE}$ and $\mathrm{FBOI}$ were developed to explicitly assess either changes in self-location or in ownership, and not both at the same time.

In 1PP FBOI, self-location is not altered at all as the real and virtual body are collocated. On the other hand, the OBE experiments reviewed above have been mainly designed to accurately measure changes in the perceived self-location. The concurrent feeling of ownership toward the body seen in the extra-personal space was mainly assessed through questionnaire and free reports. When pooled together, data from questionnaires and self-reports in "back stroking" OBE show that illusory changes in self-location are not systematically associated with the sense of ownership: self-reports from some participants (lonta et al., 2011) suggest that this illusion is associated with self-recognition rather than with the experience of owning the virtual body (e.g." "I had the impression of being touched by the stick as if I was between two mirrors and I could see my back"; "I had the impression of watching a photo of myself..."). So it is possible that questionnaire data also reveal an enhanced sense of self-recognition rather than ownership toward the virtual body (Petkova et al., 2011b; Maselli and Slater, 2013). This possibility is further supported by the fact that participants score higher to the question "It felt as if the virtual body/mannequin was my body," when they see their own body rather than a mannequin filmed from the back (Lenggenhager et al., 2007). This modulation with the appearance of the virtual 
body is not found instead in 1PP illusion, in which the virtual body could importantly differ from the real in terms of age, gender and race, without reducing the illusion (e.g., Slater et al., 2010; Peck et al., 2013).

Only with few studies attempted so far to assess ownership in the "back stroking" OBE paradigm through autonomic measures: widespread decrements in body temperature associated with this OBE illusion have been recently reported (Salomon et al., 2013) and, based on previous RHI analogous results (Moseley et al., 2008), interpreted as a shift of the sense of ownership from the real to the virtual body. However, these results are not conclusive, as the effects found were smaller than the thermometer's resolution. Pomés and Slater (2013) measures instead heart rate deceleration (HRD) and movements in responses to a threat to the virtual body. Although no significant modulation of the HRD was found, their multivariate analysis suggests that the sense of ownership can positively contribute to sensations of drifting toward the virtual body. Still, these results do not provide any direct evidence for modulations of ownership being systematically driven by an altered sense of self-location.

Upon direct comparison between 3PP and 1PP conditions, it has been systematically found that ownership is significantly suppressed in 3PP with respect to 1PP (Slater et al., 2010; Petkova et al., 2011b; Maselli and Slater, 2013). Results from the "chest stroking" OBE illusions further support these finding, showing how, in this full body illusion the sense of ownership toward the real body seen from a 3PP is disrupted (Guterstam and Ehrsson, 2012).

In summary, no direct experimental evidence is currently available for effective changes in selflocation driven by OBE illusions to imply systematic changes in ownership. This scenario yields the motivation for the present study. In fact, the current experimental evidence suggests that ownership and self-location may have different neural representations and that they may be -at least to some extent- dissociated from each other. In this study we explicitly tested this hypothesis and further addressed the issue of how ownership and self-location interact when both are simultaneously altered in controlled experimental setups. The study is based on a set of three independent experiments conducted with the support of immersive virtual reality.

\section{The Cross-Modal Congruency Task as a Measure of Self-Location}

An important part of the results presented in this work relies on the use of the cross-modal congruency task (CCT). In this section we give a short introduction to clarify the principles that validate the $\mathrm{CCT}$, under specific configurations, as a tool to measure self-location in external space

The CCT is a standard psychophysics test that has been designed to study interactions between vision and touch (Spence et al., 2008). The task consists of discriminating the elevation of perceived target vibrations in the presence of visual distracter stimuli (Driver and Spence, 1998b). In the 
classical configuration an array of four vibrators and four light emitting diodes are arranged on the participant's hands (two on the indexes and two on the thumbs). On each trial a vibration and a light flash are presented and the participant has to make a rapid judgment on whether the vibration was up (on the index) or down (on the thumbs), irrespectively of the side and of the location where the flashing light switched on. A large number of studies have consistently shown that vibrotactile discrimination is delayed and less accurate when the distracting visual cue is incongruent in elevation with the target vibration. The effect is quantified in terms of the cross-modal congruency effect (CCE), defined as the difference in performances (e.g., response times or error rates) between incongruent and congruent trials. Because visuotactile interactions are stronger when the visual cue is presented closer to the target tactile stimulation, i.e., on the same side/hand, CCE values are typically larger when computed for trials with visual and tactile cues on the same side with respect to the opposite side (Spence et al., 2004).

Three different processes have been proposed as responsible for the observed visuotactile interaction: cuing of exogenous spatial attention, multisensory integration (MSI), and response competition (light distracters priming the response) (Spence et al., 2004). These different mechanisms may dominate the CCE or contribute to it in combination with others, depending on the time separation, i.e., the stimulus onset asynchrony (SOA), between the target and the distracter stimulus. MSI mechanisms dominate the CCE for SOA smaller than 100 ms (irrespectively of the stimuli order), while for SOAs outside this range the effect is mainly driven by exogenous attention or priming effects (Shore et al., 2006).

Using an appropriate SOA, the CCT can be therefore used as a tool for detecting the MSI of visual and tactile stimuli. The crossmodal effect found in a variety of spatial configurations can be indeed explained on the basis of the known spatial properties of bimodal visuotactile (VT) neurons found in the human and non-human primate's brain: the effect is preserved when the hands are crossed (Driver and Spence, 1998a; Maravita et al., 2003), showing how visual distracters are processed in the corresponding hand-centered reference frame, rather than in retinotopic coordinates. An analogous modulation is found in VT neural ppulations having visual receptive fields (vRFs) that shift in space along with body movements (Graziano and Gross, 1993; Duhamel et al., 1998; Lloyd et al., 2003; Brozzoli et al., 2012). Interestingly, crossmodal interactions were found also when the visual distracters were located on tools that have been actively manipulated (Maravita et al., 2002). This provided an additional behavioral counterpart for the properties of parietalVT neurons in the macaque monkey whose vRFs extend outwards after active tool manipulation (Iriki et al., 1996).

All these results are crucial in pointing out how the CCT can be exploited to infer the location of tactile events as perceived in external space. Following this rationale, previous studies used the CCT to test the spatial recalibration of the limb position occurring during a rubber hand illusion (RHI): effective crossmodal interactions were found despite the fact that vibrators and visual distracters (located on the rubber hands) were not aligned in external space (Pavani et al., 2000; Zopf et al., 2011), but only when the illusion was experienced. These results showed a recalibration of the perceived location the tactile stimuli, hence of real hands, toward the location of the rubber hands.

Similarly, the CCT has been adopted to infer shifts in the perceived location of the whole body, i.e., 
to test self-location, during OBEs (Aspell et al., 2009), with the arrays of lights and vibrators located on the back of the virtual and physical bodies respectively. An effective crossmodal interaction was found during the experienced OBE illusion, but only adopting a SOA of $233 \mathrm{~ms}$, which falls in the regime in which crossmodal congruency effects are dominated by exogenous spatial attention rather than by MSI (Shore et al., 2006). No effect was found however for SOA range dominated by $\mathrm{MSI}$ effects, thus the interpretation of Aspell et al's results as due to illusory change in self-location is not straightforward.

\section{Materials and Methods}

\subsection{PARTICIPANTS}

A total of 51 subjects were recruited for the study. Experiment 1 involved 15 naïve participants (12 females) with average age 21.6 years ( \pm 3.7 years $S D$ ); Experiment 2,17 naïve participants (8) with average age 20.4 years ( \pm 2.3 years $S D$ ); Experiment 3,19 naïve participants (10 females) with average age 22.7 years $( \pm 5.0$ years $S D)$. Participants in all the three groups had little or no previous experience in virtual reality (scores to the question "Have you ever experienced virtual reality before?" on a Likert scale from 1 to 7 had a median of 1 and interquartile range (IQR) of 1 for all the three experiments). All participants had normal or corrected to normal vision and had no history of neurological or psychiatric disorders. Participants signed an informed consent form before taking part to the experiment, and received a compensation of $€ 10$ after completion. The experimental protocol was approved by the "Comité Ético de Investigación" of the University of Barcelona, in line with the institutional ethics and national standards for the protection of human participants. In accordance with ethical commitments, all participants were contacted by email within 3-6 weeks after the experiment, and were asked whether they had any positive or negative thoughts about their experience in the experiment. None of the participants reported negative post-experimental sensations.

\subsection{Materials}

Experiments were implemented and conducted with the support of VR technology. The virtual environment was a faithful reproduction of the laboratory in which the experiments took place, modeled in 3D Studio Max by a graphics artist (Figure 1). The environment was controlled with the Unity ${ }^{1}$ platform. Participants entered the virtual environment through a stereo NVIS nVisor SX $111^{2} \mathrm{HMD}$ (dual SXGA displays with $76^{\circ} \mathrm{H} \times 64^{\circ} \mathrm{V}$ field of view (FOV) per eye, with $50^{\circ}(66 \%)$ of overlap in the horizontal axis, resulting in a total field of view of $102^{\circ}$ horizontal and $64^{\circ}$ vertical; image resolution: $1280 \times 1024$ per eye; frame rate: $60 \mathrm{~Hz}$ ). Head tracking was performed by a six degree of freedom (DoF) Intersense IS-900 device based on a coupling of infrared monitoring and

1 http://unity3d.com/

2 http://www.nvisinc.com/index.php?id=48 
inertial technology ${ }^{3}$. The tracked data were streamed in real time using theVirtual Reality Peripheral Network ${ }^{4}$ (VRPN) protocol to the Unity project.
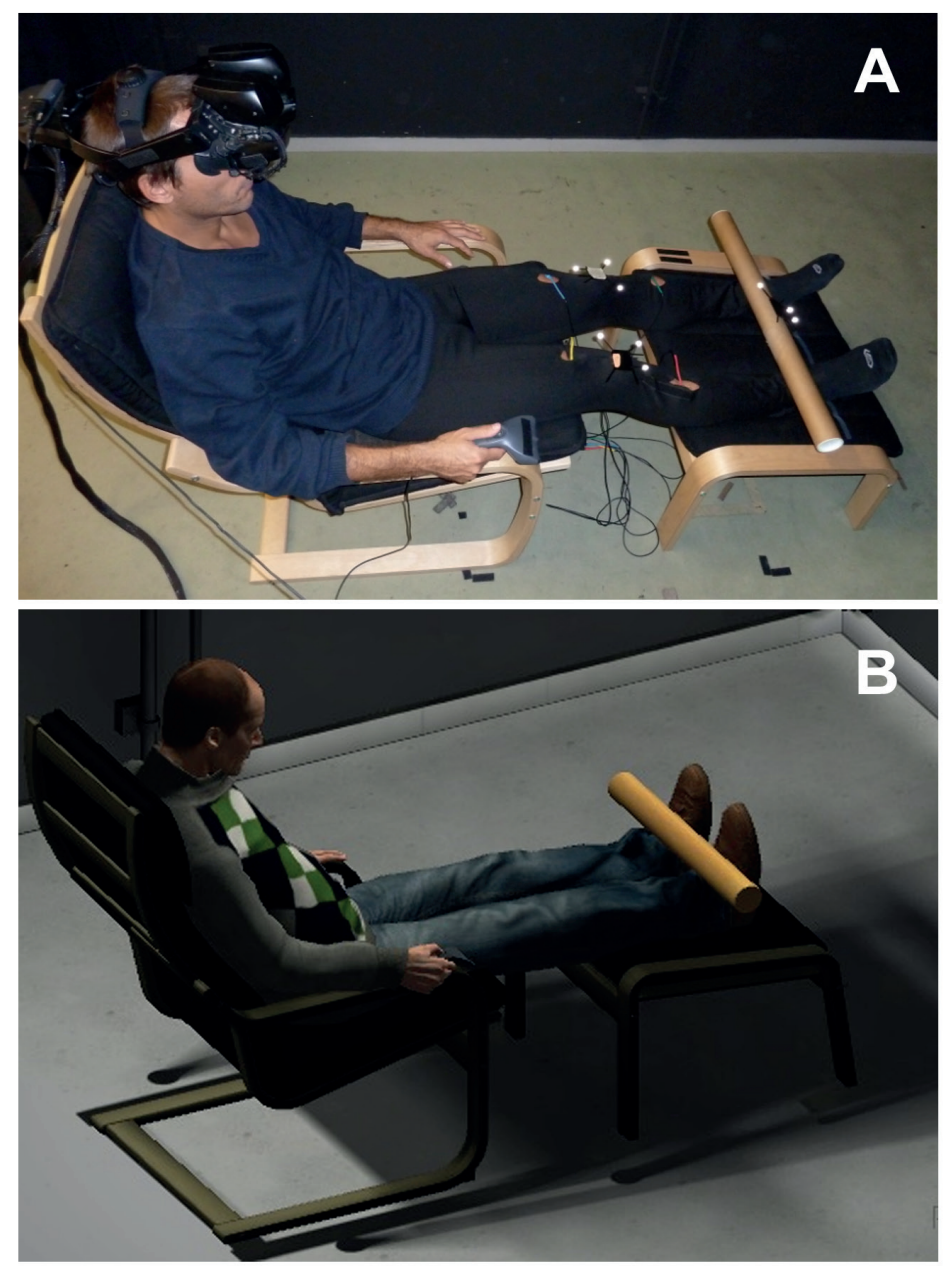

Figure 1. Experimental setup. The virtual environment (B) was a replication of the laboratory in which the experiment took place (A). Participants experienced the environment through a stereo wide field-of-view head mounted display. Four vibrators, controlled by an Arduino board, were fixed on the participant legs for the cross-modal congruency task. A cardboard tube was used to deliver continuous visuotactile stimulations symmetrically on the two legs in some of the experimental conditions.

Visuotactile stimulation was delivered on the participant legs with a tracked cardboard tube, $70 \mathrm{~cm}$ in length and $5 \mathrm{~cm}$ in diameter. The tube's movements were tracked using the Tracking Tools toolkit of Optitrack ${ }^{5}$, a system form object motion tracking that uses 12 infrared cameras. Data from a 3D optical marker attached to the physical tube were streamed in real time (via theVRPN) to the Unity

3 http://www.intersense.com/pages/20/14

4 http://www.cs.unc.edu/Research/vrpn/index.html

5 http://www.naturalpoint.com/optitrack/products/tracking-tools/ 
project and were used to control the movements of a virtual tube. Two optical markers were used to register the exact position of the knees of the participants with those of the virtual avatar, and other two were used in Experiment 3 for monitoring the position of the two hands. The length of the avatar's legs was scaled so to match the actual legs length for each participant. This allowed a precise colocation of the physical and virtual bodies in the 1PP condition, an exact spatial overlap of the vibrators arranged on the real legs and the virtual light distracters (LD), and further an accurate displacement of the virtual tube, which assured it to be displayed at exactly the same location on the real and virtual legs.

A set of four small vibrating motors ${ }^{6}$ was used for the CCT. The motors ( $3 \mathrm{~V}, 100 \mathrm{~mA}, 50 \Omega, 1200 \pm$ 300 r.p.m.) were controlled through an Arduino board connected to the Unity project. Participants used an Intersense joystick to give responses in the CCT and in the "Tube Task" described below.

\subsection{Response Variables: Measures, Data Processing and Statistical Methods}

\subsubsection{Crossmodal Congruency Test (CCT)}

The CCT was used in our experiments to test possible shifts in the perceived self-location toward the virtual body. The rational for this choice has been exposed in details in Section 2.The array of LDs/vibrators used for the CCT was arranged on the virtual/physical legs. This novel configuration had been set to detect effects of multisensory integration of tactile events on the real body and visual events on the virtual body, while having different visual perspectives of the virtual body. The four vibrators were positioned on the participant's legs using elastic bands: two on the thighs, $10 \mathrm{~cm}$ above the knees, and two on the external side of the shins (on the tibialis anterior muscle), $10 \mathrm{~cm}$ below the keens. Four virtual LDs (round red lights with $\sim 120 \mathrm{~mm}^{2}$ surface) were located in the corresponding positions on the virtual body. Each trial of the CCT consisted of a visual cue switching on during $33 \mathrm{~ms}$ and followed, after a $50 \mathrm{~ms}$ SOA, by a target vibration of $100 \mathrm{~ms}$. Due to different delays in the video stream to the HMD and in the vibrators' activation (measured in the laboratory with an oscilloscope device), the effective SOA was about $20 \mathrm{~ms}$ with the light stimuli leading vibrations. Participants were instructed to keep their gaze on a fixation point (a blue dot in the middle of the LDs array) and, for each trial, to indicate as fast and as more accurately as possible whether the delivered target vibration was above (i.e., on the thighs) or below (i.e., on the lower legs), irrespectively of the side and of the LD's location. Participants gave responses using the upper (for above) and lower (for below) buttons of a joystick held in their right hand.

Each CCT run consisted of 180 trials: 160 experimental trials, 40 for each of the four possible combinations of LD and vibration locations, plus 20 "no-go" trials. In the "no-go" trials the fixation point changed color (from blue to fuchsia) and participants were instructed to stop giving responses even if target vibrations were released. "No-go" trials were included in order to make sure that participants kept their eyes open and focused on the fixation point throughout the whole duration of the task (Zopf et al., 2010); subjects that responded to more than $30 \%$ of the no-go trials were

$6 \quad$ Flat motor, model C1034-50L, by Shenzhen Linglong Electronics Co., Ltd 
excluded from the CCT data analysis. The 180 trials were presented in a random order.

The CCT data were initially processed to extract the average response time (RT) and error percentage (EP) for each participant as a function of side (left leg vs. right leg), congruency (in elevation) and experimental condition. Trials with incorrect responses or with RT larger than 1500 ms or smaller than 200 ms, were discarded. The two measures were then combined into the inverse efficiency (IE) score, defined as the ratio between the reaction time and the percentage of correct responses [IE $=\mathrm{RT} /(1-\mathrm{EP})]$, which provides the most informative summary of the data available and compensate for possible speed-accuracy trade-offs (Townsend and Ashby, 1983; Kennett et al., 2001; Spence et al., 2001).

We chose to perform the statistical analysis on the IE data because of the better informative content of this variable with respect to the more commonly used RT (Bruyer and Brysbaert, 2011). The IE data were first analyzed in a Three-Way repeated measures ANOVA $(3 \times 2 \times 2)$ with interactions, with factors condition, side and congruency. This can detect the cross-modal interactions in terms of a main effect of congruency -because of the longer response times and the higher error rate in incongruent trials- and any interaction between congruency and side -which shows that the crossmodal interaction is stronger for stimuli close in space-. A significant interaction among the three factors would detect critical differences among the various experimental. Pairwise comparisons were then performed running a One-Way repeated measurement ANOVA on CCE-IE (the CCE calculated on the performance variable IE) with the single factor side: as discussed above, a significant modulation of the CCE by side gives hints for a perceived spatial alignment between the arrays of vibrators and LDs, and in turns provides a proxy for the perceived self-location.

\subsubsection{The "Tube TASk" (TT)}

The "Tube Task" (TT) is a novel test designed to gather measures of ownership and self-location. Participants were instructed to use a joystick to adjust the size (Experiment 1 ) the size plus the later position (Experiment 2) of a virtual tube, so to match with its extremities the perceive locations of their external ankles.

During the task no virtual body was displayed and a blue virtual tube was the unique visible object in the environment. The tube was displayed horizontally so to pass through the participant's ankles locations.At the beginning of the task the tube was centered on the middle point of the participant's ankles, had length of $114 \mathrm{~cm}$ and a fixed $5 \mathrm{~cm}$ diameter. Participants performed the task immediately after entering the virtual environment through the HMD.This measure provided the subject's baseline. The task was then repeated after each experimental condition

According to the variant used in the experiment, the task returned one or two measures: $\Delta$ TubeSize, defined as the difference between the size of the tube set after each condition and its baseline value, provided a measure of changes in the perceived posture (i.e., the legs separation relative to the midline). $\Delta$ TubeShift, defines as the difference between the position of the tube set after each condition and its baseline value, provided instead an estimate for the perceived drift of the body midline, thus for self-location. 
In the specific experimental design adopted in this study, we propose to assess ownership by looking at the effects of the strong visuo-proprioceptive coupling between the real and virtual body that is established during a FBOI (Maselli and Slater, 2013). The TT was then designed as tool for detecting such visuo-proprioceptive coupling: the experimental manipulations adopted included a change in posture of the virtual body whose legs spread apart while participants stayed still in the initial posture. Significantly positive values of $\Delta$ TubeSize will then be a proxy for an effective visuoproprioceptive coupling between the real and virtual bodies, able to affect proprioception just via visual cues, and thus a proxy for the ownership illusion. Before applying this new method for testing the experimental hypotheses under study, the validity of the TT was tested in Experiment 1.

In Experiment 1, in which participants were asked to adjust only the tube's size, TT data were analyzed using One-Way repeated measures ANOVA with the single factor condition applied on the $\Delta$ TubeSize. In Experiment 2 the same analysis was applied separately to $\Delta$ TubeSize and $\Delta$ TubeShift. Pairwise comparisons between experimental conditions were then performed running paired t-tests.

\subsubsection{The "Ankles Pointing Task” (APT)}

This task was designed -through pilot studies- as an alternative to the TT for ownership assessments. As discussed in Section Discussion of the Results, we found that, while the TT is a sensitive test for ownership (via posture assessments) when only the length of the tube is controlled, it loses such sensitivity when combining ownership and self-location assessments and giving participants the possibility to change the tube's size and concurrently its lateral position. By comparing results from Experiments 1 and 2, it was indeed clear that tube's shifting option deteriorated the sensitivity in the feet separation estimate. For this reason we introduced the APT and designed it so that it could provide veridical estimates of the legs separation in a body centered reference frame, irrespectively of the visual perspective that participants were given in the different experimental conditions.

In this case participants were asked to point with the two hands the perceived location of the external ankles. Participants performed the task in complete darkness. They were instructed to first extend both arms out and then to progressively move them in, with the palms facing each other, until each hand would point the outer ankle on the correspondent side (see Movie SOM ${ }^{7}$ ). The corresponding distance between the two hands was recorded through the tracking system (see Section Materials). As for the TT, the measure of interest was the changes in the hands' separation set after each condition with respect to its baseline value, $\triangle H S$.

As $\triangle H S$ data from the APT Experiment 3 were not normally distributed, a Wilcoxon matched pairs test was adopted to compare the $\Delta H S$ distributions in the two experimental conditions.

\subsubsection{Questionnaire}

A 12-items questionnaire (reported in Table 1) was used in Experiments 2 and 3 to assess the subjective level and quality of the illusory experience. Ownership toward the virtual body was assessed explicitly (my-body) and implicitly through responses related to the legs spreading event

7 The movie is available at https://www.frontiersin.org/articles/10.3389/fnhum.2014.00693/full when clicking on the "Open Supplemental Data" link on the right side 
(legs- $x$ ) as well as via the clothing statement that has been previously shown to positively correlate with the explicit subjective report of ownership illusion (Slater et al., 2010; Maselli and Slater, 2013). A further item was included to contrast the sense of ownership toward the virtual body with the sense of looking at another person (s-else). Changes in self-location were assessed with explicit items related to drifting sensations. Two items were included to validate the delivery of synchronous visuotactile stimulation. Each questionnaire item was scored at the end of each experimental block on a Likert scale from 1 (not at all) to 7 (completely agree) to express the level of agreement. For each item, scores given for different experimental conditions were compared pairwise via the nonparametric Wilcoxon matched pairs test.

\begin{tabular}{|c|c|c|}
\hline Item statement & Item tag & $\begin{array}{l}\text { Illusory } \\
\text { component }\end{array}$ \\
\hline $\begin{array}{l}\text { I felt that the body I saw was my own } \\
\text { body }\end{array}$ & $m y-b o d y$ & Ownership \\
\hline $\begin{array}{l}\text { I felt that I was wearing different } \\
\text { clothing than when I came to the } \\
\text { laboratory }\end{array}$ & clothing & \\
\hline $\begin{array}{l}\text { I felt as if the body I saw belonged to } \\
\text { someone else }\end{array}$ & s-else & \\
\hline I felt as if I had two bodies & two-bodies $(C)$ & \\
\hline $\begin{array}{l}\text { During the experiment, I felt as if my } \\
\text { real body was drifting to the left }\end{array}$ & drift-left & Self-location \\
\hline $\begin{array}{l}\text { During the experiment, I felt as if my } \\
\text { real body was drifting to the right }\end{array}$ & drift-right & \\
\hline $\begin{array}{l}\text { It seemed as if I was at two places at } \\
\text { the same time }\end{array}$ & two-places (C) & \\
\hline $\begin{array}{l}\text { When I saw the virtual legs } \\
\text { separating I felt as though my real } \\
\text { legs were moving }\end{array}$ & legs-moving & $\begin{array}{l}\text { Response to } \\
\text { legs separation }\end{array}$ \\
\hline $\begin{array}{l}\text { I felt a weird sensation in my legs } \\
\text { when I saw the virtual legs } \\
\text { separating }\end{array}$ & legs-weired & \\
\hline $\begin{array}{l}\text { When I saw the virtual legs spreading } \\
\text { apart, I felt as the instinct to spread } \\
\text { apart my real legs }\end{array}$ & legs-instict & \\
\hline $\begin{array}{l}\text { It seemed as though the touch I felt } \\
\text { was caused by the carton tube that I } \\
\text { was seeing moving on the virtual } \\
\text { body }\end{array}$ & touch-tube & $\begin{array}{l}\text { Visuo-tactile } \\
\text { implementation }\end{array}$ \\
\hline $\begin{array}{l}\text { It seemed as tough I felt the touch in } \\
\text { the location where the carton tube } \\
\text { touched the virtual legs }\end{array}$ & touch-loc & \\
\hline
\end{tabular}

Table 1. Questionnaire.

Each questionnaire itemwas scored on a Likert scale from 1 (not at all) to 7 (completely agree) to express the level of areement. The (C) label denotes control items 

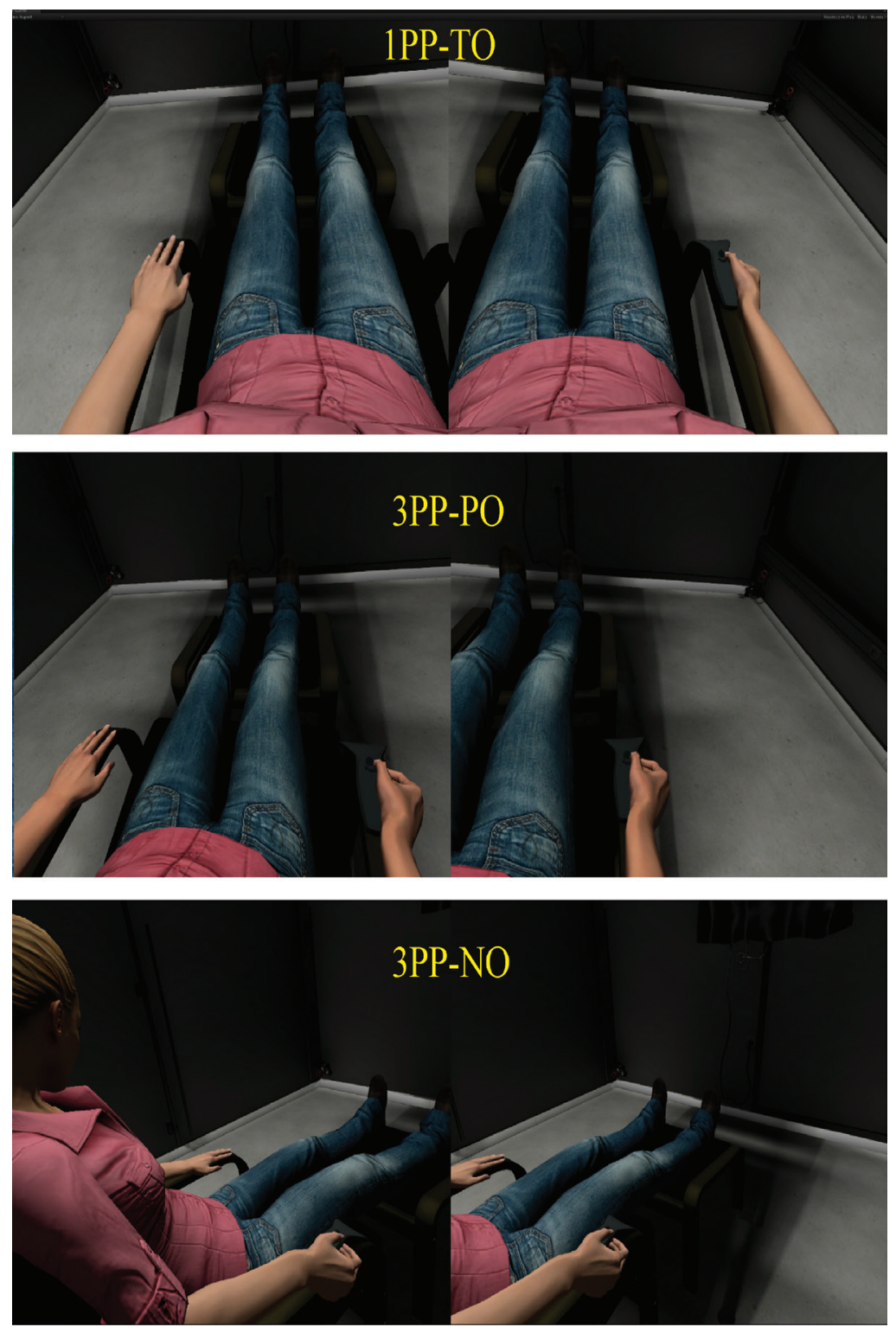

Figure 2. Different visual perspectives. According to the experimental conditions, participants had three different visual perspectives on the virtual body: a first person perspective with total overlap of the real and virtual bodies (1PP-TO); a third person perspective with partial overlap between the two bodies (3PP-PO); a third person perspective with no overlap between the two bodies (3PP-NO). In the Body and Body+VT conditions of Experiment 1, the visual perspective was the same as in 1PP-TO. 


\subsection{Experiments: Designs and Procedures}

For the three experiments a within-subjects design was adopted. Each experimental condition was presented within one experimental block. Participants went through the different conditions in a counterbalanced order across participants, so that each condition was presented in the first experimental block the same number of times.

Participants wore a pair of comfortable trousers prepared so to easily arrange the four CCT vibrators held in direct contact with the skin by elastic bands. They sit on an armchair with their foot resting on a footstool and their hands on armrests, holding a joystick with their right hand (Figure 1). The separation between the feet was adjusted by the experiments at $18 \mathrm{~cm}$. Participants were instructed to not make any movement, apart from head movements, for the whole duration of each experimental block. Before starting, the experimenter provided full instructions on how to perform the CCT and the TT or APT.All experiments started with an initial training session, preceding the experimental blocks: after a brief (about $10 \mathrm{~s}$ ) exposure to the virtual environment, with a gendermatched virtual avatar displayed from a 1PP, participants were asked to perform the TT or APT; this initial measure was stored and used as the participant's baseline. Immediately afterwards, the virtual body appeared again and a CCT training session of 30 trials was initiated.

\subsubsection{EXPERIMENT 1}

Three experimental conditions were included: in the noBody condition the virtual body was not displayed and participants saw an empty armchair when looking down. In the Body condition the virtual body was displayed to be spatially coincident with the physical body, i.e., from a first person perspective with total overlap between the real and virtual bodies (as in the upper panel of Figure 2). The Body + VT condition was the same as the Body one, but with the addition of continuous synchronous visuotactile stimulation via the tracked tube. In the noBody condition the visual perspective was the same as the first person perspective given in the two Body conditions, just the virtual body was not visible.

After completing the training phase, participants went through the three experimental blocks, each having an average duration of $10 \mathrm{~min}$. In the initial phase ( 1 $\mathrm{min})$ participants were asked to first explore the virtual scene moving the head around and then to focus on the virtual body (Body conditions) or on the chair (noBody). Only in the Body +VT, synchronous VT stimulation was delivered: the experimenter slowly moved the tracked tube on both legs, simultaneously and symmetrically, from the feet to the thighs (see Movie in the SOM ${ }^{8}$ ). The CCT was then started ( $\left.8 \mathrm{~min}\right)$. In the Body $+V T$ condition, during the CCT the tube's stroking continued symmetrical and simultaneous on both legs but was restricted to the lower part of the calf and the feet (i.e., outside the region delimited by the CCT array). At CCT completion participants were again instructed to observe the scene and focus their attention on the virtual body or the chair ( $\sim 1 \mathrm{~min})$, as in the initial phase. In the two Body conditions, the avatar's legs moved apart after about $1 \mathrm{~min}$, with the virtual feet reaching a distance of $55 \mathrm{~cm}$ in $3 \mathrm{~s}$; in the noBody condition participants just looked at the empty chair for the same period of time. The "Tube Task" was then performed and terminated the experimental block: as the visual perspective was not manipulated, participants were instructed and only had the possibility to

8 The movie is available at https://www.frontiersin.org/articles/10.3389/fnhum.2014.00693/full when clicking on the "Open Supplemental Data" link on the right side 
control the length of the tube, not its position. The HMD was removed and participants rested from 5 to 10 min before starting the next block.

The experiment was designed to test the response variables to be adopted in Experiments 2 and 3. The three main objectives were: (1) to show that the novel version of the CCT with vibrators and visual distracters arranged on the legs is equivalent to classical version (on the hands) and thus a valid tool for self-location assessments (see Section The Cross-Modal Congruency Task as a Measure of Self-Location); (2) to show that this novel version of CCT is still sensitive for detecting visuotactile integration processes on the self-body even when irrelevant visuotactile stimulation is concurrently delivered; (3) to validate the TT as a novel objective proxy for a full body ownership illusion.

In order to validate the first two points CCT's performances should reveal strong visuotactile interactions - i.e., a CCE significantly larger on the same side, with respect to opposite side- in both Body conditions. Note that we needed to validate point (2) in order to apply the CCT in the 3PP perspectives conditions of Experiments 2 and 3: in these conditions, in fact, it is necessary to keep providing visuotactile stimulation to sustain the full body illusion during the CCT, which is too long for a previously induced illusion to be sustained otherwise

In the two Body conditions in fact, participants have a 1PP over a highly collocated realistic virtual body, a condition that has been shown to be sufficient for experiencing a high sense of ownership toward the virtual body (Maselli and Slater, 2013). We then expected that the TT would be able to corroborate the sense of ownership by revealing an increase in the perceived separation between the legs at the end of both Body condition with respect to the baseline value: because participants remained in static posture throughout each experimental block, such an increase would be driven by the just seeing the virtual legs spreading apart and indeed would reveal the strong visuoproprioceptive coupling between the real and the virtual body that is established during ownership illusions. In summary, in order to validate point (3), the distance between the feet estimated through the TT should be larger than the baseline value in both Body conditions and should not vary in the control noBody condition, in which no visual cues about body posture was available.

\subsubsection{EXPERIMENT 2}

Experiment 2 was designed to test the experimental hypotheses proposed in the introduction. The specific aim of the experiment was to show that it is possible to experience illusory changes in self-location, as in OBEs, with little if any illusion of ownership over the seen virtual body.At the same time, we expected that if ownership is induced over a dislocated body the perceived self-location should be strongly affected.

As in Experiment 1, we adopted a within-subjects design with three experimental conditions, in all of which the real and virtual bodies had the same posture. In 1PP-TO condition, participants saw the virtual body from a 1PP with a total overlap (TO) between the real and virtual bodies. In the $3 P P-P O$ condition the visual perspective was shifted laterally with respect to the virtual body, while keeping a partial overlap (PO) between the two; the lateral shift was set ad-hoc for each participant 
so that the right leg of the avatar coincided in external space with the left leg of the participant (average later shift of $25 \mathrm{~cm}$ ). In the third condition, the visual perspective was shifted laterally 80 $\mathrm{cm}$ to the right of the virtual body, with no overlap (NO). The three different visual perspectives over the virtual body are shown in Figure 2. In all the three conditions synchronous visuotactile stimulation was delivered symmetrically on both legs via the tracked tube, in exactly the same way as in Experiment 1. Note to the Body + VT condition of Experiment 1 and the 1PP-TO condition of Experiment 2 were exactly the same.

The procedure was the same as that described for Experiment 1. Participants went through all experimental blocks (one for each condition) after completing the training phase. Each block had an average duration of $10 \mathrm{~min}$ : the initial phase of tube's visuotactile stimulation ( $1 \mathrm{~min})$ was followed by the CCT ( $~ 8 \mathrm{~min})$, a second phase of VT stimulation only ( $\sim \mathrm{min})$, the virtual legs spreading event (few seconds) and finally the TT. At the end of each block participants gave their scores to all questionnaire items while resting on the armchair.

The aim of the experiment was to test how ownership and self-location are modulated in FBI by shifting the visual perspective progressively away from the virtual body. Because we were interested in eliciting full body illusion we adopted the legs stroking as a trigger for the bodily illusions. The choice of the legs instead of, e.g., the chest, relays on several reasons: (i) first the legs and feet are visually prominent when looking down at our own body (if hands gesture is limited), particularly when laying in a resting posture; (ii) looking down at the chest requires a more targeted intention and is particularly uncomfortable in VR due to the HMD weight; (ii) stroking the legs allowed the stimulation of a wide portion of the whole body -from the feet to the thighs- and during the CCT to perform the stimulation while avoiding the region delimited by the CCT arrays; (iv) finally the legs separation could be easily manipulated without implying major changes in the overall resting posture.

\subsubsection{EXPERIMENT 3}

Experiment 3 was planned and designed as a follow up of Experiment 2, because of the failure of the TT in assessing changes self-posture when combined with self-location assessments (see discussion in Section The "Ankles Pointing Task" (APT) and Discussion of the Results). A within-subject design was adopted with two conditions: the 1PP-TO and the 3PP-NO of Experiment 2. The procedure was exactly the same of Experiment 2 with two differences. First the APT substituted the TT. Second, before starting each experimental block, participants were outfitted with headphones streaming white noise. This latter modification was introduced to avoid the potential inhibitory effect that conflicting visuo-auditory cues may have in the $3 P P$ condition, in which a spatial mismatch was present between the sound produced by the real tube stroking the participant's legs and the virtual tube's location on the virtual body. 


\section{Results}

The analysis reported in this Section was performed excluding outliers from the distributions under test; outliers were identified via the schematic boxplot introduced by Tukey (1977). The degrees of freedom reported for each test give information about the number of outliers excluded. Requirements for normality were always checked with the Shapiro-Wilk test (Shapiro and Wilk, $1965)$ and reported with its $p$-value $\left(P_{s W}\right)$. We avoided the debated practice of correcting for multiple comparisons (Perneger, 1998), and instead report effect sizes together with the true p-value for each performed test.

The average RT, EP and IE extracted from the CCT data and averaged across participants are given in Table 2 as a function of side, congruency, and condition, for all the three experiments.

\section{1 EXPERIMENT 1}

\subsubsection{CCT}

An average of $11 \%$ of responses were given for no-go trials across participants, none of which exceeded the $30 \%$. Figure $3 \mathrm{~A}$ shows the bar chart for the full body CCE-IE. In all conditions the effect is larger when the LD is on the same side with respect to opposite. Three-Way ANOVA with interactions showed a significant main effect of congruency $\left[F_{(1,13)}=90.8, p<0.0001\right.$, partial $\eta^{2}=$ $0.39]$ and condition $\left[F_{(2,13)}=8.7, p=0.0003\right.$, partial $\left.\eta^{2}=0.11\right]$, and a significant interaction term between side and congruency $\left[F\left(_{1,13)}=24.4, p<0.0001\right.\right.$, partial $\left.\eta^{2}=0.15\right]$. Normality of the residuals was borderline $\left(P_{s w}=0.053\right)$.

Pairwise comparisons of the CCE-IE between same and opposite side showed a significant difference for all conditions [noBody: $\mathrm{F}_{(1,12)}=7.7, \mathrm{p}=0.017$, partial $\eta^{2}=0.39 ;$ Body: $\mathrm{F}_{(1,12)}=48.1, \mathrm{p}=0.0001$, partial $\eta^{2}=0.80 ;$ Body $+V T: F_{(1,12)}=28.1, p=0.0002$, partial $\left.\eta^{2}=0.72\right]$. Residuals were normally distributed for all comparisons $\left(P_{s W}>0.3\right)$.

\subsubsection{TUBE TASK (TT)}

Figure $3 \mathrm{~B}$ shows the bar plot for the $\triangle$ TubeSize distributions in the three conditions. All were normally distributed $\left(P_{s w}>0.85\right)$. $\Delta$ TubeSize is clearly larger than zero in the two Body conditions, as confirmed by one-sample one-tailed t-tests [Body: $t_{(14)}=3.17, p=0.003$, Cohen's $d=0.82$; Body + $V T: t_{(14)}=2.36, p=0.017$, Cohen's $\left.d=0.61\right]$. This indicates a recalibration of the perceived posture. This was not the case for the noBody (control) condition. This result shows that, when the perceived posture was not affected by visual cues from the virtual body, participants reliably replicated the initial baseline estimation of their legs separation. It is important to stress that, despite de withinsubjects design, the body posture's estimate did not retain aftereffects or biases from previous exposure to the conditions in which the virtual body was seen changing its posture. 


\begin{tabular}{|c|c|c|c|c|}
\hline $\begin{array}{l}\text { Target- } \\
\text { distactor } \\
\text { congruence }\end{array}$ & $\begin{array}{l}\text { Position of } \\
\text { distracters }\end{array}$ & $\begin{array}{l}\text { Reaction } \\
\text { time } \\
\text { (RT) [ms] }\end{array}$ & $\begin{array}{c}\text { Error } \\
\text { percentage } \\
\text { (EP) [\%] }\end{array}$ & $\begin{array}{c}\text { Inverse } \\
\text { efficiency } \\
\mathrm{IE}=\mathrm{RT} /(-\mathrm{EP})[\mathrm{ms}]\end{array}$ \\
\hline \multicolumn{5}{|c|}{ EXPERIMENT 1} \\
\hline \multicolumn{5}{|l|}{ NoBody } \\
\hline \multirow[t]{2}{*}{ Congruent } & same & $600(23)$ & $1.0(0.5)$ & $606(23)$ \\
\hline & different & $632(22)$ & $2.0(0.9)$ & $644(20)$ \\
\hline \multirow[t]{2}{*}{ Incongruent } & same & $690(28)$ & $7.7(1.2)$ & $750(33)$ \\
\hline & different & $680(20)$ & $3.7(1.1)$ & $707(20)$ \\
\hline \multicolumn{5}{|l|}{ Body } \\
\hline \multirow[t]{2}{*}{ Congruent } & same & $609(29)$ & $0.4(0.2)$ & $613(31)$ \\
\hline & different & $650(29)$ & $1.0(0.4)$ & $656(29)$ \\
\hline \multirow[t]{2}{*}{ Incongruent } & same & $723(32)$ & $4.8(1.3)$ & 764 (38) \\
\hline & different & $665(28)$ & $3.9(0.5)$ & $692(30)$ \\
\hline \multicolumn{5}{|l|}{ Body+VT } \\
\hline \multirow[t]{2}{*}{ Congruent } & same & $637(32)$ & $1.3(0.6)$ & $648(35)$ \\
\hline & different & $682(36)$ & $3.6(1.3)$ & $707(36)$ \\
\hline \multirow[t]{2}{*}{ Incongruent } & same & 738 (32) & $9.2(2.2)$ & $823(50)$ \\
\hline & different & $712(31)$ & $4.8(1.4)$ & $748(30)$ \\
\hline \multicolumn{5}{|c|}{ EXPERIMENT 2} \\
\hline \multicolumn{5}{|l|}{ IPP-TO } \\
\hline \multirow[t]{2}{*}{ Congruent } & same & $638(28)$ & $2.2(0.7)$ & $653(28)$ \\
\hline & different & $684(30)$ & $5.3(1.1)$ & $723(57)$ \\
\hline \multirow[t]{2}{*}{ Incongruent } & same & $752(30)$ & $15.2(3.3)$ & 910 (31) \\
\hline & different & $713(26)$ & $7.3(2.0)$ & 776 (36) \\
\hline \multicolumn{5}{|l|}{ 3PP-PO } \\
\hline \multirow[t]{2}{*}{ Congruent } & same & $647(19)$ & $2.7(1.0)$ & $665(20)$ \\
\hline & different & $694(21)$ & $4.0(1.6)$ & $726(27)$ \\
\hline \multirow[t]{2}{*}{ Incongruent } & same & $751(23)$ & $13.8(4.8)$ & 977 (147) \\
\hline & different & $702(20)$ & $7.1(2.0)$ & 765 (35) \\
\hline \multicolumn{5}{|l|}{ 3PP-NO } \\
\hline \multirow[t]{2}{*}{ Congruent } & same & $656(21)$ & $4.7(1.2)$ & $691(26)$ \\
\hline & different & $687(23)$ & $5.8(1.1)$ & $732(58)$ \\
\hline \multirow[t]{2}{*}{ Incongruent } & same & $734(23)$ & 15.7 (3.6) & $900(28)$ \\
\hline & different & $745(23)$ & $12.7(2.4)$ & $860(32)$ \\
\hline
\end{tabular}



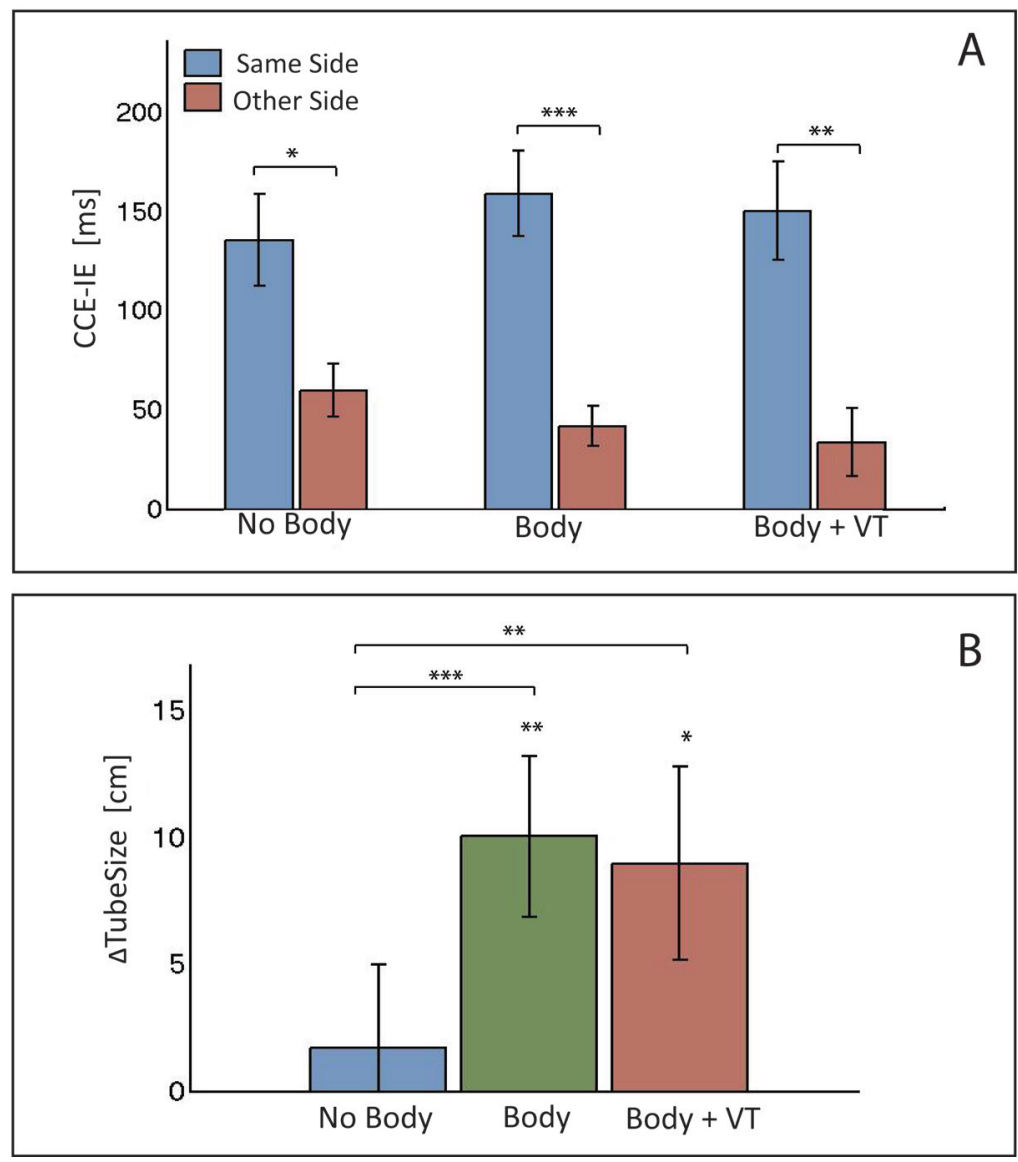

Figure 3. Results from Experiment 1. (A) Means and standard errors of the cross-modal congruency effect in inverse efficiency (CCE-IE) measured on the same side and on the opposite side, for the three experimental conditions (noBody, Body, and Body+VT). Ranges for the p-values from One-Way repeated measures ANOVA between same and other side are reported. (B) Differences (means \pm standar errors) between the tube size estimation from the "Tube Task" (TT), perfomed after and before each of the experimental conditions: noBody, Body, and Body+VT. Positive values of $\Delta T$ TubeSize indicate that participants experienced a recalibration of their perceived legs separation as a consequence of seeing the virtual legs spreading apart. Ranges for the p-values are reported for distributions with a mean significantly larger than zero (from one sample one-tailed t-test) and for paired comparisons across conditions (One-Way ANOVA). $* 0.01<p<0.05, * * 0.001<p<0.01, * * * 0.0001<p<0.001$. 
One-Way repeated ANOVA revealed a significant effect of condition on $\Delta$ TubeSize $\left[\mathrm{F}_{(2,14)}=6.5\right.$, $P=0.005$, partial $\left.\eta^{2}=0.26\right]$, with the residual being normally distributed $\left(p_{s w}=0.24\right)$. Pairwise comparisons revealed that $\triangle$ TubeSize was significantly larger for both Body conditions with respect to the noBody condition [Body vs. noBody: $t^{(14)}=3.4, p=0.002$, Cohen's $d=0.67 ;$ Body $+V T$ vs. noBody: $t_{(14)}=2.37, p=0.016$, Cohen's $\left.d=0.53\right]$. No difference between the two Body conditions was found $\left[\mathrm{t}_{(14)}=0.56, \mathrm{p}=0.58\right]$.

\subsection{EXPERIMENT 2}

One subject was excluded from the analysis because he did not understand correctly the experiment instructions and another because the vibrators array stopped working during one of the conditions.

\subsubsection{CCT}

An average of $11.8 \%$ of responses were given for no-go trials across participants, none of which exceeded the $30 \%$. Three-Way ANOVA with interactions revealed a significant main effect of congruency $\left[\mathrm{F}_{(1,12)}=68.4, \mathrm{p}<0.0001\right.$, partial $\left.\eta^{2}=0.34\right]$, a significant interaction term between side and congruency $\left[F_{(1,12)}=20.0, p<0.0001\right.$, partial $\left.\eta^{2}=0.13\right]$, and a trend for interaction of side, congruency and condition $\left[F_{(1,12)}=2.7, p=0.071\right.$, partial $\left.\eta^{2}=0.04\right]$. The requirement for normality of the residuals was satisfied $\left(P_{s W}=0.08\right)$.

Pairwise comparisons (Figure $4 \mathrm{~A}$ ) revealed a significantly higher CCE-IE on the same side with respect to opposite in 1PP-TO $\left[F_{(1,12)}=27.7, P=0.0002\right.$, partial $\left.\eta^{2}=0.70\right]$ and $3 P P-P O\left[F_{(1,12)}=74.2\right.$, $P<0.0001$, partial $\left.\eta^{2}=0.86\right]$, but not in 3PP-NO $\left[F_{(1,12)}=1.13, p=0.31\right]$. Residuals were normally distributed for all comparisons $\left(P_{S W}>0.26\right)$. Because we expected a significant difference between the CCE on same and other side also in 3PP-NO, we repeated the test with the CCE computed for the RT data (CCE-RT). In this case we found CCE-RT to be higher in the same than in the other side $\left[F_{(1,14)}=4.57, P=0.0507, \eta^{2}=0.051, P_{S W}=0.17\right]$, in line with our expectations.

It was interesting to specifically inspect performance on the 3PP-PO trials with the target vibration on the left leg of the participant. In fact, for these trials, the light distracters on the "opposite side" were located, in external space, in exactly the same positions of the vibrators (the virtual right leg overlapped in external space with the participant's left leg), while those on the "same side" were spatially incongruent. Despite this, the average CCE-IE was much higher on the same side than on the opposite (see inset in Figure 4A). A significant difference between same and opposite side was detected in this subsample with a matched-paired Wilcoxon test $\left(z_{13}=3.1, P_{13}=0.002, P S_{\text {dep }}=0.92\right)$, as when running an One-Way ANOVA the resulting residuals were not normally distributed.

\subsubsection{TUBE TASK}

Due to technical recording problems, the TT data were missing from three subjects of the 15 subjects under analysis. The Shapiro Wilk test revealed normal distributions for both $\triangle$ TubeSize and $\triangle$ TubeShift $\left(\mathrm{P}_{\mathrm{SW}}>0.24\right)$. Two outliers (one in 1PP-TO and one in 3PP-NO) were found and excluded from the analysis. 

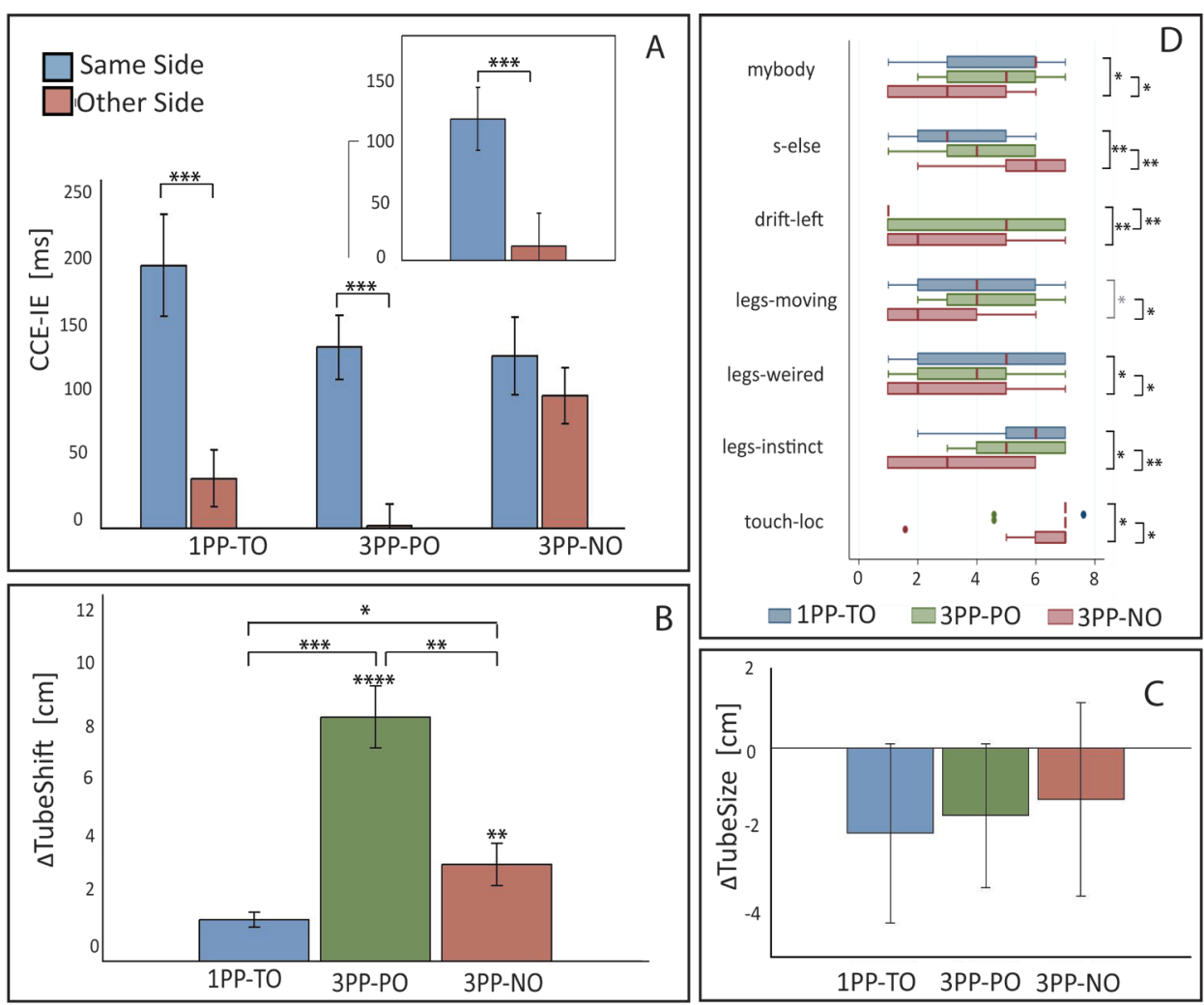

Figure 4. Results from Experiment 2. (A) Means and standard errors of the cross-modal congruency effect in inverse efficiency (CCE-IE) measured on the same side and on the opposite side, for the three experimental conditions (1PP-TO, 3PP-PO and 3PP-NO). The inset shows the same for the 3PP-PO subset of trials in which the target vibration was on the real left leg that overlapped in external space with the right virtual leg. Ranges for the $p$-values from One-Way repeated measures ANOVA between same and other side are reported. (B) Differences (means \pm standar errors) between the tube lateral position set in the Tube Task (TT) after and before each experimental condition. Positive values of $\Delta$ TubeShift indicate that participants experienced a recalibration of their self-location to the left, which is toward the virtual body. Ranges for the $p$-values are reported for distributions with a mean significantly larger than zero (from one sample t-test) and for paired comparisons across conditions (One-Way ANOVA). (C) Differences (means \pm standar errors) between the tube size estimation from the Tube Task (TT), perfomed after and before each experimental condition. The $\Delta$ TubeSize distributions match the zero mean distribution in all the three conditions. (D) Box plots showing the comparison of questionnaire data across conditions: only items for which significant differences among conditions have been found are shown. Ranges for the $p$-values from matched pairs Wilcoxon tests are reported. $* 0.01<p<0.05$, **0.001<p<0.01, ***0.0001<p<0.001, ****0<p $<0.0001$. 
Figure $4 \mathrm{~B}$ shows the bar chart for the $\Delta$ TubeShift distributions. For all conditions the distributions were normal $\left(P_{s w}>0.20\right)$. One-sample one-tailed t-test analysis revealed a distribution mean significantly larger than zero for both the 3PP-PO $\left[\mathrm{t}_{(11)}=7.59, \mathrm{P}<\right.$ 0.0001 , Cohen's $d=2.19]$ and 3PP-NO $\left[\mathrm{t}_{(10)}=2.8, \mathrm{p}=0.0097, \mathrm{~d}=0.84\right]$, but not for 1PPTO $\left[\mathrm{t}_{(10)}=1.5, \mathrm{p}=0.086, \mathrm{~d}=0.44\right]$.

One-Way repeated ANOVA revealed a significant effect of condition on $\Delta$ TubeShift $\left[F_{(2,9)}=13.66, p=0.0002\right.$, partial $\left.\eta^{2}=0.60\right]$, with normally distributed residuals $\left(p_{s W}=\right.$ $0.99)$. Pairwise comparisons revealed that $\triangle$ TubeShift was significantly larger in both 3 PP conditions with respect to 1PP-TO [3PP-PO vs. 1 PP-TO: $\mathrm{t}_{(10)}=5.4, \mathrm{P}=0.0002$, Cohen's $\mathrm{d}=2.62 ; 3 P P-N O$ vs. $\left.1 P P-T O: \mathrm{t}_{(9)}=2.2, \mathrm{p}=0.029, \mathrm{~d}=0.92\right]$. Interestingly, $\Delta$ TubeShift in the $3 P P-P O$ condition was found to be significantly larger than in $3 P P-N O\left[t_{(10)}=3.4, P\right.$ $=0.0036$, Cohen's $d=1.79]$.

The $\Delta$ TubeSize values were normally distributed in all conditions $\left(\mathrm{P}_{\mathrm{sw}}>0.24\right)$. Contrary to our expectations the TT did not detect significant changes in the perceived posture for any of the conditions (see Figure 4C). One-sample t-test performed on the three distributions revealed indeed no difference from the zero-mean distribution $(p>0.29)$. One-Way repeated ANOVA also revealed no significant difference between conditions $\left[\mathrm{F}_{(2,11)}=1.73, \mathrm{p}=0.2\right]$.

This unexpected outcome may be due to the interaction between the tube size adjustment and the additional lateral shift. In fact the 1PP-TO condition in this experiment is exactly the same as the Body +VT condition in Experiment 1, with the only difference being instructions given for the TT.The fact that in Experiment 1 the distribution of $\Delta$ TubeSize has a mean significantly larger than zero, while in Experiment 2 its mean is statistically equal to zero suggests that having the possibility to shift the tube laterally, and actually doing it, has a detrimental effect on the TT's measure of perceived body posture. The only difference between the two groups was in fact that in the latter participants had the possibility to shift the tube's laterally a part from adjusting its size.

\subsubsection{Questionnaire}

Median values and interquartile ranges are reported in Table 3 for all items. The results from matched pairs Wilcoxon tests are given in Table 4 (items for which none of the three comparisons was significant were omitted). Effect sizes are given in terms of the probability of superiority of dependent measures, PSdep, defined as the probability that the score from the condition that most frequently has the higher score will be greater than the score from the condition that most frequently has the lower score (Grisand Kim, 2012). The table highlights all significant comparisons as shaded cells. Boxplot corresponding to the significant comparisons are shown in Figure 4D. 
Table 3 | Experiment 2: Medians and interquartile ranges of the questionnaire scores.

\begin{tabular}{|c|c|c|c|c|c|c|}
\hline \multirow[b]{2}{*}{ Item } & \multicolumn{2}{|c|}{ 1PP-TO } & \multicolumn{2}{|c|}{ 3PP-PO } & \multicolumn{2}{|c|}{ 3PP-NO } \\
\hline & Median & IQR & Median & IQR & Median & IQR \\
\hline$m y$-body & 6 & 3 & 5 & 3 & 3 & 4 \\
\hline clothing & 6 & 4 & 5 & 5 & 4 & 3 \\
\hline s-else & 3 & 3 & 4 & 3 & 6 & 2 \\
\hline two-bodies & 2 & 2 & 3 & 4 & 4 & 4 \\
\hline drift-left & 1 & 0 & 5 & 6 & 2 & 4 \\
\hline drift-right & 1 & 0 & 1 & 4 & 1 & 1 \\
\hline two-places & 3 & 2 & 3 & 4 & 3 & 4 \\
\hline legs-moving & 4 & 4 & 4 & 3 & 2 & 3 \\
\hline legs-weird & 5 & 5 & 4 & 3 & 2 & 4 \\
\hline legs-instinct & 6 & 2 & 5 & 3 & 3 & 5 \\
\hline touch-tube & 7 & 1 & 7 & 1 & 7 & 1 \\
\hline touch-loc & 7 & 0 & 7 & 0 & 7 & 1 \\
\hline
\end{tabular}

\subsection{Experiment 3}

\subsubsection{CCT}

Three-Way ANOVA with interactions revealed a significant main effect of congruency $\left[\mathrm{F}_{(1,13)}=\right.$ 94.4.0, $p<0.0001$, partial $\eta^{2}=0.51$ ] and a significant interaction between side and congruency $\left[\mathrm{F}_{1,13)}=6.3, \mathrm{p}=0.014\right.$, partial $\left.\eta^{2}=0.06\right]$. Importantly, the three-way interaction between congruency, side and condition was not significant, indicating that visuotactile interactions were comparable in both conditions. The requirement for normality of the residuals was satisfied $\left(P_{s W}=0.97\right)$. Pairwise comparisons between CCE-IE on same side and opposite side (shown in Figure 5A) revealed a significant difference in both the 1PP-TO $\left[\mathrm{F}_{(1,15)}=15.7, \mathrm{P}=0.0012\right.$, partial $\left.\eta^{2}=0.51\right]$ and the 3PP-NO $\left[F(1,15)=5.79, p=0.029\right.$, partial $\left.\eta^{2}=0.28\right]$. Residuals were normally distributed $\left(p_{s w}>0.19\right)$.

\subsubsection{Ankles Pointing Task (APT)}

Data from two subjects were missing due to recording problems. Another two data sets were discharged because of the participant having misinterpreted the task instructions. A total of 15 subjects were included in the analysis. It is notable that the $\triangle H S$ estimates from these two subjects were "extreme" outliers (Tukey, 1977) in the $\triangle H S$ distribution from 3PP-NO.

The $\triangle H S$ distribution extracted from the APT was not normal, but resembled a bimodal distribution 
with one cluster around zero and one another at positive values. This was particularly the case for the 1PP-TO distribution. The cluster around zero was probably a biased from participants that based their measure on the knowledge that their feet did not mov, as explicitly reported by one of the two excluded participants.

A matched-pairs Wilcoxon rank test revealed a significant difference between the two experimental conditions $\left(\mathrm{z}_{14}=2.42, \mathrm{P}_{14}=0.016, \mathrm{PS}_{\text {dep }}=0.71\right)$, indicating that participants perceived a significantly larger distance between their own ankles in the 1PP-TO than in the 3PP-NO. The box plot of the two distributions is shown in Figure 5B.

Table 4 | Experiment 2: Paired comparisons of the questionnaire scores. 1PP-TO

\begin{tabular}{|c|c|c|c|c|c|c|}
\hline \multirow[b]{2}{*}{ Item } & \multicolumn{3}{|c|}{ 1PP-TO vs. 3PP-PO } & \multicolumn{3}{|c|}{ 1PP-TO vs. 3PP-NO } \\
\hline & $z$ & $p$ & $\mathrm{PS}_{\text {dep }}$ & $z$ & $p$ & $\mathrm{PS}_{\text {dep }}$ \\
\hline$m y-b o d y$ & 0.52 & 0.60 & & 2.05 & 0.040 & 0.73 \\
\hline s-else & 0.82 & 0.42 & & -2.71 & 0.007 & 0.87 \\
\hline drift-left & -2.98 & 0.003 & 0.8 & -2.63 & 0.009 & 0.73 \\
\hline legs-moving & 0.12 & 0.91 & & 1.94 & 0.052 & 0.70 \\
\hline legs-weired & 0.58 & 0.56 & & 2.29 & 0.023 & 0.73 \\
\hline legs-instict & 0.44 & 0.66 & & 2.28 & 0.022 & 0.73 \\
\hline touch-loc & 0.44 & 0.66 & & 2.23 & 0.026 & 0.67 \\
\hline
\end{tabular}

3PP-PO vs. 3PP-NO

\begin{tabular}{lrll}
\cline { 3 - 4 } Item & $\boldsymbol{z}$ & $\boldsymbol{p}$ & $\mathbf{P S}_{\text {dep }}$ \\
\hline my-body & 2.03 & 0.02 & 0.80 \\
s-else & -2.63 & 0.009 & 0.87 \\
drift-left & 1.66 & 0.097 & \\
legs-moving & 2.55 & 0.011 & 0.80 \\
legs-weired & 2.08 & 0.037 & 0.70 \\
legs-instict & 2.68 & 0.007 & 0.80 \\
touch-loc & 2.23 & 0.026 & 0.67 \\
\hline
\end{tabular}

Reported p-values are from matched pairs Wilcoxon tests; effect size is reported in terms of the probability of superiority of dependent measures (PSdep). Only results from comparisons revealing a significant difference across conditions are reported. Shaded cells highlight comparisons that revealed a significant difference across conditions. 

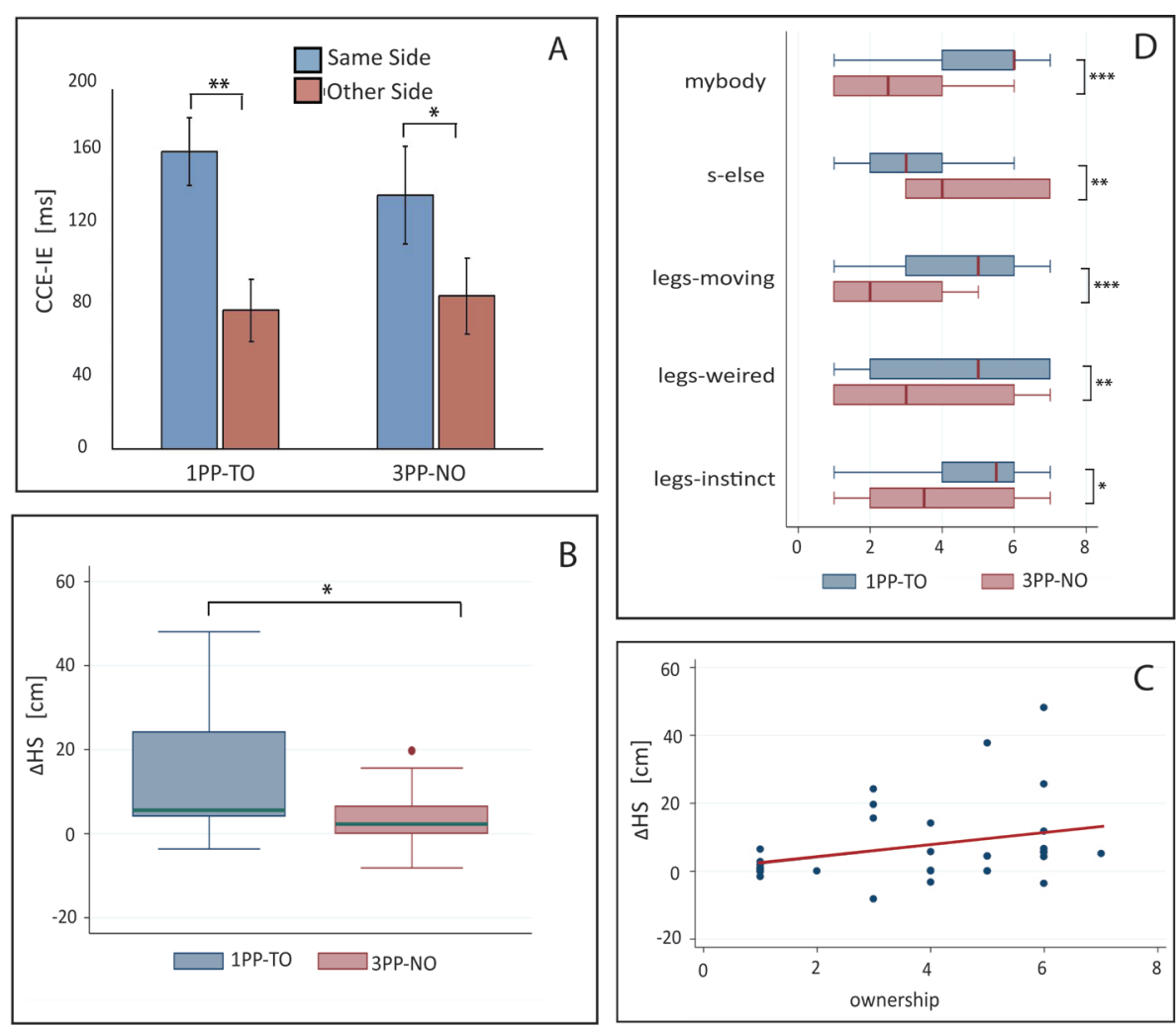

Figure 5. Results from Experiment 3. (A) Means and standard errors of the cross-modal congruency effect in inverse efficiency (CCE-IE) measured on the same side and on the opposite side, for the two experimental conditions (1PP-TO and 3PP-NO). Ranges for the p-values from One-Way repeated measures ANOVA between same and other side are reported. (B) Box plot of the differences between the hands separation measured in the "Ankles Pointing Task," after and before each experimental condition. Positive values of $\triangle H S$ indicate that participants experienced a recalibration of their perceived legs separation as a consequence of solely seeing the virtual legs spreading apart. $\triangle H S$ values were significantly larger in 1PP-TO than in 3PP-NO, showing that a proprioceptive recalibration occurred in the first condition but not in the latter. The p-value from matched pairs Wilcoxon test is reported. (C) $\Delta H S$ values are plotted against the scores to the mybody questionnaire item. A strong statistical trend $(p=0.055)$ for a positive correlation between the two variables was found. (D) Box plots showing the comparison of questionnaire data across conditions: only items for which significant differences among conditions have been found are shown. Ranges for the $p$-values from matched pairs Wilcoxon tests are reported. $* 0.01<p<0.05, * * 0.001<p<0.01$, $* * * 0.0001<p<0.001$. 


\subsubsection{QUESTIONNAIRE}

Questionnaire results are summarized in Table 5 and shown in Figure 5D. The results are consistent with those from Experiment 2, apart from the drift-left item. Scores were in fact significantly higher in 1PP-TO than in 3PP-NO for the items my-body $\left(z=3.27, p=0.001, \mathrm{PS}_{\text {dep }}=0.89\right)$ and the three items assessing the response to the legs separation event (legs-moving: $z=3.18, p=0.001, \mathrm{PS}_{\text {dep }}=0.86$; legs-weird: $z=2.65, p=0.008, P_{\text {dep }}=0.78$; legs-instinct: $z=2.29, p=0.022, P_{\text {dep }}=0.72$ ). On the other hand, scores s-else were significantly higher in 3PP-NO than in 1PP-TO $\left(z=2.9, \mathrm{p}=0.004, \mathrm{PS}_{\text {dep }}=0.78\right)$. Differently from Experiment 2, no significant difference was found for drift-left, although a weak trend for scores to be higher in 3PP-NO than in 1PP-TO was found $(z=1.52, p=0.13)$. This was due to the extremely low scores given to this item in the 3PP-TO condition across all participants, which was not the case in Experiment 2.

Table 5 | Experiment 3: Medians, interquartile ranges and paired comparisons of the questionnaire scores.

\begin{tabular}{|c|c|c|c|c|c|c|c|}
\hline \multirow[b]{2}{*}{ Item } & \multicolumn{2}{|c|}{ 1PP-TO } & \multicolumn{2}{|c|}{ 3PP-NO } & \multicolumn{3}{|c|}{ MP Wilcoxon test } \\
\hline & Median & IQR & Median & IQR & $z$ & $p$ & PS $_{\text {dep }}$ \\
\hline my-body & 6 & 2 & 2.5 & 3 & 3.27 & 0.001 & 0.92 \\
\hline Clothing & 4 & 3 & 3.5 & 4 & 1.02 & 0.31 & \\
\hline s-else & 3 & 2 & 4 & 4 & -2.90 & 0.004 & 0.78 \\
\hline two-bodies & 3 & 2 & 2.5 & 4 & -0.84 & 0.40 & \\
\hline drift-left & 1 & 0 & 1 & 1 & -1.52 & 0.13 & \\
\hline drift-right & 1 & 0 & 1 & 0 & 0.51 & 0.61 & \\
\hline two-places & 3 & 2 & 2.5 & 3 & 0.11 & 0.91 & \\
\hline legs-moving & 5 & 3 & 2 & 3 & 3.18 & 0.001 & 0.86 \\
\hline legs-weird & 5 & 5 & 3 & 5 & 2.65 & 0.008 & 0.78 \\
\hline legs-instinct & 5.5 & 2 & 3.5 & 4 & 2.29 & 0.022 & 0.72 \\
\hline touch-tube & 7 & 1 & 7 & 1 & -0.28 & 0.78 & \\
\hline touch-loc & 7 & 0 & 7 & 0 & -0.58 & 0.56 & \\
\hline
\end{tabular}

Reported p-values are from matched pairs Wilcoxon tests and effect size is reported in terms of the probability of superiority of dependent measures $\left(P S_{\text {dep }}\right)$. Shaded cells highlight comparisons that revealed a significant difference across conditions.

\subsubsection{Correlation analysis}

We tested the correlation between the level of ownership as assessed from questionnaire scores to the mybody item and the changes in the perceived legs separation measured with the APT ( $\triangle H S$ ). The Spearman's rho was showing a close to statistical significant positive correlation between the two measures $(\rho=0.34, p=0.055)$, also shown in the scatter plot in Figure $5 \mathrm{C}$. It is remarkable that 
despite the cognitive bias discussed above (participants knew that their feet did not move), which introduces extra variance in the APT data hindering correlation analysis, a positive correlation was found. This result further corroborates the validity of APT method as measure for ownership illusions.

\section{Discussion of the Results}

\subsection{EXPERIMENT 1}

Results from Experiment 1 validated the methods used in the study. It was shown that the new proposed version of CCT, with the LEDs/vibrators arranged on the legs, is a valid tool to detect effective integration of visuotactile (VT) bodily signals, and thus to detect changes in the perceived self-location during full body illusions (see discussion in Section Materials and Methods). The CCT revealed in fact effective visuotactile interactions in both the Body conditions, in which participants experienced a fully collocated virtual body from a 1PP, a condition shown to be sufficient for eliciting a FBOI (Maselli and Slater, 2013). Importantly, the effective SOA was $20 \mathrm{~ms}$, so that effective VT interactions can be primarily attributed to effective VT integration (Shore et al., 2006). In a similar setup, Aspell et al. (2009) had instead to increase the SOA to $233 \mathrm{~ms}$ in order to detect effective VT interactions when additional visuotactile stimulation was delivered during the CCT; however, as a drawback, the observed interaction was mainly dominated by exogenous attention rather than MSI (Shore et al., 2006). This difference may be due to the fact that, in our study, the visuotactile stimulation was delivered outside the region delimited by the CCT lights/vibrators array.

Interestingly, effective VT integration was detected also in the noBody condition. In previous studies, a similar condition was implemented, but with the array of visual distracters shifted with respect to the vibrators: in the "rubber hands absent" condition of Pavani et al. (2000) the vibrators were located on the unseen hands and the LEDs array was shifted $20 \mathrm{~cm}$ upward; similarly, in the "body not visible" condition of Aspell et al. (2009) the vibrators, located on the back while the distracters, were seen in the far extra-personal space. In both studies no significant visuotactile interaction was detected when the body was not visible. A significant CCE effect has instead been reported for an object condition in Salomon et al. (2012); however, as suggested by the authors, it is not straightforward to interpret this results because of the experimental design adopted, in which "object" trials where intermixed at a fast pace with "body" trials (Salomon et al., 2012). The fact that we did find evidence for visuotactile interaction also when the body is not visible is most probably due to the fact that LED distracters and vibrators were in spatial register. The result shows that the spatial information about the body provided solely by proprioception is sufficient to have a robust stored localization of body parts that contribute to the multisensory integration of visual and tactile information. This is consistent with the previous findings of the CCE modulation associated with various degree of visuo-proprioceptive spatial mismatch (Spence et al., 2008).

Experiment 1 further validated the TT/APT as novel tools for objective assessments of full body ownership illusions. The TT data showed that in the two Body conditions (in which ownership was 
experienced toward the virtual body) after seeing the virtual body moving the feet apart and getting into a new posture, participants estimated a larger distance between their feet even if the physical body did not move at all. This outcome relays, as expected, on the strong coupling of visual and proprioceptive cues from the virtual and real bodies that is established during an ownership illusion (see Section 4.4 in Maselli and Slater, 2013). In this respect, the validation of the TT as a tool to assess full body ownership through assessments of illusory changes in posture driven by movements of the virtual body only extends to the APT. In fact, in the latter differs from the TT only in the way in which participants indicated the perceived distance among their feet, which was design ad-hoc to provide estimates of the legs separation in a body centered reference frame. The validity of the APT as a proxy for the FBOI was further corroborated by the positive correlation found between ownership measures from questionnaire (scores to the mybody item) and the illusory change in body postures measured with the APT $(\Delta H S)$, found in Experiment 3.

\subsection{EXPERIMENTS 2 AND 3}

The outcome of Experiments 2 and 3 supports our main experimental hypotheses. We first discuss the set of results that show how ownership and self-location can be manipulated independently from each other, during FBOI and OBE illusion respectively. We do this by comparing data from the 1PP-NO and 3PP-NO conditions. We then discuss results that show how simultaneous changes in ownership and self-location can be induced, and do interact, when having a third person visual perspective of a virtual body that partially overlaps with the physical body, as in the 3PP-PO condition.

\subsubsection{FBOI AND OBE CAN OCCUR INDEPENDENTLY OF EACH OTHER}

As expected we found evidence for FBOI to occur in the 1PP-TO but not in the 3PP-NO in both questionnaire and APT data. Scores to the my-body item, as well as to all the three legs items, were significantly higher in 1PP-TO than in 3PP-NO, showing that ownership toward the virtual body was experienced consistently across participants, only in the 1PP-TO condition. The APT data from Experiment 3 corroborated this finding.A significantly larger change in the perceived feet separation was in fact found in the 1PP-TO condition with respect to 3PP-NO.This showed that a tight coupling between vision and proprioception, characteristic of ownership illusions, is established in the 1PPTO but not in the 3PP-NO condition. In both Experiments 2 and 3, scores to the s-else item were significantly higher in 3PP-NO than in 1PP-TO.This complementary result shows that the virtual body seen in the far extra-personal space was experienced as the body of another person rather that as one's own body, supporting the finding that no ownership is generally experienced toward the virtual body seen in the far extra-personal space. Despite this, both TT and CCT performances consistently show that actual changes in the perceived self-location took place in 3PP-NO. The distribution of $\triangle$ TubeShift in 3PP was found to be significantly larger than zero (indicating a perceived lateral shift in the perceived position of the whole body) and significantly larger than the $\Delta$ TubeShift measured in 1PP-TO (which was compatible with no lateral shift). This finding is consistent with results from previous studies of experimentally induced OBEs, which measured similar shifts of the perceived selflocation toward the virtual body using methods akin, e.g., the walking task (Lenggenhager et al., 2007) and the mental ball dropping task (Lenggenhager et al., 2009; lonta et al., 2011). Notwithstanding the evidence provided by questionnaire and TT data for an altered self-location in 3PP-NO, the CCT 
data from Experiment 2 in this condition did not provide clear evidence for integration of visual cues on the virtual body and tactile cues from the correspondent body locations on the physical body (the CCE-IE was not significantly dependent on side). This was indeed contrary to our expectation. However, it is worth noting that a strong trend $(p=0.052)$ for a significant cross-modal interaction was found in the CCE-RT data (CCE measured on response times). Additionally, only a weak trend for a significant difference across conditions was found in the CCE-IE ANOVA ( $p=0.07)$, which shows that the data do not provide clear evidence for CCT performance to be different in 3PP$\mathrm{NO}$ than in the other two conditions, where a strong VT interaction was detected. The lack of a significant CCE-IE effect in 3PP-NO may be due to fact that visual distracters were far on the left side of the visual field, with a loss of sensitivity. In this case a larger sample would be needed.Also, conflictive visuo-auditory cues in 3PP-NO (from the tube stroking) may have acted as additional confounders making the task more difficult, and/or contributing negatively to the OBE illusion itself. These speculations are supported by CCT performances in Experiment 3, in which white noise was introduced to mask visuo-auditory incongruences on a larger sample of participants. A significant difference in the CCE-IE across same and different sides was indeed found in both 1PP-TO and 3PPNO. This indicates that, during an OBE illusion, visual events experienced on the virtual body and tactile events experienced on the real body are integrated despite the dramatic spatial separation between them. It is important to notice that the feeling of another person to be present that may arise in our 3PP-NO condition (and generally in all experimental setups inducing full body illusion from a 3PP), may induce social mechanisms that affects self-processing (Serino et al., 2008), and in turns hinder the FBI. In any case, joint evidence from questionnaire (drift-left item), the CCT and the TT in the 3PP-NO condition speaks in favor of an actual shift of the perceived self-location toward the virtual body.

Summarizing, results from Experiments 2 and 3 provided the first experimental evidence that OBE and FBOI are two diverse perceptual illusions affecting different aspect of body self-consciousness. This was established by showing that both could occur independently of each other and that the two manifest themselves in different perceptual phenomena. In the general discussion we provide arguments for the different neural correlates that may be predominantly involved in each of the two illusions.

\subsubsection{FBOI DURING OBES: EVIDENCE FOR A STRONG INTERACTION OF OWNERSHIP AND SELF-LOCATION} Experiment 2 provided novel evidence on how the sense of ownership and the sense of self-location influence each other when they are concurrently manipulated. All data collected show that this was the case for the 3PP-PO condition, in which the visual perspective was dislocated from the eyes of the virtual avatar (3PP) while preserving a substantial overlap between the two bodies: participants in this condition experienced a strong FBOI over the virtual body with a concurrent significant change in the perceived self-location.

Apart from the drift-left item, questionnaire scores in this condition were statistically equal to those given in 1PP-TO. At the same time, the comparison between this condition and the 3PP-NO condition revealed the same significant differences found in the 1PP-TO vs. 3PP-NO comparison. This indicated that a strong FBOI occurred similarly in both 1PP-TO and 3PP-PO. On the other hand, TT data showed that participants experienced a strong drift in the perceived self-location. 
Furthermore, the drift was significantly stronger than that experienced in 3PP-NO, despite the fact that the actual displacement among the virtual and real bodies was much greater in the latter.

Analogously, the CCT data from the 3PP-PO condition support a significant shift in the perceived self-location. The highly significant cross-modal interaction found in 3PP-PO clearly showed that vibrotactile cues were indeed processed in an updated spatial register in which tactile events on the real body and visual events on the virtual body are associated according to a precise bodily-based topological correspondence. This result is even more remarkable when considering that, in this condition, the vibrotactile cues on the real left leg of participants were exactly collocated with the LEDs on the right leg of the virtual body, so that if no shift in the perceived self-location occurred we should have observed a strong interaction among these visuotactile cues, which was instead not observed (see inset in Figure 4B).

Summarizing, the experimental manipulation in the 3PP-PO condition induced concurrent changes in ownership and self-location. Most importantly, the latter was much stronger than the analogous change experienced in the 3PP-NO condition, indicating a strong interaction between ownership and self-location. Despite the fact that the two can be dissociated from each other, it seems that the sense of ownership experienced over a dislocated virtual body can importantly boost the experienced drift in the perceived self-location.

\section{General Discussion}

The experimental study here presented provides direct experimental evidence that the sense of ownership and the sense of self-location can be selectively manipulated in full body illusions. It was shown that it is possible to experience illusory changes in the perceived self-location during OBEs when no sense of ownership is experienced toward the virtual body. On the other during FBOI experienced toward a fully collocated virtual body, the perceived self-location was (obviously) not altered, as no conflict in the location of the two bodies was present.

The fact that OBE illusions can occur without changes in the sense of ownership and that OBE and FBOI are indeed two different perceptual illusions was previously suggested (Petkova et al., 2011b; Maselli and Slater, 2013), but to date it was not supported by experimental data.

Previous studies on OBE illusions have been primarily designed to manipulate and measure illusory changes in self-location. Despite this OBE illusions are often considered and discussed as belonging to the wider class of full body ownership illusions (e.g., Blanke, 2012; Limanowski et al., 2013). Having here shown that $\mathrm{FBOI}$ and $\mathrm{OBE}$ are different perceptual illusions is thus particularly important, because the lack of a clear distinction between the two illusions often resulted in interpreting observed changes in self-location as evidence for a sense ownership experienced toward a body seen in the far extra-personal space (Lenggenhager et al., 2007; Aspell et al., 2012).

As a second important outcome, the present study showed that when seeing a virtual body that 
overlaps only partially with the space occupied by the physical body, a strong FBOI is experience together with a significant shift in the perceived self-location toward the virtual body. This is indeed the first explicit experimental evidence for a FBOI experienced while having a third person visual perspective over a fake full body. Our data further show a positive interaction between the two components of body self-consciousness: in this "partial overlap" configuration, the experienced shift in self-location is in fact significantly larger than the one experienced in an OBE illusion. It seems then that, when both ownership and self-location are affected during a full body illusion, it is the sense of ownership that drives the sense of self-location toward the owned body.

Although our study does not provide any direct evidence for the existence of distinct neuronal correlates for $\mathrm{FBOI}$ and $\mathrm{OBEs}$, in the following we propose plausible candidates for the sets of neuronal structures that are likely involved in the two illusions.

\subsection{On the Neuronal Correlates of Experimentally Induced OBEs and Peripersonal Space}

It has been recently proposed (Blanke, 2012) that the best neural candidates for encoding the perceived shift in self-location experienced during experimentally induced OBEs are the populations of bimodal visuotactile (VT) neurons hosted in cortical and subcortical areas of the primates brain.

Bimodal visuotactile populations are characterized by tactile receptive fields that can cover small patches or large portions of the body surface, and by visual receptive fields (vRF) anchored to the body part mapped in the tactile modality (Graziano and Gross, 1993; Duhamel et al., 1998; Brozzoli et al., 2012; Huang et al., 2012). For these characteristic properties bimodal VT populations have been suggested to encode the peripersonal space (Fogassi et al., 1996): through their vRFs these populations define in fact the region of space surrounding the body in which visual stimuli may trigger somatic sensations. The main functional role of this dynamic representation of the external space in body-parts centered coordinates is to guide movements for defensive purposes and for efficient interaction with external objects (Graziano and Cooke, 2006; Sereno and Huang, 2014).

Results from single cell recording in the monkey have further shown that the vRF of bimodal visuotactile neurons is highly plastic and can expand after the active manipulation of tools (Iriki et al., 1996). This groundbreaking result provided the first robust experimental evidence that the peripersonal space is highly plastic and can be reshaped in relatively short time scales. The plasticity of bimodalVT neurons has been then advocated as the mechanism that allows healthy humans to embody external objects, like tools, in their body schema (Maravita and Iriki, 2004).

As discussed thoroughly in Section The Cross-Modal Congruency Task as a Measure of Selflocation, the cross-modal congruency task (CCT) has been shown to be a powerful psychophysical test to detect dynamic modulations of the peripersonal space during active tool use (Maravita et al., 2002), as well as during a rubber hand illusion (Pavani et al., 2000; Zopf et al., 2010). In line with these results, CCT performances in our study showed that, during experimentally induced OBEs, an actual extension (or shift) in the vRF of proximal visuotactile neurons occurs. This modulation can 
be interpreted as a modulation of the peripersonal space likely triggered by VT neural populations that maps large portions of the body, such as the legs. It is worth noting that neuronal populations with such properties have been identified in the human posterior parietal cortex (PPC) (Huang et al., 2012), and that the PPC has be shown to play a crucial role in mapping bodily signal into external space (Azañón et al., 2010).

In line with the original proposal from Blanke (2012), we argue that the dynamical changes in the VRF of visuotactile neurons with large receptive field on the body surface, is responsible for the perceived changes in self-location. We further argue that the same mechanisms may be regarded as the minimum common denominator of all OBEs. This proposal is not in conflict with the well-known implication of the TPJ in OBEs, which has been revealed in lesion analysis of patients suffering from recurrent OBEs (Blanke and Arzy, 2005), in electro-stimulation studies showing that OBEs can be systematically induced by direct stimulation of the TPJ (Blanke et al., 2002), and with fMRI performed during experimentally induced OBEs (lonta et al., 2011). The latter study showed in fact that activity in the TPJ seems to encode the perceived elevation of the whole body -rather than its position in extra-personal space- and thus it is plausible that the TPJ implication in OBEs occurs at higher level in the hierarchical processing of sensory signals, in which a further integration is operated to combine visuotactile cues with vestibular and auditory information (Lopez et al., 2008).

\subsection{Different Representations for the Sense of OWnership and Self- LOCATION}

Our study has shown that OBEs can occur with or without an associated ownership illusion. This implies that the dynamic changes in the peripersonal space associated with OBEs may be coupled or not with the activity of a larger and more complex network of neuronal populations that give rise to the sense of ownership during FBOI. Imaging studies have shown that the illusion of ownership for both body parts (RHI) and full bodies involves a complex network of brain areas that is still not well established and controversial. Depending on the experimental design and the imaging technique adopted, the ownership illusion has been found to correlate with activity in several brain areas: ventral premotor cortex (vPMc) and posterior parietal cortex (PPc) (Ehrsson et al., 2004; Petkova et al., 2011a), primary somatosensory cortex (Limanowski et al., 2013; Shokur et al., 2013), the extrastriate body area (EBA) (Limanowski et al., 2013); and, at subcortical level, the insula (Tsakiris et al., 2007; Limanowski et al., 2013). Recent theoretical accounts for body ownership have stressed the role of multimodal hierarchical processing of sensory and motor signals from the body (e.g., Hohwy, 2007; Ehrsson, 2011; Moseley et al., 2012; Limanowski and Blankenburg, 2013). Although a review of these works is beyond the scope of the present work, we want to stress here the potential contribution that our results may bring about.

The present study has highlighted, in particular, the fundamental role of visuo-proprioceptive correlations in driving FBOI. In a previous study we showed how congruent visuo-proprioceptive correlations from a (realistic) virtual body and the physical body could be sufficient to elicit a FBOI with no need for additional multimodal correlations (Maselli and Slater, 2013). The current 
study provides more stringent evidence for the effective coupling of visual signals from the virtual body and proprioceptive signals from the real one, that is established during FBOI. It was in fact shown that proprioceptive information about the body posture can be significantly modulated by seeing a change in the posture of the virtual body, even when the physical body does not move. Furthermore, we found that if visual and proprioceptive cues about the body are not in severe conflict (i.e., when a partial spatial overlap of the virtual and real bodies is preserved) a strong FBOI can be experienced. We additionally found that in this case the FBOI has the effect of strengthening the recalibration of tactile sensations toward the virtual body, which is characteristic of OBEs. These results strongly support the earlier proposals (Moseley et al., 2012; Maselli and Slater, 2013) suggesting that a fundamental node of the complex network of brain areas involved in ownership illusions is the neural population homologous to the one hosted in area 5 of the monkey parietal cortex, that integrates visual and proprioceptive signals to encode limb position (Graziano et al., 2000).

\section{SUMMARY}

We have presented an experimental study conducted with the support of virtual reality, with the aim of exploring the relation between two of the important components of body self-consciousness: ownership and self-location. Our results provide direct experimental evidence that the sense of ownership and the sense of self-location can be selectively manipulated in experimentally induced full body illusions. It was shown that FBOls and OBEs could occur independently of each other, affecting ownership and self-location respectively. It was also shown that the two illusions could co-occur affecting concurrently ownership and self-location; our results suggest that in these cases illusory changes in ownership and self-location have a synergic interaction.

We discussed how our results support the recent proposal suggesting that self-location is strictly related with a reshaping of the visual receptive field of proximal visuotactile neurons mapping large portions of the body's surface. On the other hand, we discussed how our results support the need for a driving activation of visuo-proprioceptive neuronal populations in full body ownership illusions.

Finally, our results moderate previous conclusions about the role of the visual perspective in ownership illusions. It was in fact shown that a FBOI could be experienced from a 3PP when the virtual body preserved a partial spatial overlap with the physical body. 


\section{REFERENCES}

Aspell, J. E., Lenggenhager, B., and Blanke, O. (2009). Keeping Duhamel, J., Colby, C. L., and Goldberg, M. E. (1998). Ventral in touch with one's self: multisensory mechanisms of self- intraparietal area of the macaque: congruent visual and somatic consciousness. PLoS ONE 4:e6488 response properties J. Neurophysiol. 79, 126-136

Aspell, J. E., Lenggenhager, B., and Blank, O. (2012). Ehrsson, H. H. (2007). The experimental induction of out-of"Multisensory perception and bodily self-consciousness from body experiences. Science 317, 1048 out-of-body to inside-body experience," in The Neural Bases of Multisensory Processes, eds M. M. Murray and M.T.Wallace Ehrsson, H. H. (2011). "The concept of body ownership and its (Boca Raton, FL: CRC Press), 467-481.

Azañón, E., Longo, M. R., Soto-Faraco, S., and Haggard, P. Press), 775-792. (2010). The posterior parietal cortex remaps touch into external space. Curr. Biol. 20, 1304-1309

Ehrsson, H. H., Spence, C., and Passingham, R. E. (2004). That's my hand! activity in premotor cortex reflects feeling of Blanke, O. (2012). Multisensory brain mechanisms of bodily self- ownership of a limb. Science 305, 875-877 consciousness. Nat. Rev. Neurosci. 13, 556-571

Fogassi, L., Gallese,V., Fadiga, L., Luppino, G., Matelli, M., and Blanke, O., and Arzy, S. (2005). The out-of-body experience: Rizzolatti, G. (1996). Coding of peripersonal space in inferior disturbed self-processing at the temporo-parietal junction. premotor cortex (area F4). J. Neurophysiol. 76, 141-157 Neuroscientist 11,16-24

Graziano, M. S. A., and Cooke, D. F. (2006). ParietoBlanke, O., Landis, T., Spinelli, L., and Seeck, M. (2004). Out- frontal interactions, personal space, and defensive behavior. of-body experience and autoscopy of neurological origin. Brain Neuropsychologia 44, 845-859 $127,243-258$

Graziano, M. S.A., Cooke, D. F., and Taylor, C. S. (2000). Coding Blanke, O., and Metzinger, T. (2009). Full-body illusions and the location of the arm by sight. Science 290,1782-1786 minimal phenomenal selfhood. Trends Cogn. Sci. 13, 7-13

Graziano, M. S.A., and Gross, C. G. (1993). A bimodal map of Blanke, O., and Mohr, C. (2005). Out-of-body experience, space: somatosensory receptive fields in the macaque putamen heautoscopy, and autoscopic hallucination of neurological with corresponding visual receptive fields. Exp. Brain Res. 97, origin implications for neurocognitive mechanisms of corporeal 96-109 awareness and self-consciousness. Brain Res. Brain Res. Rev. 50,184-199

Grissom, R. J., and Kim, J. J. (2012). Effect Sizes for Research: Univariate and Multivariate Applications, 2nd Edn, Vol. 44. New Blanke, O., Ortigue, S., Landis, T., and Seeck, M. (2002). York, NY:Taylor and Francis Group, LLC.

Stimulating illusory own-body perceptions. Nature 419, 269270

Guterstam, A., and Ehrsson, H. H. (2012). Disowning one's seen real body during an out-of-body illusion. Conscious. Cogn. Botvinick, M., and Cohen, J. (1998). Rubber hands "feel" touch 21, 1037-1042 that eyes see. Nature 391, 756

Hohwy, J. (2007). The Sense of self in the phenomenology of Brozzoli, C., Gentile, G., and Ehrsson, H. H. (2012). That's agency and perception. Psyche (Stuttg) 13, 1-20. near my hand! parietal and premotor coding of hand-centered space contributes to localization and self-attribution of the hand. J. Neurosci. 32, 14573-14582

Huang, R.-S., Chen, C., Tran, A.T., Holstein, K. L., and Sereno, M. I. (2012). Mapping multisensory parietal face and body areas in humans. Proc. Nat. Acad. Sci. U.S.A. 109, 18114-18119

Bruyer, R., and Brysbaert, M. (2011). Combining speed and accuracy in cognitive. Psychol. Belgica 51, 5-13

Ionta, S., Heydrich, L., Lenggenhager, B., Mouthon, M., Fornari, E., Chapuis, D., et al. (2011). Multisensory mechanisms Driver, J., and Spence, C. (1998a). Attention and the crossmodal in temporo-parietal cortex support self-location and first-person construction of space. Trends Cogn. Sci. 2, 254-262 perspective. Neuron $70,363-374$

Driver, J., and Spence, C. (1998b). Cross-modal links in spatial Iriki,A.,Tanaka, M., and Iwamura,Y. (1996). Coding of modified attention. Philos. Trans. R. Soc. Lond. B. Biol. Sci. 353, 1319- body schema during tool use by macaque postcentralneurones. 1331 Neuroreport 7, 2325-2330 
Neurosci. Biobehav. Rev. 36, 34-46

Kennett, S., Eimer, M., Spence, C., and Driver, J. (2001).

Tactile-visual links in exogenous spatial attention under different Moseley, G. L., Olthof, N., Venema, A., Don, S., Wijers, M., postures: convergent evidence from psychophysics and ERPs. J. Gallace, A., et al. (2008). Psychologically induced cooling of Cogn. Neurosci. 13, 462-478

a specific body part caused by the illusory ownership of an artificial counterpart. Proc. Nat.Acad. Sci. U.S.A. 105, 13169-

Kokkinara, E., and Slater, M. (2014). Measuring the effects 13173

through time of the influence of visuomotor and visuotactile synchronous stimulation on a virtual body ownership illusion. Perception 43, 43-58

Pavani, F., Spence, C., and Driver, J. (2000). Visual capture of touch: out-of-the-body experiences with rubber gloves. Psychol. Sci. 11, 353-359

Lenggenhager, B., Mouthon, M., and Blanke, O. (2009). Spatial aspects of bodily self-consciousness. Conscious. Cogn. Peck, T. C., Seinfeld, S., Aglioti, S. M., and Slater, M. (2013). $18,110-117$ Putting yourself in the skin of a black avatar reduces implicit racial bias. Conscious. Cogn. 22, 779-787

Lenggenhager, B., Tadi, T., Metzinger, T., and Blanke, O. (2007). Video ergo sum: manipulating bodily self-consciousness. Perneger,T.V.(1998). What's wrong with Bonferroni adjustments. Science 317, 1096-109 BMJ 316, 1236-1238.

Limanowski, J., and Blankenburg, F. (2013). Minimal self- Petkova, V. I., Björnsdotter, M., Gentile, G., Jonsson, T., Li, models and the free energy principle. Front. Hum. Neurosci. T.-Q., and Ehrsson, H. H. (2011a). From part- to whole-body 7:547 ownership in the multisensory brain. Curr. Biol. 21,1118-1122

Limanowski, J., Lutti, A., and Blankenburg, F. (2013). The Petkova, V. I., and Ehrsson, H. H. (2008). If I were you: extrastriate body area is involved in illusory limb ownership. perceptual illusion of body swapping. PLoS ONE 3:e3832. doi: Neuroimage 86, 514-524 10.1371/journal.pone.0003832

Lloyd, D. M., Shore, D. I., Spence, C., and Calvert, G. A. Petkova, V. I., Khoshnevis, M., and Ehrsson, H. H. (2011b). (2003). Multisensory representation of limb position in human The perspective matters! Multisensory integration in egopremotor cortex. Nat. Neurosci. 6,17-18

Longo, M. R., Schüür, F., Kammers, M. P. M., Tsakiris, M., and Haggard, P. (2008). What is embodiment? A psychometric Pomés,A., and Slater, M. (2013). Drift and ownership toward a approach. Cognition 107, 978-998 distant virtual body. Front. Hum. Neurosci. 7:908

Lopez, C., Halje, P., and Blanke, O. (2008). Body ownership Salomon, R., Lim, M., Pfeiffer, C., Gassert, R., and Blanke, and embodiment: vestibular and multisensory mechanisms. Clin. O. (2013). Full body illusion is associated with widespread skin Neurophysiol. 38, 149-161 temperature reduction. Front. Behav. Neurosci. 7:65

Makin,T. R., Holmes, N. P., and Ehrsson, H. H. (2008). On the Salomon, R., van Elk, M.,Aspell, J. E., and Blanke, O. (2012). I other hand: dummy hands and peripersonal space. Behav. feel who I see:visual body identity affects visual-tactile integration Brain Res. 191,1-10 in peripersonal space. Conscious. Cogn. 21, 1355-1364

Maravita,A., and Iriki,A. (2004).Tools for the body (schema). Sereno, M. I., and Huang, R.-S. (2014). Multisensory maps in Trends Cogn. Sci. 8, 79-86 parietal cortex. Curr. Opin. Neurobiol. 24, 39-46

Maravita, A., Spence, C., and Driver, J. (2003). Multisensory Serino, A., Alsmith, A., Costantini, M., Mandrigin, A., integration and the body schema: close to hand and within Tajadura-jimenez, A., and Lopez, C. (2013). Bodily ownership reach. Curr. Biol. 13, R531-R539 and self-location: components of bodily. Conscious. Cogn. 22, $1239-1252$

Maravita, A., Spence, C., Kennett, S., and Driver, J. (2002).

Tool-use changes multimodal spatial interactions between vision Serino, A., Pizzoferrato, F., and Làdavas, E. (2008). Viewing a and touch in normal humans. Cognition 83, B25-B34

Maselli,A., and Slater, M. (2013). The building blocks of the full body ownership illusion. Front. Hum. Neurosci 7:83 face (especially one's own face) being touched enhances tactile perception on the face. Psychol. Sci. 19, 434-438

Shapiro, S. S., and Wilk, M. B. (1965). An analysis of variance test for normality (complete samples). Biometrica 52, 591-611

Moseley, G. L., Gallace, A., and Spence, C. (2012). Bodily

illusions in health and disease: physiological and clinical Shokur, S., O'Doherty, J. E.,Winans, J.A., Bleuler, H., Lebedev, perspectives and the concept of a cortical "body matrix." M.A., and Nicolelis, M. A. L. (2013). Expanding the primate 
body schema in sensorimotor cortex by virtual touches of an avatar. Proc. Nat. Acad. Sci. U.S.A. 110, 15121-15126

Shore, D. I., Barnes, M. E., and Spence, C. (2006). Temporal aspects of the visuotactile congruency effect. Neurosci. Lett. 392, 96-100

Slater, M., Spanlang, B., Sanchez-Vives, M. V., and Blanke, O. (2010). First person experience of body transfer in virtual reality. PLoS ONE 5:e10564

Spence, C., Kingstone, A., Shore, D. I., and Gazzaniga, M. S. (2001). Representation of Visuotactile space in the split brain. Psychol. Sci. 12, 90-93

Spence, C., Pavani, F., and Driver, J. (2004). Spatial constraints on visual-tactile cross-modal distractor congruency effects. Cogn. Affect. Behav. Neurosci 4, 148-169

Spence, C., Pavani, F., Maravita, A., and Holmes, N. P. (2008). "Multi-sensory interactions," in Haptic Rendering: Foundations, Algorithms, and Applications, eds M. C. Lin and M.A. Otaduy (Wellesley, MA:AK Peters), 21-52.

Townsend, J.T., and Ashby, F.G.(1983). The stochastic Modeling of Elementary Psychological Processes. The American Journal of Psychology, Vol. 98. Cambridge: Cambridge University Press.

Tsakiris, M., Hesse, M. D., Boy, C., Haggard, P., and Fink, G. R. (2007). Neural signatures of body ownership: a sensory network for bodily self-consciousness. Cereb. Cortex 17, 2235-2244

Tsakiris, M., Longo, M. R., and Haggard, P. (2010). Having a body versus moving your body: neural signatures of agency and body-ownership. Neuropsychologia 48, 2740-2749

Tukey, J.Ws. (1977). Exploratory Data Analysis. Reading, MA: Addison-Wesley.

Zopf, R., Savage, G., and Williams, M. A. (2010). Crossmodal congruency measures of lateral distance effects on the rubber hand illusion. Neuropsychologia 48, 713-725

Zopf, R.,Truong, S., Finkbeiner, M., Friedman, J., and Williams, M.A. (2011). Viewing and feeling touch modulates hand position for reaching. Neuropsychologia 49, 1287-1293 



\section{CHAPTER 7}

\section{Allocentric and egocentric}

\section{MANIPULATIONS OF THE SENSE OF SELF-LOCATION IN}

\section{FULL-BODY ILLUSIONS AND THEIR RELATION WITH THE}

\section{SENSE OF BODY OWNERSHIP}

Related publication:

Allocentric and egocentric manipulations of the sense of self-location in full-body illusions and their relation with the sense of body ownership.

Maselli,A.

Cognitive Processes. 16, 2, 2015 


\section{Abstract}

Self-location refers to the experience of occu- pying a given position in the environment. Recent research has addressed the sense of self-location as one of the key components of self-consciousness, together with the experience of owning the physical body (ownership). Experimentally controlled full-body illusions proved to be valuable research tools to study these components and their interaction, and to explore their underlying neural underpinning. The focus of this manuscript is to provide a close look into the nuances of different illusory experiences affecting the sense of selflocation and to examine their relation to the concurrent experienced sense of body ownership. On the basis of previous reviewed studies, it is proposed that the sense of self-location may be regarded as the blending of two parallel representations: the abstract allocentric coding of the position occupied in the environment, mainly associated with visual-perspective, and the egocentric mapping of somatosensory sensations into the external space, mainly associated with peripersonal space. Open questions to be addressed by future research are further addressed. 


\section{INTRODUCTION}

\section{Spatial AND bodily ASPECTS Of SELF-LOCATION}

Two main spatial components are contemplated in recent accounts of self-consciousness: self-location, intended as the perceived position of the body in space, and the visual-perspective, defined as the point from which visual information from the environment is gathered (Blanke and Metzinger 2009). In normal conditions, both self-location and visual-perspective coincide with the position of the physical body. This tight coupling can be temporally disrupted during neurological or experimentally induced out-of-body experiences, in which subjects report the sensation to be located outside their body and to perceive the world from a visual-perspective incongruent with the position of the physical body (Blanke 2012).

In out-of-body illusion experiments, the visual-perspective is decoupled from the position of the seen body by using stereo goggles that project an immersive view of the environment from an arbitrary location. In the presence of environmental landmarks, such as walls or furniture, the mere manipulation of visual-perspective could engender an abstract allocentric representation of self-location within the seen room (Barry and Burgess 2014), as demonstrated during navigation in virtual environments (Hassabis et al. 2009).

Manipulations of the visual-perspective in out-of-body illusion experiments entail a third-person view of the real body within the experimental room, or of a fake body in a virtual environment. Furthermore, visuotactile stimulation is delivered through a moving rod. The processing of similar semantic and multisensory information can trigger modulations in the activity of bimodal visuotactile populations and other visuotactile subpopulations responding also to proprioceptive, semantic (Graziano et al. 2000) or vestibular (Bremmer et al. 2002) stimuli. These multisensory populations establish a somatosensory connection to visual stimuli in the environment and are regarded as the main encoders of peripersonal space (Graziano and Gross 1993; Fogassi et al. 1996).

Within the context of full-body illusions, it shall thus be advantageous to consider the sense of self-location as the blending of two parallel representations: the abstract allocentric coding of the position occupied in the environment, mainly associated with the visual-perspective, and the egocentric mapping of somatosensory sensations into the external space, mainly associated with peripersonal space.

\section{MANIPULATIONS OF SELF-LOCATION IN OUT-OF-BODY ILLUSIONS}

Experimental out-of-body illusions allow investigating the multisensory and neurophysiological basis of self-location. Two main paradigms have been exploited hitherto. Here I review the most relevant studies rationalizing them on the basis of the multisensory and neurophysiological processes involved. Common to the two paradigms is the use of immersive goggles to prevent direct vision of the real body and provide participants with a third-person visual-perspective over their own body (filmed from a distance; e.g. Ehrsson 2007) or over a virtual body (lonta et al. 2011). This visual-perspective's 
manipulations can be modulated by providing or not environmental landmarks: when participants see their body as filmed from a different position within the experimental room, they have a new egocentric visual-perspective over the known environment that automatically triggers mechanisms of allocentric remapping of self-location (Barry and Burgess 2014). In the case in which no landmark is present, like the case in which the avatar is the only visible element (lonta et al. 2011), there are no spatial cues for activating allocentric remapping of self-location.

Another common ingredient is the delivery of visuo-tactile stimulation through a moving object. The difference between experimental paradigms concerns the spatial congruence of such stimulation. In one case, a rod is used to stroke the real hidden body on the front, while a similar rod is seen moving in temporal synchrony and with spatial patterns that match the felt touch in the egocentric visual-perspective (Ehrsson 2007). Alternatively, the touch is delivered on the back of the participant that sees the stick moving synchronously on the back of the body in front, thus in spatial incongruence with the felt touch (Lenggenhager et al. 2007).Accordingly, in the front-stroking there is no visuotactile conflict: the touch is seen where it is felt, even if empty space is seen instead of the real body (Ehrsson 2007), or the latter is replaced by a fake one (Guterstam et al. 2015). The backstroking involves instead a spatial conflict: no visual counterpart of the felt touch is seen where expected; nevertheless, the precise temporal synchronicity and the corresponding spatial patters in body-centred coordinates may trigger an aberrant coupling among somatosensory sensations from the real body and visual cues on the seen body, similarly to the case of tool incorporation (Maravita and Iriki 2004).

\section{NeURAL CORRELATES OF SELF-LOCATION IN OUT-OF-BODY ILLUSIONS}

Which are the neurophysiological and perceptual correlates of front-stroking and back-stroking paradigms?

A recent imaging study adopting the front-stroking paradigm revealed the implication of a set of brain regions including hippocampus, posterior cingulate cortex (PCC), retrosplenial cortex (RSC) and intraparietal sulcus (IPS; Guterstam et al. 2015). The induced changes in self-location seem thus to be mostly related to the recalibration of the allocentric representation of self-location in the environment, triggered as the artificial visual-perspective is nourished with congruent visuotactile signals. The hippocampus hosts in fact neural populations that encode mnemonic allocentric representations (Hassabis et al. 2009), while the PCC and RSC are involved in the translation of egocentric-to-allocentric spatial representations (Vann et al. 2009). In the altered self-location experience, the role of PCC and RSC would be to translate body-centred, head-centred and retinotopic spatial representations encoded in the IPS, into allocentric spatial representations stored in the hippocampus (Guterstam et al. 2015 and references therein). Subjective scores to selflocation assessments support this view, revealing that the illusory self-location corresponds to the position of the visual-perspective (Guterstam and Ehrsson 2012; Guterstam et al. 2015).

In back-stroking out-of-body experiments, self-location is altered in a more subtle fashion. The temporally synchronous but spatially incongruent visuotactile stimulation can in fact induce a spatial dissociation between visual-perspective and self-location, with the latter shifted towards the seen body. This was found in both allocentric (Lenggenhager et al. 2007) and egocentric 
(Lenggenhager et al. 2009) representations and is associated with strengthened interactions of visual cues on the fake body with tactile cues on the real one (Aspell et al. 2009; Maselli and Slater 2014). The remapping of the visual receptive field of visuotactile populations, which would extend or shift to encompass the seen body, has been proposed as the origin of the detachment of selflocation from visual-per-spective (Blanke 2012). However, although this proposal is grounded in several behavioural and neurophysiological data (Maravita and Iriki 2004), there is no direct evidence in its support.

Results from brain-recording studies are still fragmented. When applying the back-stroking paradigm to supine participants, TPJ activation patterns suggest the encoding of the perceived elevation (lonta et al. 2011), while TPJ's connectivity patterns reveal the dominance of right-hemispheric networks in modulating self-location and visual-perspective's direction (lonta et al.2014). These results are consistent with the multisensory nature of TPJ populations (integrating vestibular, visual, tactile and auditory signals) and the well-known implication of this region in neurological out-of-body experiences (Blanke and Mohr 2005). No major involvement of the TPJ was instead found when inducing the illusion in standing participants, as no elevation sensations were triggered. In this configuration, changes in self-location were correlated with decreased activity in the medial-prefrontal cortex (Lenggenhager et al. 2011), suggesting that the processing of visuotactile conflicts may be sufficient for detaching self-location from visual-perspective, independently of anomalous vestibular activity.

\section{THE SENSE OF BODY OWNERSHIP DURING OUT-OF-BODY ILLUSIONS}

Body ownership refers to the experience that the physical body belongs to the self. Experimental manipulations similar to those adopted for out-of-body illusions can project the sense of ownership to fake bodies (for a review see Kilteni et al. 2015). While ownership illusions are easily experienced when seeing the fake body in spatial overlap with the real body (Maselli and Slater 2013; Petkova and Ehrsson 2008), the extent to which ownership can be experienced over a body seen from a detached visual-perspective is under debate (Serino et al. 2013; Maselli and Slater 2014).

As for self-location, different out-of-body paradigms have different impacts on the sense of ownership. In front- stroking setups, subjects experience disownership of their body seen from a distance (Guterstam and Ehrsson 2012): it is as if the processing of visuotactile cues congruent with the novel visual-perspective, locks self-location to visual-perspective and deprives the seen body of accessible somatosensory experiences. Complementing the altered visual-perspective with the view of a spatially congruent fake body may further bind self-location to visual-perspective and enhance disownership of the self-body seen from outside (Guterstam et al. 2015).

In the back-stroking case, the multisensory coupling between the real and the seen body can elicit some degree of ownership over the latter, even if not systematically (Serino et al. 2013). In fact, changes in self-location are not sufficient to trigger ownership over the fake body, even when involving a remapping of tactile cues towards distant external space, i.e. on the seen fake body (Maselli and Slater 2014). Interestingly, when the fake body is displaced from the real one while keeping partial spatial overlap with it, synchronous visuotactile stimulation triggers changes in both self-location and body ownership, with reciprocal synergic interactions among the two (Maselli and Slater 2014). This result speaks in favour of the major role of visuoproprioceptive populations in driving the sense of 
body ownership (Maselli and Slater 2013).

\section{CONCLUSIONS AND FUTURE DIRECTIONS}

In this manuscript I have argued that, in the context of full-body illusions, self-location can be advantageously regarded as the blending of two parallel spatial representations: an abstract allocentric representation, mainly associated with visual-perspective, and the egocentric mapping of somatosensory sensations into the external space, mainly associated with peripersonal space. According to the specific experimental paradigm adopted to induce out-of-body illusions, these representations can be selectively or simultaneously altered, with different implications for the sense of ownership.

Future studies are needed to establish how the two representations are integrated to results in the subjective experience of being located in a given position in space. In fact, the two resonance imaging studies conducted to date have explicitly focused on either one or the other aspect. In Guterstam et al. (2015) self-location was locked to the visual-perspective, and the latter was manipulated so to provide anomalous visual cues on environmental landmarks and thus to trigger an allocentric remapping of self-location. In lonta et al. (2011) no landmarks were available and changes in selflocation mainly relied on the presentation of conflicting multisensory bodily cues, detaching selflocation from visual-perspective through anomalous visuotactile correlations.

Another important missing piece of the puzzle is a direct evidence in support of the proposal that the reshaping of visual receptive field of visuotactile populations may be responsible for decoupling self-location from visual-perspective. It would be further interesting to assess whether the suggested involvement of visuotactile subpopulations responding also to vestibular signal is necessary (Blanke 2012) or optional (Maselli and Slater 2014) for eliciting illusory changes in self-location.

Finally, experimental paradigms for out-of-body illusions always included visuotactile stimulation. Nevertheless, the novel design proposed in Guterstam et al. (2015) suggests the possibility to investigate illusory changes in self-location while eliminating confounds associated with visuotactile processing. In their design, the altered visual-perspective over the scanning room was complemented with the view of a spatially congruent fake body. This configuration was shown to be sufficient for eliciting full-body ownership illusions (Maselli and Slater 2013), which in turn could drive self-location to the position of the visual-perspective with no need for visuotactile triggers.

Addressing these among other questions in future studies will provide important advances in the fields of self-consciousness and spatial cognition. 


\section{REFERENCES}

Aspell JE, Lenggenhager B, Blanke O (2009) Keeping in touch with one's self: multisensory mechanisms of self-consciousness. PLoS ONE 4:e6488.

Barry C, Burgess N (2014) Neural mechanisms of self-location. Curr Biol 24:R330-R339.

Blanke $O$ (2012) Multisensory brain mechanisms of bodily selfconsciousness. Nat Rev Neurosci 13:556-571.

Blanke O, Metzinger T (2009) Full-body illusions and minimal phenomenal selfhood. Trends Cogn Sci 13:7-13.

Blanke O, Mohr C (2005) Out-of-body experience, heautoscopy, and autoscopic hallucination of neurological origin Implications for neurocognitive mechanisms of corporeal awareness and self-consciousness. Brain Res Brain Res Rev 50:184-199.

Bremmer F, Duhamel JR, Ben Hamed S, GrafW (2002) Visual- vestibular interactive responses in the macaque ventral intraparietal area (VIP). Eur J Neurosci 16:1569-1586.

Ehrsson HH (2007) The experimental induction of out-of-body experiences. Science 317:1048.

Fogassi L, Gallese V, Fadiga L et al (1996) Coding of peripersonal space in inferior premotor cortex (area F4). J Neurophysiol 76:141-157

Graziano MSA, Gross CG (1993) A bimodal map of space: somatosensory receptive fields in the macaque putamen with corresponding visual receptive fields. Exp Brain Res 97:96-109

Graziano MSA, Cooke DF, Taylor CS (2000) Coding the location of the arm by sight. Science 290:1782-1786

Guterstam A, Ehrsson HH (2012) Disowning one's seen real body during an out-of-body illusion. Conscious Cogn 21:1037-1042.

Guterstam A, Bjo "rnsdotter M, Gentile G, Ehrsson HH (2015). Posterior cingulate cortex integrates the senses of self-location and body ownership. Curr Biol 25:1-10.

Hassabis D, Chu C, Rees G et al (2009) Decoding neuronal ensembles in the human hippocampus. Curr Biol 19:546-554.

lonta S, Heydrich L, Lenggenhager B et al (2011) Multisensory mechanisms in temporo-parietal cortex support self-location and first-person perspective. Neuron 70:363-374.

lonta S, Martuzzi R, Salomon R, Blanke O (2014) The brain network reflecting bodily self-consciousness: a functional connectivity study. Soc Cogn Affect Neurosci.

Kilteni K, Maselli A, Koerding KP and SlaterM(2015) Over my fake body: body ownership illusions for studying the multisensory basis of own-body perception. Front Hum Neurosci
$9: 141$

Lenggenhager B, Tadi T, Metzinger T, Blanke $O$ (2007) Video ergo sum: manipulating bodily self-consciousness. Science 317:1096-1099.

Lenggenhager B, Mouthon M, Blanke O (2009) Spatial aspects of bodily self-consciousness. Conscious Cogn 18:110 117.

Lenggenhager B, Halje P, Blanke O (2011) Alpha band oscillations correlate with illusory self-location induced by virtual reality. Eur J Neurosci 33:1935-1943.

Maravita A, Iriki A (2004) Tools for the body (schema). Trends Cogn Sci 8:79-86.

Maselli A, Slater M (2013) The building blocks of the full body ownership illusion. Front Hum Neurosci 7:83.

Maselli A, Slater M (2014) Sliding perspectives: dissociating ownership from self-location during full body illusions in virtual reality. Front Hum Neurosci 8:1-19.

Petkova VI, Ehrsson HH (2008) If I were you: perceptual illusion of body swapping. PLoS ONE 3:e3832.

Serino A, Alsmith A, CostantiniMet al (2013) Bodily ownership and self-location: components of bodily self-consciousness. Conscious Cogn 22:1239-1252.

Vann SD, Aggleton JP, Maguire EA (2009) What does the retrosplenial cortex do? Nat Rev Neurosci 10:792-802. 

CHAPTER 8

\section{General Discussion}





\section{GeneralDiscussion}

The research presented in this thesis aimed at providing new insights and a comprehensive view of the multisensory underpinning of full-body illusions. Multisensory integration has been addressed as the causal root of bodily illusions since the first report of the rubber hand illusion (Botvinick and Cohen, 1998). Since then, experimentally induced bodily illusions have been endowed with the status of unique tools for studying the otherwise elusive neural and multisensory basis of selfbody perception (Botvinick, 2004; Graziano and Botvinick, 2002). Indeed, a large number of studies provided important insights into the relation between the processing of body-related sensory and semantic stimuli on one hand, and self-perception with its different facets and corresponding neural underpinnings on the other. Despite this collective effort, results from independent studies were not always consistent with each other, and a common framework able to encompass the outcome of the numerous and diverse studies carried out during more than ten years of research was still missing.

The work presented in this thesis tackled some of the open-issues emerging from controversial results in the literature. We directly faced questions like:Which is the role of visuotactile stimulation in ownership illusion? Are congruent visuotactile information a necessary ingredient to experience ownership over a fake body? If not, which are the alternative mechanisms that can trigger such illusions? On a different side: Which is the role of visual perspective? Is it possible to experience ownership over a fake body or body-part seen from a 3PP? If this is not the case, why? Which are the different multisensory mechanisms that set apart full body ownership illusions from illusory out-of-body experiences? Although some of these questions may seem naïve, an astonishing lack of consensus was there. We have addressed these and other related questions for the specific case of fullbody ownership illusions (FBOIs), with particular focus on how different experimental manipulations used to elicit full-body illusions have an impact on the experienced sense of body ownership and self-location. Our approach was mainly based on experimental setups integrating Immersive Virtual Reality, motion capture systems, physiological measures and psychophysical assessments. This experimental work, together with a thoughtful effort for modelling and encompassing results from the relevant literature in a coherent overall framework, provided several new insights in the field. The present chapter reviews and discusses the most relevant findings.

\section{THE KEY ROLE OF VISUOPROPTIOCEPTIVE BINDING IN OWNERSHIP ILLUSIONS}

In the attempt to pinpoint the minimal combinations of multisensory components contributing to the sense of body ownership and analyze their mutual interaction, the work presented Chapter 2 brought about several noticeable results. First, we showed for the first time that the mere exposure to a static view on a realistic collocated virtual body could elicit FBOls. This confirmed that, differently from what previously stated (Petkova and Ehrsson, 2008), synchronous visuotactile stimuli are not necessary for eliciting ownership over a fake body, but could be replaced by other forms of dynamic multisensory stimuli, like spatiotemporally congruent visuovestibular or visuomotor stimuli (González-Franco et al., 2010; Sanchez-Vives et al., 2010; Slater et al., 2010). As a novelty, it implied that a much simpler form of multisensory manipulation, static congruent visuoproprioceptive stimuli, could be an effective trigger by itself. This provided a novel evidence for the crucial role of visuoprioceptive integration in building up the sense of body ownership. 
The role of visuoproprioception binding in establishing the illusion was largely overlooked in previous studies, probably due to the intrinsic limitation of experimental setups alike to the one used in classic rubber hand illusion (RHI), in which the physical object used as surrogate body part had to be necessarily placed aside the corresponding body part. Under similar configurations visual and proprioceptive information about the body are incongruent, so that visuotactile (and/ or visuomotor) congruent stimulations were actually necessary triggers for the illusion. A general view got therefore established that considered the multisensory processing of trains of multimodal stimuli as the key mechanisms for triggering changes in the sense of ownership. In this view, once triggered, the sense of ownership over the fake body would have an impact on proprioception, driving the spatial coding of the self-body towards its seen surrogate (e.g. Makin et al., 2008), an effect robustly assessed via the proprioceptive drift measure. In this respect, our result brought a discontinuity in the narrative, shedding light on the primary role of visuoproprioceptive binding in shaping the sense of body ownership. Not only we showed that seeing a fake body exactly where the real hidden body is can by itself elicit ownership illusions, but we point at visuoproprioceptive binding as the key minimal component underlying the illusory experience of ownership towards external objects.

This finding has been replicated in following independent studies, and/or taken as the basis for novel experimental setups exploiting the possibility to induce ownership illusions while avoiding the possible interference of dynamic stimulation like visuotactile triggers (Martini et al., 2015; Nierula et al., 2017; Tieri et al., 2015). Building on this result, in a later study (presented in Chapter 6), we introduced a novel behavioral task to test illusory ownership over a fake body. The task consisted in a proprioceptive assessment about the self-body posture (in our case in pointing to the position of the ankles) in absence of vision, to be done before and after the experimental manipulation. Right before the second proprioceptive assessment and after the experimental manipulation, participants viewed the virtual body slightly moving into a new posture while instructed to not move. The rationale was that, for those participants who experienced ownership over the virtual body, the induced visuoproprioceptive binding between the real and the virtual body would induce a recalibration of the felt position when seeing the virtual body moving, even in absence of actual movement (Maselli and Slater, 2014). Results supported this prediction, on the one hand confirming that a tight visuoproprioceptive binding is established during body ownership illusions, and on the other proving the proposed test to be a reliable behavioral assessment for illusory ownership.

The study presented in Chapter 2 provided further relevant contributions. As from our main aim to pinpoint the "building blocks" of the ownership illusion, it provided a novel view on how the different multisensory components contributing to the illusion interact reciprocally. The most relevant findings were the following: 1) when a $\mathrm{FBOI}$ is established via visuoproprioceptice binding, further exposure to multisensory stimulations modulates the strength of the illusion, with a reinforcing or damping effect depending on their congruency; 2) the level of realism of the virtual body modulates the strength of the illusion; 3) having a 1PP over the fake body is a necessary condition for ownership illusions to occur. In discussing these results, we proposed a "basic model" for ownership illusions, in which we pinpointed the basic constraints that should be satisfied when combining different multisensory and semantic information about the body, from the real body and its fake counterpart, in order for illusory ownership to emerge. In a later study, presented in 
Chapter 5, these ideas were further elaborated, presented more systematically in the context of the relevant literature, and further formulized within a proposal of a causal inference computational model.

\section{A CAUSAL INFERENCE MODEL FOR OWNERSHIP ILLUSIONS}

The model presented in Chapter 5 has been worked out in the Bayesian inference framework and for the representative case of the classic RHI. Our approach was to look for a conceptual framework for modelling how our nervous system differentiate between external objects, including others bodies, and the self-body with its body parts. According to the model, the illusion would take place depending on the probability that all the sensory information available (visual from the rubber hand and somatosensory from the real hand) is assumed to have the same one origin, the own hand, in contrast to the case of having two independent origins, the real hand and the rubber hand. This probability could be estimated in terms of the likelihood of the available sensory information under the assumption of a common origin, and the prior belief about the existence of more than one hand. Beside the details of the formulation, the model allows to account for the effect of different types of multisensory and semantic components potentially contributing to the illusion, also accounting for their mutual interaction. Importantly, the contribution of each factor could be modeled at different degrees of semantic and spatiotemporal congruency, and not simply in the more classical dichotomous fashion (e.g., synchronous vs asynchronous). Samad and colleagues independently proposed a similar Bayesian inference model for the classic RHI, including visual, proprioceptive and tactile components (Samad et al., 2015). They further implemented the model and compared its predictions with data collected from ad-hoc performed experiments, showing a good agreement among the two. Noticeably, they showed how this class of inference models is able to predict that ownership illusion can occur also based on visuoproprioceptive information alone, corroborating in this way our earlier result.

An alternative computational framework proposed for the study of self-processing, including the sense of body ownership, is predicting coding (Apps and Tsakiris, 2013; Seth, 2013). In general predictive coding accounts are based on the Helmoholtzian view that the brain needs to infer the hidden causes of the sensory signals, and that it does so by minimizing the error between predictions from its internal causal model and the available sensory information (Rao and Ballard,1999; Srinivasan et al., 1982). Applied to the case of the RHI, predictive coding proposals hold that the illusion results from minimizing the prediction error arising from seeing the touch on the rubber hand and feeling it on the real hand.To minimize the error the brain could adopt several "strategies", for example recoding the position of the real hand towards the location of the seen touch and updating its prior beliefs about the one's own hand appearance (Apps and Tsakiris, 2013), and/or by attenuating the somatosensory processing from the real hand (Zeller et al., 2014). Although these proposals provided interesting insights and can potentially account for several observed correlates of ownership illusions, their actual implementation is still missing. Bayesian inference models similar to our proposal, are easier to implement. In fact, as we proposed, implementations like the one presented in Samad et al. (2015), could be extended to include other factors (e.g., semantic and visuomotor information), and further interfaced with neural network models able to simulate processes like the multisensory integration 
of visuotactile stimuli on the hand (Magosso, 2010; Magosso et al., 2010).

\section{OWNERSHIP ILLUSIONS RELAXES TEMPORAL CONSTRAINTS FOR VISUOTACTILE INTEGRATION}

The study presented in Chapter 2 brought, among the other results discussed above, a serendipitous finding. When the ownership illusion was established, in our case through visuoproprioceptive coupling, asynchronous visuotactile stimuli were not perceived as wrong, which was instead the case when the illusion was not experienced. Building up on this result, in the study presented in Chapter 3, we designed an experiment to formally test the hypothesis that, when undergoing an ownership illusion, the temporal constraints for visuotactile integration are relaxed as a result of the established causal binding between the real and the fake body.The study provided a robust support in favor of this hypothesis. These results provided the first evidence for the impact of ongoing body ownership illusions on the processing of multisensory information, and fit consistently with several effects highlighted by previous research on multimodal integration.

It was previously shown that vision of the hand facilitates visuotactile integration by extending the temporal window in which two independent, tactile (vibration on the hand) and visual (flashing led) stimuli (Ide and Hidaka, 2013). This effect was found only if the hand image was shown in an anatomically plausible position, indicating that the effect was linked to some aspect of self-body processing. Nevertheless, differently from what we did, that study did not address any effect of ownership, as the design consisted in just showing a drawn hand image on a screen placed in front on the participants, far in space from the hand receiving the touch.

Interestingly, a more recent study reported complementary findings that apparently contradict our results. Keys and colleagues showed that modulating the sense of ownership towards a rubber hand, by controlling the plausibility of its anatomical posture, do not affect the detection of visuotactile asynchrony (Keys et al., 2018). A substantial difference with respect to our study that may explain the different outcome is rooted in the causal relationship between the visual and tactile stimuli to be integrated. In testing multisensory interaction, Keys and colleagues adopted the classical choice of a flashing LED presented next to the finger receiving the tactile stimuli. We instead adopted a visual stimulus semantically congruent with the delivered touch, namely the brief rotation of a geared wheel seen in touch with the virtual finger corresponding to the one receiving the touch. This is a crucial difference because while a LED flash, however close to the body, cannot generate by itself a mechanical vibration resulting in a tactile sensation, this is the case for the case of a geared wheel sliding on a finger. It is the causal binding established during ownership illusions, between events seen on the virtual body and semantically congruent somatosensory stimuli on the real one, that does relax constraints for multisensory integration, not the illusion itself.

In support of our view, previous research reported consistent evidence for how the perceived temporal relationship between stimuli and/or events is flexible and dependent on their implicit causal association. This is the case of the intentional-binding effect, in which a willed action and its associated sensory feedback are perceived closer in time than they actually are (Haggard et al., 2002; Moore and Obhi, 2012). Expansions of the temporal window of integration similar 
to the one we found, have been attributed to the causal binding of audiovisual stimuli in speech perception (Vatakis et al., 2008; Vatakis and Spence, 2007). Another effect in line with our finding is the simultaneity constancy effect. In this case, flexible temporal windows for the integration of multisensory stimuli associated to the same events are considered as a compensatory mechanism for the intrinsic differences in the transduction and/or transmission times of the different sensory channels, and for the fact that such differences are modulated by environmental conditions like illumination and temperature (Harrar and Harris, 2005; Kopinska and Harris, 2004). Cast within this wider framework, our results indicate that the sense of body ownership involves predictive cause-effects mechanisms that shape the processing of bodily signals during interactions with the environment.

\section{THE SENSE OF OWNERSHIP OVER A SEMI-TRANSPARENT BODY INCREASES PAIN SENSITIVITY}

The results presented in Chapter 4 provide novel insights into how, during ownership illusions, the appearance of the surrogate body modulate pain perception. In particular, we showed that when experiencing an ownership illusion over a semi-transparent body, higher levels of experienced ownership resulted in an increased pain sensitivity. Previous studies have shown that the analgesic effect of having direct view of one's own body while receiving painful stimuli (Longo et al., 2009), holds true when looking at a collocated virtual body during ownership illusions (Martini et al., 2014; Nierula et al., 2017). Considering this evidence, our finding could seem to contradict the naïve expectation that analgesic effects would fade away together with the vision of the body and with the associated diminished sense of ownership. Nevertheless, to a closer inspection our intriguing findings could be accounted by at least two alternative explanations. They may reflect a top-down modulation of pain in which body transparency is interpreted as a form of weakness and increased vulnerability. This is in line with independent evidences for the increased pain sensitivity associated with the view of a negatively-valued body, e.g., injured, reddened, or swollen (Martini et al., 2013; Osumi et al., 2014b, 2014a). Alternatively, the trend we found could be related to the interference of body semi-transparency with lower level mechanisms underlying pain modulation. Pain perception was in fact linked with the intracortical excitability in the primary somatosensory cortex (Longo et al., 2012).Accordingly, the impact of body vision on pain sensitivity was associated with an increased intracortical inhibition within the somatosensory cortex, and with a reorganization of somatosensory maps consisting in sharpened neurones receptive fields, both inhibiting the perception of painful stimuli (Haggard et al., 2013).Along these lines, we speculated that when the "owned" body appears to be semi-transparent, these mechanisms of sharpening and reorganization may be not as effective. Nevertheless, as we did not foreseen the actual outcome of our study, our design was not optimal to formally test these speculative interpretations. Despite this, having shown that the apparent level of body transparency does modulate pain sensitivity, our work paved the way to future studies that could investigate potential clinical application of IVR protocols for pain treatment.

\section{THE SENSE OF OWNERSHIP AND SELF-LOCATION ARE SELECTIVELY DISSOCIABLE IN BODILY ILLUSIONS}

Self-location and body ownership are considered to be two fundamental components of selfconsciousness (Blanke and Metzinger, 2009). However, the extent to which these two aspects can be selectively altered during full-body illusions has been, and still is, an important matter of debate. 
Numerous studies consistently reported that ownership towards a filmed or a virtual body is experienced together with changes in self-location during illusory out-of-body experiences (OBEs) induced with the back-stroking paradigm (Blanke, 2012; lonta et al., 2011; Lenggenhager, 2007; Serino et al., 2013). On the other hand, an independent brunch of studies consistently reported that in order to experience ownership towards a surrogate body a first person perspective over the body is a must (Maselli and Slater, 2013; Petkova et al., 2011; Petkova and Ehrsson, 2008). How to reconcile these two opposite lines of evidence? In our previous study (see discussion in Chapter 2), we proposed that a possible explanation to the puzzle is linked to the fact that what in OBEs experiments is reported to be ownership is rather a form self-identification similar to the case of recognizing oneself in a mirror (Maselli and Slater, 2013). In our view, the main difference between ownership and self-identification is that the latter lacks the key visuoproprioceptive coupling that characterizes illusory ownership. In fact, the reports of ownership from OBEs studies are exclusively based on questionnaire data and self-reports in debriefing sessions, assessments that cannot test this difference. The study presented in Chapter 6, tested the hypothesis that manipulations of self-location and body ownership in FBOls and OBEs rely on different and dissociable, although interacting, multisensory processes. The study was designed ad-hoc for testing the following hypothesis. While changes in the sense of ownership are rooted in the visuoproprioceptice coupling of visual stimuli from the surrogate body and somatosensory stimuli from the real body as we previously suggested (Maselli and Slater, 2013), changes in the perceived self-location are rooted in the expansion of the visual receptive fields of visuotactile neuronal populations. Our results clearly demonstrated the validity of this hypothesis with objective measures from behavioural postural tasks and psychophysical tests (Maselli and Slater, 2014).

To our knowledge our study it still now the only experimental work that simultaneously tested how both the sense of ownership and self-location are altered during full bodily illusions with different conditions of visual perspective over the fake body. Additional studies with a similar approach are needed to corroborate and extend our results. In fact, numerous studies still overlook the important point of disentangling genuine alterations of the sense of ownership from actual changes in the sense of self-location during full body illusions triggered from a third person perspective. In particular, neuroimaging studies are needed to shed more light into the different brain processes associated with the different patterns of alterations in the sense of ownership and self-location corresponding to different types of bodily illusion. In fact, while there is a rich literature of imaging studies focusing selectively in one of the two components of self-consciousness (see Section 1.2.3 for references), a deeper and more comprehensive look on how these the two components interact and/or can be dissociated is still missing.

\section{Allocentric and egocentric manipulation of SElf-Location IN ILLUSORY OBEs} In the thorough analysis of the relevant literature carried out to inspect the differences that tease a part alterations of ownership and self-location (Maselli and Slater, 2014), we further emphasized important aspects that differentiate illusory OBEs triggered via the back-stroking paradigm from front-stroking induced OBEs. In both, a third-person-perspective over a distant body seen from behind is given and the illusion is triggered by synchronous visuotactile stimulation. However, in the front-stroking case, seen and felt touch are spatially coincident in the disembodied empty space, 
as seen through the visor, where the real body is located but occluded (Ehrsson, 2007). In the backstroking case seen and felt touch are instead in spatial conflict (Lenggenhager, 2007). These different experimental paradigms correspond to important differences in the resulting illusory experience.As largely discussed in Chapters 1 and 7, far from being subtle, such differences encompass all aspects of the corresponding illusory experience, from subjective reports, to behavioral and neuronal correlates. Despite this, in the literature there is to date an utter lack of discussion and inquire about the issue.

In Chapter 7 a detailed overview of the key differences characterizing illusory changes in self-location induced by back-stroking and front-stroking OBEs is presented along with a thoughtful analysis of the possible implications for a comprehensive understanding of the sense of self-location as a constitutive component of self-consciousness. As a new proposal, it is suggested that the sense of self-location may be regarded as the blending of two parallel representations. An abstract allocentric coding of the position occupied in the environment, and the egocentric mapping of somatosensory sensations into the external space.Although future experimental work is needed to corroborate this speculative view, this work represents an important example of the effort for comparing in a comprehensive and constructive approach different brunches of experimental research. To pursue crucial advances in our current understanding of the complex brain machinery underlying self-perception, it is desirable that this type of approach will spread out, inspiring further novel experimental practices. 


\section{References}

Apps, M. a J., and Tsakiris, M. (2013). The free-energy self: Brain Res. 228, 43-50.

A predictive coding account of self-recognition. Neurosci.

Biobehav. Rev.

lonta, S., Heydrich, L., Lenggenhager, B., Mouthon, M., Fornari, E., Chapuis, D., et al. (2011). Multisensory mechanisms

Blanke, O. (2012). Multisensory brain mechanisms of bodily in temporo-parietal cortex support self-location and first-person self-consciousness. Nat. Rev. Neurosci. 13, 556-71. perspective. Neuron 70, 363-74.

Blanke, O., and Metzinger, T. (2009). Full-body illusions and Keys, R. T., Rich, A. N., and Zopf, R. (2018). Multisensory minimal phenomenal selfhood. Trends Cogn. Sci. 13, 7-13. temporal processing in own-body contexts: plausibility of hand ownership does not improve visuo-tactile asynchrony detection.

Botvinick, M. (2004). Probing the Neural Basis of Body Exp. Brain Res. 236,1-13.

Ownership. Science 305, 782-783.

Kopinska,A., and Harris, L. R. (2004). Simultaneity constancy. Botvinick, M., and Cohen, J. (1998). Rubber hands "feel” touch Perception 33, 1049-1060.

that eyes see. Nature 391, 756.

Lenggenhager, B. (2007). Video Ergo Sum: Manipulating Ehrsson, H. H. (2007). The experimental induction of out-of- Bodily Self-Consciousness. Science (80-. ). 1096, 1095-1099. body experiences. Science (80-. ). 317, 1048.

Longo, M. R., Betti, V., Aglioti, S. M., and Haggard, P. (2009). Ehrsson, H. H., Spence, C., and Passingham, R. E. (2004). Visually induced analgesia: seeing the body reduces pain. J. That's my hand! Activity in premotor cortex reflects feeling of Neurosci. 29, 12125-30.

ownership of a limb. Science 305, 875-877.

Longo, M. R., lannetti, G. D., Mancini, F., Driver, J., and González-Franco, M., Pérez-marcos, D., Spanlang, B., Haggard,P.(2012). Linking pain and the body: neural correlates and Slater, M. (2010). The Contribution of Real-Time Mirror of visually induced analgesia. J. Neurosci. 32, 2601-7.

Reflections of Motor Actions on Virtual Body Ownership in an

Immersive Virtual Environment. IEEE Virtual Real., 111-114. Magosso, E. (2010). Integrating information from vision and touch: a neural network modeling study. IEEETrans. Inf.Technol. Graziano, M. S. A., and Botvinick, M. (2002). “How the Biomed.14, 598-612. doi:10.1109/TITB.2010.2040750. brain represents the body: insights from neurophysiology and psychology", in Common Mechanisms in Perception and Magosso, E., Ursino, M., di Pellegrino, G., Làdavas, E., and Action:Attention and Performance, ed.W. Prinz and B. Serino, A. (2010). Neural bases of peri-hand space plasticity through tool-use: insights from a combined computationalHommel (Oxford University Press, Oxford England), 136- experimental approach. Neuropsychologia 48, 812-30. 157.

Makin, T. R., Holmes, N. P., and Ehrsson, H. H. (2008). On Haggard, P., Clark, S., and Kalogeras, J. (2002). Voluntary the other hand: dummy hands and peripersonal space. Behav. action and conscious awareness. Nat. Neurosci. 5, 382-385. Brain Res. 191, 1-10

Haggard, P., lannetti, G. D., and Longo, M. R. (2013). Spatial Martini, M., Kilteni, K., Maselli, A., and Sanchez-vives, M. sensory organization and body representation in pain perception. $V$ (2015). The body fades away: investigating the effects of Curr. Biol. 23, R164-R176. transparency of an embodied virtual body on pain threshold and body ownership. Sci. Rep. 5, 13948.

Harrar, V., and Harris, L. R. (2005). Simultaneity constancy: detecting events with touch and vision. Exp. brain Res. 166, Martini, M., Perez-Marcos, D., and Sanchez-Vives, M. V. 465-73

(2013). What Color is My Arm? Changes in Skin Color of an Embodied Virtual Arm Modulates Pain Threshold. Front. Hum.

Ide, M., and Hidaka, S. (2013). Visual presentation of hand Neurosci. 7, 438.

image modulates visuo-tactile temporal order judgment. Exp. 
Martini, M., Perez-Marcos, D., and Sanchez-Vives, M. V. Jimenez, A., and Lopez, C. (2013). Bodily ownership and self(2014). Modulation of pain threshold by virtual body ownership. location: Components of bodily self-consciousness. Conscious. Eur. J. Pain (United Kingdom) 18, 1040-1048. Cogn. 22, 1239-1252.

Maselli,A., and Slater, M. (2013). The building blocks of the full Seth, A. K. (2013). Interoceptive inference, emotion, and the body ownership illusion. Front. Hum. Neurosci. 7, 83. embodied self.Trends Cogn. Sci., 1-9.

Maselli, A., and Slater, M. (2014). Sliding perspectives: Slater, M., Spanlang, B., Sanchez-Vives, M. V, and Blanke, dissociating ownership from self-location during full body O. (2010). First person experience of body transfer in virtual illusions in virtual reality. Front. Hum. Neurosci. 8, 693. reality. PLoS One 5, e10564.

Moore, J.W., and Obhi, S. S. (2012). Intentional binding and Srinivasan, M.V, Laughlin, S. B., and Dubs,A. (1982). Predictive the sense of agency:A review. Conscious. Cogn. 21, 546-561 coding: a fresh view of inhibition in the retina. Proc. R. Soc. Lond. B 216, 427-259.

Nierula, B., Martini, M., Matamala-Gomez, M.,Slater, M., and Sanchez-Vives, M.V. (2017). Seeing an Embodied Virtual Hand Tieri, G., Tidoni, E., Pavone, E. F., and Aglioti, S. M. (2015). is Analgesic Contingent on Colocation. J. Pain 18, 645-655.

Mere observation of body discontinuity affects perceived ownership and vicarious agency over a virtual hand. Exp. Brain

Osumi, M., Imai, R., Ueta, K., Nakano, H., Nobusako, S., and Res. 233, 1247-1259.

Morioka, S. (2014a). Factors associated with the modulation of pain by visual distortion of body size. Front. Hum. Neurosci. Vatakis,A., Ghazanfar,A. a, and Spence, C. (2008). Facilitation $8,137$. of multisensory integration by the "unity effect" reveals that speech is special. J.Vis. 8,14 .

Osumi, M., Imai, R., Ueta, K., Nobusako, S., and Morioka, S. (2014b). Negative body image associated with changes in the Vatakis, A., and Spence, C. (2007). Crossmodal binding: visual body appearance increases pain perception. PLoS One evaluating the "unity assumption" using audiovisual speech 9 , e107376. stimuli. Percept. Psychophys. 69, 744-756

Petkova, V. I., and Ehrsson, H. H. (2008). If I were you: Zeller, D., Litvak, V., Friston, K. J., and Classen, J. (2014). perceptual illusion of body swapping. PLoS One 3, e3832. Sensory Processing and the Rubber Hand Illusion- An Evoked

Petkova, V. I., Khoshnevis, M., and Ehrsson, H. H. (2011). Potential Study. J. Cogn. Neurosci.

The perspective matters! Multisensory integration in egocentric reference frames determines full-body ownership. Front. Psychol. 2, 35.

Rao, R. P., and Ballard, D. H. (1999). Predictive coding in the visual cortex: a functional interpretation of some extra-classical receptive-field effects. Nat. Neurosci. 2, 79-87.

Samad, M., Chung, A. J., and Shams, L. (2015). Perception of Body Ownership Is Driven by Bayesian Sensory Inference. PLoS One 10, e0117178

Sanchez-Vives, M. V, Spanlang, B., Frisoli, A., Bergamasco, M., and Slater, M. (2010). Virtual hand illusion induced by visuomotor correlations. PLoS One 5, e10381.

Serino,A.,Alsmith,A., Costantini, M., Mandrigin,A.,Tajadura- 



\section{ACKNOWLEDGMENTS}

Foremost, I would like to express my great appreciation and gratitude to my supervisor Prof. Mel Slater for giving me the unique opportunity to undertake a new path in my life. Mel offered me the chance to join his vibrant lab, when I was searching for the big change - leave my career path in Astrophysics and follow a growing and compelling interest in human perception and cognition. I will always be grateful for his intrepid choice. Likewise, I thank Mel for introducing and guiding me through the intriguing Science of Virtual Reality and of its application to Psychology and Neuroscience. His vision and knowledge has been a unique lesson.

I am extremely grateful to Prof. Beatrice de Gelder for supporting with enthusiasm my candidature as an external PhD at Maastricht University and for helping me in the process. I sincerely hope that this will pave the way for future engaging collaborations.

I would like to thank all members of the Event Lab with whom I have shared an exciting period of my academic life and from whom I have learned so much. A special thank goes to my close friends and colleagues Konstantina, Sofia, Irene, Mar and Marta, all of whom inspired diverse factes of my work and life.

I thank my dear friend Laura for helping me in putting all this scientific work in nicely shaped layout. Last but not least, my love and gratitude goes to Ivano for his unconditional support. 
